\title{
Electronic cigarettes for smoking cessation (Review)
}

Hartmann-Boyce J, McRobbie H, Lindson N, Bullen C, Begh R, Theodoulou A, Notley C, Rigotti NA, Turner T, Butler AR, Fanshawe TR, Hajek P

Hartmann-Boyce J, McRobbie H, Lindson N, Bullen C, Begh R, Theodoulou A, Notley C, Rigotti NA, Turner T, Butler AR, Fanshawe TR, Hajek P.

Electronic cigarettes for smoking cessation.

Cochrane Database of Systematic Reviews 2021, Issue 4. Art. No.: CD010216.

DOI: 10.1002/14651858.CD010216.pub5.

www.cochranelibrary.com 
TABLE OF CONTENTS

HEADER

ABSTRACT

PLAIN LANGUAGE SUMMARY

SUMMARY OF FINDINGS

BACKGROUND

OBJECTIVES

METHODS

RESULTS

Figure 1.

Figure 2.

Figure 3.

Figure 4.

Figure 5.

DISCUSSION

AUTHORS' CONCLUSIONS

ACKNOWLEDGEMENTS

REFERENCES

CHARACTERISTICS OF STUDIES

DATA AND ANALYSES

Analysis 1.1. Comparison 1: Nicotine EC versus NRT, Outcome 1: Smoking cessation

Analysis 1.2. Comparison 1: Nicotine EC versus NRT, Outcome 2: Adverse events

Analysis 1.3. Comparison 1: Nicotine EC versus NRT, Outcome 3: Serious adverse events

Analysis 1.4. Comparison 1: Nicotine EC versus NRT, Outcome 4: Carbon monoxide (ppm)

Analysis 1.5. Comparison 1: Nicotine EC versus NRT, Outcome 5: Heart rate (bpm)

Analysis 1.6. Comparison 1: Nicotine EC versus NRT, Outcome 6: Systolic blood pressure

Analysis 1.7. Comparison 1: Nicotine EC versus NRT, Outcome 7: Blood oxygen saturation

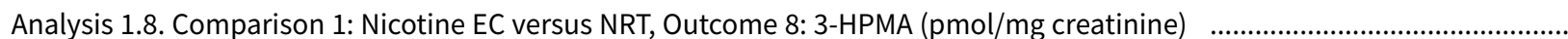

Analysis 1.9. Comparison 1: Nicotine EC versus NRT, Outcome 9: NNAL (pmol/mg creatinine))

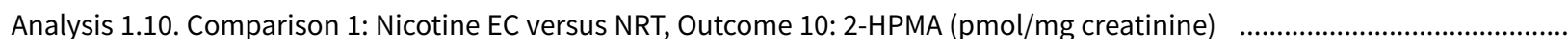

Analysis 1.11. Comparison 1: Nicotine EC versus NRT, Outcome 11: HMPMA (pmol/mg creatinine)

Analysis 1.12. Comparison 1: Nicotine EC versus NRT, Outcome 12: PheT (pmol/mg creatinine)

Analysis 1.13. Comparison 1: Nicotine EC versus NRT, Outcome 13: CEMA (pmol/mg creatinine)

Analysis 1.14. Comparison 1: Nicotine EC versus NRT, Outcome 14: AAMA (pmol/mg creatinine)

Analysis 1.15. Comparison 1: Nicotine EC versus NRT, Outcome 15: FEV1 (ml)

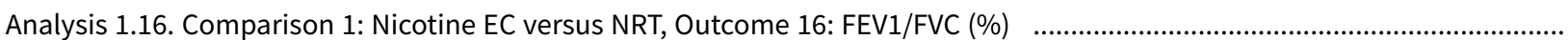

Analysis 2.1. Comparison 2: Nicotine EC versus varenicline, Outcome 1: Smoking cessation

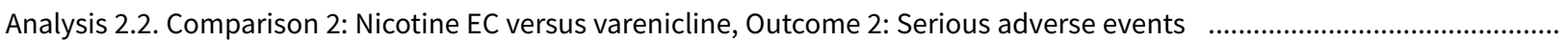

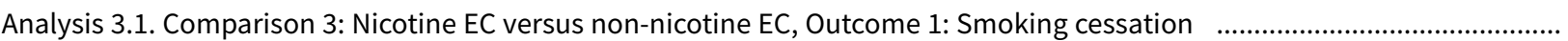

Analysis 3.2. Comparison 3: Nicotine EC versus non-nicotine EC, Outcome 2: Adverse events

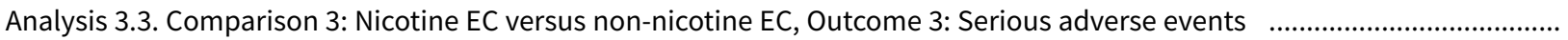

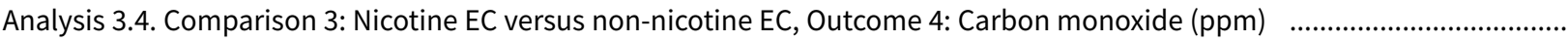

Analysis 3.5. Comparison 3: Nicotine EC versus non-nicotine EC, Outcome 5: Heart rate

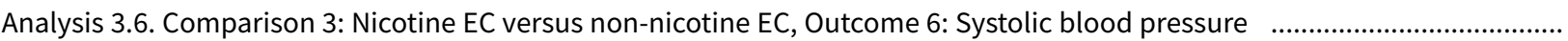

Analysis 3.7. Comparison 3: Nicotine EC versus non-nicotine EC, Outcome 7: FeNO (ppb)

Analysis 3.8. Comparison 3: Nicotine EC versus non-nicotine EC, Outcome 8: FEV1 (l)

Analysis 3 9. Comparison 3. Nicotine EC versus non-nicotine EC, Outcome 9: FVC (I)

Analysis 3.10. Comparison 3: Nicotine EC versus non-nicotine EC, Outcome 10: FEV1/FVC

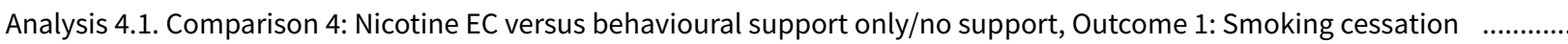

Analysis 4.2. Comparison 4: Nicotine EC versus behavioural support only/no support, Outcome 2: Adverse events ..................

Analysis 4.3. Comparison 4: Nicotine EC versus behavioural support only/no support, Outcome 3: Serious adverse events .....

Analysis 4.4. Comparison 4: Nicotine EC versus behavioural support only/no support, Outcome 4: Carbon monoxide (ppm) ... 
Analysis 4.5. Comparison 4: Nicotine EC versus behavioural support only/no support, Outcome 5: Heart rate (bpm) ............... Analysis 4.6. Comparison 4: Nicotine EC versus behavioural support only/no support, Outcome 6: Systolic blood pressure ..... Analysis 4.7. Comparison 4: Nicotine EC versus behavioural support only/no support, Outcome 7: Blood oxygen saturation ... Analysis 4.8. Comparison 4: Nicotine EC versus behavioural support only/no support, Outcome 8: 3-HPMA (SMD) .................. Analysis 4.9. Comparison 4: Nicotine EC versus behavioural support only/no support, Outcome 9: NNAL (SMD) ...................... Analysis 4.10. Comparison 4: Nicotine EC versus behavioural support only/no support, Outcome 10: 2-HPMA (pmol/mg creatinine)

Analysis 4.11. Comparison 4: Nicotine EC versus behavioural support only/no support, Outcome 11: HMPMA (pmol/mg creatinine)

Analysis 4.12. Comparison 4: Nicotine EC versus behavioural support only/no support, Outcome 12: PheT (pmol/mg creatinine)

Analysis 4.13. Comparison 4: Nicotine EC versus behavioural support only/no support, Outcome 13: CEMA (pmol/mg creatinine)

Analysis 4.14. Comparison 4: Nicotine EC versus behavioural support only/no support, Outcome 14: AAMA (pmol/mg creatinine)

Analysis 4.15. Comparison 4: Nicotine EC versus behavioural support only/no support, Outcome 15: S-PMA (nanograms) ...... Analysis 4.16. Comparison 4: Nicotine EC versus behavioural support only/no support, Outcome 16: FVC (litres) ................... Analysis 4.17. Comparison 4: Nicotine EC versus behavioural support only/no support, Outcome 17: FEV1 (litres) ................... Analysis 4.18. Comparison 4: Nicotine EC versus behavioural support only/no support, Outcome 18: FEF 25-75 (litres/second)) Analysis 4.19. Comparison 4: Nicotine EC versus behavioural support only/no support, Outcome 19: PEF 25-75 (litres/minute) . Analysis 5.1. Comparison 5: Nicotine EC + NRT versus non-nicotine EC + NRT, Outcome 1: Smoking cessation

Analysis 5.2. Comparison 5: Nicotine EC + NRT versus non-nicotine EC + NRT, Outcome 2: Adverse events

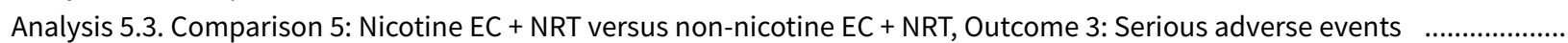
Analysis 5.4. Comparison 5: Nicotine EC + NRT versus non-nicotine EC + NRT, Outcome 4: Carbon monoxide (ppm) ................ Analysis 5.5. Comparison 5: Nicotine EC + NRT versus non-nicotine EC + NRT, Outcome 5: FeNO (ppb)

Analysis 5.6. Comparison 5: Nicotine EC + NRT versus non-nicotine EC + NRT, Outcome 6: FEV1 (\%)

Analysis 5.7. Comparison 5: Nicotine EC + NRT versus non-nicotine EC + NRT, Outcome 7: FVC (\%)

Analysis 6.1. Comparison 6: Nicotine EC + NRT versus NRT, Outcome 1: Smoking cessation

Analysis 6.2. Comparison 6: Nicotine EC + NRT versus NRT, Outcome 2: Adverse events

Analysis 6.3. Comparison 6: Nicotine EC + NRT versus NRT, Outcome 3: Serious adverse events

Analysis 7.1. Comparison 7: Higher versus lower nicotine content, Outcome 1: Serious adverse events

Analysis 7.2. Comparison 7: Higher versus lower nicotine content, Outcome 2: Carbon monoxide (ppm)

Analysis 7.3. Comparison 7: Higher versus lower nicotine content, Outcome 3: Heart rate

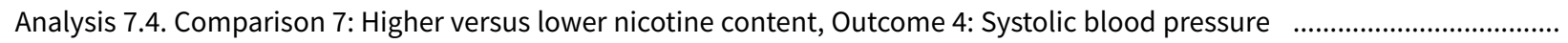

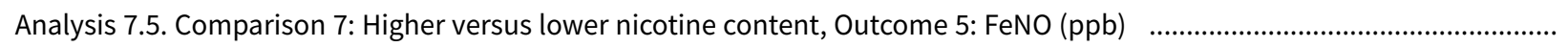

Analysis 7.6. Comparison 7: Higher versus lower nicotine content, Outcome 6: FEV1 (l)

Analysis 7.7. Comparison 7: Higher versus lower nicotine content, Outcome 7: FVC (I)

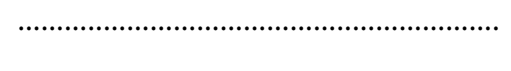

Analysis 7.8. Comparison 7: Higher versus lower nicotine content, Outcome 8: FEV1/FVC

Analysis 8.1. Comparison 8: Non-nicotine EC versus behavioural support only/no support, Outcome 1: Smoking cessation ....

Analysis 8.2. Comparison 8: Non-nicotine EC versus behavioural support only/no support, Outcome 2: Adverse events at 12 weeks

Analysis 8.3. Comparison 8: Non-nicotine EC versus behavioural support only/no support, Outcome 3: Serious adverse events at 24 weeks

Analysis 9.1. Comparison 9: Non-nicotine EC + NRT versus NRT, Outcome 1: Smoking cessation

Analysis 9.2. Comparison 9: Non-nicotine EC + NRT versus NRT, Outcome 2: Adverse events

Analysis 9.3. Comparison 9: Non-nicotine EC + NRT versus NRT, Outcome 3: Serious adverse events ....

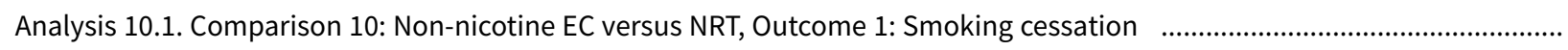

Analysis 10.2. Comparison 10: Non-nicotine EC versus NRT, Outcome 2: Adverse events

Analysis 10.3. Comparison 10: Non-nicotine EC versus NRT, Outcome 3: Serious adverse events 
[Intervention Review]

\section{Electronic cigarettes for smoking cessation}

Jamie Hartmann-Boyce ${ }^{1}$, Hayden McRobbie ${ }^{2}$, Nicola Lindson ${ }^{1}$, Chris Bullen ${ }^{3}$, Rachna Begh 1 , Annika Theodoulou ${ }^{1}$, Caitlin Notley ${ }^{4}$ Nancy A Rigotti5, Tari Turner6, Ailsa R Butler1, Thomas R Fanshawe1, Peter Hajek7

1Nuffield Department of Primary Care Health Sciences, University of Oxford, Oxford, UK. ${ }^{2}$ National Drug and Alcohol Research Centre, University of New South Wales, Sydney, Australia. ${ }^{3}$ National Institute for Health Innovation, University of Auckland, Auckland, New Zealand. ${ }^{4}$ Norwich Medical School, University of East Anglia, Norwich, UK. 5 Tobacco Research and Treatment Center, Department of Medicine, Massachusetts General Hospital and Harvard Medical School, Boston, Massachusetts, USA. ${ }^{6}$ Cochrane Australia, School of Public Health \& Preventive Medicine, Monash University, Melbourne, Australia. ${ }^{7}$ Wolfson Institute of Preventive Medicine, Barts \& The London School of Medicine and Dentistry, Queen Mary University of London, London, UK

Contact address: Jamie Hartmann-Boyce, jamie.hartmann-boyce@phc.ox.ac.uk.

Editorial group: Cochrane Tobacco Addiction Group.

Publication status and date: New search for studies and content updated (conclusions changed), published in Issue 4, 2021.

Citation: Hartmann-Boyce J, McRobbie H, Lindson N, Bullen C, Begh R, Theodoulou A, Notley C, Rigotti NA, Turner T, Butler AR, Fanshawe TR, Hajek P. Electronic cigarettes for smoking cessation. Cochrane Database of Systematic Reviews 2021, Issue 4. Art. No.: CD010216. DOI: 10.1002/14651858.CD010216.pub5.

Copyright ( 2021 The Cochrane Collaboration. Published by John Wiley \& Sons, Ltd.

\section{A B S T R A C T}

\section{Background}

Electronic cigarettes (ECS) are handheld electronic vaping devices which produce an aerosol formed by heating an e-liquid. Some people who smoke use ECs to stop or reduce smoking, but some organizations, advocacy groups and policymakers have discouraged this, citing lack of evidence of efficacy and safety. People who smoke, healthcare providers and regulators want to know if ECs can help people quit and if they are safe to use for this purpose. This is an update of a review first published in 2014.

\section{Objectives}

To examine the effectiveness, tolerability, and safety of using electronic cigarettes (ECs) to help people who smoke achieve long-term smoking abstinence.

\section{Search methods}

We searched the Cochrane Tobacco Addiction Group's Specialized Register, the Cochrane Central Register of Controlled Trials (CENTRAL), MEDLINE, Embase, and PsycINFO to 1 February 2021, together with reference-checking and contact with study authors.

\section{Selection criteria}

We included randomized controlled trials (RCTs) and randomized cross-over trials in which people who smoke were randomized to an $\mathrm{EC}$ or control condition. We also included uncontrolled intervention studies in which all participants received an EC intervention. To be included, studies had to report abstinence from cigarettes at six months or longer and/or data on adverse events (AEs) or other markers of safety at one week or longer.

\section{Data collection and analysis}

We followed standard Cochrane methods for screening and data extraction. Our primary outcome measures were abstinence from smoking after at least six months follow-up, adverse events (AEs), and serious adverse events (SAEs). Secondary outcomes included changes in carbon monoxide, blood pressure, heart rate, blood oxygen saturation, lung function, and levels of known carcinogens/toxicants. We used a fixed-effect Mantel-Haenszel model to calculate the risk ratio (RR) with a 95\% confidence interval (CI) for dichotomous outcomes. For continuous outcomes, we calculated mean differences. Where appropriate, we pooled data from these studies in meta-analyses. 


\section{Main results}

We included 56 completed studies, representing 12,804 participants, of which 29 were RCTs. Six of the 56 included studies were new to this review update. Of the included studies, we rated five (all contributing to our main comparisons) at low risk of bias overall, 41 at high risk overall (including the 25 non-randomized studies), and the remainder at unclear risk.

There was moderate-certainty evidence, limited by imprecision, that quit rates were higher in people randomized to nicotine EC than in those randomized to nicotine replacement therapy (NRT) (risk ratio (RR) $1.69,95 \%$ confidence interval $(\mathrm{Cl}) 1.25$ to $2.27 ; \mathrm{I}^{2}=0 \% ; 3$ studies, 1498 participants). In absolute terms, this might translate to an additional four successful quitters per $100(95 \% \mathrm{Cl} 2$ to 8$)$. There was lowcertainty evidence (limited by very serious imprecision) that the rate of occurrence of AEs was similar) $(\mathrm{RR} 0.98,95 \% \mathrm{Cl} 0.80$ to $1.19 ; 12=0 \%$; 2 studies, 485 participants). SAEs occurred rarely, with no evidence that their frequency differed between nicotine EC and NRT, but very serious imprecision led to low certainty in this finding ( $R R 1.37,95 \% \mathrm{Cl} 0.77$ to $2.41: \mathrm{I}^{2}=\mathrm{n} / \mathrm{a} ; 2$ studies, 727 participants).

There was moderate-certainty evidence, again limited by imprecision, that quit rates were higher in people randomized to nicotine EC than to non-nicotine EC (RR 1.70, 95\% Cl 1.03 to 2.81; $\left.\right|^{2}=0 \%$; 4 studies, 1057 participants). In absolute terms, this might again lead to an additional four successful quitters per $100(95 \% \mathrm{Cl} 0$ to 11$)$. These trials mainly used older EC with relatively low nicotine delivery. There was moderate-certainty evidence of no difference in the rate of AEs between these groups (RR $1.01,95 \% \mathrm{Cl} 0.91$ to $1.11 ; \mathrm{I}^{2}=0 \%$; 3 studies, 601 participants). There was insufficient evidence to determine whether rates of SAEs differed between groups, due to very serious imprecision (RR $0.60,95 \% \mathrm{Cl} 0.15$ to $2.44 ; \mathrm{I}^{2}=\mathrm{n} / \mathrm{a} ; 4$ studies, 494 participants).

Compared to behavioral support only/no support, quit rates were higher for participants randomized to nicotine $\mathrm{EC}(\mathrm{RR} 2.70,95 \% \mathrm{Cl} 1.39$ to $5.26 ; \mathrm{I}^{2}=0 \%$; 5 studies, 2561 participants). In absolute terms this represents an increase of seven per 100 ( $95 \% \mathrm{Cl} 2$ to 17$)$. However, this finding was of very low certainty, due to issues with imprecision and risk of bias. There was no evidence that the rate of SAEs differed, but some evidence that non-serious AEs were more common in people randomized to nicotine EC (AEs: RR $1.22,95 \% \mathrm{Cl} 1.12$ to $1.32 ;\left.\right|^{2}=41 \%$, low certainty; 4 studies, 765 participants; SAEs: RR 1.17, 95\% CI 0.33 to 4.09; I2 = 5\%; 6 studies, 1011 participants, very low certainty).

Data from non-randomized studies were consistent with RCT data. The most commonly reported AEs were throat/mouth irritation, headache, cough, and nausea, which tended to dissipate with continued use. Very few studies reported data on other outcomes or comparisons and hence evidence for these is limited, with confidence intervals often encompassing clinically significant harm and benefit.

\section{Authors' conclusions}

There is moderate-certainty evidence that ECs with nicotine increase quit rates compared to ECs without nicotine and compared to NRT. Evidence comparing nicotine EC with usual care/no treatment also suggests benefit, but is less certain. More studies are needed to confirm the size of effect, particularly when using modern EC products. Confidence intervals were for the most part wide for data on AEs, SAEs and other safety markers, though evidence indicated no difference in AEs between nicotine and non-nicotine ECs. Overall incidence of SAEs was low across all study arms. We did not detect any clear evidence of harm from nicotine EC, but longest follow-up was two years and the overall number of studies was small.

The evidence is limited mainly by imprecision due to the small number of RCTs, often with low event rates. Further RCTs are underway. To ensure the review continues to provide up-to-date information, this review is now a living systematic review. We run searches monthly, with the review updated when relevant new evidence becomes available. Please refer to the Cochrane Database of Systematic Reviews for the review's current status.

\section{PLAIN LANGUAGE SUMMARY}

\section{Can electronic cigarettes help people stop smoking, and do they have any unwanted effects when used for this purpose?}

\section{What are electronic cigarettes?}

Electronic cigarettes (e-cigarettes) are handheld devices that work by heating a liquid that usually contains nicotine and flavorings. Ecigarettes allow you to inhale nicotine in a vapor rather than smoke. Because they do not burn tobacco, e-cigarettes do not expose users to the same levels of toxins that we know can cause smoking-related diseases in people who use conventional cigarettes.

Using an e-cigarette is known as 'vaping'. Many people use e-cigarettes to help them to stop smoking tobacco.

\section{Why we did this Cochrane Review}

Stopping smoking lowers your risk of getting lung cancer and other diseases. Many people find it difficult to quit. We wanted to find out if using e-cigarettes could help people to stop smoking, and if people using them for this purpose experienced any unwanted effects.

\section{What did we do?}

We searched for studies that looked at the use of e-cigarettes to help people stop smoking. 
We looked for randomized controlled trials, in which the treatments people received were decided at random. This type of study usually gives the most reliable evidence about the effects of a treatment. We also looked for studies in which everyone received an e-cigarette treatment.

We were interested in finding out:

- how many people stopped smoking for at least six months; and

- how many people had unwanted effects, reported on for at least one week.

Search date: We included evidence published up to 1st February 2021.

\section{What we found}

We found 56 studies in 12,804 adults who smoked. The studies compared e-cigarettes with:

- nicotine replacement therapy, such as patches or gum;

- varenicline (a medicine to help people stop smoking);

- nicotine-free e-cigarettes;

- behavioral support, such as advice or counseling; or

- no support, for stopping smoking.

Most studies took place in the USA (24 studies), the UK (9), and Italy (7).

\section{What are the results of our review?}

More people probably stop smoking for at least six months using nicotine e-cigarettes than using nicotine replacement therapy ( 3 studies, 1498 people), or nicotine-free e-cigarettes (4 studies, 1057 people).

Nicotine e-cigarettes may help more people to stop smoking than no support or behavioral support only (5 studies, 2561 people).

For every 100 people using nicotine e-cigarettes to stop smoking, 10 or 11 might successfully stop, compared with only six of 100 people using nicotine-replacement therapy or nicotine-free e-cigarettes, or four of 100 people having no support or behavioral support only.

We are uncertain if there is a difference between how many unwanted effects occur using nicotine e-cigarettes compared with nicotine replacement therapy, no support or behavioral support only. There was some evidence that non-serious unwanted effects were more common in groups receiving nicotine e-cigarettes compared to no support or behavioral support only. Similar low numbers of unwanted effects, including serious unwanted effects, were reported for other comparisons. There is probably no difference in how many non-serious unwanted effects occur in people using nicotine e-cigarettes compared to non-nicotine e-cigarettes.

The unwanted effects reported most often with nicotine e-cigarettes were throat or mouth irritation, headache, cough and feeling sick. These effects reduced over time as people continued using nicotine e-cigarettes.

\section{How reliable are these results?}

Our results are based on a small number of studies, and in some the measured data varied widely.

We are moderately confident that nicotine e-cigarettes help more people to stop smoking than nicotine replacement therapy or nicotinefree e-cigarettes. However, these results might change if further evidence becomes available.

We are less confident about how nicotine e-cigarettes compare with no support, or behavioral support, to stop smoking.

Most of our results for the unwanted effects are likely to change when more evidence becomes available.

\section{Key messages}

Nicotine e-cigarettes probably do help people to stop smoking for at least six months. They probably work better than nicotine replacement therapy and nicotine-free e-cigarettes.

They may work better than no support, or behavioral support alone, and they may not be associated with serious unwanted effects.

However, we need more, reliable evidence to be confident about the effects of e-cigarettes, particularly the effects of newer types of ecigarettes that have better nicotine delivery. 
S U M MARY OF FINDINGS

\section{Summary of findings 1 . Nicotine EC compared to NRT for smoking cessation}

\section{Nicotine EC compared to NRT for smoking cessation}

Patient or population: People who smoke

Setting: New Zealand, UK, USA

Intervention: Nicotine EC

Comparison: NRT

\begin{tabular}{|c|c|c|c|c|c|c|}
\hline \multirow[t]{2}{*}{ Outcomes } & \multicolumn{2}{|c|}{ Anticipated absolute effects ${ }^{*}(95 \% \mathrm{Cl})$} & \multirow{2}{*}{$\begin{array}{l}\text { Relative effect } \\
(95 \% \mathrm{Cl})\end{array}$} & \multirow{2}{*}{$\begin{array}{l}\text { № of partici- } \\
\text { pants } \\
\text { (studies) }\end{array}$} & \multirow{2}{*}{$\begin{array}{l}\text { Certainty of } \\
\text { the evidence } \\
\text { (GRADE) }\end{array}$} & \multirow[t]{2}{*}{ Comments } \\
\hline & Risk with NRT & Risk with Nicotine EC & & & & \\
\hline \multirow{2}{*}{$\begin{array}{l}\text { Smoking cessation at } 6 \text { months to } 1 \text { year } \\
\text { Assessed with biochemical validation }\end{array}$} & \multicolumn{2}{|l|}{ Study population } & \multirow{2}{*}{$\begin{array}{l}\text { RR } 1.69 \\
\text { (1.25 to } 2.27 \text { ) }\end{array}$} & \multirow{2}{*}{$\begin{array}{l}1498 \\
\text { (3 RCTs) }\end{array}$} & \multirow{2}{*}{$\begin{array}{l}\oplus \oplus \oplus \ominus \\
\text { MODERATE }\end{array}$} & \multirow[t]{2}{*}{-} \\
\hline & 6 per 100 & $\begin{array}{l}10 \text { per } 100 \\
(8 \text { to } 14)\end{array}$ & & & & \\
\hline \multirow{2}{*}{$\begin{array}{l}\text { Adverse events at } 4 \text { weeks to } 6 \text { months } \\
\text { Assessed by self-report }\end{array}$} & \multicolumn{2}{|l|}{ Study population } & \multirow{2}{*}{$\begin{array}{l}\text { RR } 0.98 \\
\text { (0.80 to 1.19) }\end{array}$} & \multirow{2}{*}{$\begin{array}{l}485 \\
\text { (2 RCTs) }\end{array}$} & \multirow{2}{*}{$\begin{array}{l}\oplus \oplus \odot \ominus \\
L O W b\end{array}$} & \multirow[t]{2}{*}{-} \\
\hline & 45 per 100 & $\begin{array}{l}44 \text { per } 100 \\
\text { (36 to } 53)\end{array}$ & & & & \\
\hline \multirow{2}{*}{$\begin{array}{l}\text { Serious adverse events at } 4 \text { weeks to } 1 \text { year } \\
\text { Assessed via self-report and medical records }\end{array}$} & \multicolumn{2}{|l|}{ Study population } & \multirow{2}{*}{$\begin{array}{l}\text { RR } 1.37 \\
\text { (0.77 to } 2.41 \text { ) }\end{array}$} & \multirow{2}{*}{$\begin{array}{l}727 \\
\text { (2 RCTs) }\end{array}$} & \multirow{2}{*}{$\begin{array}{l}\oplus \oplus \odot \odot \\
L O W b\end{array}$} & \multirow{2}{*}{$\begin{array}{l}\text { One study report- } \\
\text { ed no events; ef- } \\
\text { fect estimate based } \\
\text { on the one study in } \\
\text { which events were } \\
\text { reported }\end{array}$} \\
\hline & 5 per 100 & $\begin{array}{l}7 \text { per } 100 \\
(4 \text { to } 13)\end{array}$ & & & & \\
\hline
\end{tabular}

*The risk in the intervention group (and its $95 \%$ confidence interval) is based on the assumed risk in the comparison group and the relative effect of the intervention (and its $95 \% \mathrm{Cl}$ ). For cessation, the assumed risk in the control group is based on assumed quit rates for NRT assuming receipt of limited behavioral stop-smoking support (as per Hartmann-Boyce 2018a). The assumed risk for adverse events and serious adverse events is a weighted mean average of quit rates across control groups in contributing studies.

Cl: Confidence interval; RCT: randomized controlled trial; RR: Risk ratio

\section{GRADE Working Group grades of evidence}

High certainty: We are very confident that the true effect lies close to that of the estimate of the effect

Moderate certainty: We are moderately confident in the effect estimate: The true effect is likely to be close to the estimate of the effect, but there is a possibility that it is substantially different

Low certainty: Our confidence in the effect estimate is limited: The true effect may be substantially different from the estimate of the effect 
aDowngraded one level due to imprecision; small number of events ( $<300$ overall).

bDowngraded two levels due to imprecision; confidence intervals encompass clinically-important harm as well as clinically important benefit.

\section{Summary of findings 2 . Nicotine EC compared to non-nicotine EC for smoking cessation}

Nicotine EC compared to non-nicotine EC for smoking cessation

Patient or population: People who smoke cigarettes

Setting: Canada, Italy, New Zealand, UK, USA

Intervention: Nicotine EC

Comparison: Non-nicotine EC

\begin{tabular}{|c|c|c|c|c|c|c|}
\hline \multirow[t]{2}{*}{ Outcomes } & \multicolumn{2}{|c|}{ Anticipated absolute effects* $(95 \% \mathrm{Cl})$} & \multirow{2}{*}{$\begin{array}{l}\text { Relative effect } \\
(95 \% \mathrm{Cl})\end{array}$} & \multirow{2}{*}{$\begin{array}{l}\text { № of partici- } \\
\text { pants } \\
\text { (studies) }\end{array}$} & \multirow{2}{*}{$\begin{array}{l}\text { Certainty of } \\
\text { the evidence } \\
\text { (GRADE) }\end{array}$} & \multirow[t]{2}{*}{ Comments } \\
\hline & $\begin{array}{l}\text { Risk with non- } \\
\text { nicotine EC }\end{array}$ & Risk with Nicotine EC & & & & \\
\hline \multirow{2}{*}{$\begin{array}{l}\text { Smoking cessation at 6-12 months } \\
\text { Assessed with biochemical validation }\end{array}$} & \multicolumn{2}{|l|}{ Study population } & \multirow{2}{*}{$\begin{array}{l}\text { RR } 1.70 \\
\text { (1.03 to } 2.81 \text { ) }\end{array}$} & \multirow{2}{*}{$\begin{array}{l}1057 \\
\text { (4 RCTs) }\end{array}$} & \multirow{2}{*}{$\begin{array}{l}\oplus \oplus \oplus \ominus \\
\text { MODERATEa, b }\end{array}$} & \multirow[t]{2}{*}{-} \\
\hline & 6 per 100 & $\begin{array}{l}10 \text { per } 100 \\
(6 \text { to } 17)\end{array}$ & & & & \\
\hline \multirow[t]{2}{*}{ Adverse events at 1 week to 6 months } & \multicolumn{2}{|l|}{ Study population } & \multirow{2}{*}{$\begin{array}{l}\text { RR } 1.01 \\
\text { (0.91 to } 1.11)\end{array}$} & \multirow{2}{*}{$\begin{array}{l}601 \\
\text { (3 RCTs) }\end{array}$} & \multirow{2}{*}{$\begin{array}{l}\oplus \oplus \oplus \ominus \\
\text { MODERATEC }\end{array}$} & \multirow[t]{2}{*}{-} \\
\hline & 35 per 100 & $\begin{array}{l}35 \text { per } 100 \\
\text { (31 to } 38)\end{array}$ & & & & \\
\hline \multirow{2}{*}{$\begin{array}{l}\text { Serious adverse events at } 1 \text { week to } 1 \text { year } \\
\text { Assessed via self-report and medical records }\end{array}$} & \multicolumn{2}{|l|}{ Study population } & \multirow{2}{*}{$\begin{array}{l}\text { RR } 0.60 \\
\text { (0.15 to } 2.44 \text { ) }\end{array}$} & \multirow{2}{*}{$\begin{array}{l}494 \\
\text { (4 RCTs) }\end{array}$} & \multirow{2}{*}{$\begin{array}{l}\oplus \oplus \odot \odot \\
\text { LOWd }\end{array}$} & \multirow{2}{*}{$\begin{array}{l}3 \text { studies reported } \\
\text { no events; effect } \\
\text { estimate based on } \\
\text { the one study in } \\
\text { which events were } \\
\text { reported }\end{array}$} \\
\hline & 2 per 100 & $\begin{array}{l}1 \text { per } 100 \\
(0 \text { to } 4)\end{array}$ & & & & \\
\hline
\end{tabular}

${ }^{\star}$ The risk in the intervention group (and its $95 \%$ confidence interval) is based on the assumed risk in the comparison group and the relative effect of the intervention (and its $95 \% \mathrm{CI}$ ). For cessation, the assumed risk in the control group is based on receipt of moderate-intensity behavioral stop-smoking support. The assumed risk for adverse events and serious adverse events is a weighted mean average of quit rates across control groups in contributing studies.

Cl: Confidence interval; $\mathbf{R C T}$ : randomized controlled trial; RR: Risk ratio 
High certainty: We are very confident that the true effect lies close to that of the estimate of the effect

Moderate certainty: We are moderately confident in the effect estimate: The true effect is likely to be close to the estimate of the effect, but there is a possibility that it is substantially different

Low certainty: Our confidence in the effect estimate is limited: The true effect may be substantially different from the estimate of the effect

Very low certainty: We have very little confidence in the effect estimate: The true effect is likely to be substantially different from the estimate of effect

a Not downgraded for risk of bias. One of three studies considered high risk of bias; removing this study increased the direction of the effect in favor of the intervention.

bDowngraded one level due to imprecision; confidence intervals incorporate no clinically-significant difference as well as clinically-significant benefit.

cDowngraded one level due to imprecision: though confidence intervals are narrow, only 3 studies with 601 participants contribute data.

dDowngraded two levels due to imprecision: confidence intervals encompass clinically-significant harm as well as clinically-significant benefit.

\section{Summary of findings 3. Nicotine EC compared to behavioral support only/no support for smoking cessation}

\section{Nicotine EC compared to behavioral support only/no support for smoking cessation}

Patient or population: People who smoke

Setting: Canada, Italy, UK, USA

Intervention: Nicotine EC

Comparison: Behavioral support only/no support

\begin{tabular}{|c|c|c|c|c|c|c|}
\hline \multirow[t]{2}{*}{ Outcomes } & \multicolumn{2}{|c|}{ Anticipated absolute effects ${ }^{\star}(95 \% \mathrm{Cl})$} & \multirow{2}{*}{$\begin{array}{l}\text { Relative effect } \\
(95 \% \mathrm{CI})\end{array}$} & \multirow{2}{*}{$\begin{array}{l}\text { № of partici- } \\
\text { pants } \\
\text { (studies) }\end{array}$} & \multirow{2}{*}{$\begin{array}{l}\text { Certainty of } \\
\text { the evidence } \\
\text { (GRADE) }\end{array}$} & \multirow[t]{2}{*}{ Comments } \\
\hline & $\begin{array}{l}\text { Risk with behav- } \\
\text { ioral support on- } \\
\text { ly/no support }\end{array}$ & Risk with Nicotine EC & & & & \\
\hline \multirow{2}{*}{$\begin{array}{l}\text { Smoking cessation at } 6 \text { to } 12 \text { months } \\
\text { Assessed using biochemical validation }\end{array}$} & Study population & & \multirow{2}{*}{$\begin{array}{l}\text { RR } 2.70 \\
\text { (1.39 to } 5.26)\end{array}$} & \multirow{2}{*}{$\begin{array}{l}2561 \\
\text { (5 RCTs) }\end{array}$} & \multirow{2}{*}{$\begin{array}{l}\oplus \odot \odot \odot \\
\text { VERY LOWa, b }\end{array}$} & \multirow[t]{2}{*}{-} \\
\hline & 4 per 100 & $\begin{array}{l}11 \text { per } 100 \\
(6 \text { to } 21)\end{array}$ & & & & \\
\hline \multirow{2}{*}{$\begin{array}{l}\text { Adverse events at } 12 \text { weeks to } 6 \text { months } \\
\text { Assessed via self-report }\end{array}$} & \multicolumn{2}{|l|}{ Study population } & \multirow{2}{*}{$\begin{array}{l}\text { RR } 1.22 \\
\text { (1.12 to } 1.32)\end{array}$} & \multirow{2}{*}{$\begin{array}{l}765 \\
\text { (4 RCTs) }\end{array}$} & \multirow{2}{*}{$\begin{array}{l}\oplus \oplus \odot \odot \\
\text { LOWa }\end{array}$} & \multirow[t]{2}{*}{-} \\
\hline & 60 per 100 & $\begin{array}{l}73 \text { per } 100 \\
\text { ( } 67 \text { to } 79)\end{array}$ & & & & \\
\hline \multirow[t]{2}{*}{$\begin{array}{l}\text { Serious adverse events at } 4 \text { weeks to } 6 \\
\text { months }\end{array}$} & Study population & & \multirow[t]{2}{*}{$\begin{array}{l}\text { RR } 1.17 \\
\text { (0.33 to } 4.09 \text { ) }\end{array}$} & \multirow[t]{2}{*}{$\begin{array}{l}1011 \\
\text { (6 RCTs) }\end{array}$} & \multirow{2}{*}{$\begin{array}{l}\oplus \odot \odot \ominus \\
\text { VERY LOWa, c }\end{array}$} & \multirow{2}{*}{$\begin{array}{l}4 \text { of the } 6 \text { stud- } \\
\text { ies reported } \\
\text { no SAEs; MA } \\
\text { is based on } \\
\text { pooled results } \\
\text { from } 2 \text { studies }\end{array}$} \\
\hline & 1 per 100 & $\begin{array}{l}1 \text { per } 100 \\
(0 \text { to } 5)\end{array}$ & & & & \\
\hline
\end{tabular}


*The risk in the intervention group (and its $95 \%$ confidence interval) is based on the assumed risk in the comparison group and the relative effect of the intervention (and its $95 \% \mathrm{Cl}$ ). For cessation, the assumed risk in the control group is based on receipt of limited stop-smoking support. The assumed risk for adverse events and serious adverse events is a weighted mean average of quit rates across control groups in contributing studies.

Cl: Confidence interval; RCT: randomized controlled trial; RR: Risk ratio

\section{GRADE Working Group grades of evidence}

High certainty: We are very confident that the true effect lies close to that of the estimate of the effect

Moderate certainty: We are moderately confident in the effect estimate: The true effect is likely to be close to the estimate of the effect, but there is a possibility that it is substantially different

Low certainty: Our confidence in the effect estimate is limited: The true effect may be substantially different from the estimate of the effect

Very low certainty: We have very little confidence in the effect estimate: The true effect is likely to be substantially different from the estimate of effect

aDowngraded two levels due to risk of bias. Due to lack of blinding and differential support between arms, judged to be at high risk of bias.

bDowngraded one level due to imprecision; although confidence intervals are consistent with clinically-important difference, event count is very low (<100).

cDowngraded two levels due to imprecision; confidence intervals incorporate clinically-significant benefit and clinically-significant harm. 


\section{B A C K G R O U N D}

Throughout this review, we discuss (1) conventional cigarettes and; (2) electronic cigarettes, defined as handheld electronic vaping devices that produce aerosol for inhalation formed by heating an e-liquid. In this review, all mention of smoking, smoking cessation, cigarette use, smoke intake, etc. concern combustible tobacco cigarettes. When the text concerns electronic cigarettes we use the abbreviation 'ECs'. EC users are sometimes described as vapers, and EC use as vaping. We refer to ECs that do not contain nicotine as non-nicotine ECs; these can also be conceptualized as placebo ECs, but we are using the term non-nicotine EC, as they can be conceptualized as an intervention in themselves. This review does not address the use of vaping devices to inhale substances other than nicotine, such as cannabis.

\section{Description of the condition}

Stopping smoking is associated with large health benefits. Despite most people who smoke wanting to quit, many find it difficult to succeed in the long term. Almost half who try to quit without support will not manage to stop for even a week, and fewer than five per cent remain abstinent at one year after quitting (Hughes 2004).

Behavioral support and medications such as nicotine patches or gum increase the chances of quitting through providing nicotine to help alleviate withdrawal symptoms, but even with this additional support long-term quit rates remain low (Cahill 2016; HartmannBoyce 2018b; Hartmann-Boyce 2019). One of the limitations of current treatments is that, despite substituting nicotine delivery, none adequately addresses the sensory, behavioral and social aspects of smoking that ex-smokers miss when they stop smoking (e.g. holding a cigarette in their hands, taking a puff, enjoyment of smoking, feeling part of a group). ECs may offer a way to overcome this limitation (Notley 2018b).

There is no doubt that people become dependent on tobacco, and find it difficult to stop smoking, primarily because of nicotine and its actions on the brain's reward system (Balfour 2004). However, other factors also contribute to tobacco dependence (Benowitz 2010; Rose 2006). Sensory and behavioral cues provide additional reinforcement of smoking behavior (Rose 1993; Rose 2000) and over time become almost as rewarding as nicotine. There are several lines of evidence to support this. Firstly, people who smoke appear to have a preference for cigarette smoke compared to other forms of nicotine delivery. This is partly related to the speed of nicotine delivery through smoke inhalation. However, even when nicotine is administered intravenously it does not provide the same level of satisfaction or reward as smoking (Rose 2000; Westman 1996). Secondly, the local sensory effects of smoking (e.g. the 'scratch' in the back of the throat) may be important for enjoyment and reward. Numbing the sensations of cigarette smoke by anaesthetizing the upper and lower respiratory tract leads to less enjoyment of smoking (Rose 1985). Conversely, products that mimic the sensory effects of smoking on the mouth and throat (such as citric acid, black pepper, and ascorbic acid) reduce craving and some withdrawal symptoms, at least in the short term (Levin 1993; Rose 1994; Westman 1995). Thirdly, very low nicotine content cigarettes (VLNCs) which have a very low content of nicotine (e.g. $0.08 \mathrm{mg}$ instead of the normal $1 \mathrm{mg}$ ) and so have negligible or no central effects, have also been investigated for their role in aiding smoking cessation (Przulj 2013). Despite delivering low levels of nicotine, VLNCs are satisfying over the initial few days of abstinence from nicotine (Donny 2007; Donny 2015; Pickworth 1999; Rose 2000). They also reduce tobacco withdrawal symptoms, including urges to smoke and low mood (Barrett 2010; Donny 2009; McRobbie 2016; Perkins 2010; Rose 2000), and have been shown to improve long-term continuous abstinence rates in one study (Walker 2012). Social aspects of smoking, such as feeling part of a like-minded group, or including smoking behavior as part of one's social identity are also key elements of cigarette smoking that people who smoke report to be key aspects of cigarette dependence (Notley 2018a).

Considering the other factors that contribute to tobacco dependence, there is interest in developing smoking-cessation products that would not only help relieve the unpleasant effects of nicotine withdrawal but would also act as an effective substitute for smoking behavior and the rituals and sensations that accompany smoking, without the health risks associated with the inhalation of tobacco smoke. Until recently the only pharmaceutical treatments available that had some of these characteristics were the nicotine inhalator and nicotine oral spray. However, these do not have greater cessation efficacy than the other nicotine replacement therapy (NRT) products (Hajek 1999; Hartmann-Boyce 2018a). This may in part be due to the considerable effort (e.g. 20 minutes of continuous puffing) needed to provide nicotine blood concentrations consistent with other NRTs (Schneider 2001). Adherence to correct use of the inhalator is low compared to other NRTs (Hajek 1999). It is therefore possible that any advantage of sensorimotor replacement is diminished by low nicotine delivery and limited similarities between inhalator use and sensations of smoking (Bullen 2010). A nicotine inhaler using pressurized air has recently been approved as a smoking cessation aid in the UK. The nicotine delivery is substantially lower than from cigarettes, and also lower than from the nicotine inhalator (Romeu 2020).

\section{Description of the intervention}

ECs are electronic vaping devices that are handheld and produce an aerosol formed by heating an e-liquid, designed for inhalation by the user (E-cigarette ontology 2021). The e-liquid, usually comprising propylene glycol and glycerol, with or without nicotine and flavors, is stored in disposable or refillable cartridges or a reservoir or 'pod'. The commonly-used term for this aerosol is vapor, which we use throughout the review. In many countries, ECs are marketed as consumer products. Although routes are in place for licensing them as a medicine in some areas, no country yet has a licensed, medicinal EC.

ECs provide sensations similar to smoking a cigarette. They provide taste and throat sensations that are closer to smoking than those provided by the nicotine inhalator (Barbeau 2013). The vapor that looks like tobacco smoke is only visible when the user exhales after drawing on the mouthpiece, not when the device is being held. In qualitative studies users report a sense of shared identity with other users, similar to tobacco smoking identity, and also report pleasure and enjoyment of use, suggesting that ECs may be viewed less as a medical cessation aid but rather as an acceptable alternative to tobacco smoking (Cox 2017; Notley 2018a).

There are many different brands and models of EC available. Variation exists both in the device ('product') and consumable (e-liquid used). There is a wide variation in the composition of e-liquids (nicotine content, flavors and other components) (Goniewicz 2012; Goniewicz 2014), with some users choosing to mix their own e-liquids (Cox 2019b). Initial studies showed that early 
models of EC delivered very low amounts of nicotine to naïve users (Bullen 2010; Eissenberg 2010; Vansickel 2010). Later studies that have measured nicotine pharmacokinetics in both experienced and naïve EC users have found that some EC users can achieve blood nicotine levels similar to those achieved with smoking, albeit more slowly, and that their ability to do so often improves over time (Hajek 2015b; Vansickel 2012; Vansickel 2013; Yingst 2019a; Yingst 2019b).

Early on in their development, ECs looked like cigarettes and used disposable cartridges. These models were often called 'cig-alikes'. The nicotine delivery from these products was low, and even the modern versions of EC devices that use pre-filled cartridges, generally produced by the tobacco industry, for the most part have only low nicotine delivery (Hajek 2017). The later refillable, or 'tank', products have a larger battery and a transparent container that users fill with an e-liquid of their choice, and usually provide faster and more efficient nicotine delivery, allow a wider choice of flavors and nicotine concentrations, and are typically used by experienced vapers who manage to switch to vaping completely (ASH 2019; Dawkins 2013b; Farsalinos 2014). Observational evidence suggests people who smoke are more likely to successfully quit using tank models than with cig-a-likes (Chen 2016; Hitchman 2015). EC types are also often grouped by 'generation': first-generation devices are typically cig-a-likes; second-generation devices are usually tank models, sometimes referred to as 'vape pens'; and third-generation devices are tank models which, unlike second-generation devices, allow users to adjust the power (wattage) level of the product (see NCSCT EC briefing for further information and images of different product types). More recently, smaller 'pod' devices, such as Juul, appeared that use nicotine salt. This nicotine formulation reduces irritant effects and allows the delivery of higher nicotine levels that closely mimic the pharmacokinetic profile of nicotine delivery from cigarettes, despite the low battery power of the device (Hajek 2020). Juul has now become the most popular EC in the USA (Huang 2019). The EU Tobacco Products Directive (European Parliament 2014) does not allow sales of e-liquids with nicotine content higher than $20 \mathrm{mg} / \mathrm{ml}$, and so the US version of Juul (59 mg/nl nicotine) is not available within the EU (Huang 2019; Talih 2020).

The different device types (cig-a-like, refillable and pods using high nicotine content salts) may differ significantly in their efficacy in helping people who smoke to quit, as they differ in delivery of nicotine, the active ingredient. Nicotine itself, when delivered through mechanisms and doses similar to that delivered in traditional NRT, is not considered harmful (Hartmann-Boyce 2018a). The safety profile of the different types of EC may be similar as they use the same constituents, although within the generic range of EC types, there is some evidence to suggest EC providing less nicotine may pose higher risks. This is because lownicotine delivery devices need to be puffed with higher intensity to provide users with the nicotine levels that they seek, and more intensive puffing is accompanied by increased inhalation of potential toxicants (Dawkins 2016; Dawkins 2018; Smets 2019). Throughout this review we refer to a nicotine-containing EC as 'nicotine EC' and to nicotine-free EC as 'non-nicotine EC', which can also be considered 'placebo EC'. The 'placebo' comparison is a test just of the nicotine effect and not of the potential sensorimotor or behavioral and social replacement that the EC may provide.

There is no one agreed classification system for EC devices, and product development has moved so quickly that the definitions used within trials of the devices tested may no longer be necessarily fit for purpose. In this review, the definitions used are based on those drawn from the included trials. We currently label three different types of EC as 'cartridges' for devices with disposable cartridges and - typically, but not always - low nicotine delivery (e.g. cig-a-likes); refillable ECs for devices that vapers fill with their own choice of e-liquids; and pods for the small devices that use nicotine salts. We may review this categorization system in future versions of the review as new trials and devices emerge.

\section{Why it is important to do this review}

Since ECs appeared on the market in 2006 there has been a steady increase in their use. In the UK the ASH 2019 survey found 19.4\% of the adult population have ever tried vaping, but only $7.2 \%$ were current vapers. EC use remains slightly more common among men compared with women, although the difference is small. EC use is most prevalent in current (19.9\%) and former (11.6\%) smokers. Less than one per cent of never-smokers report regular EC use. Prevalence data from the USA in 2019 showed that $4.4 \%$ of adults were current EC users (Du 2020). Data from lower-income countries suggest similar levels of EC use and awareness (Besaratinia 2019; Jiang 2016; Palipudi 2016).

Particular concern has been raised about the increased use of EC in young people, especially among never-smokers. Data for 2019 from Canada, England, and the USA show regular use ( $\geq 20$ days in the last 30 days) among 16 - to 19 -year-olds to be $5.7 \%, 2.7 \%$ and $6.7 \%$, respectively. There appear to be some regional differences in the change in the prevalence of EC use. For example, in North America the rates of regular EC use among 16- to 19-year-old never-smokers has significantly increased between 2017 and 2019, compared to England where there has not been any significant change $(0.2 \%$ to $0.3 \%$ ) (Hammond 2020). However, as with adults, regular use is greatest among those who are also smoking and lowest among never-smokers (1.0\%, 0.3\%, and $1.8 \%$ for Canada, England and USA, respectively).

Regulatory approaches being used for ECs currently vary widely, from no regulation to partial and complete bans (McNeill 2021). Within the USA, for example, the Food and Drug Administration (FDA) has classified them as tobacco products and there are a range of laws that include prohibition of EC use indoors, require retailers to have a license to sell, and prohibit sales to minors. Laws prohibiting sales to minors apply nationwide, but other laws vary by state (Du 2020). The European Union includes ECs in their Tobacco Products Directive, except where therapeutic claims are made or in instances where they contain over $20 \mathrm{mg} / \mathrm{nl}$ of nicotine, when they will require medicines authorization (European Parliament 2014).

Categorical statements about the toxicity of ECs are not possible because of the large number of devices and liquids available and the frequent addition of new products to the market. In 2019, cases of severe lung injury associated with EC use were reported in the USA, and by February 2020 there were around 2800 hospitalized cases or deaths (CDC 2020). This illness was termed E-cigarette or Vaping-Associated Lung Injury (EVALI) and caused concern throughout the world (Hall 2020), and a negative change in people's perception of the risks of EC use compared to smoking (Tattan-Birch 2020). These cases were somewhat at odds with data from trials and cohort studies, and it was later found that these injuries were related to use of tetrahydrocannabinol (THC)-containing EC, and in 
particular THC products adulterated with vitamin E acetate (Blount 2020; Hartnett 2020). Among those brands of nicotine EC that have been tested, levels of toxins have been found to be substantially lower than in cigarettes (Hajek 2014; McNeill 2021). Long-term effects beyond 12 months are unknown, although based on what is known about liquid and vapor constituents and patterns of use, a report from the UK's Royal College of Physicians has concluded that using an EC is likely to be considerably safer than smoking (RCP 2016). The US National Academies of Sciences, Engineering, and Medicine (NASEM) concluded that ECs are likely to be far less harmful than continuing to smoke cigarettes, with the caveat that the long-term health effects of e-cigarette use are not yet known (NASEM 2018).

Despite general acknowledgement that EC use exposes the user to fewer toxicants and at lower levels than smoking cigarettes (McNeill 2021; NASEM 2018; RCP 2016), there remains some hesitancy in making these products available to people who smoke as a harm reduction tool or smoking cessation aid (e.g. McDonald 2020). Lack of quality control measures, possible harms of second-hand EC vapor inhalation, concerns that the products may be a gateway to smoking initiation or may prolong continued dual-use of tobacco, concerns that ECs may undermine smoke-free legislation if used in smoke-free spaces, concerns about the involvement of the tobacco industry, and concerns that the long-term effects of EC use on health are not yet known are often cited. Recently, a report from the US Preventive Services Taskforce concluded "that the current evidence is insufficient to assess the balance of benefits and harms of electronic cigarettes (e-cigarettes) for tobacco cessation in adults" (USPFTS 2021). However, others suggest that potential benefits outweigh potential disadvantages (Farsalinos 2014; Hajek 2014; McNeill 2021; NASEM 2018; RCP 2016).

People who smoke, healthcare providers and regulators are interested to know if ECs can help smokers quit and if it is safe to use them to do so. In particular, healthcare providers have an urgent need to know what they should recommend for people who want to stop smoking. The largest health gains are achieved from stopping smoking completely, as opposed to reducing cigarette consumption, and as such this review focuses on the effectiveness of ECs in aiding smoking cessation.

This review was first published in 2014, and updated in 2016 and 2020.

Following the publication of the 2020 update of this review, we are maintaining it as a living systematic review (Brooker 2019). This means we are continually running searches and incorporating new evidence into the review. For more information about the living systematic review methods being used, see Appendix 1. A living systematic review approach is appropriate for this review, for three reasons. First, the review addresses an important public health issue; the role of ECs in enabling people who smoke to stop smoking, with potential for substantial ongoing individual and societal benefits if effective. Secondly, there remains uncertainty in the existing evidence; more studies are needed to confirm the degree of benefit for different comparisons and product types, and there is considerable uncertainty about adverse events and other markers of safety. Thirdly, we are aware of multiple ongoing trials on this topic that are likely to have an important impact on the conclusions of the review.

\section{O B JECTIVES}

To examine the safety, tolerability and effectiveness of using electronic cigarettes (ECs) to help people who smoke achieve longterm smoking abstinence.

\section{METHODS}

\section{Criteria for considering studies for this review}

\section{Types of studies}

We include randomized controlled trials (RCTs) and randomized cross-over trials in which people who smoke are randomized to ECs or to a control condition. RCTs are the best available primary evidence, but the continued paucity of RCTs in this area requires that we also include uncontrolled intervention studies in which all participants are given an EC intervention.

We include studies regardless of their publication status or language of publication.

\section{Types of participants}

People defined as currently smoking cigarettes at enrolment into the studies. Participants could be motivated or unmotivated to quit.

\section{Types of interventions}

Any type of EC or intervention intended to promote EC use for smoking cessation, including studies which did not measure smoking cessation but provided ECs with the instruction they be used as a complete substitute for cigarette use. ECs may or may not contain nicotine.

\section{Types of comparators}

We compare nicotine ECs with non-nicotine ECs, ECs versus alternative smoking cessation aids, including NRT or no intervention, and ECs added to standard smoking cessation treatment (behavioral or pharmacological or both) with standard treatment alone.

\section{Types of outcome measures}

\section{Primary outcomes}

- Cessation at the longest follow-up point, at least six months from the start of the intervention, measured on an intention-totreat basis using the strictest definition of abstinence, preferring biochemically-validated results where reported

- Number of participants reporting adverse events or serious adverse events at one week or longer (as defined by study authors)

\section{Secondary outcomes}

Changes in the following measures at one week or longer:

- Carbon monoxide (CO), as measured through breath or blood

- Blood pressure

- Heart rate

- Blood oxygen saturation

- Lung function measures

- Known toxins/carcinogens, as measured through blood or urine (toxicant names and abbreviations are listed in Appendix 2) 
Studies had to report one of the primary or secondary outcomes above to be eligible for inclusion.

\section{Search methods for identification of studies}

\section{Electronic searches}

For this update we searched the following databases on 1st February 2021:

\section{- Cochrane Tobacco Addiction Group Specialized Register}

- Cochrane Central Register of Controlled Trials (CENTRAL)

- MEDLINE (OVID SP)

- Embase (OVID SP)

- PsycINFO (OVID SP)

- ClinicalTrials.gov

- WHO International Clinical Trials Registry Platform (ICTRP: www.who.int/ictrp/en/)

At the time of the search, the Register included the results of searches of the Cochrane Central Register of Controlled trials (CENTRAL), issue 1, 2021; MEDLINE (via OVID) to update 20210104; Embase (via OVID) to week 202101; PsycINFO (via OVID) to update 20201228. See the Tobacco Addiction Group website for full search strategies and a list of other resources searched.

For the first version of the review we also searched CINAHL (EBSCO Host) (2004 to July 2014). We did not search this database from 2016 onwards as it did not contribute additional search results to the first version of the review. The search terms were broad and included e-cig\$ OR elect\$ cigar\$ OR electronic nicotine. The search for the 2016 update added the terms vape or vaper or vapers or vaping. The 2020 searches added further terms, including the MESH heading 'Electronic Nicotine Delivery Systems' and terms to limit by study design. Our search strategy for MEDLINE (Ovid SP) is listed in Appendix 3. The previously-used search strategy is shown in Appendix 4. The search date parameters of the original searches were limited to 2004 to the present, due to the fact that ECs were not available before 2004 .

\section{Searching other resources}

We searched the reference lists of eligible studies found in the literature search and contacted authors of known trials and other published EC studies.

\section{Data collection and analysis}

\section{Selection of studies}

Two review authors (for this update from: JHB, NL, CN, RB, $\mathrm{PH}, \mathrm{NR}, \mathrm{ARB}, \mathrm{HMR}$ ) independently prescreened all titles and abstracts obtained from the search, using a screening checklist, and then independently screened full-text versions of the potentially relevant papers for inclusion. We resolved any disagreements by discussion or with a third review author.

\section{Data extraction and management}

Two review authors (for this update from: CN, ARB, AT, HMR) extracted data from the included studies using a pre-piloted data extraction form, and checked them against each other. We resolved any disagreements by discussion or with a third review author. We extracted data on:
- Author

- Date and place of publication

- Study dates

- Study design

- Inclusion and exclusion criteria

- Setting

- Summary of study participant characteristics

- Summary of intervention and control conditions

- Number of participants in each arm

- Smoking cessation outcomes

- Type of biochemical validation (if any)

- Adverse events (AEs), serious adverse events (SAEs), and relevant biomarkers

- Assessment time points

- Study funding source

- Author declarations of interest

- Risk of bias in the domains specified below

- Additional comments

We adopted a broad focus to detect a variety of adverse events.

One review author (JHB) then entered the data into Review Manager 2020 software for analyses, and another checked them (AB for this update).

\section{Assessment of risk of bias in included studies}

Two review authors (for this update from: CN, ARB, AT, HMR) independently assessed the risks of bias for each included study, using the Cochrane 'Risk of bias' Tool v1 (Higgins 2011). This approach uses a domain-based evaluation that addresses seven different areas: random sequence generation; allocation concealment; blinding of participants and providers; blinding of outcome assessment; incomplete outcome data; selective outcome reporting; and other potential sources of bias. We assigned a grade (low, high, or unclear) for risk of bias for each domain. We resolved disagreements by discussion or by consulting a third review author.

Specific considerations about judgments for individual domains in this review are outlined below:

- Random sequence generation/allocation concealment: We rated all non-randomized studies at high risk in these domains;

- Blinding of participants and personnel: We did not evaluate this domain for non-randomized studies, as we considered it not to be applicable. For randomized studies which did not use blinding, we considered studies to be at low risk in this domain if the intervention was compared to an active control of similar intensity, as we judged performance bias to be unlikely in this circumstance. If studies were unblinded and the comparator group was a minimal-intervention control or of lower intensity than the intervention group, we considered the study to be at high risk of bias in this domain;

- Following standard methods of the Cochrane Tobacco Addiction Review Group, we considered studies to be at low risk of detection bias (blinding of outcome assessment) if our primary outcome was objectively measured or if the intensity of intervention was similar between groups, or both. For studies where cessation was measured, our judgment was based on 
whether cessation was biochemically verified. For other studies, we judged this domain based on adverse or serious adverse events;

- Again following standard methods of the Cochrane Tobacco Addiction Group, we rated studies at high risk of attrition bias if loss to follow-up was greater than $50 \%$ overall or if there was a difference in follow-up rates of more than $20 \%$ between study arms.

We judged studies to be at high risk of bias overall if they were rated at high risk in at least one domain, and at low risk of bias overall if they were judged to be at low risk across all domains evaluated. We judged the remaining studies to be at unclear risk of bias overall.

\section{Measures of treatment effect}

We analyzed dichotomous data by calculating the risk ratio (RR). For cessation, we calculated the RR as (number of events in intervention condition/intervention denominator) / (number of events in control condition/control denominator)) with a $95 \%$ confidence interval $(\mathrm{Cl})$, using data at the longest follow-up period reported.

We analyzed continuous data (other measures of tobacco exposure) by comparing the difference between the mean change from baseline to follow-up in the intervention and comparator groups. For outcomes other than cessation where data were reported at multiple time points, we used data at the longest follow-up point at which ECs were still being provided.

\section{Unit of analysis issues}

In the case of trials with multiple arms, we do not combine data between arms unless this is the way it has been presented by study authors. We note in our analyses where this is the case.

For all but one study, the unit of assignment was the individual. Dawkins 2020 assigned condition based on homeless support service; this was a small pilot study with very few events and hence we judged clustering to have very little impact on our overall result. If larger cluster-randomized trials are eligible in the future, we will assess whether study authors have adjusted for this clustering, and whether this had an impact on the overall result. When clustering appears to have had little impact on the results, we will use unadjusted quit-rate data; however when clustering does appear to have an impact on results, we will adjust for this using the intraclass correlation (ICC).

For randomized cross-over trials, we report results at the end of the first assignment period where available and where sufficiently long to meet our inclusion criteria for outcomes. All other outcomes from randomized cross-over trials are reported narratively. We offer a narrative synthesis of data from non-randomized studies, and where possible use effect direction plots as described in the Cochrane Handbook (Higgins 2021).

\section{Dealing with missing data}

For smoking cessation, we used a conservative approach, as is standard for the Cochrane Tobacco Addiction Group, treating participants with missing data as still smoking. We based the proportion of people affected by adverse events on the number of people available for follow-up, and not the number randomized.

Electronic cigarettes for smoking cessation (Review)

Copyright (c) 2021 The Cochrane Collaboration. Published by John Wiley \& Sons, Ltd.
For other outcomes, we use complete-case data and do not attempt to impute missing values.

\section{Assessment of heterogeneity}

We assessed the clinical and methodological diversity between studies to guide our decision as to whether data should be pooled. We were also guided by the degree of statistical heterogeneity, assessed by calculating the 12 statistic (Higgins 2003), and considering a value greater than $50 \%$ as evidence of substantial heterogeneity. We did not present pooled results where $\mathrm{I}^{2}$ values exceeded $75 \%$.

\section{Assessment of reporting biases}

Reporting bias can be assessed using funnel plots, where 10 or more RCTs contribute to an outcome. However, there are currently insufficient studies to support this approach.

\section{Data synthesis}

We provide a narrative summary of the included studies. Where appropriate, we have pooled data from these studies in metaanalyses. For dichotomous data, we used a fixed-effect MantelHaenszel model to calculate the RR with a 95\% confidence interval, in accord with the standard methods of the Cochrane Tobacco Addiction Group for cessation studies.

For continuous outcomes, we pooled mean differences (or standardized mean differences for studies using different measures for the same construct), using the inverse variance approach (also with a $95 \% \mathrm{Cl}$ ).

\section{Subgroup analysis and investigation of heterogeneity}

We had planned to undertake subgroup analyses to investigate differences between studies, such as:

- Intensity of behavioral support used;

- Type of EC (cartridge; refillable; pod);

- Instructions for EC use (e.g. study provision, length of provision, whether participants had a role in product choice);

- Type of participants (e.g. experience of EC use).

However, there were too few studies to conduct such analyses. Should further studies become available in future, we will follow this approach. For safety outcomes, we present subgroups by length of follow-up for descriptive purposes.

In the absence of sufficient data for subgroup analyses on EC type, in the text we specify the type of nicotine $\mathrm{EC}$ when reporting pooled results for cessation.

\section{Sensitivity analysis}

We conducted sensitivity analyses to detect whether pooled results were sensitive to the removal of studies judged to be at high risk of bias.

\section{Summary of findings and assessment of the certainty of the evidence}

Following standard Cochrane methodology, we created 'Summary of findings' tables for our three main comparisons using GRADEpro GDT: nicotine EC versus non-nicotine EC; nicotine EC versus NRT; and nicotine EC versus behavioral support only/no support. We 
selected these comparisons a priori as being the most clinically relevant. In the 'Summary of findings' tables, we present data on our primary outcomes (cessation, adverse events, serious adverse events) for these main comparisons. Also following standard Cochrane methodology, we used the five GRADE considerations (study limitations, consistency of effect, imprecision, indirectness and publication bias) to assess the certainty of the body of evidence for each outcome, and to draw conclusions about the certainty of evidence within the text of the review.

\section{RES U L T S}

\section{Description of studies}

\section{Results of the search}

For this update, our bibliographic database searches identified 825 non-duplicate records (Figure 1). We screened all records and retrieved the full-text papers of 100 potentially relevant articles. After screening and checking the full-text of 100 papers, we included 26 records, representing six studies new for this update (Czoli 2019; Ikonomidis 2020; Ozga-Hess 2019; Pulvers 2020; Scheibein 2020; Yingst 2020), nine new articles linked to studies already identified, and 11 new references to ongoing studies (see Characteristics of ongoing studies). Secondary study reports, commentaries, and correspondence relating to included studies are linked to studies in the reference section. Figure 2, Figure 3 and Figure 4 present PRISMA flow charts for previous versions of this review. 
Figure 1. 2021 update flow diagram

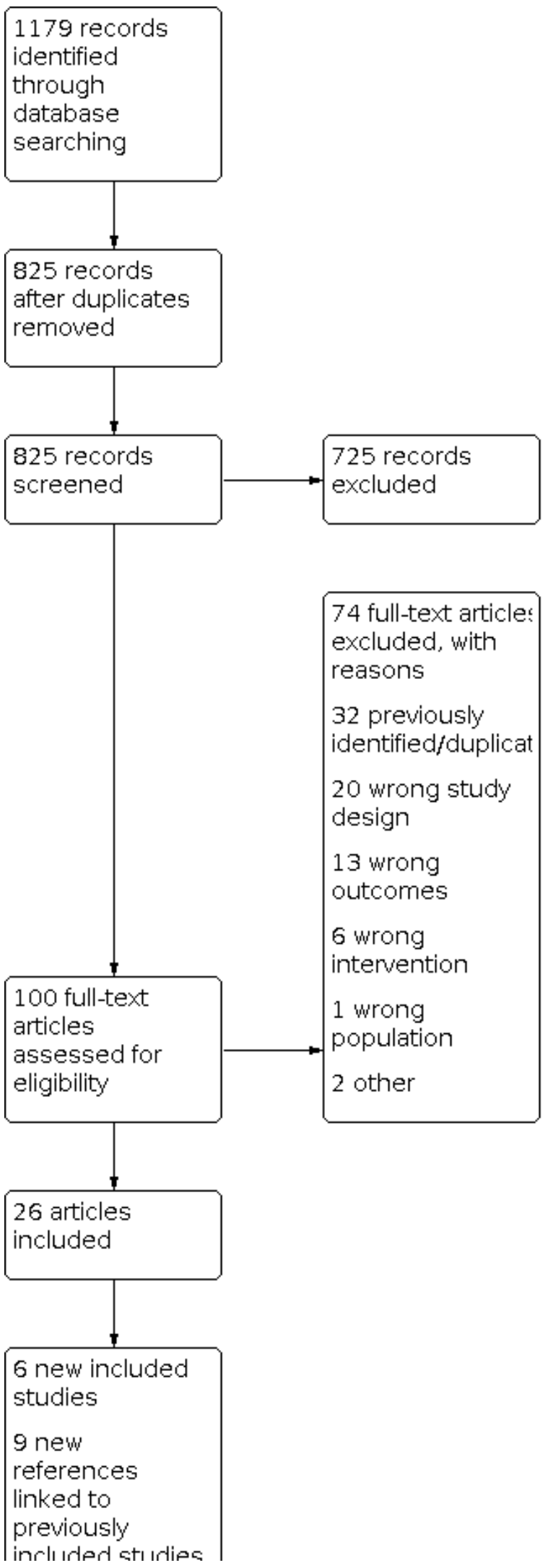


Figure 1. (Continued)

previously
included studies
11 new ongoing
studies

Figure 2. 2020 update flow diagram

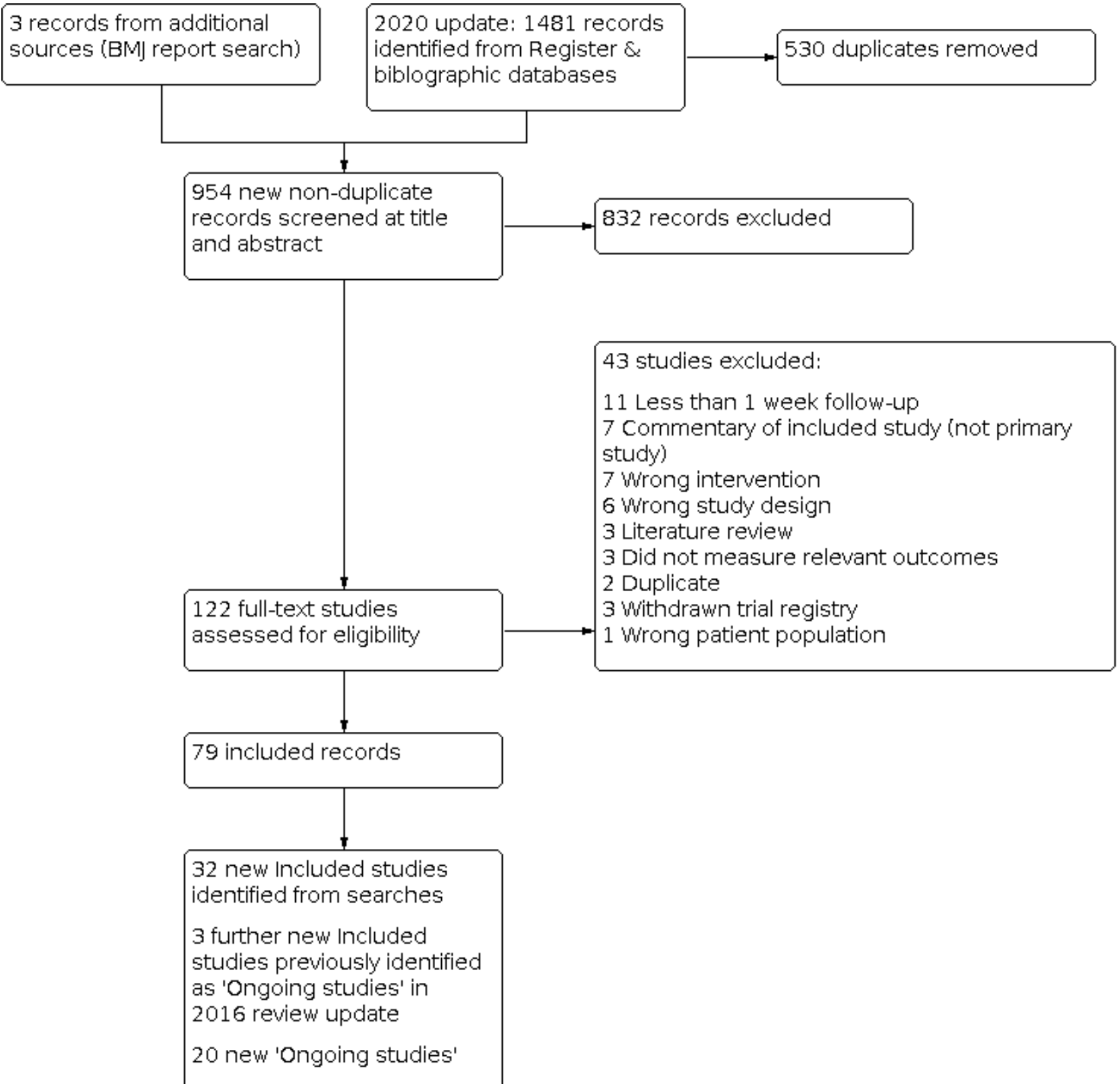




\section{Figure 3. 2016 update flow diagram}

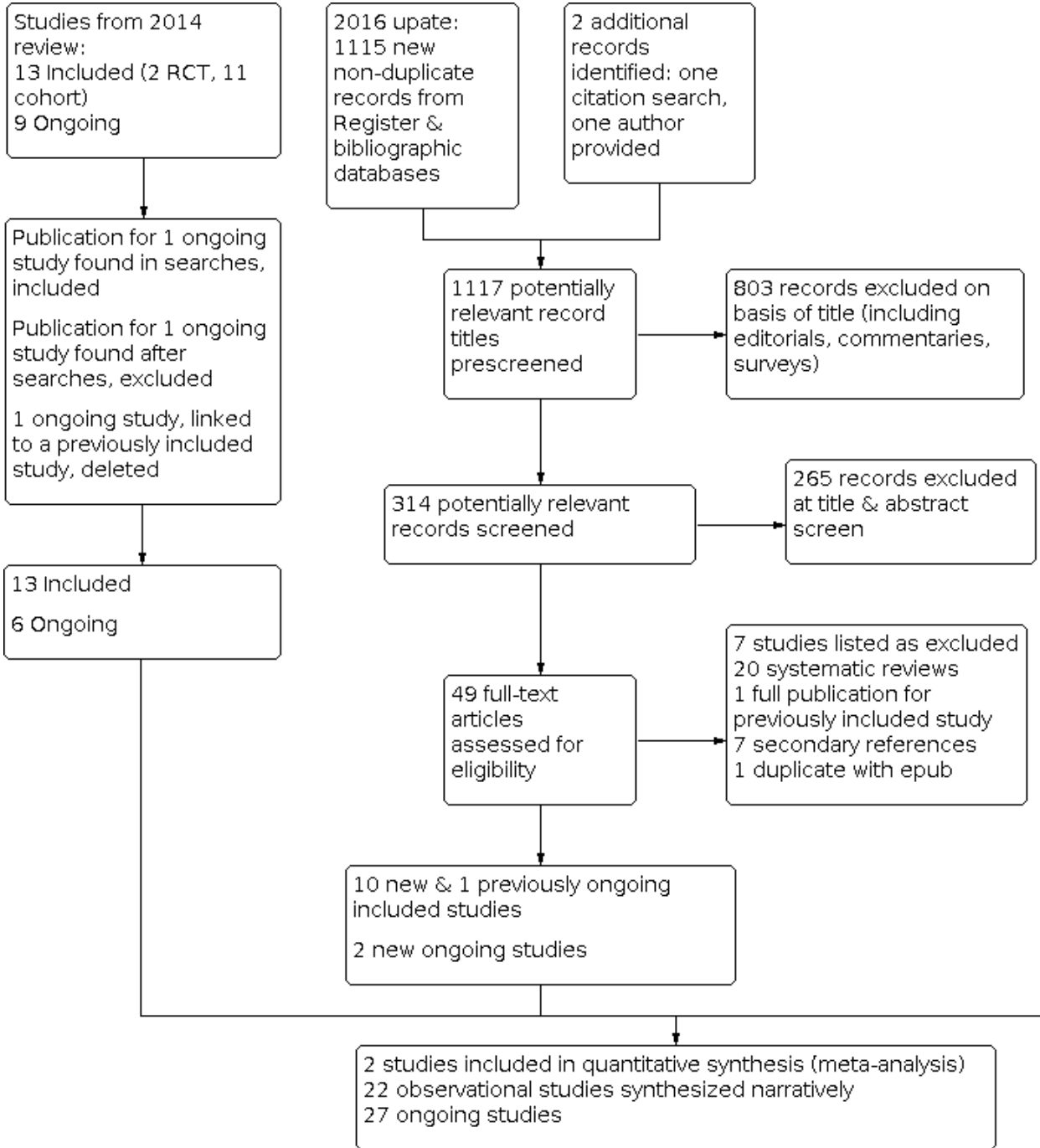

76 new ongoing study records from ct.gov

3 additional records from other registers via WHO portal

16 potentially relevant studies from ct.gov, 60 not relevant

(2 replaced by published protocols found by searching on NCT

number subsequent to database searches)

3 potentially relevant studies from other registers

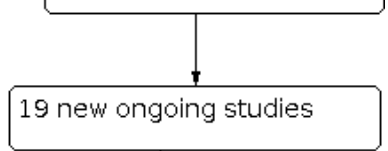


Figure 4. 2014 flow diagram

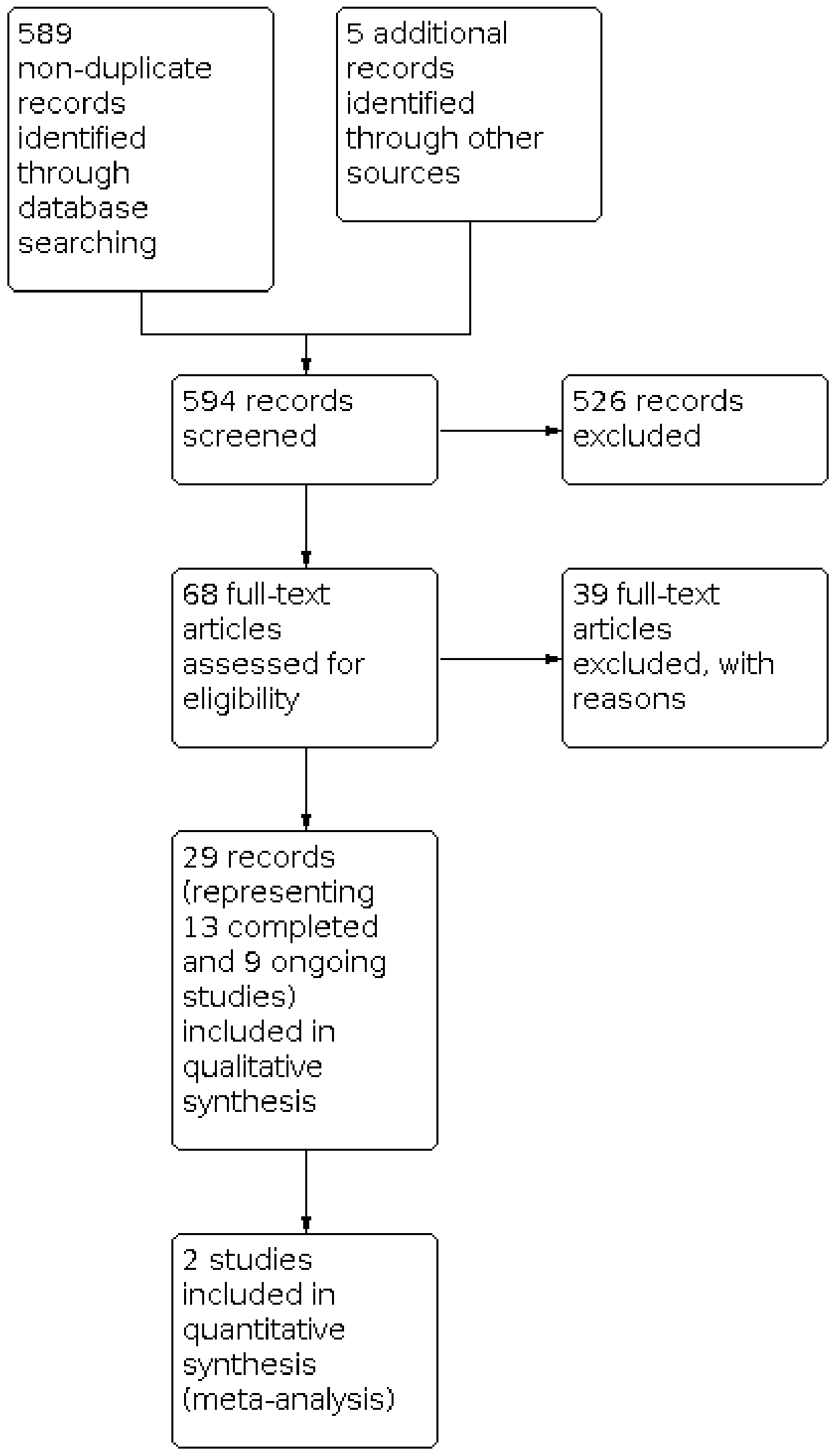




\section{Included studies}

In total, we include 56 studies, six new included studies and 50 eligible included studies identified in previous versions of the review. Key features of the included studies are summarized below. Further details on each included study can be found in the Characteristics of included studies tables.

\section{Participants}

The 56 included studies represented 12,804 participants. Twentyfour studies were conducted in the USA, nine were conducted in the UK, seven in Italy, three in Greece, two each in Australia, New Zealand, and Canada, and one each in Belgium, Ireland, Poland, the Republic of Korea, South Africa, Switzerland, and Turkey. All studies were conducted in adults who smoke. Seventeen studies exclusively recruited participants who were not motivated to quit smoking, and 29 studies exclusively recruited participants motivated to quit; motivation was not specified for the other studies. Eighteen studies recruited from specific population groups; these included six studies which recruited participants based on physical health condition (heart attack, cancer, HIV, periodontitis, awaiting surgery), three studies which recruited participants with serious mental illness, and three studies which recruited participants in treatment or having recently completed treatment for alcohol or other drug use. Two studies recruited people accessing homeless centres or using supported temporary accomodation. One study each recruited: people aged 55 or older, young adults, and black and latinx participants.

\section{Interventions and comparators}

All but one study provided nicotine EC, either alone (50 studies) or in conjunction with NRT or varenicline (five studies). One study recruited dual users at baseline, and instructed them to continue using their own EC devices (Czoli 2019). In two studies where nicotine EC was provided on its own, nicotine levels were judged to be so low as to be clinically comparable to non-nicotine EC (Lee 2019; Van Staden 2013); we include these studies in non-nicotine EC comparisons. Eight studies compared nicotine EC with nonnicotine EC, 16 studies compared nicotine EC to behavioral support only or to no support, and eight studies compared nicotine EC to NRT. One study directly compared a cig-a-like with a refillable (tank) device (Yingst 2020). Results from these studies are reported by comparison in Effects of interventions. Further details on the intervention and comparator groups (where applicable) for each study can be found in the Characteristics of included studies tables.
Where reported in the primary research publications, details on the devices tested can also be found in the Characteristics of included studies tables. Of the studies with sufficient data with which to judge, 26 used cartridge devices (only one of which had high nicotine delivery), 21 used refillable devices, three used both types, one used a pod device, and the remainder did not report device type.

\section{Outcomes}

Of the 56 included studies:

- 22 reported data on abstinence

- 39 reported data on adverse events

- 24 reported data on serious adverse events

- 36 reported data on carbon monoxide

- 9 reported data on heart rate

- 12 reported data on blood pressure

- 2 reported data on blood oxygen saturation

- 9 reported data on at least one known toxin/carcinogen

- 5 reported data on at least one measure of lung function

\section{Study types and funding}

Twenty-nine studies were RCTs, 13 of which contributed to cessation analyses. Five studies used randomized cross-over designs, and the remainder were uncontrolled cohort studies. Of the 46 studies which reported funding information, 32 had no EC industry funding or support.

\section{Excluded studies}

We list 92 studies excluded at full-text stage, along with reasons for exclusion, in the Characteristics of excluded studies table. The most common reason for exclusion was that studies were shortterm, following up participants for periods of less than one week.

\section{Risk of bias in included studies}

Overall, we judged five studies (Bullen 2013; Eisenberg 2020; Hajek 2019; Lee 2018; Lee 2019) to be at low risk of bias, ten to be at unclear risk, and the remaining 41 at high risk of bias (note, this includes the non-randomized studies, which we deemed to be at high risk due to this lack of randomization).

Details of 'Risk of bias' judgments for each domain of each included study can be found in the Characteristics of included studies table. Figure 5 illustrates judgments for each included study. 
Figure 5. Risk of bias summary: review authors' judgements about each risk of bias item for each included study.

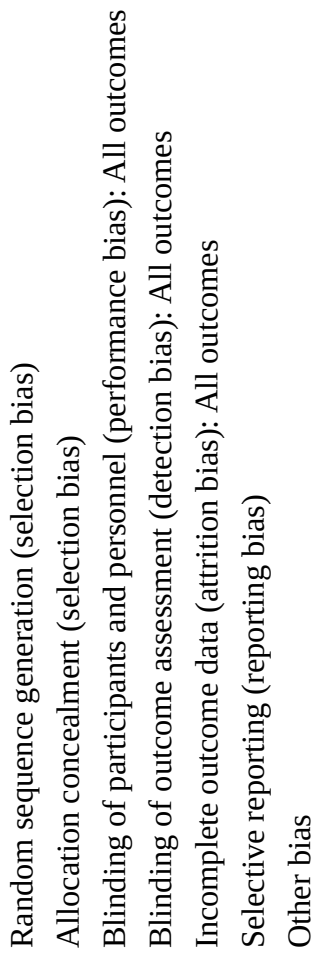

Adriaens 2014

Baldassarri 2018

Bell 2017

Bullen 2013

Caponnetto 2013a

Caponnetto 2013b

Carpenter 2017

Czoli 2019

Dawkins 2020

Eisenberg 2020

Ely 2013

Felicione 2019

George 2019

Goniewicz 2017

Guillaumier 2018

Hajek 2015a

Hajek 2019

Halpern 2018

Hatsukami 2020

Hickling 2019

Holliday 2019

Humair 2014

Ikonomidis 2018

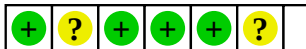

$+?+$

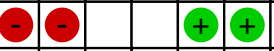


Figure 5. (Continued)

\begin{tabular}{|c|c|c|c|c|c|c|c|}
\hline Humair 2014 & & & & & |? & ? & \\
\hline Ikonomidis 2018 & $?$ & $?$ & - & $?$ & + & $?$ & $?$ \\
\hline Ikonomidis 2020 & + & $?$ & $?$ & + & + & $?$ & \\
\hline Ioakeimidis 2018 & $?$ & $?$ & + & + & $?$ & $?$ & \\
\hline Kumral 2016 & $?$ & $?$ & - & - & $?$ & + & \\
\hline Lee 2018 & + & + & + & + & + & + & \\
\hline Lee 2019 & + & + & + & + & + & + & \\
\hline Lucchiari 2020 & + & + & + & + & + & - & \\
\hline Martner 2019 & & - & & & $?$ & $?$ & \\
\hline McRobbie 2015 & & E & & & + & + & \\
\hline Meier 2017 & $?$ & $?$ & + & + & $?$ & + & \\
\hline NCT02648178 & & - & & & + & $?$ & \\
\hline NCT02918630 & $?$ & $?$ & $?$ & $?$ & $?$ & E & $?$ \\
\hline Nides 2014 & & - & & & + & + & \\
\hline Oncken 2015 & $?$ & $?$ & + & + & + & $?$ & \\
\hline Ozga-Hess 2019 & $?$ & $?$ & & + & C & + & \\
\hline Pacifici 2015 & & & & & + & - & \\
\hline Polosa 2011 & & - & & & + & $?$ & \\
\hline Polosa 2014b & & $(-$ & & & + & $?$ & \\
\hline Polosa 2015 & & - & & & + & $?$ & \\
\hline Pratt 2016 & 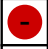 & E & & & + & + & \\
\hline Pulvers 2018 & - & - & & & + & + & \\
\hline Pulvers 2020 & + & + & & + & + & + & \\
\hline Scheibein 2020 & & & & $(-$ & E & $?$ & \\
\hline Smith 2020 & $?$ & $?$ & + & + & + & $?$ & \\
\hline Stein 2016 & 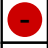 & $=$ & & & + & + & \\
\hline Strasser 2016 & $?$ & $?$ & $?$ & + & - & + & \\
\hline Tseng 2016 & + & $?$ & + & + & + & + & \\
\hline Valentine 2018 & - & - & & & $?$ & $?$ & \\
\hline Van Staden 2013 & 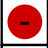 & 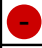 & & & + & $?$ & \\
\hline Veldheer 2019 & + & + & + & + & $?$ & - & \\
\hline Wadia 2016 & 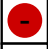 & 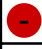 & & & + & + & \\
\hline Walele 2018 & + & + & & & + & + & \\
\hline Walker 2020 & + & + & & + & + & $?$ & \\
\hline Yingst 2020 & $?$ & $?$ & + & + & + & $?$ & \\
\hline
\end{tabular}

\section{Allocation}

We judged 23 studies to be at high risk of selection bias; for 22, this is because the studies were not randomized. We also rated a pilot cluster-randomized trial at high risk as randomization was not carried out as intended for pragmatic reasons (Dawkins 2020). We judged 14 studies to be at low risk of selection bias, and the remainder to be at unclear risk as there was insufficient information with which to judge.

\section{Blinding}

Of the 35 studies assessed for these domains, we judged 16 to be at low risk for both performance and detection bias. We rated 14 at high risk for performance or detection bias, or both. In these studies, blinding was not used and different levels of support were

provided; this alone or in conjunction with the outcome measures being used (subjective rather than objective measures) meant we thought there was a high risk of bias being introduced. We judged the rest to be at unclear risk.

\section{Incomplete outcome data}

We judged most studies (40 out of 56) to be at low risk of attrition bias. We rated six studies with substantial loss to follow-up at high risk of attrition bias. The remainder did not provide sufficient data on which to judge, and hence we judged them to be at unclear risk.

\section{Selective reporting}

Of the 56 studies, we considered that 29 were at low risk of reporting bias, as all prespecified/expected outcomes were reported. We 
rated four at high risk, as data were not presented as specified in the original protocols. We judged the rest to be at unclear risk, due to insufficient information with which to make a judgment.

\section{Other potential sources of bias}

We considered loakeimidis 2018 to be at high risk of other bias; data were from a conference poster and the associated abstract, and quit rates in the intervention arm differed between the two sources.

\section{Effects of interventions}

See: Summary of findings 1 Nicotine EC compared to NRT for smoking cessation; Summary of findings 2 Nicotine EC compared to non-nicotine EC for smoking cessation; Summary of findings 3 Nicotine EC compared to behavioral support only/no support for smoking cessation

Data on our outcomes of interest are summarized below. Due to the volume of data available, some relevant information is hosted on a companion repository; these data are open-access and can be found at https://doi.org/10.5287/bodleian:dX4Dgp7dJ. They are referred to below as supplemental tables. Forest plots are available through 'analysis' links; for some outcomes, benefit is plotted on the right, for others on the left. This is due to direction of effect, e.g. an increase in cessation is a benefit, whereas an increase in a carcinogen is not.

\section{Direct comparisons between nicotine EC and other pharmacotherapies}

Comparisons reported here include cartridge and refillable nicotine ECs versus NRT, and cartridge nicotine ECs versus varenicline. Only randomized controlled trials contribute data.

\section{Cessation}

Pooled data from three studies ( 2 cartridge, 1 refillable), all of which we rated at low risk of bias, showed higher quit rates in people randomized to nicotine EC than to NRT (RR $1.69,95 \% \mathrm{Cl} 1.25$ to 2.27 $\mathrm{I}^{2}=0 \%$; 1498 participants; Analysis 1.1). One study (loakeimidis 2018), available as a conference presentation only and considered at high risk of bias due to inconsistencies in the data reported and an unclear definition of abstinence, found lower quit rates in people allocated to nicotine EC (cartridge) compared to those allocated to varenicline (RR $0.31,95 \% \mathrm{Cl} 0.11$ to $0.82 ; 54$ participants; Analysis 2.1).

\section{Adverse events}

Pooled data from two studies (both considered at low risk of bias) showed no evidence of a difference in the number of participants reporting adverse events (AEs) between nicotine EC and NRT arms (RR $0.98,95 \% \mathrm{Cl} 0.80$ to $1.19 ; \mathrm{I}^{2}=0 \%$; 485 participants; Analysis 1.2). Hajek 2019 did not contribute data to this analysis due to the way in which events were recorded; of their prespecified adverse reactions of interest, nausea was more frequent in the NRT group, throat/mouth irritation was more frequent in the nicotine EC group, and there was little difference in other reactions (see Supplemental Table 1 for more detail).

In loakeimidis 2018, reports of sleep disorders were evenly distributed between groups, and nausea was more common in the varenicline arm than in the nicotine EC arm (see Supplemental Table 1 for more detail).

\section{Serious adverse events}

Two studies comparing nicotine ECs with NRT provided data on SAEs; in one (Lee 2018) none occurred in either arm. In Hajek 2019 ( $n=698$ ), more events occurred in the nicotine EC arm than in the NRT arm, but the confidence interval was wide and included no difference as well as the possibility of more events in the NRT arm (RR 1.37, 95\% Cl 0.77 to 2.41; Analysis 1.3). As noted above, Bullen 2013, which compared nicotine EC, non-nicotine EC, and NRT, only reported that no serious adverse events (SAEs) occurred that were considered related to study treatment. No events occurred in loakeimidis 2018 (Analysis 2.2).

\section{Carbon monoxide (CO)}

Pooled data from two studies (Hatsukami 2020; Lee 2018; neither considered at high risk of bias) comparing nicotine EC with NRT found that $\mathrm{CO}$ levels decreased more in those randomized to nicotine $\mathrm{EC}$, but the point estimate was small, confidence intervals were wide, and statistical heterogeneity was substantial (MD -0.66 ppm, $95 \% \mathrm{Cl}-1.94$ to $0.62 ; \mathrm{I}=69 \%$; 136 participants; Analysis 1.4 ).

\section{Heart rate, blood pressure, and oxygen saturation}

Only Hatsukami 2020 contributed data for these outcomes. A small benefit in favor of EC was found for change in heart rate (Analysis 1.5). No difference was found for blood pressure or blood oxygen saturation, although confidence intervals were wide (Analysis 1.6; Analysis 1.7).

\section{Toxicants}

Again, only Hatsukami 2020 contributed data for these outcomes. For 3-HPMA, 2-HPMA, HMPMA, PheT, and CEMA, point estimates favored EC but confidence intervals included no difference (Analysis 1.8; Analysis 1.10; Analysis 1.11; Analysis 1.12; Analysis 1.13). Both AAMA and NNAL decreased more in NRT than in EC groups, with confidence intervals excluding no difference (Analysis 1.9; Analysis 1.14).

\section{Lung function}

Lee 2018 measured change in FEV1 and FEV1/FVC; for both outcomes, point estimates favored EC over NRT; confidence intervals excluded no difference for FEV1 (Analysis 1.15; Analysis 1.16).

\section{Nicotine EC alone or versus control}

Comparisons reported here include nicotine EC versus non-nicotine EC, and nicotine EC compared to behavioral support only or to no support. In this section, we also report results from studies in which all participants received nicotine EC (cohort studies and randomized studies which did not differ across arms in EC provision, device generation, or nicotine content).

\section{Cessation}

\section{Randomized controlled trials}

At six months or longer, quit rates were higher in nicotine EC groups than in comparator groups. Compared to EC without nicotine (placebo EC), pooled results showed nicotine EC produced higher quit rates (RR $1.70,95 \% \mathrm{Cl} 1.03$ to $2.81 ; 12=0 \% ; 3$ studies of cartridge devices, 1 refillable, 1057 participants; Analysis 3.1). The effect size increased when we removed the one study at high risk of bias (Lucchiari 2020). The effect was more pronounced when comparing 
nicotine EC to behavioral support only or to no support (RR 2.70, $95 \% \mathrm{Cl} 1.39$ to $5.26 ; \mathrm{I}^{2}=0 \%$; 5 studies ( 3 refillable, 2 cartridge), 2561 participants; Analysis 4.1). As this involved unblinded comparisons with unequal levels of support, we judged all data contributing to this outcome to be at high risk of bias.

Pulvers 2020 (pod device) measured cessation at six months in the intervention group only, using self-report. As they did not measure cessation at six months in the comparator group we could not include these data in meta-analysis. At six months, 23 (24\%) of intervention participants were exclusively using EC and 10 (10.4\%) reported using neither EC nor combustible cigarettes (making a combined quit rate of $34.4 \%$ in the intervention arm at six months).

\section{Data from other studies}

Eight studies provided all participants with nicotine EC and assessed abstinence at six months or longer (Table $1 ; 1$ refillable, 6 cartridge, 1 not specified). The highest proportion of quitters was observed in Ely 2013 (cartridge), in which all participants (n $=48$ ) used EC and 18 used additional pharmacotherapy: 44\% of participants were abstinent at six months. The lowest quit rates were seen in two studies where participants were not motivated to quit at baseline: in Caponnetto 2013b, 14\% of participants were abstinent at 12 months, and in Polosa 2011 23\% of participants were abstinent at six months, but this fell to $13 \%$ at 24 months (both studies used cartridge devices).

\section{Adverse events}

\section{Randomized controlled trials}

Pooled data from three studies (none at high risk of bias) showed no evidence of a difference in the number of participants experiencing adverse events when comparing nicotine EC to non-nicotine EC (RR 1.01, 95\% Cl 0.91 to $1.11 ; 1^{2}=0 \% ; 601$ participants; Analysis 3.2). When comparing nicotine EC to behavioral support only or to no support, more people in the groups randomized to nicotine EC reported experiencing adverse events (RR $1.22,95 \% \mathrm{Cl} 1.12$ to 1.32 ; $\mathrm{R}^{2}=41 \%$; 4 studies, 765 participants; Analysis 4.2). As this involved unblinded comparisons with unequal levels of support, we judged all data contributing to this outcome to be at high risk of bias.

A further seven randomized controlled trials provided adverse event or related data for this comparison, but could not be included in the meta-analysis due to the way in which data were presented (see Supplemental Table 1). In the studies comparing nicotine EC to non-nicotine EC, one found similar event rates across arms (Caponnetto 2013a), and two reported more events in the nicotine EC arms (Felicione 2019; Tseng 2016). In a further study comparing nicotine to non-nicotine $\mathrm{EC}$, events were reported by type, with an increase in some seen in the nicotine group and an increase in others seen in the non-nicotine group (Lucchiari 2020). In the three studies comparing nicotine EC to behavioral support only or traditional cigarettes, Kumral 2016 found an increase in sinonasal symptoms in the group receiving nicotine EC compared to behavioral support only, and Ozga-Hess 2019 found that throat irritation, cough, and dry mouth increased in the e-cigarette group relative to the traditional cigarette group. By contrast, Pulvers 2020 found a reduction in respiratory symptoms in the e-cigarettes compared to the traditional cigarettes group.

\section{Data from other studies}

Seventeen studies provided all participants with nicotine EC and assessed adverse events at one week or longer (see Supplemental Table 1). In the seven studies which tracked event rates over time, six showed adverse events reducing over time (Bell 2017; Caponnetto 2013b; Goniewicz 2017; Polosa 2011; Polosa 2014b; Pratt 2016). Hickling 2019 showed no change. The most commonlyreported adverse events were throat/mouth irritation, headache, cough, and nausea.

\section{Serious adverse events}

\section{Randomized controlled trials}

Four studies compared nicotine EC with non-nicotine EC and reported data on SAEs; in three of these, no events occurred, so results could not contribute to the meta-analysis, although they are included in the forest plots for descriptive purposes. In the one study (Eisenberg 2020, $\mathrm{n}=255$ for this comparison) where events occurred, more were reported in the non-nicotine arm (RR 0.60, $95 \% \mathrm{Cl} 0.15$ to 2.44 ; Analysis 3.3). We rated this study at low risk of bias.

Six studies compared nicotine EC with behavioral support only or no support and reported data on SAEs; in four of these, no events occurred. Pooled results from the two studies in which events occurred showed more events occurring in the nicotine EC arm, but confidence intervals were wide and encompassed clinically significant benefit and clinically significant harm (RR 1.17, 95\% Cl 0.33 to $4.09 ;\left.\right|^{2}=5 \%$; 2 studies, 1011 participants; Analysis 4.3).

Bullen 2013, which compared nicotine EC, non-nicotine EC, and NRT, only reported that no SAEs occurred that were considered to be related to study treatment. In a study in people experiencing homelessness (Dawkins 2020), SAEs were not reported, but authors report that four to seven participants in the usual-care arm and five to seven participants in the nicotine EC arm visited Accident \& Emergency services at a hospital. Further detail can be seen in Supplemental Table 2.

\section{Data from other studies}

Seven studies provided all participants with nicotine EC and reported SAEs at a week or longer (Supplemental Table 2). In five of these (Bell 2017; Caponnetto 2013b; Humair 2014; Polosa 2011; Valentine 2018), authors report that no SAEs occurred. In NCT02648178 (19 participants), one death occurred (no further detail provided). Hickling 2019 (50 participants) recruited participants from mental health settings; five SAEs were recorded during the study, all of which were psychiatric hospitalizations. None were considered related to study treatment.

\section{Carbon monoxide}

\section{Randomized controlled trials}

Pooled data from two trials (neither considered at high risk of bias) comparing nicotine EC with non-nicotine EC found lower exhaled CO levels in people randomized to nicotine EC (MD -2.44 ppm, $95 \% \mathrm{Cl}-3.91$ to $-0.97 ; 171$ participants; Analysis 3.4). Although statistical heterogeneity was substantial $\left(I^{2}=71 \%\right)$, point estimates in both studies favored nicotine EC. Three further randomized studies measured $\mathrm{CO}$ levels in those assigned to nicotine EC and those assigned to non-nicotine EC, but did not present data in a way that could be pooled: George 2019 did not compare data by 
group; Tseng 2016 reports no between-group differences; and Meier 2017 found a slightly higher CO reading in those using nicotine EC, but the clinical and statistical significance of this difference was not clear (see Supplemental Table 3 for more detail). These data are from all study participants based on group randomized, not on subsequent $\mathrm{EC}$ or cigarette use.

Pooled data from six studies comparing nicotine EC to behavioral support alone or no support resulted in a high 12 value (92\%); pooled results are not presented here (see Analysis 4.4 for individual study data). Heterogeneity was primarily driven by magnitude rather than direction of effect, with results generally favoring nicotine EC. Three further trials reported data which could not be included in a meta-analysis. Walele 2018 compared nicotine EC to cigarettes and found CO levels declined in the EC group and remained similar to baseline in the cigarette group. Veldheer 2019 compared nicotine EC with a cigarette substitute (nonpharmacological); change in CO was similar between groups. Czoli 2019 instructed baseline dual users to spend periods only using EC or only using traditional cigarettes; $\mathrm{CO}$ measured during sole EC use was lower than baseline and lower than during cigarette-only periods. Further detail can be seen in Supplemental Table 3.

\section{Data from other studies}

Eighteen studies provided all participants with nicotine EC and reported data on $\mathrm{CO}$ at one week or longer. In the 17 studies that presented change over time, $\mathrm{CO}$ declined from baseline, although in Ikonomidis 2018 CO levels were equivalent to baseline again at 24 weeks, and in Polosa 2014b a decline was observed in people who quit smoking or reduced cigarette consumption by at least half, but not in those who continued smoking at least half as many cigarettes as they had from baseline.

\section{Heart rate}

\section{Randomized controlled trials}

One RCT (Caponnetto 2013a) provided data on heart rate and compared nicotine EC with non-nicotine EC; there was a greater decrease in heart rate in the nicotine EC arm (MD -2.80, 95\% Cl-3.85 to $-1.74 ; 141$ participants; Analysis 3.5 ). This was comparable with findings from the one RCT (Hatsukami 2020) comparing nicotine EC with no pharmacotherapy, which also found a greater reduction in the $\mathrm{EC}$ arm (MD $-2.70,95 \% \mathrm{Cl}-4.25$ to $-1.15 ; 90$ participants; Analysis 4.5).

A further three RCTs provided data on heart rate which could not be included in a meta-analysis. George 2019 compared nicotine to non-nicotine $\mathrm{EC}$ and found no difference in heart rate between arms; Walele 2018 compared a nicotine EC with a traditional cigarette and reported "no clinically significant changes", and Veldheer 2019 found decreases in both the nicotine EC and QuitSmart cigarette substitute groups, with the decrease being slightly greater in the latter group. See Supplemental Table 4 for further information.

\section{Data from other studies}

Five studies in which all participants received a nicotine EC also reported data on heart rate; changes were minimal and directions of effect were mixed (see Supplemental Table 4).

\section{Blood pressure}

Caponnetto 2013a found no difference in the change in systolic blood pressure (BP) between nicotine EC and non-nicotine EC arms (MD $0.60,95 \% \mathrm{Cl}-0.99$ to 2.19;1 41 participants; Analysis 3.6). Similarly, Hatsukami 2020 and Ikonomidis 2018 found no difference in the change in blood pressure when comparing nicotine EC to cigarettes. However, Pulvers 2020 found a benefit in favor of the EC arm; pooling data from these three studies resulted in high levels of statistical heterogeneity $\left(1^{2}=82 \%\right)$, so pooled results are not presented (Analysis 4.6). Three further RCTs measured change in blood pressure but presented results in such a way that they could not be pooled. George 2019 compared nicotine EC and non-nicotine EC and combined data from both groups; BP declined over time. Compared to a QuitSmart cigarette substitute, Veldheer 2019 found EC led to a greater reduction in BP. Walele 2018 found "no clinically significant changes" when comparing nicotine EC to a conventional cigarette at two weeks. Further data can be found in Supplemental Table 5.

Five studies which provided nicotine EC to all participants reported change in blood pressure; results were mixed and small (Hickling 2019; Ikonomidis 2018; Oncken 2015; Van Staden 2013; Walele 2018; see Supplemental Table 5).

\section{Oxygen saturation}

Hatsukami 2020 found a small increase in blood oxygen saturation when comparing nicotine EC to cigarettes (MD 0.50\%, 95\% Cl 0.31 to 0.69 ; 89 participants; Analysis 4.7). Van Staden 2013, a shortterm pre-post study which measured outcomes after two weeks of EC use, found that people who smoked who switched to ECs had significant improvement in blood oxygen saturation (96.2\% (SD 1.8) to $97.5 \%$ (SD 1.3); $1.3 \%$ increase, $95 \% \mathrm{Cl} 0.6$ to $2.1 ; \mathrm{P}=0.002$ ).

\section{Toxicants}

All randomized controlled trials measuring these outcomes compared nicotine EC with no pharmacotherapy.

Two trials measured change in 3-HPMA (one at high risk of bias). In both, the point estimate favored the EC arm, but statistical heterogeneity was substantial $\left(I^{2}=97 \%\right)$, reflecting differences in magnitude of effect. We therefore do not present a pooled result, but data from the studies can be seen in Analysis 4.8. Four further studies in which all participants were given nicotine EC measured 3-HPMA; all found reductions over time (Supplemental Table 6).

Four trials measured change in NNAL (three at high risk of bias; Analysis 4.9). Three of the four studies found results favoring nicotine EC, but for the fourth the point estimate went in the opposite direction; statistical heterogeneity was again high $\left(1^{2}=\right.$ 95\%), so pooled results are not presented. Pulvers 2018, which provided all participants with nicotine EC, found a reduction in NNAL over time, and Czoli 2019, which was a cross-over trial, found NNAL decreased when using nicotine EC compared to using traditional cigarettes (Supplemental Table 6).

One trial found reductions in 2-HPMA and AAMA compared to control (Analysis 4.10; Analysis 4.14), and a further two studies in which all participants received nicotine $\mathrm{EC}$ found reductions in both of these measures over time (Supplemental Table 6). 
One trial found reductions in S-PMA compared to control (Analysis 4.15); this was consistent with the one study (Goniewicz 2017) in which all participants received nicotine EC that measured S-PMA where levels declined over time (Supplemental Table 6).

In single trials, changes favored EC for reductions in HMPMA (Analysis 4.11), PheT (Analysis 4.12), and CEMA (Analysis 4.13). Of the 19 remaining measurements in studies where all participants received an EC, 14 reduced over time and five increased (Supplemental Table 6).

\section{Lung function}

Caponnetto 2013a measured a number of lung function parameters. FeNO increased more in the nicotine EC than the nonnicotine EC group (MD 2.35, 95\% Cl 1.78 to 2.92; 90 participants; Analysis 3.7). No difference was found between nicotine and nonnicotine EC for FEV1, FVC, or FEV1/FVC (Analysis 3.8; Analysis 3.9; Analysis 3.10).

Compared to behavioral support only/no support, Walele 2018 found improvements in FVC favoring nicotine EC (Analysis 4.16), and no difference in FEV1 or PEF 25-75 (Analysis 4.17; Analysis 4.19). Pooled data from Walele 2018 and Pulvers 2020 showed no difference in FEF 25-75, with substantial levels of statistical heterogeneity (MD $-0.06,95 \% \mathrm{Cl}-0.18$ to $0.06, \mathrm{I}^{2}=72 \% ; 2$ studies, 555 participants; Analysis 4.18).

Veldheer 2019, which randomized participants to nicotine EC or the QuitSmart cigarette substitute, measured change in a number of lung function parameters: direction of effect was mixed across these, with no statistically or clinically significant between-group differences at 12 weeks (Supplemental Table 7).

Two studies which provided all participants with nicotine EC measured change in lung function over time: Hickling 2019 found an increase in peak flow, and Oncken 2015 "no significant differences" in airway function (Supplemental Table 7).

\section{Combination therapy: nicotine EC and NRT}

This section covers two comparisons: studies in which all arms received NRT and participants were randomized to nicotine EC or non-nicotine EC, and studies in which all participants received NRT and one arm was randomized to nicotine $\mathrm{EC}$ in addition. All studies contributing data are randomized controlled trials. No studies in this group reported data on heart rate, blood pressure, oxygen, or toxicants.

\section{Cessation}

Two trials (both at high risk of bias, both testing refillable devices) in which all participants received NRT compared nicotine EC to non-nicotine EC; pooled results favored nicotine EC (RR 1.77, 95\% $\mathrm{Cl} 1.07$ to $2.94 ; \mathrm{I}^{2}=0 \% ; 1039$ participants; Analysis 5.1). Walker 2020 also compared nicotine EC + NRT to NRT alone; the point estimate favored nicotine EC but the confidence interval was wide and included no difference (Analysis 6.1).

\section{Adverse events}

The two trials (both at high risk of bias) in which nicotine ECs were compared to non-nicotine ECs in participants receiving NRT found no evidence of a difference in the number of people experiencing AEs between arms; data from Walker 2020 can be seen in Analysis 5.2; Baldassarri 2018 reported results combined across groups but noted "no significant differences by treatment group" (Supplemental Table 1).

The two trials comparing nicotine EC + NRT to NRT alone that contributed data to this outcome were both at high risk of bias. Statistical heterogeneity was high when combining data $(12=79 \%)$ and hence we do not present pooled results. In one study (Walker 2020), AEs were lower in the EC group and the confidence interval excluded no difference, while in the other study (Guillaumier 2018) AEs were higher in the EC group but the confidence interval was wide (Analysis 6.2).

\section{Serious adverse events}

Walker 2020, comparing nicotine EC with non-nicotine EC as adjuncts to NRT, had fewer SAEs in the nicotine EC group than in the non-nicotine EC group, but the confidence interval includes no difference (Analysis 5.3).

Three studies provided data on SAEs and compared nicotine EC + NRT to NRT alone. The pooled estimate favored the NRT-alone group, but again the confidence interval was wide and included no difference (RR $1.26,95 \% \mathrm{Cl} 0.46$ to $3.42: \mathrm{I}^{2}=0 ; 682$ participants; Analysis 6.3).

\section{Carbon monoxide}

Walker 2020 (which compared nicotine EC + NRT, non-nicotine $\mathrm{EC}+\mathrm{NRT}$, and NRT alone) measured change in CO levels but did not report data in a way that could be pooled. $\mathrm{CO}$ declined over time, with the greatest reduction seen in the nicotine EC group (see Supplemental Table 3). Baldassarri 2018, comparing nicotine and non-nicotine EC as adjuncts to NRT, found a slightly greater reduction in $\mathrm{CO}$ in the nicotine $\mathrm{EC}$ group, but the confidence interval included no clear evidence of a difference (Analysis 5.4) between groups.

\section{Lung function}

Baldassarri 2018, which compared nicotine EC to non-nicotine EC and in which both groups received NRT, found no between-group differences in FeNO, FEV1, or FVC (Analysis 5.5; Analysis 5.6; Analysis 5.7); confidence intervals were wide for all outcomes.

\section{Comparisons based on nicotine dose}

Two trials provided data comparing different doses of nicotine in EC (although other studies provided a range of doses, these were not randomly assigned). In Caponnetto 2013a, where different concentrations were available via the same device, cessation and adverse event data were not available. No serious adverse events were reported in either arm (Analysis 7.1). There were no clinical or statistically significant differences between arms for carbon monoxide, heart rate, blood pressure, or lung function measures (Analysis 7.2 to Analysis 7.8). In Yingst 2020 (cross-over, comparing different doses and different devices) exhaled $\mathrm{CO}$ and reported nausea were not different between devices; self-reported dizziness was low overall but slightly higher in the higher-dose arm. Further detail can be found in Supplemental Table 1 and Supplemental Table 3. 


\section{Non-nicotine EC}

Although non-nicotine ECs serve as a 'control group' in our primary analysis, due to their behavioral properties they can also be considered an intervention in and of themselves. Comparisons included here are: non-nicotine EC versus NRT; non-nicotine EC versus usual care; and non-nicotine EC as an adjunct to NRT. All contributing data are from randomized controlled trials. None of these studies reported data on change in $\mathrm{CO}$, heart rate, blood pressure, oxygen saturation, toxicants, or lung function.

\section{Cessation}

When comparing non-nicotine EC to behavioral support only, pooled results from two studies $(n=388)$ found higher quit rates in participants randomized to non-nicotine $\mathrm{EC}$, but the confidence interval included the possibility of no difference (RR $1.74,95 \% \mathrm{Cl}$ 0.76 to $3.96 ; 1^{2}=0 \%$; Analysis 8.1 ). When evaluating non-nicotine EC as an adjunct to NRT, Walker 2020 also found higher quit rates in participants randomized to non-nicotine EC, although again the confidence interval included no difference (Analysis 9.1).

Lee 2019 compared non-nicotine EC with NRT; the point estimate favored NRT but the confidence interval included no difference (Analysis 10.1).

\section{Adverse events}

Eisenberg 2020 found a higher rate of adverse events in the EC arm than in behavioral support only, with the confidence interval excluding no difference (Analysis 8.2). By contrast, Walker 2020 found fewer adverse events in participants receiving non-nicotine EC + NRT compared to NRT alone, with the confidence interval excluding no difference (Analysis 9.2). Lee 2019 also found that fewer participants receiving non-nicotine EC reported adverse events than those receiving NRT, with the confidence interval excluding no difference (Analysis 10.2).

\section{Serious adverse events}

Eisenberg 2020 found a higher rate of SAEs in the EC arm than in the behavioral support-only arm, but confidence intervals were wide and incorporated clinically significant benefit and clinically significant harm (Analysis 8.3). In Walker 2020, more SAEs occurred in the group randomized to non-nicotine EC + NRT than in the NRTalone group, but the confidence interval included no difference as well as the potential for a clinically significant difference in favor of the intervention (Analysis 9.3). No SAEs were reported in either arm of Lee 2019 (non-nicotine EC versus NRT).

\section{DISCUSSION}

\section{Summary of main results}

This update includes a further six studies compared with the previously published version. Our three main comparisons, nicotine EC compared to NRT, nicotine EC compared to nonnicotine EC, and nicotine EC compared to behavioral support only/ no support still show increased quit rates in people assigned to nicotine EC arms; this is moderate-certainty for the first two comparisons, and very low certainty for the latter (Summary of findings 1; Summary of findings 2; Summary of findings 3 ). In absolute terms, pooled data suggest an additional four people for every 100 would quit smoking with nicotine EC compared to nonnicotine EC or to NRT, and that an additional seven people per
100 would quit if offered a nicotine EC compared to being offered behavioral support alone or no support. Most data come from studies of cartridge devices which deliver relatively little nicotine in comparison to newer device models. However, within newer device models with better nicotine delivery, this update includes the first included study of a pod device (Pulvers 2020).

The certainty of the evidence for adverse events in nicotine EC compared to non-nicotine EC has been upgraded from low- to moderate-certainty evidence of no difference. Evidence on adverse events (AEs) and serious adverse events (SAEs) was of low to very low certainty across all other comparisons, due to a paucity of data. SAEs were rare, in both intervention and comparator arms, with many of the studies which measured SAEs reporting no such events in either study arm. For nicotine EC compared to non-nicotine $E C$, pooled data suggest no difference in the number of people experiencing AEs and one fewer person per 100 experiencing SAEs with nicotine EC compared to non-nicotine EC arms, but confidence intervals include no difference. Conversely, data from comparisons between nicotine EC and behavioral support alone or no support suggest an additional 13 people per 100 assigned to nicotine EC may experience $A E s$, with no difference in the number experiencing SAEs. As with AEs from other smoking cessation treatments (e.g. NRT, Hartmann-Boyce 2018a), these events typically related to irritation at site (e.g. dry mouth, cough) and resolved over time. Compared to NRT, one fewer person per 100 might be expected to experience an $\mathrm{AE}$ if assigned to nicotine $\mathrm{EC}$, and two additional people per 100 might be expected to experience an SAE. These figures should be treated with caution, due to large confidence intervals encompassing no clinically significant difference. The small amount of contributing data, and the variation in 'control group' risk across comparisons, reflect different methods of collecting data and different lengths of follow-up. No studies in any of the different comparison conditions detected serious harms considered to be related to EC use.

Beyond AEs and SAEs, we consider data on a range of safety- and health-related outcomes, including carbon monoxide and other toxins, lung function, blood pressure, pulse, and oxygen levels. Data on all of these outcome measures are limited; for most outcomes within most comparisons, only one study currently contributes data. Pooled data from two studies in which all participants received nicotine replacement therapy showed that nicotine EC led to higher quit rates than non-nicotine EC, but we judged both studies to be at high risk of bias, meaning the effect remains uncertain.

\section{Overall completeness and applicability of evidence}

This field of research and EC devices themselves continue to evolve rapidly. This is the first update conducted as part of our 'living systematic review' approach, with which we will proceed for at least the next 12 months, meaning we can continue to rapidly incorporate new evidence (see Appendix 1). This is important, as all of our analyses currently suffer from imprecision.

This update captures data from the past year, up to February 2021. Subsequent monthly searches will keep this review current. Although studies predominantly came from the USA and UK, overall this review covers data from 14 countries; geographical range in studies may be particularly important in this area, due to the marked differences in EC regulation between countries; for example, studies conducted in countries that limit nicotine 
dose in EC or allow only certain EC devices to be tested may observe less pronounced effects on quitting. This review includes studies in some 'harder to reach' populations, including people not motivated to quit smoking, people with substance misuse disorders, and people experiencing homelessness. Quit rates in these groups are traditionally lower, which may make it more difficult to detect effects of interventions. However, it could be that these groups may particularly stand to benefit from EC if they are effective, because in absolute terms conventional cessation methods are often not as effective for them.

As well as the rapid pace of research in this field, EC technology itself continues to evolve, which poses a challenge when considering the applicability of our evidence to the present. We had downgraded the certainty of our data in the 2016 update, as the devices tested in the trials were first-generation 'cig-a-like' devices which did not deliver nicotine well, meaning the studies may have yielded more conservative estimates than would be seen with newer models, as newer devices and models have tended towards improved nicotine delivery. Nicotine delivery is also relevant to the comparator NRT arms tested; use of both a shorter- and a longeracting form of NRT shows the highest success, and it is important that where possible this be the comparator chosen for such trials (Lindson 2019).

Regarding EC device type, in the 2020 update and now in this update, we have more data from newer devices, although there will always be a time lag between current devices and the research evidence available. None of the analyses of our primary outcomes signified substantial levels of statistical heterogeneity, despite the fact that different devices were used in the included studies. However, this could be because confidence intervals were wide for individual studies, and does not rule out clinically significant differences in effects between EC types. As further data emerge, we hope to be able to formally test for differences in subgroup analyses, and ideally over time in head-to-head comparisons of different device types. Our review now includes one study of a pod device (Pulvers 2020) and one study directly comparing device types (Yingst 2020). However, neither contributes data to our cessation outcomes.

The adverse effects described in both the RCT and cohort studies continue to look similar, regardless of the brand of EC used or nicotine content, with placebo and nicotine-containing ECS showing similar numbers and types of adverse events in direct comparisons. They also reflect what is reported in survey data (Dawkins 2013b; Etter 2011), so we believe that they are broadly applicable to most EC brands.

There has been concern raised that the dual use of cigarettes and EC may expose people to greater health risks, including higher nicotine levels. However, given that people who smoke like to maintain relatively stable blood nicotine levels (Russell 1990), receiving nicotine from an alternative source (i.e. EC) is likely to reduce nicotine intake from cigarettes, which should be accompanied by a reduction in smoke and toxin intake (Fagerström 2004). In a study assessing biochemical changes exclusively in dual-users, there was a significant decrease in exhaled carbon monoxide levels and urinary 3-HMPA (McRobbie 2015). In this update, we found one new study conducted in dual-users. Similar to McRobbie 2015, Czoli 2019 found that levels of biomarkers of exposure to toxicants were significantly lower when participants exclusively used EC compared to dual use; by contrast, biomarkers of exposure increased when participants exclusively smoked as compared to dual use. These results are supported by longer-term studies in people who smoke and were provided with ECs, which found decreases in exhaled carbon monoxide among dual-users (Adriaens 2014; Pacifici 2015; Polosa 2011; Polosa 2014b).

The structure of our analyses follows standard practice of the Cochrane Tobacco Addiction Group, i.e. evaluating outcomes on an intention-to-treat basis, meaning our pooled results represent the effect of offering an EC intervention. This is different from evaluating the per protocol effect, or the effect only in those who use the EC to quit smoking entirely, or continue to smoke whilst also using EC. Some of our included studies have also assessed data using these groupings and we have attempted to note this in the supplemental tables. Although pragmatic and hopefully of use to those designing and delivering interventions, we acknowledge that our intentionto-treat approach limits the ability to use the data presented here to draw conclusions about biomarkers in subgroups of participants based on subsequent EC use/smoking profiles.

\section{Certainty of the evidence}

We consider the certainty of the evidence below as it relates to primary outcomes for our three main comparisons: nicotine EC versus NRT; nicotine EC versus non-nicotine EC; nicotine EC versus behavioral support only/no support (Summary of findings 1; Summary of findings 2; Summary of findings 3). The certainty of evidence for all other comparisons and outcomes should be considered very low due to a paucity of data.

Our 'Summary of findings' tables and assessments of certainty are based on the evidence from randomized controlled trials (RCTs). The cohort studies that we include were all deemed to have high risks of bias, which is inherent in the study design. Data presented from these studies need to be interpreted with caution. However, data from cohort studies were reassuringly consistent with data from RCTs.

Risk of bias did not impact on the certainty of evidence for comparisons between nicotine and non-nicotine EC, or between nicotine EC and NRT. For the latter, we judged all three studies to be at low risk of bias overall. For the former, removing the one study at high risk of bias increased the effect estimate for our efficacy outcome. Risk of bias decreased our certainty in the effect estimates for our nicotine EC versus behavioral support only/ no support comparison, as due to the nature of the comparison, blinding was not possible and differential levels of support could lead to bias. All but one of our main comparisons were downgraded for imprecision, due to wide confidence intervals and few events. Other than risk of bias and imprecision, we identified no other issues which decreased the certainty of the primary outcomes for our main comparisons. In the previous version of this review we had downgraded cessation outcomes for indirectness, due to the included studies testing devices that were no longer available due to poor nicotine delivery (we therefore judged it plausible that our analyses could be underestimating the effect of devices available at the time the review was published). In this version, we no longer downgrade on this basis, as this update includes a wider range of EC models, including more recent devices, and heterogeneity in outcomes remains low. 


\section{Cessation}

All three comparisons found effect estimates favoring nicotine EC for smoking cessation. For nicotine EC versus non-nicotine EC and for nicotine EC versus NRT, we judged the evidence to be of moderate certainty, meaning we think the true effect is likely to be close to the estimate of effect. For nicotine EC versus behavioral support only/no support, we judged the evidence to be of very low certainty, meaning we have very little confidence in the effect estimate. Another way to look at this, however, is to consider that nicotine $\mathrm{EC}$ versus non-nicotine $\mathrm{EC}$ comparisons isolate the effect of nicotine as provided by an EC, and nicotine EC versus NRT comparisons isolate the effect of the sensorimotor elements provided by an EC. Given that both of these comparisons find a benefit of nicotine EC for smoking cessation, it might logically follow that the comparison between nicotine EC and behavioral support only/no support would find a benefit in favor of nicotine $\mathrm{EC}$, since this comparison would capture both pharmacological and sensorimotor mechanisms of effect. This increases our confidence in the effect of nicotine EC when compared to behavioral support alone or to no support.

\section{Adverse and serious adverse events}

In this update, we have upgraded the certainty of evidence for adverse events in the nicotine EC versus non-nicotine EC comparison; the addition of new data has led to narrower confidence intervals, and as a result we have upgraded the evidence from low to moderate certainty of no difference. For our other comparisons and adverse effect outcomes, effect estimates of adverse events and serious adverse events were judged to be of low or very low certainty, with the main problem being imprecision. This means the true effect may be substantially different from the estimate of the effect. None of the analyses signaled serious harm, nor did complementary data from cohort studies, but unlike our cessation analyses, many of the confidence intervals encompassed the possibility of both clinically significant harm and clinically significant benefit. This uncertainty should reduce as more studies become available.

\section{Potential biases in the review process}

We consider the review process we used to be robust. For outcome assessment, we followed the standard methods used for Cochrane Tobacco Addiction Review Group cessation reviews. Our search strategy included the Cochrane Tobacco Addiction Group Specialized Register and we were able to capture a number of ongoing studies. However, there may be unpublished data that our searches did not uncover. We also considered participants lost to follow-up as continuing to smoke, which is standard practice in this field. There are concerns that frequently updating meta-analyses can lead to issues with multiple testing; we followed Cochrane guidance in conducting this living systematic review and hence do not adjust for multiple testing (Brooker 2019).

Three of our review authors are authors of included studies. These authors were not involved in the decisions about inclusion of their studies, or in data extraction or 'Risk of bias' assessment for these studies.

\section{Agreements and disagreements with other studies or reviews}

This Cochrane Review aligns with but updates the conclusions of the 2018 U.S. National Academies of Science, Engineering, and Medicine's Consensus Study Report, Public Health Consequences of E-cigarettes (NASEM 2018), which reviewed literature published through August 2017 to address the question, "Do e-cigarettes help smokers quit smoking combustible tobacco cigarettes?". Focusing on RCTs and existing systematic reviews, it used a prespecified Level of Evidence framework to develop conclusions. The report's overall conclusion was that there was "limited evidence that ecigarettes may be effective aids to promote smoking cessation." Based on the RCTs available, it concluded that there was "moderate evidence" that e-cigarettes containing nicotine were more effective for cessation than e-cigarettes without nicotine, but "insufficient evidence" about the effectiveness of e-cigarettes compared to no treatment or to FDA-approved smoking cessation treatments. Our review contradicts this latter point, as we now find moderatecertainty evidence of benefit when comparing nicotine EC with NRT; this is primarily due to a large RCT published after NASEM 2018. A 2021 review from Public Health England, which cites the 2020 version of this Cochrane Review, concludes that, compared to their 2018 review, there is now stronger evidence that nicotine vaping products are effective for smoking cessation (McNeill 2021).

Findings are also broadly consistent with those from other reviews published in the past year, with some exceptions. Amato 2020 did not evaluate effectiveness and focused only on safety; consistent with our review, they found very low- to moderate-certainty evidence on a range of possible adverse effects, with the most frequently reported being cough, dry mouth, shortness of breath, irritation of the mouth and throat, and headache. Akiyama 2021 reviewed biomarker findings from clinical studies and concluded that the use of EC could lead to a significant reduction in exposure to harmful substances compared to traditional cigarettes; this is again consistent with findings from our review. Martinez-Morata 2021 reviewed blood pressure findings and concluded that EC may result in short-term elevations, but that more data are needed; our review also lacks sufficient data to draw any conclusions about blood pressure at one week or longer.

Zhang 2021 conducted a rapid review; while their pooled analysis also suggested that EC increased quit rates compared to NRT or non-nicotine EC, they judged the evidence to be of low certainty according to GRADE. As with us, they downgraded by one level due to inconsistency, but unlike us they also downgraded by one level for statistical heterogeneity. Zhang 2021 combined studies with NRT comparators and those with non-nicotine EC comparators in the same analysis and found moderate statistical heterogeneity; we evaluated these two comparisons separately and did not find evidence of statistical heterogeneity. Patnode 2021 reviewed evidence on tobacco cessation interventions for the US Preventive Services Task Force (USPFTS 2021). They state that none of their included EC trials suggested higher rates of serious adverse events; this is in line with our analyses. However, they report that findings across EC trials were inconsistent for effectiveness, with some finding statistically significant evidence of benefit and some finding no statistically significant difference. They did not conduct statistical meta-analyses and include five trials, all of which are included in our cessation meta-analyses. None of our cessation meta-analyses, which include these trials, detected levels 
of heterogeneity beyond what would be expected from chance alone. Wang 2021 reviewed data both from observational studies and from randomized controlled trials; in the trials, e-cigarettes were associated with increased smoking cessation (as with our review). In observational studies, ECs were not associated with increased smoking cessation. As discussed in Methods, although we included non-randomized studies in which an EC intervention is provided in this review, we do not include observational studies in which no EC intervention is provided, due to known issues with confounding.

Reviews of ECs for policymaking are often broader in scope than our review, which focuses exclusively on their role in supporting smoking cessation in people who smoke. Outside of smoking cessation, there remain unanswered questions about the impact of EC availability and use on young people; we hope to evaluate this in a separate review.

\section{AUTHORS' CONCLUSIONS}

\section{Implications for practice}

Evidence suggesting nicotine EC can aid in smoking cessation is consistent across several comparisons. There was moderatecertainty evidence, limited by imprecision, that EC with nicotine increased quit rates at six months or longer compared to nonnicotine EC and compared to NRT. There was very low-certainty evidence (limited by risk of bias as well as imprecision) that EC with nicotine increased quit rates compared to behavioral support alone or to no support.

\section{The effect of nicotine EC when added to NRT was unclear.}

None of the included studies (short- to mid-term, up to two years) detected serious adverse events considered possibly related to $\mathrm{EC}$ use. The most commonly-reported adverse effects were throat/ mouth irritation, headache, cough, and nausea, which tended to dissipate with continued use. In some studies, reduced toxin concentrations and biomarkers of harm were observed in people who smoked and switched to vaping, consistent with reductions seen in smoking cessation.

\section{Implications for research}

Further randomized controlled trials of nicotine EC are needed, following up participants at six months or longer. Studies with active comparators (i.e. comparing nicotine EC to frontline smoking cessation pharmacotherapies) are likely to be of particular use to decision-makers. All studies (including uncontrolled intervention cohort studies) should aim to assess the safety profile of electronic cigarettes for as long as possible (the current review only includes data up to two years), and ideally be powered to detect differences in safety outcomes, including adverse events and serious adverse events. Evidence from one well-conducted RCT suggests that people who quit smoking using EC may continue to use EC longer than they might use other stop-smoking pharmacotherapies, making assessments of their long-term safety profile particularly important. Safety results should be presented in both absolute and relative risk terms (in comparison to the risks of continuing to smoke tobacco).

Studies should offer recent devices to participants, to be most representative of what will be on the market at the time results are released. Data on pod-type EC are particularly lacking, though we now include one trial. Protocols and statistical analysis plans should be registered in advance and openly available.

Further RCTs need to be adequately powered. Further trials of pod devices would be of particular value, as would RCTs providing EC in a way that would be used in real-world settings (e.g. taking into account individual preferences for strengths and flavors of e-liquids and even EC devices, and also allowing for changes in preferences over time).

Further reviews, using best available methods, need to be conducted to evaluate the possible relationships between EC use and availability and youth uptake of EC and conventional cigarettes.

\section{ACKNOWLEDGEMENTS}

The 2020 update was supported by the Cochrane Incentives Award Scheme and the University of Oxford Returning Carer's Fund, as well as through core infrastructure funding from the National Institute for Health Research (NIHR) for the Cochrane Tobacco Addiction Review Group. This update and the 2020 update have also been supported through a Tobacco Advisory Group Cancer Research UK Project Grant. The views and opinions expressed therein are those of the authors and do not necessarily reflect those of the Systematic Reviews Programme, NIHR, National Health Service (NHS) or the Department of Health.

We thank Dr Jonathan Livingstone-Banks for running monthly searches.

We would like to thank Assistant Professor Stephen R. Baldassarri, Drs Pasquale Caponnetto, Fabio Cibella and Professor Riccardo Polosa, Professor Matthew Carpenter, Bruce R. Lindgren and Professor Dorothy Hatsukami, Assistant Professor Tracy Smith, Assistant Professor Susan Veldheer, Lauren Hickling, Stephanie K. Bell, Associate Professor Coral Gartner, Professor Billie Bonevski, Karolien Adriaens, Dr Sharon Cox, Professor Lynne E. Dawkins, Professor Mark Eisenberg, Andrea Hebert-Losier, Kris Filion, Dr Ignatios Ikonomidis, Dr Kim Pulvers, Florian Scheibein, Dr Markos Klonizakis, Dr Scott Sherman, Dr Kylie Morphett, Dr Ivan Berlin, Marzena Orzol, Dr Katie Myers Smith and Christine Czoli for providing additional data or information. We would like to thank Dr Sharon Cox and Professor Lynne E. Dawkins for performing peer review for this update, and Dr Debbie Robson for performing editorial review for this update. 


\section{R E F E R E N C E S}

\section{References to studies included in this review}

Adriaens 2014 \{published data only\}

Adriaens K, Van Gucht D, Declerck P, Baeyens F. Effectiveness of the electronic cigarette: an eight-week Flemish study with six-month follow-up on smoking reduction, craving and experienced benefits and complaints. International Journal of Environmental Research and Public Health 2014;11(11):11220-48.

\section{Baldassarri 2018 \{published data only\}}

* Baldassarri SR, Bernstein SL, Chupp GL, Slade MD, Fucito LM, Toll BA. Electronic cigarettes for adults with tobacco dependence enrolled in a tobacco treatment program: a pilot study. Addictive Behaviors May 2018;80:1-5.

NCT02498145. Short term effects of electronic cigarettes in tobacco dependent adults. clinicaltrials.gov/show/ NCT02498145 (accessed 17 February 2016).

\section{Bell 2017 \{unpublished data only\}}

ACTRN12616001641482. Tobacco harm reduction with vaporised nicotine (THRiVe): feasibility study. www.anzctr.org.au/Trial/Registration/TrialReview.aspx? ACTRN=12616001641482 (first received 28 November 2016).

Bell S, Dean J, Gilks C, Boyd MA, Fitzgerald L, Mutch A, et al. Tobacco harm reduction with vaporised nicotine (THRiVe): the study protocol of an uncontrolled feasibility study of novel nicotine replacement products among people living with HIV who smoke. International Journal of Environmental Research and Public Health 2017;14(7):799.

\section{Bullen 2013 \{published data only\}}

Bullen C, Howe C, Laugesen M, McRobbie H, Parag V, Williman J, et al. Electronic cigarettes and smoking cessation: a quandary? Authors' reply. Lancet 2014;383(9915):408-9.

* Bullen C, Howe C, Laugesen M, McRobbie H, Parag V, Williman J, et al. Electronic cigarettes for smoking cessation: a randomised controlled trial. Lancet 2013;382(9905):1629-37.

Bullen C, Howe C, Laugesen M, McRobbie H, Parag V, Williman J. Do electronic cigarettes help smokers quit? Results from a randomised controlled trial [Abstract]. In: European Respiratory Society Annual Congress, 2013 September 7 - 11, Barcelona, Spain. Vol. 42. 2013:215s-[P1047].

Bullen C, Williman J, Howe C, Laugesen M, McRobbie H, Parag V, et al. Study protocol for a randomised controlled trial of electronic cigarettes versus nicotine patch for smoking cessation. BMC Public Health 2013;13:210.

O'Brien B, Knight-West O, Walker N, Parag V, Bullen C. Ecigarettes versus NRT for smoking reduction or cessation in people with mental illness: secondary analysis of data from the ASCEND trial. Tobacco Induced Diseases 2015;13(1):5.

\section{Caponnetto 2013a \{published data only\}}

Campagna D, Cibella F, Caponnetto P, Amaradio MD, Caruso M, Morjaria JB, et al. Changes in breathomics from a 1-year randomized smoking cessation trial of electronic cigarettes. European Journal of Clinical Investigation 2016;46(8):698-706.

* Caponnetto P, Campagna D, Cibella F, Morjaria JB, Caruso M, Russo C, et al. EffiCiency and Safety of an eLectronic cigAreTte (ECLAT) as tobacco cigarettes substitute: a prospective 12 -month randomized control design study. PlOS One 2013;8(6):e66317.

Cibella F, Campagna D, Caponnetto P, Amaradio MD, Caruso M, Russo $C$, et al. Lung function and respiratory symptoms in a randomized smoking cessation trial of electronic cigarettes. Clinical Science 2016;130(21):1929-37.

Farsalinos K, Cibella F, Caponnetto P, Campagna D, Morjaria JB, Battaglia $E$, et al. Effect of continuous smoking reduction and abstinence on blood pressure and heart rate in smokers switching to electronic cigarettes. Internal and Emergency Medicine 2016;11(1):85-94.

NCT01164072. Efficacy and safety of an electronic nicotine delivery device (e-cigarette). clinicaltrials.gov/ct2/show/ NCT01164072 (first received 16 July 2010).

NCT01194583. Efficacy and safety of an electronic nicotine delivery device (e-cigarette) without nicotine cartridges. clinicaltrials.gov/ct2/show/NCT01194583 (first received 3 September 2010).

Russo C, Cibella F, Caponnetto P, Campagna D, Maglia M, Frazzetto $\mathrm{E}$, et al. Evaluation of post cessation weight gain in a 1-year randomized smoking cessation trial of electronic cigarettes. Scientific Reports 2016;6:18763.

Caponnetto 2013b \{published data only\}

* Caponnetto P, Auditore R, Russo C, Cappello GC, Polosa R. Impact of an electronic cigarette on smoking reduction and cessation in schizophrenic smokers: a prospective 12-month pilot study. International Journal of Environmental Research and Public Health 2013;10(2):446-61.

Minutolo G, Caponnetto P, Auditore R, Russo C, Polosa R. Management of smoking reduction and cessation in inpatients with schizophrenia: Impact of electronic cigarettes. European Neuropsychopharmacology 2013;23:S581-2.

\section{Carpenter 2017 \{published data only\}}

* Carpenter MJ, Heckman BW, Wahlquist AE, Wagener TL, Goniewicz ML, Gray KM, et al. A naturalistic, randomized pilot trial of e-cigarettes: uptake, exposure, and behavioral effects. Cancer Epidemiology, Biomarkers \& Prevention 2017;26(12):1795-803.

NCT02357173. A trial of e-cigarettes: natural uptake, patterns and impact of use. clinicaltrials.gov/show/NCT02357173 (accessed 17 February 2016).

Smith TT, Wahlquist AE, Heckman BW, Cummings KM, Carpenter MJ. Impact of e-cigarette sampling on cigarette dependence and reinforcement value. Nicotine \& Tobacco Research 2020;22(2):297-301. 
Czoli 2019 \{published data only\}

Czoli CD, Fong GT, Goniewicz ML, Hammond D. Biomarkers of exposure among "dual users" of tobacco cigarettes and electronic cigarettes in Canada. Nicotine and Tobacco Research 2019;21(9):1259-66.

Dawkins 2020 \{published and unpublished data\}

* Dawkins L, Bauld L, Ford A, Robson D, Hajek P, Parrott S, et al. A cluster feasibility trial to explore the uptake and use of e-cigarettes versus usual care offered to smokers attending homeless centres in Great Britain. PLOS One 2020;15(10). [ISRCTN14140672]

ISRCTN14140672. Exploring the use and uptake of e-cigarettes for homeless smokers. www.isrctn.com/ISRCTN14140672 (first received 7 November 2018).

\section{Eisenberg 2020 \{published data only\}}

* Eisenberg MJ, Hébert-Losier M, Windle SB, Greenspoon T, Brandys T, Fülöp T. Effect of e-cigarettes plus counseling vs counseling alone on smoking cessation: a randomized clinical trial. JAMA 2020;324(18):1844-54.

Hebert-Losier A, Filion KB, Windle SB, Eisenberg MJ. A randomized controlled trial evaluating the efficacy of ecigarette use for smoking cessation in the general population: E3 trial design. Canadian Journal of Cardiology Open 2020;2(3):168-75. [NCT02417467]

NCT02417467. Evaluating the efficacy of e-cigarette use for smoking cessation (E3) trial. clinicaltrials.gov/show/ NCT02417467 (accessed 17 February 2016).

\section{Ely 2013 \{published data only\}}

Ely J. Evaluation of the use of electric cigarettes in a rural smoking cessation program. Available online at: digitalunc.coalliance.org/fedora/repository/cogru:4161 [no longer available] 2013 (accessed 1st November 2014).

\section{Felicione 2019 \{published data only\}}

Felicione NJ, Enlow P, Elswick D, Long D, Sullivan CR, Blank MD. A pilot investigation of the effect of electronic cigarettes on smoking behavior among opioid-dependent smokers. Addictive Behaviors 2019;91:45-50.

\section{George 2019 \{published data only\}}

* George J, Hussain M, Vadiveloo T, Ireland S, Hopkinson P, Struthers AD, et al. Cardiovascular effects of switching from tobacco cigarettes to electronic cigarettes. Journal of the American College of Cardiology 2019;74(25):3112-20.

NCT02878421. Vascular EffectS of regUlar cigarettes Versus electronlc cigarette USe (VESUVIUS). clinicaltrials.gov/ct2/ show/NCT02878421 (first received 25 August 2016).

\section{Goniewicz 2017 \{published data only\}}

* Goniewicz ML, Gawron M, Smith DM, Peng M, Jacob P 3rd, Benowitz NL. Exposure to nicotine and selected toxicants in cigarette smokers who switched to electronic cigarettes: a longitudinal within-subjects observational study. Nicotine \& Tobacco Research 2017;19(2):160-7.
Guillaumier 2018 \{unpublished data only\}

ACTRN12617000849392. The QuitNic Study: a pilot study of electronic nicotine devices for smoking cessation with drug and alcohol clients. www.anzctr.org.au/Trial/Registration/ TrialReview.aspx?id=372198 (first received 8 June 2017).

Bonevski B, Manning V, Wynne O, Gartner C, Borland R, Baker A, et al. QuitNic: A pilot randomised controlled trial comparing nicotine vaping products with nicotine replacement therapy for smoking cessation following residential detoxification. Nicotine and Tobacco Research 2021;23(3):462-70.

* Guillaumier A, Manning V, Wynne O, Gartner C, Borland R, Baker AL, et al. Electronic nicotine devices to aid smoking cessation by alcohol- and drug-dependent clients: protocol for a pilot randomised controlled trial. Trials 2018;19:415.

Hajek 2015a \{published data only\}

Hajek P, Corbin L, Ladmore D, Spearing E. Adding e-cigarettes to specialist stop-smoking treatment: City of London pilot project. Journal of Addiction Research \& Therapy 2015;6(244):(online ahead of print). [DOI: 10.4172/2155-6105.1000244]

Hajek 2019 \{published and unpublished data\}

* Hajek P, Phillips-Waller A, Przulj D, Pesola F, Myers Smith K, Bisal N, et al. A randomized trial of e-cigarettes versus nicotinereplacement therapy. New England Journal of Medicine 2019;380(7):629-37.

Hajek P, Phillips-Waller A, Przulj D, Pesola F, Smith KM, Bisal N, et al. E-cigarettes compared with nicotine replacement therapy within the UK Stop Smoking Services: the TEC RCT. Health Technology Assessment 2019;23(43):1-82. [ISRCTN60477608]

ISRCTN60477608. The efficacy of e-cigarettes compared with nicotine replacement therapy, when used within the UK stop smoking service. www.isrctn.com/ISRCTN60477608 2014 (first received 2 April 2015).

Li J, Hajek P, Pesola F, Wu Q, Phillips-Waller A, Przulj D, et al. Cost-effectiveness of e-cigarettes compared with nicotine replacement therapy in stop smoking services in England (TEC study): a randomized controlled trial. Addiction 2020;115(3):507-17.

\section{Halpern 2018 \{published data only\}}

* Halpern SD, Harhay MO, Saulsgiver K, Brophy C, Troxel AB, Volpp KG. A pragmatic trial of e-cigarettes, incentives, and drugs for smoking cessation. New England Journal of Medicine 2018;378(24):2302-10.

NCT02328794. Randomized clinical trial to reduce harm from tobacco. clinicaltrials.gov/show/NCT02357173 (accessed 17 February 2016).

\section{Hatsukami 2020 \{published and unpublished data\}}

Hatsukami D, Meier E, Lindgren BR, Anderson A, Reisinger S, Norton $\mathrm{K}$, et al. A randomized clinical trial examining the effects of instructions for electronic cigarette use on smoking-related behaviors, and biomarkers of exposure. Nicotine \& Tobacco Research 2020;22(9):1524-32. [DOI: 10.1093/ntr/ntz233] 
Hickling 2019 \{published data only\}

* Hickling LM, Perez-Iglesias R, McNeill A, Dawkins L, Moxham J, Ruffell T, et al. A pre-post pilot study of electronic cigarettes to reduce smoking in people with severe mental illness. Psychological Medicine 2019;49(6):1033-40.

NCT02212041. Acceptability, patterns of use and safety of electronic cigarette in people with mental illness: a pilot study. clinicaltrials.gov/show/NCT02212041 (accessed 17 February 2016).

\section{Holliday 2019 \{published data only\}}

* Holliday R, Preshaw PM, Ryan V, Sniehotta FF, McDonald S, Bauld L, et al. A feasibility study with embedded pilot randomised controlled trial and process evaluation of electronic cigarettes for smoking cessation in patients with periodontitis. Pilot and Feasibility Studies 2019;5:74.

ISRCTN17731903. Feasibility study of e-cigarettes in periodontitis. www.isrctn.com/ISRCTN17731903 (first received 27 September 2016).

\section{Humair 2014 \{published data only\}}

Humair J-P, Tango R. Can e-cigarette help patients to reduce or stop smoking in primary care practice? Journal of General Internal Medicine 2014;29:S480.

\section{Ikonomidis 2018 \{published data only\}}

* Ikonomidis I, Vlastos D, Kourea K, Kostelli G, Varoudi M, Pavlidis $\mathrm{G}$, et al. Electronic cigarette smoking increases arterial stiffness and oxidative stress to a lesser extent than a single conventional cigarette: an acute and chronic study. Circulation 2018;137(3):303-6

NCT03039920. The effects of electronic cigarette smoking on the arterial wall and endothelial glycocalyx properties of smokers. clinicaltrials.gov/ct2/show/NCT03039920 (first received 1 February 2017).

\section{Ikonomidis 2020 \{published data only\}}

Ikonomidis I, Katogiannis K, Kostelli G, Kourea K, Kyriakou E, Kypraiou A, et al. Effects of electronic cigarette on platelet and vascular function after four months of use. Food and Chemical Toxicology 2020;141:111389.

\section{loakeimidis 2018 \{published data only\}}

loakeimidis N, Vlachopoulos C, Georgakopoulos C, Abdelrasoul M, Skliros N, Katsi V, et al. Smoking cessation rates with varenicline and electronic cigarettes in relapsed smokers with a history of acute coronary syndrome. European Heart Journal 2018;39(Suppl_1):242.

Kumral 2016 \{published data only\}

Kumral TL, Saltürk Z, Yildirim G, Uyar Y, Berkiten G, Atar Y, et al. How does electronic cigarette smoking affect sinonasal symptoms and nasal mucociliary clearance? B-ENT 2016;12(1):17-21.

\section{Lee 2018 \{published data only\}}

Lee SM, Tenney R, Wallace A, Arjojmandi M. The end perioperative smoking pilot study: a randomized trial comparing e-cigarettes versus nicotine patch. Canadian Journal of Anesthesia 2017;64(1 Supplement 1):S48-S49.

* Lee SM, Tenney R, Wallace AW, Arjomandi M. E-cigarettes versus nicotine patches for perioperative smoking cessation: a pilot randomized trial. PeerJ 2018;6(9):e5609.

NCT02482233. A pilot randomized controlled clinical trial - "Electronic nicotine delivery device (e-cigarette) for perioperative smoking cessation in veterans". clinicaltrials.gov/ show/NCT02482233 (accessed 17 February 2016).

Lee 2019 \{published data only\}

KCT0001277. Effect of an electronic cigarette for smoking reduction and cessation in Korean male smokers: a randomized, controlled study. KCT0001277 2014 (accessed 15 August 2016).

* Lee SH, Ahn SH, Cheong YS. Effect of electronic cigarettes on smoking reduction and cessation in Korean male smokers: a randomized controlled study. Journal of the American Board of Family Medicine 2019;32(4):567-74.

\section{Lucchiari 2020 \{published data only\}}

Lucchiari C, Masiero M, Mazzocco K, Veronesi G, Maisonneauve $\mathrm{P}$, Jemos $\mathrm{C}$, et al. Benefits of e-cigarettes in smoking reduction and in pulmonary health among chronic smokers undergoing a lung cancer screening program at 6 months. Addictive Behaviours 2020;103:106222.

* Lucchiari C, Masiero M, Veronesi G, Maisonneuve P, Spina S, Jemos $C$, et al. Benefits of e-cigarettes among heavy smokers undergoing a lung cancer screening program: randomized controlled trial protocol. JMIR Research Protocols 2016;5(1):e21. [PMID: 26842790]

Masiero M, Lucchiari C, Mazzocco K, Veronesi G, Maisonneuve P, Jemos $\mathrm{C}$, et al. E-cigarettes may support smokers with high smoking-related risk awareness to stop smoking in the short run: preliminary results by randomized controlled trial. Nicotine and Tobacco Research 2019;21(1):119-126. [NCT02422914]

Masiero M, Lucchiari C, Mazzocco K, Veronesi G, Maisonneuve P Jemos $C$, et al. Corrigendum: E-cigarettes may support smokers with high smoking-related risk awareness to stop smoking in the short run: preliminary results by randomized controlled trial. Nicotine and Tobacco Research 2020;22(4):594-5. [NCT02422914]

NCT02422914. Benefits of tobacco free cigarette among heavy smokers undergoing a lung cancer screening program: a randomized controlled study. clinicaltrials.gov/show/ NCT02422914 (accessed 17 February 2016).

Martner 2019 \{published data only (unpublished sought but not used)\}

Martner SG, Dallery J. Technology-based contingency management and e-cigarettes during the initial weeks of a smoking quit attempt. Journal of Applied Behavior Analysis 2019;52(4):928-43.

McRobbie 2015 \{published data only\}

McRobbie H, Goniewicz M, Phillips A, Myers-Smith K, West O, Hajek P. Effects of the use of electronic cigarettes with and 
without concurrent smoking on acrolein delivery POS4-33. In: Society for Research on Nicotine and Tobacco, 20th Annual Meeting, Seattle, Washington. 2014:13. [www.srnt.org/ conferences/2014/pdf/SRNT_2014_Rapids_C.pdf]

* McRobbie H, Phillips A, Goniewicz ML, Smith KM, KnightWest O, Przulj D, et al. Effects of switching to electronic cigarettes with and without concurrent smoking on exposure to nicotine, carbon monoxide, and acrolein. Cancer Prevention Research (Philadelphia, Pa.) 2015;8(9):873-8.

NCT01714778. Toxins and Delivery in E-cigarette USers (TADEUS). clinicaltrials.gov/ct2/show/NCT01714778 (first received 26 October 2012).

\section{Meier 2017 \{published data only\}}

Meier E, Wahlquist AE, Heckman BW, Cummings KM, Froeliger B, Carpenter MJ. A pilot randomized crossover trial of electronic cigarette sampling among smokers. Nicotine \& Tobacco Research 2017;19(2):176-82.

\section{NCT02648178 \{published data only\}}

NCT02648178. Evaluation of appeal and impact of e-cigarettes among chronic smokers with smoking-related cancers. clinicaltrials.gov/show/NCT02648178 (accessed 17 February 2016).

\section{NCT02918630 \{published data only\}}

NCT02918630. E-cigarettes to promote smoking reduction among individuals with schizophrenia. clinicaltrials.gov/ct2/ show/NCT02918630 (first received 5 July 2018).

\section{Nides 2014 \{published data only\}}

NCT01898169. Evaluation of short-term safety and use patterns of an electronic nicotine delivery system. clinicaltrials.gov/ct2/ show/NCT01898169 (first received 12 July 2013).

* Nides MA, Leischow SJ, Bhatter M, Simmons M. Nicotine blood levels and short-term smoking reduction with an electronic nicotine delivery system. American Journal of Health Behavior 2014;38(2):265-74

\section{Oncken 2015 \{published data only\}}

NCT01775787. Effects of Electronic Cigarettes on Nicotine Concentrations (ECIG). clinicaltrials.gov/ct2/show/ NCT01775787 (first received 25 January 2013).

* Oncken CA, Litt MD, McLaughlin LD, Burki NA. Nicotine concentrations with electronic cigarette use: effects of sex and flavor. Nicotine \& Tobacco Research 2015;17(4):473-8.

Swedeh MA, Oncken C, Burki NK. Acute effects of electronic cigarettes on airway function in human subjects. In: American Thoracic Society International Conference Abstracts. Vol. 189. 2014:A4089.

\section{Ozga-Hess 2019 \{published data only\}}

Ozga-Hess JE, Felicione NJ, Ferguson SG, Dino G, Elswick D, Whitworth $\mathrm{C}$, et al. Piloting a clinical laboratory method to evaluate the influence of potential modified risk tobacco products on smokers' quit-related motivation, choice, and behaviour. Addictive Behaviors 2019;99:106105.

\section{Pacifici 2015 \{published data only\}}

Pacifici R, Pichini S, Graziano S, Pellegrini M, Massaro G, Beatrice F. Successful nicotine intake in medical assisted use of e-cigarettes: a pilot study. International Journal of Environmental Research and Public Health 2015;12(7):7638-46.

Polosa 2011 \{published data only\} NCT01195597. Smoking cessation and reduction with an electronic nicotine delivery device (ENDD). clinicaltrials.gov/ ct2/show/NCT01195597 (first received 6 September 2010).

* Polosa R, Caponnetto P, Morjaria JB, Papale G, Campagna D, Russo C. Effect of an electronic nicotine delivery device (ecigarette) on smoking reduction and cessation: a prospective 6month pilot study. BMC Public Health 2011;11:786.

Polosa R, Morjaria JB, Caponnetto P, Campagna D, Russo C Alamo A, et al. Effectiveness and tolerability of electronic cigarette in real-life: a 24-month prospective observational study. Internal and Emergency Medicine 2014;9(5):537-46.

Polosa 2014b \{published data only\} NCT02124200. High Cessation Rates in Smokers Using Personal Vaporizers (VAPECIG). clinicaltrials.gov/ct2/show/NCT02124200 (first received 28 April 2014).

Polosa R, Caponnetto P, Maglia M, Morjaria JB, Russo C. Success rates with nicotine personal vaporizers: a prospective 6-month pilot study of smokers not intending to quit. BMC Public Health 2014;14:1159.

Polosa 2015 \{published data only\}

Polosa R, Caponnetto P, Cibella F, Le-Houezec J. Quit and smoking reduction rates in vape shop consumers: a prospective 12-month survey. International Journal of Environmental Research and Public Health 2015;12(4):3428-38.

\section{Pratt 2016 \{published data only\}}

Pratt SI, Sargent J, Daniels L, Santos MM, Brunette M. Appeal of electronic cigarettes in smokers with serious mental illness. Addictive Behaviors 2016;59:30-4.

Pulvers 2018 \{published data only\}

Pulvers K, Emami AS, Nollen NL, Romero DR, Strong DR, Benowitz NL, et al. Tobacco consumption and toxicant exposure of cigarette smokers using electronic cigarettes. Nicotine \& Tobacco Research 2018;20(2):206-14.

Pulvers 2020 \{published data only\}

Pulvers K, Nollen NL, Rice M, Schmid CH, Qu K, Benowitz NL, et al. Effect of pod e-cigarettes vs cigarettes on carcinogen exposure among African American and Latinx smokers: a randomized clinical trial. JAMA NetwOrk Open 2020;3(11):e2026324.

\section{Scheibein 2020 \{published data only\}}

Scheibein F, McGirr K, Morrison A, Roche, Wells JS. An exploratory non-randomized study of a 3-month electronic nicotine delivery system (ENDS) intervention with people accessing a homeless supported temporary accommodation service (STA) in Ireland. Harm Reduction Journal 2020;17(1):73. 
Smith 2020 \{published and unpublished data\}

Smith TT, Heckman BW, Wahlquist AE, Cummings KM, Carpenter MJ. The impact of e-liquid propylene glycol and vegetable glycerin ratio on ratings of subjective effects, reinforcement value, and use in current smokers. Nicotine \& Tobacco Research 2020;22(5):791-7.

\section{Stein 2016 \{published data only\}}

Stein MD, Caviness C, Grimone K, Audet D, Anderson BJ, Bailey GL. An open trial of electronic cigarettes for smoking cessation among methadone-maintained smokers. Nicotine \& Tobacco Research 2016;18(5):1157-62.

\section{Strasser 2016 \{published data only\}}

Strasser AA, Souprountchouk V, Kaufmann A, Blazekovic S, Leone F, Benowitz NL, et al. Nicotine replacement, topography, and smoking phenotypes of e-cigarettes. Tobacco Regulatory Science 2016;2(4):352-62.

\section{Tseng 2016 \{published data only\}}

NCT02628964. Assessing the use of electronic cigarettes (ecigarettes) as a harm reduction strategy. clinicaltrials.gov/show/ NCT02628964 (accessed 17 February 2016).

* Tseng TY, Ostroff JS, Campo A, Gerard M, Kirchner T, Rotrosen $\mathrm{J}$, et al. A randomized trial comparing the effect of nicotine versus placebo electronic cigarettes on smoking reduction among young adult smokers. Nicotine \& Tobacco Research 2016;18(10):1937-43.

\section{Valentine 2018 \{published data only\}}

Valentine GW, Hefner K, Jatlow PI, Rosenheck RA, Gueorguieva R, Sofuoglu M. Impact of e-cigarettes on smoking and related outcomes in veteran smokers with psychiatric comorbidity. Journal of Dual Diagnosis 2018;14(1):2-13.

\section{Van Staden 2013 \{published data only\}}

Van Staden SR, Groenewald M, Engelbrecht R, Becker PJ, Hazelhurst LT. Carboxyhaemoglobin levels, health and lifestyle perceptions in smokers converting from tobacco cigarettes to electronic cigarettes. South African Medical Journal 2013;103(11):865-8.

\section{Veldheer 2019 \{published and unpublished data\}}

Lopez AA, Cobb CO, Yingst JM, Veldheer S, Hrabovsky S, Yen MS, et al. A transdisciplinary model to inform randomized clinical trial methods for electronic cigarette evaluation. BMC Public Health 2016;16(1):217. [PMCID:: PMC4778292] [PMID: 26941050]

NCT02342795. Randomized controlled trial methods for novel tobacco products evaluation. clinicaltrials.gov/show/ NCT02342795 (accessed 17 February 2016).

* Veldheer S, Yingst J, Midya V, Hummer B, Lester C, Krebs N, . Pulmonary and other health effects of electronic cigarette use among adult smokers participating in a randomized controlled smoking reduction trial. Addictive Behaviors 2019;91:95-101.

Yingst J, Foulds J, Veldheer S, Cobb CO, Yen M, Hrabovsky S, et al. Measurement of electronic cigarette frequency of use among smokers participating in a randomized controlled trial. Nicotine \& Tobacco Research 2020;22(5):699-704. [NCT02342795]

\section{Wadia 2016 \{published data only\}}

Wadia R, Booth V, Yap HF, Moyes DL. A pilot study of the gingival response when smokers switch from smoking to vaping. British Dental Journal 2016;221(11):722-6.

\section{Walele 2018 \{published data only\}}

Cravo AS, Bush J, Sharma G, Savioz R, Martin C, Craige S, et al. A randomised, parallel group study to evaluate the safety profile of an electronic vapour product over 12 weeks. Regulatory Toxicology and Pharmacology 2016;81:S1-S14.

NCT02029196. A randomised, parallel group, multi-centre study to evaluate the safety profile of the ITG EVP G1 product. clinicaltrials.gov/show/NCT02029196 (accessed 16 July 2014).

NCT02143310. A multi-centre study to evaluate the safety of use of electronic vapour products for two years. clinicaltrials.gov/ show/NCT02143310 (accessed 16 July 2014).

* Walele T, Bush J, Koch A, Savioz R, Martin C, O'Connell G. Evaluation of the safety profile of an electronic vapour product used for two years by smokers in a real-life setting. Regulatory Toxicology and Pharmacology 2018;92:226-38.

Walker 2020 \{published data only\}

NCT02521662. A randomised-controlled clinical trial to evaluate the effectiveness and safety of combining nicotine patches with e-cigarettes (with and without nicotine) plus behavioural support, on smoking abstinence. clinicaltrials.gov/show/ NCT02521662 (accessed 17 February 2016).

* Walker N, Parag V, Verbiest M, Laking G, Laugesen M, Bullen C. Nicotine patches used in combination with e-cigarettes (with and without nicotine) for smoking cessation: a pragmatic, randomised trial. Lancet. Respiratory Medicine 2020;8(1):54-64.

Walker N, Verbiest M, Kurdziel T, Laking G, Laugesen M, Parag V, et al. Effectiveness and safety of nicotine patches combined with e-cigarettes (with and without nicotine) for smoking cessation: study protocol for a randomised controlled trial. BMJ Open 2019;9(2):e023659.

\section{Yingst 2020 \{published data only\}}

Yingst J, Foulds J, Zurlo J, Steinberg MB, Eissenberg T, Du P. Acceptability of electronic nicotine delivery systems (ENDS) among HIV positive smokers. AIDS Care 2020;32(10):1224-8.

\section{References to studies excluded from this review}

\section{Adkison 2013 \{published data only\}}

Adkison SE, O'Connor RJ, Bansal-Travers M, Hyland A, Borland $\mathrm{R}$, Yong $\mathrm{HH}$, et al. Electronic nicotine delivery systems: international tobacco control four-country survey. American Journal of Preventive Medicine 2013;44(3):207-15.

\section{Al-Delaimy 2015 \{published data only\}}

* Al-Delaimy WK, Myers MG, Leas EC, Strong DR, Hofstetter CR. $\mathrm{E}$-cigarette use in the past and quitting behavior in the future: a population-based study. American Journal of Public Health 2015;105(6):1213-9. 
Donzelli A. E-cigarettes may impair ability to quit, but other explanations are possible. American Journal of Public Health 2015;105(11):e1.

\section{Anonymous 2019 \{published data only\}}

Anonymous. E-cigarettes best other cessation tools. Cancer Discovery 2019;9(4):OF3.

\section{Battista 2013 \{published data only\}}

Battista L, Di lorio M, Tancredi M, Acconcia MC, Torromeo C, Barilla F, et al. Cardiovascular effects of electronic cigarettes. Circulation 2013;128:A16755.

\section{Bianco 2019 \{published data only\}}

Bianco CL, Pratt SI, Ferron JC, Brunette MF. Electronic cigarette use during a randomized trial of interventions for smoking cessation among Medicaid beneficiaries with mental illness. Journal of Dual Diagnosis 2019;15(3):184-91.

\section{Biener 2015 \{published data only\}}

Biener L, Hargraves JL. A longitudinal study of electronic cigarette use among a population-based sample of adult smokers: association with smoking cessation and motivation to quit. Nicotine \& Tobacco Research 2015;17(2):127-33.

\section{Biondi-Zoccai 2019 \{published data only\}}

Biondi-Zoccai G, Sciarretta S, Bullen C, Nocella C, Violi F, Loffredo L, et al. Acute effects of heat-not-burn, electronic vaping, and traditional tobacco combustion cigarettes: the Sapienza University of Rome-Vascular Assessment of Proatherosclerotic Effects of Smoking ( SUR - VAPES ) 2 randomized trial. Journal of the American Heart Association 2019;8(6):e010455.

\section{Biondi-Zoccai 2020 \{published data only\}}

Biondi-Zoccai G, Carnevale R, Vitali M, Tritapepe L, Martinelli O, Macrina $F$, et al. A randomized trial comparing the acute coronary, systemic, and environmental effects of electronic vaping cigarettes versus heat-not-burn cigarettes in smokers of combustible cigarettes undergoing invasive coronary assessment: rationale and design of the SUR-VAPES 3 trial. Minerva Cardioangiologica 2020;68(6):548-55.

\section{Borderud 2014 \{published data only\}}

Barton MK. Electronic cigarettes did not help patients with cancer stop smoking. CA: a cancer journal for clinicians 2015;65(2):85-6.

* Borderud SP, Li Y, Burkhalter JE, Sheffer CE, Ostroff JS. Electronic cigarette use among patients with cancer: characteristics of electronic cigarette users and their smoking cessation outcomes. Cancer 2014;120(22):3527-35.

Fillon M. Electronic cigarettes might not help cancer patients quit smoking. Journal of the National Cancer Institute 2015;107(1):496

\section{Brose 2015 \{published data only\}}

* Brose LS, Hitchman SC, Brown J, West R, McNeill A. Is the use of electronic cigarettes while smoking associated with smoking cessation attempts, cessation and reduced cigarette consumption? A survey with a 1-year follow-up. Addiction 2015;110(7):1160-8.

Brown J, West R, Beard E, Michie S, Shahab L, McNeill A. Prevalence and characteristics of e-cigarette users in Great Britain: Findings from a general population survey of smokers. Addictive Behaviors 2014;39(6):1120-5.

Hitchman SC, Brose LS, Brown J, Robson D, McNeill A. Associations between e-cigarette type, frequency of use, and quitting smoking: findings from a longitudinal online panel survey in Great Britain. Nicotine \& Tobacco Research 2015;17(10):1187-94.

Brown 2014a \{published data only\}

Brown J, Beard E, Kotz D, Michie S, West R. Real-world effectiveness of e-cigarettes when used to aid smoking cessation: a cross-sectional population study. Addiction 2014;109(9):1531-40.

\section{Bullen 2010 \{published data only\}}

Bullen C, McRobbie H, Thornley S, Glover M, Lin R, Laugesen M. Effect of an electronic nicotine delivery device (e cigarette) on desire to smoke and withdrawal, user preferences and nicotine delivery: randomised cross-over trial. Tobacco Control 2010;19(2):98-103.

\section{Bullen 2018 \{published data only\}}

Bullen C, Verbiest M, Galea-Singer S, Kurdziel T, Laking G, Newcombe D, et al. The effectiveness and safety of combining varenicline with nicotine e-cigarettes for smoking cessation in people with mental illnesses and addictions: study protocol for a randomised-controlled trial. BMC Public Health 2018;18(1):596.

\section{Caponnetto 2019 \{published data only\}}

Caponnetto P, Maglia M, Polosa R. Efficacy of smoking cessation with varenicline plus counselling for e-cigarettes users (VAREVAPE): a protocol for a randomized controlled trial. Contemporary Clinical Trials Communications 2019;15:100412.

Cavarretta 2019 \{published data only\}

Cavarretta E, Sciarretta S, Nocella C, Peruzzi M, Marullo AG, Loffredo $L$, et al. Subjective smoking satisfaction between heat-not-burn, electronic vaping, and traditional tobacco combustion cigarettes: a sub-analysis of the SUR-VAPES 2 trial. European Journal of Preventive Cardiology 2019;26(Supplement 1):S114.

\section{Chaumont 2018 \{published data only\}}

Chaumont M, de Becker B, Zaher W, Culie A, Deprez G, Melot C, et al. Differential Effects of E-Cigarette on Microvascular Endothelial Function, Arterial Stiffness and Oxidative Stress: a Randomized Crossover Trial. Scientific reports 2018;8(1):10378.

\section{Chaumont 2019 \{published data only\}}

Chaumont M, El Channan M, Bernard A, Lesage A, Deprez G Van Muylem A, et al. Short-term high wattage e-cigarette cessation improves cardiorespiratory outcomes in regular users: a randomized crossover trial. Journal of Hypertension 2019; Conference: 29th European Meeting on Hypertension 
and Cardiovascular Protection, ESH 2019. Italy.

37(Supplement 1):e8-9.

\section{Chausse 2015 \{published data only\}}

Chausse P, Naughton G, Dutheil F. Electronic cigarettes: the resistance value of the heating filament could be the key to lung toxicity. Chest 2015;148(1):e29-30.

Choi 2014 \{published data only\}

Choi K, Forster JL. Beliefs and experimentation with electronic cigarettes: a prospective analysis among young adults. American Journal of Preventive Medicine 2014;6(2):175-8.

* Choi K Forster JL. Authors' Response to: Context on use is needed before public health recommendations are made about e-cigarettes. Americal Journal of Preventive Medicine 2014;46(6):e58-59.

\section{Chorti 2012 \{published data only\}}

Chorti M, Poulianiti K, Jamurtas A, Kostikas K, Tzatzarakis M, Vynias D, et al. Effects of active and passive electronic and tobacco cigarette smoking on lung function (P08-02). Toxicology Letters 2012;211:S64.

\section{Collins 2019 \{published data only\}}

Collins SE, Nelson LA, Stanton J, Mayberry N, Ubay T, Taylor EM, et al. Harm reduction treatment for smoking (HaRT-S): findings from a single-arm pilot study with smokers experiencing chronic homelessness. Substance Abuse 2019;40(2):229-39.

\section{Cook 2019 \{published data only\}}

Cook R, Davidson P, Martin R. E-cigarettes helped more smokers quit than nicotine replacement therapy. BMJ 2019;365:l2036.

\section{Cox 2019a \{published data only\}}

Cox S, Dawkins L, Doshi J, Cameron J. Effects of e-cigarettes versus nicotine replacement therapy on short-term smoking abstinence when delivered at a community pharmacy. Addictive Behaviors Reports 2019;10:100202.

\section{Czogala 2012 \{published data only\}}

Czogala J, Cholewinski M, Kutek A, Zielinska-Danch W. Evaluation of changes in hemodynamic parameters after the use of electronic nicotine delivery systems among regular cigarette smokers. Przeglad Lekarski 2012;69(10):841-5.

\section{D'Ruiz 2017 \{published data only\}}

D'Ruiz CD, O'Connell G, Graff DW, Yan XS. Measurement of cardiovascular and pulmonary function endpoints and other physiological effects following partial or complete substitution of cigarettes with electronic cigarettes in adult smokers. Regulatory Toxicology and Pharmacology 2017;87:36-53.

\section{Dawkins 2012 \{published data only\}}

Dawkins L, Turner J, Hasna S, Soar K. The electronic-cigarette: effects on desire to smoke, withdrawal symptoms and cognition. Addictive Behaviors 2012;37(8):970-3.

\section{Dawkins 2013a \{published data only\}}

Dawkins L, Turner J, Crowe E. Nicotine derived from the electronic cigarette improves time-based prospective memory in abstinent smokers. Psychopharmacology 2013;227(3):377-84.

Dawkins 2014 \{published data only\}

Dawkins L, Corcoran O. Acute electronic cigarette use: nicotine delivery and subjective effects in regular users. Psychopharmacology 2014;231(2):401-7.

Douptcheva 2013 \{published data only\}

Douptcheva N, Gmel G, Studer J, Deline S, Etter JF. Use of electronic cigarettes among young Swiss men. Journal of Epidemiology \& Community Health 2013;67(12):1075-6.

Dutra 2014 \{published data only\}

Dutra LM, Glantz SA. Electronic cigarettes and conventional cigarette use among U.S. adolescents: a cross-sectional study. JAMA Pediatrics 2014;168(7):610-7.

\section{Eissenberg 2010 \{published data only\}}

Eissenberg T. Electronic nicotine delivery devices: ineffective nicotine delivery and craving suppression after acute administration. Tobacco Control 2010;19(1):87-8.

\section{Etter 2014 \{published data only\}}

Etter JF, Bullen C. A longitudinal study of electronic cigarette users. Addictive Behaviors 2014;39(2):491-4.

\section{Farsalinos 2012 \{published data only\}}

* Farsalinos K, Tsiapras D, Kyrzopoulos S, Savvopoulou M, Avramidou E, Vasilopoulou D, et al. Acute effects of using an electronic nicotine-delivery device (e-cigarette) on myocardial function: comparison with the effects of regular cigarettes. European Heart Journal 2012;33:203.

Farsalinos KE, Romagna G, Tsiapras D, Kyrzopoulos S, Voudris V. Acute effects of using an electronic nicotine-delivery device (electronic cigarette) on myocardial function: comparison with the effects of regular cigarettes. BMC Cardiovascular Disorders 2014;14:78

\section{Farsalinos 2013a \{published data only\}}

Farsalinos KE, Romagna G, Tsiapras D, Kyrzopoulos S, Voudris V. Evaluating nicotine levels selection and patterns of electronic cigarette use in a group of "vapers" who had achieved complete substitution of smoking. Substance Abuse: Research and Treatment 2013;7:139-46.

\section{Farsalinos 2013b \{published data only\}}

Farsalinos KE, Romagna G, Tsiapras D, Kyrzopoulos S, Spyrou A, Voudris V. Impact of flavour variability on electronic cigarette use experience: an internet survey. International Journal of Environmental Research \& Public Health 2013;10(12):7272-82.

\section{Farsalinos 2013c \{published data only\}}

Farsalinos K, Tsiapras D, Kyrzopoulos S, Stefopoulos C, Spyrou A, Tsakalou M, et al. Immediate effects of electronic cigarette use on coronary circulation and blood carboxyhemoglobin levels: comparison with cigarette smoking. European Heart Journal 2013;34(Suppl 1):13. 


\section{Farsalinos 2013d \{published data only\}}

Farsalinos K, Tsiapras D, Kyrzopoulos S, Spyrou A, Stefopoulos C, Romagna G, et al. Effects of electronic cigarette use on the elastic properties of the ascending aorta in healthy subjects: comparison with the effects of tobacco cigarettes. European Heart Journal Cardiovascular Imaging 2013;14(Suppl 2):ii203.

\section{Flouris 2012 \{published data only\}}

Flouris AD, Poulianiti KP, Chorti MS, Jamurtas AZ, Kouretas D, Owolabi EO, et al. Acute effects of electronic and tobacco cigarette smoking on complete blood count. Food and Chemical Toxicology 2012;50(10):3600-3.

\section{Flouris 2013 \{published data only\}}

Flouris AD, Chorti MS, Poulianiti KP, Jamurtas AZ, Kostikas K, Tzatzarakis MN, et al. Acute impact of active and passive electronic cigarette smoking on serum cotinine and lung function. Inhalation Toxicology 2013;25(2):91-101.

\section{Gmel 2016 \{published data only\}}

Gmel G, Baggio S, Mohler-Kuo M, Daeppen JB, Studer J. E-cigarette use in young Swiss men: is vaping an effective way of reducing or quitting smoking? Swiss Medical Weekly 2016;146:w14271.

\section{Gottlieb 2019 \{published data only\}}

Gottlieb MA. E-cigarettes versus nicotine-replacement therapy for smoking cessation. New England Journal of Medicine 2019;380(20):1974.

\section{Grana 2014b \{published data only\}}

Grana RA, Popova L, Ling PM. A longitudinal analysis of electronic cigarette use and smoking cessation. JAMA Internal Medicine 2014;174(5):812-3.

\section{James 2016 \{published data only\}}

* James SA, Meier EM, Wagener TL, Smith KM, Neas BR, Beebe LA. E-Cigarettes for immediate smoking substitution in women diagnosed with cervical dysplasia and associated disorders. International Journal of Environmental Health Research 2016;13(3):E288. [DOI: 10.3390/ijerph13030288]

NCT01842828. E-Cigarettes as an addition to multicomponent treatment for tobacco dependence: a pilot study. clinicaltrials.gov/show/NCT01842828 (accessed 16 July 2014).

\section{Kasza 2013 \{published data only\}}

Kasza KA, Bansal-Travers M, O'Connor RJ, Compton WM, Kettermann A, Borek N, et al. Cigarette smokers' use of unconventional tobacco products and associations with quitting activity: findings from the ITC-4 U.S. cohort. Nicotine \& Tobacco Research 2013;16(6):672-81.

\section{Kouretas 2012 \{published data only\}}

Kouretas D, Poulianiti K, Chorti M, Jamurtas A, Kostikas K, Tzatzarakis $\mathrm{M}$, et al. Effects of electronic cigarette and tobacco cigarette smoking on complete blood count (P08-03). Toxicology Letters 2012;211:S64.
Kousta 2019 \{published data only\}

Kousta S. E-cigarettes for smoking cessation. Nature Human Behaviour 2019;3:322.

\section{Lechner 2015 \{published data only\}}

Lechner WV, Meier E, Wiener JL, Grant DM, Gilmore J, Judah MR, et al. The comparative efficacy of first- versus secondgeneration electronic cigarettes in reducing symptoms of nicotine withdrawal. Addiction 2015;110(5):862-7.

\section{Lee 2014 \{published data only\}}

Lee S, Grana RA, Glantz SA. Electronic cigarette use among Korean adolescents: a cross-sectional study of market penetration, dual use, and relationship to quit attempts and former smoking. Journal of Adolescent Health 2014;54(6):684-90.

\section{Manzoli 2015 \{published data only\}}

* Manzoli L, Flacco ME, Fiore M, La Vecchia C, Marzuillo C, Gualano MR, et al. Electronic cigarettes efficacy and safety at 12 months: cohort study. PLOS One 2015;10(6):e0129443.

Manzoli L, La Vecchia C, Flacco ME, Capasso L, Simonetti V, Boccia S, et al. Multicentric cohort study on the long-term efficacy and safety of electronic cigarettes: study design and methodology. BMC Public Health 2013;13(1):883.

NCT01785537. The efficacy and safety of electronic cigarettes: a 5-year follow-up study. clinicaltrials.gov/ct2/show/ NCT01785537 (first received 7 February 2013).

\section{Marini 2014 \{published data only\}}

Marini S, Buonanno G, Stabile L, Ficco G. Short-term effects of electronic and tobacco cigarettes on exhaled nitric oxide. Toxicology and Applied Pharmacology 2014;278(1):9-15.

\section{Mayor 2019 \{published data only\}}

Mayor S. E-cigarettes help twice as many smokers quit as nicotine replacement therapy, trial finds. BMJ 2019;364:1473.

\section{Meltzer 2017 \{published data only\}}

Meltzer LR, Simmons VN, Sutton SK, Drobes DJ, Quinn GP, Meade $C D$, et al. A randomized controlled trial of a smoking cessation self-help intervention for dual users of tobacco cigarettes and e-cigarettes: intervention development and research design. Contemporary Clinical Trials 2017;60:56-62.

\section{Miura 2015 \{published data only\}}

Miura N, Yuki D, Minami N, Kakehi A, Futama Y. A study to investigate changes in the levels of biomarkers of exposure to selected cigarette smoke constituents in Japanese adult male smokers who switched to a non-combustion inhaler type of tobacco product. Regulatory Toxicology and Pharmacology 2015;71(3):498-506.

\section{NCT02487953 \{published data only\}}

NCT02487953. Electronic Nicotine Delivery Systems (ENDS) as a smoking cessation treatment. clinicaltrials.gov/ct2/show/ NCT02487953 (first received 2 July 2015). 


\section{NCT03036644 \{published data only\}}

NCT03036644. Comparison of electronic cigarettes and tobacco cigarettes on cardiovascular function and oxidative stress. clinicaltrials.gov/ct2/show/NCT03036644 (first received 30 January 2017).

\section{NCT03575468 \{published data only\}}

NCT03575468. Enhanced e-cigarette coaching intervention for dual users of cigarettes and e-cigarettes. clinicaltrials.gov/ct2/ show/NCT03575468 (first received 2 July 2018).

\section{NCT04107779 \{published data only\}}

NCT04107779. Changes in biomarkers of cigarette smoke exposure after switching either exclusively or partly to JUUL ENDS. clinicaltrials.gov/ct2/show/NCT04107779 (first received 27 September 2019).

\section{Nolan 2016 \{published data only\}}

Nolan M, Leischow S, Croghan I, Kadimpati S, Hanson A, Schroeder D, et al. Feasibility of electronic nicotine delivery systems in surgical patients. Nicotine \& Tobacco Research 2016;18(8):1757-62.

\section{NTR6224 \{published data only\}}

NTR6224. Electronic cigarettes: an intervention for dual-users. www.who.int/trialsearch/Trial2.aspx?TrialID=NTR6224 (first received 12 January 2017).

\section{Palamidas 2014 \{published data only\}}

Palamidas A, Gennimata SA, Kaltsakas G, Tsikrika S, Vakali S, Gratziou C, et al. Acute effect of an e-cigarette with and without nicotine on lung function. Tobacco Induced Diseases 2014;12(Suppl 1):A34.

\section{Pearson 2012 \{published data only\}}

Pearson JL, Richardson A, Niaura RS, Vallone DM, Abrams DB. E-cigarette awareness, use, and harm perceptions in US adults. American Journal of Public Health 2012;102(9):1758-66.

\section{Pokhrel 2013 \{published data only\}}

Pokhrel P, Fagan P, Little MA, Kawamoto CT, Herzog TA. Smokers who try e-cigarettes to quit smoking: findings from a multiethnic study in Hawaii. American Journal of Public Health 2013;103(9):e57-62.

\section{Polosa 2014a \{published data only\}}

Polosa R, Morjaria J, Caponnetto P, Caruso M, Strano S, Battaglia $\mathrm{E}$, et al. Effect of smoking abstinence and reduction in asthmatic smokers switching to electronic cigarettes: evidence for harm reversal. International Journal of Environmental Research \& Public Health 2014;11(5):4965-77.

\section{Popova 2013 \{published data only\}}

Ling PM, Popova L. Novel "Tobacco" product use and association with smoking cessation: a national study. Journal of General Internal Medicine 2012;27(2 Suppl):S254.

\footnotetext{
* Popova L, Ling PM. Alternative tobacco product use and smoking cessation: a national study. American Journal of Public Health 2013;103(5):923-30.
}

\section{Prochaska 2014 \{published data only\}}

Prochaska JJ, Grana RA. E-cigarette use among smokers with serious mental illness. PLOS One 2014;9(11):e113013.

Russo 2018 \{published data only\}

Russo C, Cibella F, Mondati E, Caponnetto P, Frazzetto E, Caruso M, et al. Lack of substantial post-cessation weight increase in electronic cigarettes users. International Journal of Environmental Research and Public Health 2018;15(4):581.

Schober 2014 \{published data only\}

Schober W, Szendrei K, Matzen W, Osiander-Fuchs H, Heitmann D, Schettgen T, et al. Use of electronic cigarettes (ecigarettes) impairs indoor air quality and increases FeNO levels of e-cigarette consumers. International Journal of Hygiene and Environmental Health 2014;217(6):628-37.

\section{Siegel 2011 \{published data only\}}

Siegel MB, Tanwar KL, Wood KS. Electronic cigarettes as a smoking-cessation tool: results from an online survey. American Journal of Preventive Medicine 2011;40(4):472-5.

\section{Song 2020 \{published data only\}}

Song M-A, Reisinger SA, Freudenheim JL, Brasky TM, Mathe EA, McElroy JP, et al. Effects of electronic cigarette constituents on the human lung: a pilot clinical trial. Cancer Prevention Research 2020;13(2):145-52.

\section{St. Helen 2020 \{published data only\}}

StHelen G, Nardone N, Addo N, Dempsey D, Havel C, Jacob P, et al. Differences in nicotine intake and effects from electronic and combustible cigarettes among dual users. Addiction 2020;115(4):757-67.

\section{Stein 2019 \{published data only\}}

Stein JH, Korcarz CE. E-cigarettes versus nicotine-replacement therapy for smoking cessation. New England Journal of Medicine 2019;380(20):1973-4.

\section{Stower 2019 \{published data only\}}

Stower H. E-cigarettes to help smoking cessation. Nature Medicine 2019;25(3):358.

\section{Tsikrika 2014 \{published data only\}}

Tsikrika S, Vakali S, Gennimata SA, Palamidas A, Kaltsakas G, Koulouris N, et al. Short term use of an e-cig: influence on clinical symptoms, vital signs and eCO levels. Tobacco Induced Diseases 2014;12(Suppl 1):A30.

\section{Tucker 2018 \{published data only\}}

Tucker MR, Laugesen M, Bullen C, Grace RC. Predicting shortterm uptake of electronic cigarettes: effects of nicotine, subjective effects, and simulated demand. Nicotine \& Tobacco Research 2018;20(10):1265-71.

\section{Tzatzarakis 2013 \{published data only\}}

Tzatzarakis MN, Tsitoglou KI, Chorti MS, Poulianiti KP, Jamurtas AZ, Koutedakis $\mathrm{Y}$, et al. Acute and short term impact of active and passive tobacco and electronic cigarette smoking on inflammatory markers. Toxicology Letters 2013;221(Suppl):S86. 
Vakali 2014 \{published data only\}

Vakali S, Tsikrika S, Gennimata SA, Kaltsakas G, Palamidas A, Koulouris N, et al. E-cigarette acute effect on symptoms and airway inflammation: comparison of nicotine with a nonnicotine cigarette. Tobacco Induced Diseases 2014;12(Suppl 1):A35.

\section{Valentine 2016 \{published data only\}}

Valentine GW, Jatlow PI, Coffman M, Nadim H, Gueorguieva R, Sofuoglu M. The effects of alcohol-containing e-cigarettes on young adult smokers. Drug and Alcohol Dependence 2016;159:272-6.

\section{Van Heel 2017 \{published data only\}}

Van Heel M, Van Gucht D, Vanbrabant K, Baeyens F. The importance of conditioned stimuli in cigarette and e-cigarette craving reduction by e-cigarettes. International Journal of Environmental Research and Public Health 2017;14(2):193.

\section{Vansickel 2010 \{published data only\}}

Vansickel AR, Cobb CO, Weaver MF, Eissenberg TE. A clinical laboratory model for evaluating the acute effects of electronic "cigarettes": nicotine delivery profile and cardiovascular and subjective effects. Cancer Epidemiology, Biomarkers \& Prevention 2010;19(8):1945-53.

\section{Vansickel 2012 \{published data only\}}

Vansickel AR, Weaver MF, Eissenberg T. Clinical laboratory assessment of the abuse liability of an electronic cigarette. Addiction 2012;107(8):1493-500.

\section{Vansickel 2013 \{published data only\}}

Vansickel AR, Eissenberg T. Electronic cigarettes: effective nicotine delivery after acute administration. Nicotine \& Tobacco Research 2013;15(1):267-70.

\section{Vardavas 2012 \{published data only\}}

Vardavas $\mathrm{Cl}$, Anagnostopoulos N, Kougias M, Evangelopoulou V, Connolly GN, Behrakis PK. Short-term pulmonary effects of using an electronic cigarette: Impact on respiratory flow resistance, impedance, and exhaled nitric oxide. Chest 2012;141(6):1400-6.

\section{Vickerman 2013 \{published data only\}}

Vickerman KA, Carpenter KM, Altman T, Nash CM, Zbikowski SM. Use of electronic cigarettes among state tobacco cessation quitline callers. Nicotine \& Tobacco Research 2013;15(10):1787-91.

\section{Voos 2019 \{published data only\}}

Voos N, Kaiser L, Mahoney MC, Bradizza CM, Kozlowski LT, Benowitz NL, et al. Randomized within-subject trial to evaluate smokers' initial perceptions, subjective effects and nicotine delivery across six vaporized nicotine products. Addiction 2019;114(7):1236-48.

\section{Voos 2020 \{published data only\}}

Voos N, Smith D, Kaiser L, Mahoney MC, Bradizza CM, Kozlowski LT, et al. Effect of e-cigarette flavors on nicotine delivery and puffing topography: results from a randomized clinical trial of daily smokers. Psychopharmacology 2020;237(2):491-502.

Wagener 2014 \{published data only\}

Wagener TL, Meier E, Hale JJ, Oliver ER, Warner ML, Driskill LM, et al. Pilot investigation of changes in readiness and confidence to quit smoking after e-cigarette experimentation and 1 week of use. Nicotine \& Tobacco Research 2014;16(1):108-14.

Walele 2016a \{published data only\}

Walele T, Sharma G, Savioz R, Martin C, Williams J. A randomised, crossover study on an electronic vapour product, a nicotine inhalator and a conventional cigarette. Part A: Pharmacokinetics. Regulatory Toxicity 2016;74:187-92.

Walele 2016b \{published data only\}

Walele T, Sharma G, Savioz R, Martin C, Williams J. A randomised, crossover study on an electronic vapour product, a nicotine inhalator and a conventional cigarette. Part B: Safety and subjective effects. Regulatory Toxicity 2016;74:193-9.

Yan 2015 \{published data only\}

Yan XS, D'Ruiz C. Effects of using electronic cigarettes on nicotine delivery and cardiovascular function in comparison with regular cigarettes. Regulatory Toxicology and Pharmacology 2015;71(1):24-34.

Yuki 2017 \{published data only\}

Yuki D, Sakaguchi C, Kikuchi A, Futamura Y. Pharmacokinetics of nicotine following the controlled use of a prototype novel tobacco vapor product. Regulatory Toxicology and Pharmacology 2017;87(2):30-5.

\section{Zhang 2019 \{published data only\}}

Zhang Y, Upson D. E-cigarettes versus nicotine-replacement therapy for smoking cessation. New England Journal of Medicine 2019;380(20):1973.

\section{References to ongoing studies}

\section{ACTRN12617001324303 \{published data only\}}

ACTRN12617001324303. Vaporised nicotine products versus oral forms of nicotine replacement therapy (NRT) products for tobacco smoking cessation among low-socioeconomic status (low-SES) smokers. anzctr.org.au/ACTRN12617001324303.aspx (first received 15 September 2017).

\section{ACTRN12618000408280 \{published data only\}}

ACTRN12618000408280. Cessation and Relapse Prevention (CARP) Trial: Nicotine vaporisers compared to standard nicotine replacement therapy for smoking cessation among people with co-morbidities. www.anzctr.org.au/ ACTRN12618000408280.aspx (first received 21 March 2018).

\section{ACTRN12619001787178 \{published data only\}}

ACTRN12619001787178. Project NEAT: nicotinE As Treatment for tobacco smoking following discharge from residential withdrawal services. www.who.int/trialsearch/Trial2.aspx? TrialID=ACTRN12619001787178 (first received 18 November 2019). 
Begh 2019 \{published data only\}

Begh R, Coleman T, Yardley L, Barnes R, Naughton F, Gilbert H, et al. Examining the effectiveness of general practitioner and nurse promotion of electronic cigarettes versus standard care for smoking reduction and abstinence in hardcore smokers with smoking-related chronic disease: protocol for a randomised controlled trial. Trials 2019;20(1):659.

ISRCTN59404712. GP/nurse promotion of e-cigarettes in supporting reduced smoking and cessation in smokers. www.isrctn.com/ISRCTN59404712 (first received 28 November 2017).

\section{Berlin 2019 \{published data only\}}

Berlin I, Dautzenberg B, Lehmann B, Palmyre J, Liégey E, De Rycke Y, et al. Randomised, placebo-controlled, doubleblind, double-dummy, multicentre trial comparing electronic cigarettes with nicotine to varenicline and to electronic cigarettes without nicotine: the ECSMOKE trial protocol. BMJ Open 2019;9(5):e028832.

NCT03630614. Randomized trial of electronic cigarettes with or without nicotine in smoking cessation (ECSMOKE). clinicaltrials.gov/ct2/show/NCT03630614 (first received 15 August 2018).

\section{Caponnetto 2014 \{published data only\}}

* Caponnetto P, Polosa R, Auditore R, Minutolo G, Signorelli M, Maglia $\mathrm{M}$, et al. Smoking cessation and reduction in schizophrenia (SCARIS) with e-cigarette: study protocol for a randomized control trial. Trials [electronic resource] 2014;15:88.

NCT01979796. Antismoking effects of rlectronic cigarettes in subjects with schizophrenia and their potential influence on cognitive functioning: design of a randomized trial. Smoking Cessation And Reduction In Schizophrenia (The SCARIS Study). clinicaltrials.gov/show/NCT01979796 (accessed 16 July 2014).

\section{Caponnetto 2020 \{published data only\}}

Caponnetto P, Caruso M, Maglia M, Emma R, Saitta D, Busa B, et al. Non-inferiority trial comparing cigarette consumption, adoption rates, acceptability, tolerability, and tobacco harm reduction potential in smokers switching to Heated Tobacco Products or electronic cigarettes: Study protocol for a randomized controlled trial. Contemporary Clinical Trials Communications 2020;8:17.

\section{Fraser 2015 \{unpublished data only\}}

ACTRN12612001210864. An open-label randomised pragmatic policy trial examining effectiveness of short-term use of Nicotine Replacement Therapy (NRT) vs short- or long-term use of NRT vs short- or long-term use of NRT or electronic nicotine delivery systems for smoking cessation in cigarette smokers. ACTRN12612001210864 (accessed 15 August 2016).

* Fraser D, Borland R, Gartner C. Protocol for a randomised pragmatic policy trial of nicotine products for quitting or longterm substitution in smokers. BMC Public Health 2015;15:1026.

\section{ISRCTN13288677 \{published data only\}}

ISRCTN13288677. Can electronic cigarettes and nicotine replacement treatment help reduce smoking in smokers who struggle to quit? www.isrctn.com/ISRCTN13288677 (first received 17 March 2017).

ISRCTN61193406 \{published data only\}

ISRCTN61193406. Do e-cigarettes help smokers quit when not accompanied by intensive behavioural support? www.who.int/ trialsearch/Trial2.aspx?TrialID=ISRCTN611934062020 (first received 11 August 2020).

\section{Klonizakis 2017 \{published data only\}}

Klonizakis M, Crank H, Gumber A, Brose LS. Smokers making a quit attempt using e-cigarettes with or without nicotine or prescription nicotine replacement therapy: Impact on cardiovascular function (ISME-NRT) - a study protocol. BMC Public Health 2017;17(1):293.

NCT03061253. E-cigarettes and cardiovascular function (ISMENRT). clinicaltrials.gov/ct2/show/NCT03061253 (first received 23 February 2017).

\section{Murray 2020 \{published data only\}}

Murray RL, Brain K, Britton J, Quinn-Scoggins HD, Lewis S, McCutchan GM, et al. Yorkshire Enhanced Stop Smoking (YESS) study: a protocol for a randomised controlled trial to evaluate the effect of adding a personalised smoking cessation intervention to a lung cancer screening programme. BMJ Open 2020;10(9):e037086.

\section{NCT01842828 \{published data only\}}

NCT01842828. E-Cigarettes as an addition to multicomponent treatment for tobacco dependence: a pilot study. clinicaltrials.gov/show/NCT01842828 (accessed 16 July 2014).

\section{NCT01989923 \{published data only\}}

NCT01989923. Immediate smoking cessation for patients at risk for cervical dysplasia, cervical cancer and lower genital tract dysplasia and cancer - a feasibility study comparing nicotine replacement therapy with the electronic nicotine delivery system. clinicaltrials.gov/show/NCT01989923 (accessed 16 July 2014).

\section{NCT02004171 \{published data only\}}

NCT02004171. Electronic nicotine delivery devices (ENDDs) or nicotine inhaler for smoking cessation. clinicaltrials.gov/show/ NCT02004171 (accessed 16 July 2014).

NCT02124187 \{published data only\}

NCT02124187. Smoking cessation and reduction in depression (SCARID). clinicaltrials.gov/show/NCT02124187 (accessed 16 July 2014).

\section{NCT02398487 \{published data only\}}

NCT02398487. Head-to-head comparison of personal vaporizers versus cigalike: prospective 6-month randomized control design study. clinicaltrials.gov/show/NCT02398487 (accessed 17 February 2016).

\section{NCT02527980 \{published data only\}}

NCT02527980. E-cigarettes: dynamic patterns of use and health effects. clinicaltrials.gov/show/NCT02527980 (accessed 17 February 2016). 
NCT02590393 \{published data only\}

NCT02590393. The role of nicotine and non-nicotine alkaloids in e-cigarette use and dependence. clinicaltrials.gov/show/ NCT02590393 (accessed 17 February 2016).

\section{NCT02635620 \{published data only\}}

NCT02635620. Changes in lung function parameters, bronchial reactivity, state of health and smoking behaviour associated with changing from conventional smoking to electronic cigarettes. clinicaltrials.gov/show/NCT02635620 (accessed 17 February 2016).

\section{NCT03589989 \{published data only\}}

NCT03589989. The ESTxENDS Trial- Electronic Nicotine Delivery Systems (ENDS/Vaporizer/E-cigarette) as an aid for smoking cessation. (ESTxENDS). clinicaltrials.gov/ct2/show/record/ NCT03589989 (first received 18 July 2018).

NCT03603340. The ESTxENDS Trial- Effects of using Electronic Nicotine Delivery Systems (ENDS/Vaporizer/E-cig) on depression (ESTxENDS). clinicaltrials.gov/show/NCT03603340 (first received 27 July 2018).

NCT03603353. The ESTxENDS Trial- Effects of using Electronic Nicotine Delivery Systems (ENDS/Vaporizer/E-cig) on sleep quality. (ESTxENDS). clinicaltrials.gov/show/NCT03603353 (first received 27 July 2018).

NCT03612336. The ESTxENDS Trial- Metabolic effects of using Electronic Nicotine Delivery Systems (ENDS/Vaporizer/Ecig) (ESTxENDS). clinicaltrials.gov/show/NCT03612336 (first received 2 August 2018).

NCT03612375. ESTxENDS Trial-Oxidative stress induced by Electronic Nicotine Delivery Systems (ENDS/Vaporizer/Ecig) measured in urine (ESTxENDS). clinicaltrials.gov/show/ NCT03612375 (first received 2 August 2018).

NCT03612453. ESTxENDS Trial- Oxidative stress induced by Electronic Nicotine Delivery Systems (ENDS/Vaporizer/Ecig) measured in EBC (ESTxENDS). clinicaltrials.gov/show/ NCT03612453 (first received 2 August 2018).

NCT03612544. The ESTxENDS Trial- Toxins from using Electronic Nicotine Delivery Systems (ENDS/Vaporizer/E-cig) (ESTxENDS). clinicaltrials.gov/show/NCT03612544 (first received 2 August 2018).

NCT03632421. The ESTxENDS Trial-effects of using Electronic Nicotine Delivery Systems (ENDS/Vaporizer/E-cig) on respiratory symptoms (ESTxENDS). clinicaltrials.gov/show/ NCT03632421 (first received 15 August 2018).

NCT03938298. The ESTxENDS Trial: Pulmonary function substudy (PulmENDS). clinicaltrials.gov/ct2/show/nct03938298 (first received 6 May 2019).

NCT04236791. The ESTxENDS Trial- Electronic Nicotine Delivery Systems as an aid for smoking cessation-extension of followup. clinicaltrials.gov/show/NCT04236791 2020 (first received 22 January 2020).
NCT04244773. ESTxENDS Trial: MN Substudy - Micronuclei in buccal epithelium, a surrogate measure of future cancer risk, induced by Electronic Nicotine Delivery Systems (ENDS/ Vaporizer/E-cig). clinicaltrials.gov/show/NCT04244773 (first receved 28 January 2020).

NCT04617444. The ESTxENDS Trial- Effects of using Electronic Nicotine Delivery Systems (ENDS/Vaporizer/E-cig) on olfactory function. clinicaltrials.gov/show/NCT04617444 (first received 05 November 2020).

\section{NCT03700112 \{published data only\}}

NCT03700112. Clinical study comparing 7 ENDS products and 1 combustible cigarette using 2 delivery methods. clinicaltrials.gov/ct2/show/NCT03700112 (first received 7 December 2018).

\section{NCT03962660 \{published data only\}}

NCT03962660. Harm Reduction for Tobacco Smoking with support of Tobacco-Rreplacing Electronic Nicotine Delivery Systems (HaRTS-TRENDS). clinicaltrials.gov/ct2/show/record/ NCT03962660 (first received 24 May 2019).

\section{NCT04063267 \{published data only\}}

NCT04063267. Electronic cigarettes as a harm reduction strategy in individuals with substance use disorder. clinicaltrials.gov/ct2/show/NCT04063267 (first received 21 August 2019).

\section{NCT04231838 \{published data only\}}

NCT04231838. Metabolic syndrome in diabetic smokers using cigarettes \& combustion-free nicotine delivery systems. clinicaltrials.gov/show/NCT04231838 (first received 18 January 2020).

NCT04238832 \{published data only\}

NCT04238832. Salt-based e-cigarette and IQOS Study. clinicaltrials.gov/show/NCT04238832 (firs treceived 18 January 2020).

NCT04452175 \{published data only\}

NCT04452175. Cigarette consumption after switching to high or low nicotine strENght E-cigaretteS In Smokers With Schizophrenia (GENESIS). clinicaltrials.gov/show/NCT04452175 2020 (first received 30 June 2020).

\section{NCT04649645 \{published data only\}}

NCT04649645. International randomized controlled tria evaluating changes in oral health in smokers after switching to combustion-free nicotine delivery systems. clinicaltrials.gov/ show/NCT04649645 (first received 02 December 020).

\section{Additional references}

\section{Akiyama 2021}

Akiyama Y, Sherwood N. Systematic review of biomarker findings from clinical studies of electronic cigarettes and heated tobacco products. Toxicology Reports 2021;8:282-94. 


\section{Amato 2020}

Amato L, Cruciani F, Solimini R, Barca A, Pacifici R, Davoli M. Effects of electronic cigarettes on health: a systematic review of the available evidence. Recenti Progressi in Medicina 2020;111(1):30-43.

\section{ASH 2019}

Action on Smoking and Health. Use of electronic cigarettes (vapourisers) among adults in Great Britain. ash.org.uk/ information-and-resources/fact-sheets/statistical/use-of-ecigarettes-among-adults-in-great-britain-2019/ (accessed 21 July 2020).

\section{Balfour 2004}

Balfour D. The neurobiology of tobacco dependence: a preclinical perspective on the role of dopamine projections to the nucleus. Nicotine \& Tobacco Research 2004;6(6):899-912.

\section{Barbeau 2013}

Barbeau AM, Burda J, Siegel M. Perceived efficacy of e-cigarettes versus nicotine replacement therapy among successful ecigarette users: a qualitative approach. Addiction: Science \& Clinical Practice 2013;8(1):5.

\section{Barrett 2010}

Barrett SP. The effects of nicotine, denicotinized tobacco, and nicotine-containing tobacco on cigarette craving, withdrawal, and self-administration in male and female smokers. Behavorial Pharmacology 2010;21(2):144-52.

\section{Benowitz 2010}

Benowitz NL. Nicotine addiction. New England Journal of Medicine 2010;362(24):2295-303.

\section{Besaratinia 2019}

Besaratinia A, Tommasi S. Vaping: a growing global health concern. EClinicalMedicine 2019;17:100208.

\section{Blount 2020}

Blount BC, Karwowski MP, Shields PG, Morel-Espinosa M, Valentin-Blasini L, Gardner M, et al. Vitamin E acetate in bronchoalveolar-lavage fluid associated with EVALI. New England Journal of Medicine 2020;382(8):697-705.

\section{Brooker 2019}

Brooker J, Synnot A, McDonald S, Elliott J, Turner T, et al. Guidance for the production and publication of Cochrane living systematic reviews: Cochrane Reviews in living mode;December 2019. community.cochrane.org/sites/default/files/uploads/ inline-files/Transform/201912_LSR_Revised_Guidance.pdf (accessed 30 July 2020).

\section{Cahill 2016}

Cahill K, Lindson-Hawley N, Thomas K, Fanshawe TR, Lancaster T. Nicotine receptor partial agonists for smoking cessation. Cochrane Database of Systematic Reviews 2016, Issue 5. Art. No: CD006103. [DOI: 10.1002/14651858.CD006103.pub7] vaping, products. www.cdc.gov/tobacco/basic_information/ e-cigarettes/severe-lung-disease.html\#latest-outbreakinformation Feb 2020 (accessed 12 Aug 2020).

\section{Chen 2016}

Chen C, Zhuang YL, Zhu SH. E-cigarette design preference and smoking cessation: a U.S. population study. American Journal of Preventive Medicine 2016;51(3):356-63. [DOI: 10.1016/ j.amepre.2016.02.002]

\section{Covidence [Computer program]}

Veritas Health Innovation Covidence. Version accessed 30 July 2020. Melbourne, Australia: Veritas Health Innovation. Available at covidence.org.

\section{Cox 2017}

Cox S, Jakes S. Nicotine and e-cigarettes: rethinking addiction in the context of reduced harm. International Journal of Drug Policy 2017;44:84-5.

\section{Cox 2019b}

Cox S, Leigh NJ, Vanderbush TS, Choo E, Goniewicz ML, Dawkins L. An exploration into "do-it-yourself" (DIY) e-liquid mixing: sers' motivations, practices and product laboratory analysis. Addictive Behaviors Reports 2019;9:100151.

\section{Dawkins 2013b}

Dawkins L, Turner J, Roberts A, Soar K. 'Vaping' profiles and preferences: an online survey of electronic cigarette users. Addiction 2013;108(6):1115-25.

\section{Dawkins 2016}

Dawkins LE, Kimber CF, Doig M, Feyerabend C, Corcoran O. Self-titration by experienced e-cigarette users: blood nicotine delivery and subjective effects. Psychopharmacology (Berl) 2016;233(14-16):2933-41. [DOI: 10.1007/s00213-016-4338-2]

\section{Dawkins 2018}

Dawkins L, Cox S, Goniewicz M, McRobbie H, Kimber C, Doig M, et al. 'Real-world' compensatory behaviour with low nicotine concentration e-liquid: subjective effects and nicotine, acrolein and formaldehyde exposure. Addiction 2018;113(10):1874-82.

\section{Donny 2007}

Donny EC, Houtsmuller E, Stitzer ML. Smoking in the absence of nicotine: behavioral, subjective and physiological effects over 11 days. Addiction 2007;102(2):324-34.

\section{Donny 2009}

Donny EC, Jones M. Prolonged exposure to denicotinized cigarettes with or without transdermal nicotine. Drug and Alcohol Dependence 2009;104(1-2):23-33.

\section{Donny 2015}

Donny EC, Denlinger RL, Tidey JW, Koopmeiners JS, Benowitz NL, Vandrey RG, et al. Randomized trial of reducednicotine standards for cigarettes. New England Journal of Medicine 2015;373(14):1340-9.

\section{CDC 2020}

Centres for Disease Control and Prevention. Outbreak of lung injury associated with the use of e-cigarette, or 


\section{Du 2020}

Du Y, Liu B, Xu G, Rong S, Sun Y, Wu Y, et al. Association of electronic cigarette regulations with electronic cigarette use among adults in the United States. JAMA Network Open 2020;3(1):e1920255.

\section{E-cigarette ontology 2021}

E-cigarette v1. Qeios. [URL: www.qeios.com/read/0WX80U]

\section{Etter 2011}

Etter JF, Bullen C. Electronic cigarette: users profile, utilization, satisfaction and perceived efficacy. Addiction 2011;106(11):2017-28

\section{European Parliament 2014}

European Parliament and Council of the European Union. Directive 2014/40/EU of the European Parliament and of the Council of 3 April 2014 on the approximation of the laws, regulations and administrative provisions of the Member States concerning the manufacture, presentation and sale of tobacco and related products and repealing Directive 2001/37/EC. 2014 (accessed 25th November 2014).

\section{Fagerström 2004}

Fagerström KO, Hughes JR. Nicotine concentrations with concurrent use of cigarettes and nicotine replacement: a review. Nicotine \& Tobacco Research 2004;4(Suppl 2):S73-9.

\section{Farsalinos 2014}

Farsalinos KE, Polosa R. Safety evaluation and risk assessment of electronic cigarettes as tobacco cigarette substitutes: a systematic review. Therapeutic Advances in Drug Safety 2014;5(2):67-86.

\section{Goniewicz 2012}

Goniewicz ML, Kuma T, Gawron M, Knysak J, Kosmider L. Nicotine levels in electronic cigarettes. Nicotine \& Tobacco Research 2012;15(1):158-66.

\section{Goniewicz 2014}

Goniewicz ML, Hajek P, McRobbie H. Nicotine content of electronic cigarettes, its release in vapour and its consistency across batches: regulatory implications. Addiction 2014;109(3):500-7.

\section{GRADEpro GDT [Computer program]}

McMaster University (developed by Evidence Prime) GRADEpro GDT. Version accessed 30 July 2020. Hamilton (ON): McMaster University (developed by Evidence Prime). Available at gradepro.org.

\section{Hajek 1999}

Hajek P, West R, Foulds J, Nilsson F, Burrows S, Meadow A. Randomized comparative trial of nicotine polacrilex, a transdermal patch, nasal spray, and an inhaler. Archives of Internal Medicine 1999;159(17):2033-8.

\section{Hajek 2014}

Hajek P, Etter J-F, Benowitz N, Eissenberg T, McRobbie H. Electronic cigarettes: review of use, content, safety, effects on smokers and potential for harm and benefit. Addiction 2014;109(11):1801-10.

\section{Hajek 2015b}

Hajek P, Goniewicz ML, Phillips A, Myers Smith K, West O, McRobbie H. Nicotine intake from electronic cigarettes on initial use and after 4 weeks of regular use. Nicotine \& Tobacco Research 2015;17(2):175-9.

\section{Hajek 2017}

Hajek P, Przulj D, Phillips A, Anderson R, McRobbie H. Nicotine delivery to users from cigarettes and from different types of ecigarettes. Psychopharmacology (Berl) 2017;234(5):773-9.

\section{Hajek 2020}

Hajek P, Pittaccio K, Pesola F, Myers Smith K, Phillips-Waller A, Przulj D. Nicotine delivery and users' reactions to Juul compared with cigarettes and other e-cigarette products. Addiction 2020;115(6):1141-8.

\section{Hall 2020}

Hall W, Gartner C, Bonevski B. Lessons from the public health responses to the US outbreak of vaping-related lung injury. Addiction 2020 [Epub ahead of print].

\section{Hammond 2020}

Hammond D, Rynard VL, Reid JL. Changes in prevalence of vaping among youths in the United States, Canada, and England from 2017 to 2019. JAMA Pediatrics 2020;174(8):797-800.

\section{Hartmann-Boyce 2018a}

Hartmann-Boyce J, Chepkin SC, Ye W, Bullen C, Lancaster T. Nicotine replacement therapy versus control for smoking cessation. Cochrane Database of Systematic Reviews 2018, Issue 5. Art. No: CD000146. [DOI: 10.1002/14651858.CD000146.pub5]

\section{Hartmann-Boyce 2018b}

Hartmann-Boyce J, Fanshawe TR, Lindson N, LivingstoneBanks J, Ordonez-Mena J, Aveyard P. Behavioural interventions for smoking cessation: an overview and network meta-analysis. Cochrane Database of Systematic Reviews 2018, Issue 12. Art. No: CD013229. [DOI: 10.1002/14651858.CD013229]

\section{Hartmann-Boyce 2019}

Hartmann-Boyce J, Hong B, Livingstone-Banks J, Wheat $\mathrm{H}$, Fanshawe TR. Additional behavioural support as an adjunct to pharmacotherapy for smoking cessation. Cochrane Database of Systematic Reviews 2019, Issue 6. Art. No: CD009670. [DOI: 10.1002/14651858.CD009670.pub4]

\section{Hartnett 2020}

Hartnett KP, Kite-Powell A, Patel MT, Haag BL, Sheppard MJ, Dias TP, et al. Syndromic surveillance for e-cigarette, or vaping, product use-associated lung injury. New England Journal of Medicine 2020;382(8):766-72.

\section{Higgins 2003}

Higgins JP, Thompson SG, Deeks JJ, Altman DG. Measuring inconsistency in meta-analyses. BMJ 2003;327(7414):557-60. 


\section{Higgins 2011}

Higgins JP, Green S (editors). Cochrane Handbook for Systematic Reviews of Interventions Version 5.1.0 (updated March 2011). The Cochrane Collaboration, 2011. Available from www.cochrane-handbook.org.

\section{Higgins 2021}

Higgins JP, Thomas J, Chandler J, Cumpston M, Li T, Page MJ, et al (editors). Cochrane Handbook for Systematic Reviews of Interventions version 6.2 (updated February 2021). The Cochrane Collaboration, 2021. Available from www.training.cochrane.org/handbook.

\section{Hitchman 2015}

Hitchman SC, Brose LS, Brown J, Robson D, McNeill A. Associations between e-cigarette type, frequency of use, and quitting smoking: findings from a longitudinal online panel survey in Great Britain. Nicotine \& Tobacco Research 2015;17(10):1187-94.

\section{Huang 2019}

Huang J, Duan Z, Kwok J, Binns S, Vera LE, Kim Y, et al. Vaping versus JUULing: how the extraordinary growth and marketing of JUUL transformed the US retail e-cigarette market. Tobacco Control 2019;28(2):146-51.

\section{Hughes 2004}

Hughes JR, Keely J, Naud S. Shape of the relapse curve and long-term abstinence among untreated smokers. Addiction 2004;99(1):29-38.

\section{Jiang 2016}

Jiang N, Chen J, Wang MP, McGhee SM, Kwong AC, Lai VW, et al. Electronic cigarette awareness and use among adults in Hong Kong. Addictive Behaviors 2016;52:34-8.

\section{Levin 1993}

Levin ED, Behm F, Carnahan E, LeClair R, Shipley R, Rose JE. Clinical trials using ascorbic acid aerosol to aid smoking cessation. Drug and Alcohol Dependence 1993;33(3):211-23.

\section{Lindson 2019}

Lindson N, Chepkin SC, Ye W, Fanshawe TR, Bullen C, HartmannBoyce J. Different doses, durations and modes of delivery of nicotine replacement therapy for smoking cessation. Cochrane Database of Systematic Reviews 2019, Issue 4. Art. No: CD013308. [DOI: 10.1002/14651858.CD013308]

\section{Martinez-Morata 2021}

Martinez-Morata I, Sanchez TR, Shimbo D, Navas-Acien A. Electronic cigarette use and blood pressure endpoints: a systematic review. Current Hypertension Reports 2021;23(1):1.

\section{McDonald 2020}

McDonald CF, Jones S, Beckert L, Bonevski B, Buchanan T, Bozier J, et al. Electronic cigarettes: a position statement from the Thoracic Society of Australia and New Zealand. Respirology 2020 [Epub ahead of print].

\section{McNeill 2021}

McNeill A, Brose LS, Calder R, Bauld L, Robson D. Vaping in England: an evidence update including vaping for smoking cessation, February 2021: a report commissioned by Public Health England London, UK: Public Health England. assets.publishing.service.gov.uk/government/ uploads/system/uploads/attachment_data/file/962221/ Vaping_in_England_evidence_update_February_2021.pdf 2021.

\section{McRobbie 2016}

McRobbie H, Przulj D, Smith KM, Cornwall D. Complementing the standard multicomponent treatment for smokers with denicotinized cigarettes: a randomized trial. Nicotine \& Tobacco Research 2016;18(5):1134-41.

\section{NASEM 2018}

National Academies of Sciences, Engineering, and Medicine, Health and Medicine Division, Board on Population Health and Public Health Practice. Public Health Consequences of ECigarettes. Washington (DC): National Academies Press (US), 2018.

\section{Notley 2018a}

Notley C, Colllins R. Redefining smoking relapse as recovered social identity - secondary qualitative analysis of relapse narratives. Journal of Substance Use 2018;23(6):660-6.

\section{Notley 2018b}

Notley C, Ward E, Dawkins L, Holland R. The unique contribution of e-cigarettes for tobacco harm reduction in supporting smoking relapse prevention. Harm Reduct $J$ 2018;15(1):31.

\section{Palipudi 2016}

Palipudi KM, Mbulo L, Morton J, Bunnell R, Blutcher-Nelson G, Kosen S, et al. Awareness and current use of electronic cigarettes in Indonesia, Malaysia, Qatar, and Greece: findings from 2011-2013 global adult tobacco surveys. Nicotine \& Tobacco Research 2016;18(4):501-7.

\section{Patnode 2021}

Patnode CD, Henderson JT, Coppola EL, Melnikow J, Durbin S, Thomas RG. Interventions for tobacco cessation in adults, including pregnant persons: updated evidence report and systematic review for the US Preventive Services Task Force. JAMA 2021;325(3):280-98.

\section{Perkins 2010}

Perkins KA, Karelitz JL, Conklin CA, Sayette MA, Giedgowd GE. Acute negative affect relief from smoking depends on the affect situation and measure but not on nicotine. Biological Psychology 2010;67(8):707-14.

\section{Pickworth 1999}

Pickworth WB, Fant RV, Nelson RA, Rohrer MS, Henningfield JE. Pharmacodynamic effects of new de-nicotinized cigarettes. Nicotine \& Tobacco Research 1999;1(4):357-64. 


\section{Przulj 2013}

Przulj D, McRobbie H, Hajek P. The effect of sensorimotor replacement on smoking cessation and craving. Open Addiction Journal 2013;5:41-50.

\section{RCP 2016}

Tobacco Advisory Group of the Royal College of Physicians. Nicotine Without Smoke-Tobacco Harm Reduction. London: Royal College of Physicians, 2016.

\section{Review Manager 2020 [Computer program]}

Nordic Cochrane Centre, The Cochrane Collaboration Review Manager 5 (RevMan 5). Version 5.4. Copenhagen: Nordic Cochrane Centre, The Cochrane Collaboration, 2020.

\section{Romeu 2020}

Romeu ER. Voke ${ }^{\circledR}$ nicotine inhaler for smoking cessation and reduction. ntag.nhs.uk/docs/app/NTAG-Apprasial-Vokenicotine-inhalator-Feb-2020-final-for-web.pdf 2020.

\section{Rose 1985}

Rose JE, Tashkin DP, Ertle A, Zinser MC, Lafer R. Sensory blockade of smoking satisfaction. Pharmacology Biochemistry \& Behavior 1985;23(2):289-93.

\section{Rose 1993}

Rose JE, Behm FM, Levin ED.

Role of nicotine dose and sensory cues in the regulation of smoke intake. Pharmacology

Biochemistry \& Behavior 1993;44(4):891-900.

\section{Rose 1994}

Rose JE, Behm FM. Inhalation of vapor from black pepper extract reduces smoking withdrawal symptoms. Drug and Alcohol Dependence 1994;34(3):225-9.

\section{Rose $\mathbf{2 0 0 0}$}

Rose JE, Behm FM, Westman EC, Johnson M. Dissociating nicotine and nonnicotine components of cigarette smoking. Pharmacology Biochemistry \& Behavior 2000;67(1):71-81.

\section{Rose 2006}

Rose JE. Nicotine and nonnicotine factors in cigarette addiction. Psychopharmacology 2006;184(3-4):274-85.

\section{Russell 1990}

Russell MA. Nicotine intake and its control over smoking. In: Wonnacott S, Russell MA, Stolerman I, editors(s). Nicotine Psychopharmacology: Molecular, Cellular and Behavioural Aspects. London: Oxford University Press, 1990:374-418.

\section{Schneider 2001}

Schneider NG, Olmstead RE, Franzon MA, Lunell E. The nicotine inhaler: clinical pharmacokinetics and comparison with other nicotine treatments. Clinical Pharmcokinetics 2001;40(9):661-84.

\section{Smets 2019}

Smets J, Baeyens F, Chaumont M, Adriaens K, Van Gucht D. When less is more: vaping low-nicotine vs. high-nicotine eliquid is compensated by increased wattage and higher liquid consumption. International Journal of Environmental Research and Public Health 2019;16(5):723.

\section{Talih 2020}

Talih S, Salman R, El-Hage R, Karam E, Salam S, Karaoghlanian N, et al. A comparison of the electrical characteristics, liquid composition, and toxicant emissions of JUUL USA and JUUL UK e-cigarettes. Scientific Reports 2020;10(1):7322.

\section{Tattan-Birch 2020}

Tattan-Birch H, Brown J, Shahab L, Jackson SE. Association of the US outbreak of vaping-associated lung injury with perceived harm of e-cigarettes compared with cigarettes. JAMA Network Open 2020;3(6):e206981.

\section{USPFTS 2021}

Interventions for Tobacco Smoking Cessation in Adults, Including Pregnant Persons. U.S. Preventive Services Task Force 2021:www.uspreventiveservicestaskforce.org/uspstf/ recommendation/tobacco-use-in-adults-and-pregnant-womencounseling-and-interventions.

\section{Walker 2012}

Walker N, Howe C, Bullen C, Grigg M, Glover M, McRobbie H, et al. The combined effect of very low nicotine content cigarettes, used as an adjunct to usual Quitline care (nicotine replacement therapy and behavioural support), on smoking cessation: a randomized controlled trial. Addiction 2012;107(10):1857-67.

\section{Wang 2021}

Wang RJ, Bhadriraju S, Glantz SA. E-Cigarette Use and Adult Cigarette Smoking Cessation: A Meta-Analysis. American Journal of Public Health 2021;111:230-46.

\section{Westman 1995}

Westman EC, Behm FM, Rose JE. Airway sensory replacement combined with nicotine replacement for smoking cessation. A randomized, placebo-controlled trial using a citric acid inhaler. Chest 1995;107(5):1358-64.

\section{Westman 1996}

Westman EC, Behm FM, Rose JE. Dissociating the nicotine and airway sensory effects of smoking. Pharmacology Biochemistry \& Behavior 1996;53(2):309-15.

\section{Yingst 2019a}

Yingst JM, Foulds J, Veldheer S, Hrabovsky S, Trushin N, Eissenberg TT, et al. Nicotine absorption during electronic cigarette use among regular users. PLOS One 2019;14(7):e0220300.

\section{Yingst 2019b}

Yingst JM, Hrabovsky S, Hobkirk A, Trushin N, Richie JP Jr, Foulds J. Nicotine absorption profile among regular users of a pod-based electronic nicotine delivery system. JAMA Network Open 2019;2(11):e1915494.

\section{Zhang 2021}

Zhang YY, Bu FL, Dong F, Wang JH, Zhu SJ, Zhang XW, et al. The effect of e-cigarettes on smoking cessation and cigarette 
smoking initiation: an evidence-based rapid review and metaanalysis. Tobacco Induced Diseases 2021;19(4). [DOI: 10.18332/ tid/131624]

\section{References to other published versions of this review Hartmann-Boyce 2016}

Hartmann-Boyce J, McRobbie H, Bullen C, Begh R, Stead LF, Hajek P. Electronic cigarettes for smoking cessation. Cochrane Database of Systematic Reviews 2016, Issue 9. Art. No: CD010216. [DOI: 10.1002/14651858.CD010216.pub3]

\section{Hartmann-Boyce 2020}

Hartmann-Boyce J, McRobbie H, Lindson N, Bullen C, Begh R, Theodoulou A, et al. Electronic cigarettes for smoking cessation.

\section{CHARACTERISTICS OF STUDIES}

Characteristics of included studies [ordered by study ID]
Cochrane Database of Systematic Reviews 2020, Issue 10. Art. No: CD010216. [DOI: 10.1002/14651858.CD010216.pub4]

\section{McRobbie 2012}

McRobbie H, Bullen C, Hajek P. Electronic cigarettes for smoking cessation and reduction. Cochrane Database of Systematic Reviews 2012, Issue 11. Art. No: CD010216. [DOI: 10.1002/14651858.CD010216]

\section{McRobbie 2014}

McRobbie H, Bullen C, Hartmann-Boyce J, Hajek P. Electronic cigarettes for smoking cessation and reduction. Cochrane Database of Systematic Reviews 2014, Issue 12. Art. No: CD010216. [DOI: 10.1002/14651858.CD010216.pub2]

* Indicates the major publication for the study

Adriaens 2014

\section{Study characteristics}

Methods

Design: 3-armed RCT; with all participants then assigned to nicotine EC (treated as cohort in this review)

Recruitment: Advertisement on university website, flyers on university campuses, emails to personnel and advertisement in local newspaper

Setting: Community and laboratory, Belgium

Study start date/end date: Not stated

Participants

Total N: 48 provided data

Randomized to: EC1 16; EC2 17; control 17

Inclusion criteria:

- Smoker for at least 3 years,

- Smoking at least $10 \mathrm{cpd}$, not intending to quit in the near future but willing to try a less unhealthy alternative

Exclusion criteria:

- Diabetes;

- Severe allergies;

- Asthma or other respiratory diseases; psychiatric problems;

- Dependence on chemicals other than nicotine;

- Pregnancy;

- Breastfeeding;

- Hypertension;

- CV disease;

- Currently using any kind of smoking cessation therapy; prior use of EC

$56 \%$ women, mean age 44 , mean cpd 19, mean FTCD 5.79, all unwilling to quit with no baseline EC use 
Intervention: 2 intervention groups (EC1 and EC2) provided with $E C$ and instructed to use $E C$ or smoke ad libitum (EC1 group provided with Joyetech eGO-C, EC2 group provided with Kanger T2-CC) and provided guidance on EC use. For both types, provided $30 \mathrm{~mL}$ bottles of tobacco-flavored e-liquid (Dekang "Turkish Blend"), containing $18 \mathrm{mg} / \mathrm{mL}$ of nicotine. 4 bottles at baseline replenished at 4 weeks, keep any remaining after 8 weeks

Control: 6 bottles for 2 months at week 8 (half offered EC1, half offered EC2); no guidance on use

\begin{tabular}{|c|c|}
\hline \multirow[t]{3}{*}{ Outcomes } & $\begin{array}{l}3 \text { lab sessions over } 2 \text { months (weeks } 1,4 \text { and } 8 \text { ), plus online questionnaires, further follow-up at } 3 \text { and } 6 \\
m \text { after last lab session }\end{array}$ \\
\hline & Cessation: measured but definition not provided, validated with eCO $5 \mathrm{ppm}$ or less \\
\hline & $\begin{array}{l}\text { Adverse events and biomarkers: eCO, salivary cotinine measured during lab sessions. Also collected } \\
\text { craving and withdrawal symptoms via lab sessions, "benefits and complaints", mood, EC usage }\end{array}$ \\
\hline Study funding & $\begin{array}{l}\text { "No external funding for this study was obtained. Electronic cigarettes and e-liquids were purchased at } \\
\text { E-cig4U ( } t \text { Rond 10, } 4285 \text { DE Woudrichem, The Netherlands; http://www.e-cig4u.nl/) with balances of } \\
\text { previous research funds obtained by Frank Baeyens." }\end{array}$ \\
\hline Author declarations & The authors declare no conflict of interest \\
\hline \multirow[t]{2}{*}{ Notes } & Randomization was for short-term outcomes only \\
\hline & Additional data provided from authors \\
\hline
\end{tabular}

\section{Risk of bias}

\begin{tabular}{|c|c|c|}
\hline Bias & Authors' judgement & Support for judgement \\
\hline $\begin{array}{l}\text { Random sequence genera- } \\
\text { tion (selection bias) }\end{array}$ & Low risk & $\begin{array}{l}\text { Block randomization was performed by using a randomization tool available } \\
\text { on the website www.randomizer.org }\end{array}$ \\
\hline $\begin{array}{l}\text { Allocation concealment } \\
\text { (selection bias) }\end{array}$ & Unclear risk & Not specified \\
\hline $\begin{array}{l}\text { Blinding of participants } \\
\text { and personnel (perfor- } \\
\text { mance bias) } \\
\text { All outcomes }\end{array}$ & Low risk & $\begin{array}{l}\text { Unblinded but as this review only includes data on objective measurements } \\
\text { and not cessation judged unlikely to affect outcomes }\end{array}$ \\
\hline $\begin{array}{l}\text { Blinding of outcome as- } \\
\text { sessment (detection bias) } \\
\text { All outcomes }\end{array}$ & Low risk & $\begin{array}{l}\text { Unblinded but as this review only includes data on objective measurements } \\
\text { and not cessation judged unlikely to affect outcomes }\end{array}$ \\
\hline $\begin{array}{l}\text { Incomplete outcome data } \\
\text { (attrition bias) } \\
\text { All outcomes }\end{array}$ & Low risk & $\begin{array}{l}36 \text { out of } 48 \text { completed follow-up (11/16 in EC1 group, } 12 / 17 \text { in EC2 group, } \\
13 / 17 \text { in control group) }\end{array}$ \\
\hline $\begin{array}{l}\text { Selective reporting (re- } \\
\text { porting bias) }\end{array}$ & Unclear risk & $\begin{array}{l}\text { Outcome reporting somewhat non-traditional; for example, collecting com- } \\
\text { plaints but not explicitly adverse events, and incidence of AEs not reported. } \\
\text { Unable to find prospectively-registered protocol }\end{array}$ \\
\hline
\end{tabular}

Baldassarri 2018

\section{Study characteristics}


Baldassarri 2018 (Continued)

Methods
Design: Randomized parallel-assignment double-blind trial

Recruitment: outpatient pulmonary and primary care clinics, Tobacco Treatment Service, referrals from medical providers

Setting: Hospital outpatient and primary care clinics, USA

Study start date: October 2014; Study end date: June 2014
Total N: 40

N per arm: Non-Nicotine: 20; Nicotine EC: 20

Inclusion criteria:

- Age 18 years or older

- Smoking 1 or more cpd

- Willing to quit smoking

Exclusion criteria:

- Unstable psychiatric or medical conditions requiring hospitalization within the past 4 months;

- Acute coronary syndromes or stroke within the past 30 days;

- History of allergic reactions to adhesives;

- Women who were pregnant, nursing, or not practicing effective contraception;

- Current use of an EC for the purpose of stopping tobacco cigarette smoking

Women: 52.5\%; Mean age: 53 Mean cpd: 17 Mean FTND: 5.9; motivated to quit

E cigarette use at baseline: Not reported
Interventions

\section{EC: Refillable}

Both groups received standard care (8 weeks nicotine patch and counseling) and were randomized to nicotine EC or non-nicotine EC.

EC: eGO style EC ( 650 mAh battery, EVOD clearomizer, $3.7 \mathrm{~V}, 1.8 \Omega$ single bottom coil), provided with eliquid purchased from an online vape shop $(0 \mathrm{mg} / \mathrm{ml}$ or $24 \mathrm{mg} / \mathrm{ml}$ nicotine strength, 70/30 propylene glycol/vegetable glycerin, tobacco flavor); Instructed to use it as needed as a substitute for tobacco to try to satisfy cravings to smoke. If the patch alone proved adequate to prevent withdrawal and smoking cravings, the participant was advised not to use the EC. Additional EC devices, replacement coils, and liquid were provided as needed for the first 8 weeks of the study

Outcomes Questionnaires and CO measurements taken at baseline, treatment visits at week 2, 4, 6, 8 and follow-up at week 24

Cessation: 7-day point prevalence abstinence, eCO $\leq 6 \mathrm{ppm}$

Adverse events and biomarkers: Side effects were measured although it is unclear whether a questionnaire with prespecified symptoms was used

Spirometry and FeNO at baseline and 6-month follow-up

Other outcomes: Change in reported number of cpd at weeks 8 and 24; Change in per cent predicted FEV1 and FVC from baseline to week 24, and EC use patterns

Study funding
"Funding for this study was provided by the Yale School of Medicine, Section of Pulmonary, Critical Care, and Sleep Medicine and the National Heart, Lung, and Blood Institute grant T32HL007778. NHLBI had no role in the study design, collection, analysis, or interpretation of the data, writing the manuscript, or the decision to submit the paper for publication." 
Baldassarri 2018 (Continued)

Author declarations
"Dr. Toll received a grant from Pfizer for medicine only for a research study, and he receives funding as an expert witness in litigation filed against the tobacco industry. Dr. Chupp received grants from NIH, Genetech, Glaxo Smith Kline, Astra Zeneca/Medimmune and Boston Scientific. He received consulting/speaking fees from Genetech, Astra Zeneca/Medimmune, Mannkind, and Boston Scientific. There are no other conflicts of interest for the remaining authors."

Notes

New for 2020 update. Study listed as ongoing study NCT02498145 in 2016 review update

Additional data provided from authors

\section{Risk of bias}

\begin{tabular}{lll}
\hline Bias & Authors' judgement & Support for judgement \\
\hline $\begin{array}{l}\text { Random sequence genera- } \\
\text { tion (selection bias) }\end{array}$ & Low risk & $\begin{array}{l}\text { Quote: "Participants were randomized using a random number generator with } \\
1: 1 \text { blocked randomization (block size n= 8)." }\end{array}$ \\
\hline $\begin{array}{l}\text { Allocation concealment } \\
\text { (selection bias) }\end{array}$ & Unclear risk & $\begin{array}{l}\text { Both groups received standard care (nicotine patch and counseling) and were } \\
\text { randomized to: nicotine EC or non-nicotine EC (no further detail given) }\end{array}$ \\
\hline $\begin{array}{l}\text { Blinding of participants } \\
\begin{array}{l}\text { and personnel (perfor- } \\
\text { mance bias) }\end{array}\end{array}$ & Low risk & $\begin{array}{l}\text { Quote: "Treatment assignment was blinded to both the investigators and par- } \\
\text { ticipants" }\end{array}$ \\
All outcomes & \\
\hline
\end{tabular}

Blinding of outcome as- Low risk CO biochemically validated

sessment (detection bias)

All outcomes

$\begin{array}{lll}\begin{array}{l}\text { Incomplete outcome data } \\ \text { (attrition bias) } \\ \text { All outcomes }\end{array} & \text { High risk } & \text { Quote: “The study had a modest loss to follow-up (20\%) at week 24." } \\ & \text { Number lost to follow-up in each group is not reported in the paper } \\ & \begin{array}{l}\text { Week } 24 \text { retention rate: Nicotine EC group: } 19 / 20 \text { (95\%); Non-nicotine EC } \\ \text { group: } 13 / 20(65 \%) ;>20 \% \text { difference between groups }\end{array} \\ \begin{array}{l}\text { Selective reporting (re- } \\ \text { porting bias) }\end{array} & \text { Low risk } & \begin{array}{l}\text { Outcomes reported align with those listed in the clinicaltrials.gov record. (reg- } \\ \text { istered 2015; prior to study completion in 2016) }\end{array}\end{array}$

\section{Bell 2017}

\section{Study characteristics}

\begin{tabular}{ll} 
Methods & Design: Pragmatic, uncontrolled, mixed-methods trial \\
& Recruitment: Targeted settings for people with HIV \\
& Setting: Community, Brisbane, Australia \\
& Study start date: 21 February 2017; Study end date: 26 October 2017 \\
\hline Participants & Total N: 30 \\
Inclusion criteria: \\
- Diagnosis of HIV \\
- Aged 18 years, or over \\
- Smoke $\geq 5$ cpd at the time of enrolment into the trial
\end{tabular}


Bell 2017 (Continued)

- Have been smoking for at least 12 months

- Willing to attempt to quit tobacco smoking after study enrolment

Exclusion criteria:

- Participating in a smoking-cessation programmed

- Pregnant or breastfeeding or planning to be during trial period

- Experienced chest pain, or another cardiovascular event or procedure in the last month

- Being treated with oxygen therapy

Inclusion based on specific population characteristic: People living with HIV

29 participants identified as male, and 1 participant did not identify as male or female; Mean age: 42; Mean cpd: 18

EC use at baseline: $46.7 \%(n=14)$ Never tried; 50\% $(n=15)$ Tried, never used for an extended period; $3.3 \%(n=1)$ Used on a regularly (weekly) basis

Willing to attempt to quit

Interventions

\section{EC: Refillable}

Single-arm study. Print materials to help quit smoking. Provided booklet with instructions on how to use, store and handle EC; copies of device user manuals. Given Innokin Endura T18 ${ }^{\circledR}$ vaporiser kit, Innokin Endura T22 ${ }^{\circledR}$ vaporiser kit, 4 spare coils, 1 wall charger, $10 \times 10$-mL bottles of Nicophar ${ }^{\circledR} 12 \mathrm{mg}$ nicotine e-liquid. Supplies to last 12 weeks

Outcomes Weeks 1, 4, 8, 12, 24; Self-report and semistructured interviews

Cessation: 7 days point prevalence at weeks 4, 8, 12 and 24. Continuous abstinence at weeks 12 and 24 . No biochemical validation

Adverse events

Other outcomes: Acceptability and use of trial products; Number of quit attempts

Study funding

"This work was supported by the HIV Foundation Queensland. The funder will play no role in the analysis and interpretation of results. All trial products were purchased and the suppliers have no involvement in the conduct of the trial or the interpretation or reporting of the results."

Author declarations

"No other authors declare conflicts of interest. Mark Boyd has received research grant funding (paid to the institution) from AbbVie, Gilead and Merck and received honoraria for participation in HIV Advisory Boards and for the preparation and delivery of educational materials from AbbVie, Boehringer-Ingelheim, Bristol Myers Squibb, Gilead, Janssen-Cilag, Merck and ViiV Healthcare."

Notes Additional data provided from authors. New for 2020 update

\section{Risk of bias}

\begin{tabular}{lll}
\hline Bias & Authors' judgement & Support for judgement \\
\hline $\begin{array}{l}\text { Random sequence genera- } \\
\text { tion (selection bias) }\end{array}$ & High risk & Uncontrolled study \\
\hline $\begin{array}{l}\text { Allocation concealment } \\
\text { (selection bias) }\end{array}$ & High risk & Uncontrolled study \\
\hline $\begin{array}{l}\text { Incomplete outcome data } \\
\text { (attrition bias) } \\
\text { All outcomes }\end{array}$ & Low risk & $\begin{array}{l}\text { Quote: "At Week 24, 26 of the 30 participants who enrolled in the study were } \\
\text { followed up." (confirmed by authors) }\end{array}$ \\
\hline
\end{tabular}


Bell 2017 (Continued)

Selective reporting (re- Low risk Study not published at time of data extraction, but study protocol published porting bias)

Bullen 2013

\section{Study characteristics}

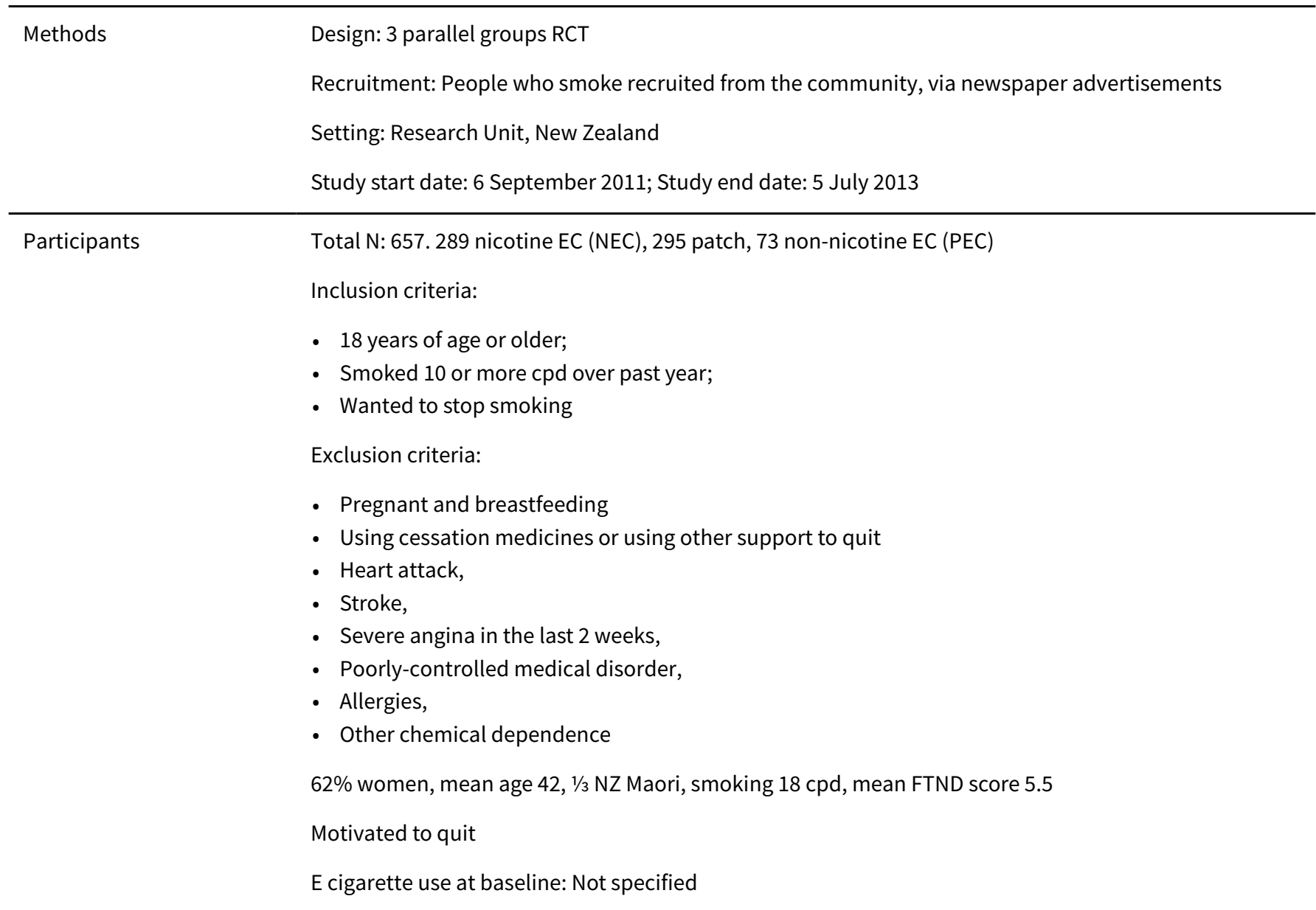

Interventions

\section{EC: Cig-a-like}

Randomized to NEC, PATCH or PEC use for 13 weeks (from 1 week prior to TQD)

- NEC: Elusion brand $16 \mathrm{mg}$ cartridges; sent product via courier

- PATCH: $21 \mathrm{mg} / 24$-hour patch; sent voucher to exchange for NRT at pharmacy (dispensing costs covered)

- PEC: As per EC, but 0 mg cartridges

All participants referred to Quitline and received an invitation to access phone- or text-based support. This was accessed by $<10 \%$

Outcomes

Sustained ( $\leq 5$ cigarettes allowed) validated (exhaled breath $\mathrm{CO}<10 \mathrm{ppm}$ ) abstinence at 6 months

$\geq 50 \%$ self-reported reduction in baseline cigarettes at 6 months

Participants reporting any adverse events

Proportion of AEs that were serious 
Bullen 2013 (Continued)

$$
\text { Proportion of unrelated AES }
$$

Study funding Health Research Council of New Zealand

Author declarations

"We declare that we have received no support from any companies for the submitted work and have no non-financial interests that might be relevant to the submitted work. ML, via his company Health New Zealand, previously did research funded by Ruyan (an e-cigarette manufacturer). CB and HM have done research on Ruyan e-cigarettes funded by Health New Zealand, independently of Ruyan. HM has received honoraria for speaking at research symposia, has received benefits in kind and travel support from, and has provided consultancy to, the manufacturers of smoking cessation drugs. NW has provided consultancy to the manufacturers of smoking cessation drugs, received honoraria for speaking at a research meeting and received benefits in kind and travel support from a manufacturer of smoking cessation drugs. JW has provided consultancy to the manufacturers of smoking cessation medications."

Notes Accessed support: NEC: 115/289; PATCH: 106/295; PEC: 26/73

\section{Risk of bias}

\begin{tabular}{lll}
\hline Bias & Authors' judgement & Support for judgement \\
\hline $\begin{array}{l}\text { Random sequence genera- } \\
\text { tion (selection bias) }\end{array}$ & Low risk & Computerized block randomization \\
\hline $\begin{array}{l}\text { Allocation concealment } \\
\text { (selection bias) }\end{array}$ & Low risk & Computerized via study statistician \\
\hline $\begin{array}{l}\text { Blinding of participants } \\
\text { and personnel (perfor- } \\
\text { mance bias) }\end{array}$ & Low risk & $\begin{array}{l}\text { NEC and PEC were blind to treatment condition in relation to one another. No } \\
\text { bll outcomes } \\
\text { active treatments performance bias judged unlikely }\end{array}$ \\
\hline $\begin{array}{l}\text { Blinding of outcome as- } \\
\text { sessment (detection bias) } \\
\begin{array}{l}\text { All outcomes } \\
\text { Incomplete outcome data }\end{array}\end{array}$ & Low risk & Low risk \\
$\begin{array}{l}\text { (attrition bias) } \\
\text { All outcomes }\end{array}$ & Biochemical validation used \\
\hline $\begin{array}{l}\text { Selective reporting (re- } \\
\text { porting bias) }\end{array}$ & Low risk & $\begin{array}{l}\text { LTFU 22\% (all considered to be smoking). Patch group had a higher LTFU and } \\
\text { withdrawal than EC (loss to follow-up 17\% NEC, 27\% patches, 22\% PEC). How- } \\
\text { ever, minimal difference in per-protocol and ITT analyses }\end{array}$ \\
\hline
\end{tabular}

\section{Caponnetto 2013a}

\section{Study characteristics}

Mesign: 3-arm double-blind randomized controlled trial: EC with 7.2 mg nicotine for 12 weeks; same for
6 weeks followed by $5.2 \mathrm{mg}$ for 6 weeks: EC with no nicotine for 12 weeks
Recruitment: Newspaper advertisements
Setting: Outpatient clinic, Italy
Study start date: April 2010; Study end date:April 2012

\section{Participants}

Total N: 300 
Inclusion criteria:

- Smoked at least $10 \mathrm{cpd}$ for past 5 years;

- Age 18-70

- In good health

- Not currently or intending to quit smoking in the next 30 days

Exclusion criteria:

- Symptomatic cardiovascular or respiratory disease

- Regular psychotropic medicine use

- Current or past history of alcohol abuse

- Use of smokeless tobacco or NRT

- Pregnant or breastfeeding

$36 \%$ women, mean age 44 (SD 12.5), mean cpd 20 (IQR: 15 - 25)

Not currently or intending to quit smoking in the next 30 days

E cigarette use at baseline: Not specified

\section{Interventions $\quad$ EC: Cig-a-like}

EC presented as a healthier alternative to tobacco smoke and could be freely used, ad libitum (up to 4 cartridges a day) for 12 weeks, as a tobacco substitute

EC used: 'Categoria' (model 401) with disposable cartridges

- Grp A: 12 weeks of 7.2 mg capsules ('Original')

- Grp B: 6 weeks $7.2 \mathrm{mg}$ ('Original'), then 6 weeks $5.4 \mathrm{mg}$ ('Categoria')

- Grp C: 12 weeks of $0 \mathrm{mg}$ ('Original')

Baseline visit and up to 7 follow-up visits to receive more cartridges, hand-in diaries, measure $\mathrm{CO}$ and vital signs

Outcomes

Abstinence at 12 months (complete self-reported abstinence from tobacco smoking since previous visit at 6 months, confirmed with $\mathrm{CO}<7 \mathrm{ppm}$ at 12 months)

$\geq 50 \%$ reduction in baseline cigarettes at 12 months

Recorded AEs thought to be related to tobacco smoking and EC at baseline and at each study visit (7 follow-up visits over 12 weeks, plus at 24 and 52 weeks)

Study funding

"This research was supported by a grant-in-aid from Lega Italiana AntiFumo. The study sponsor had no involvement in the study design, collection, analysis, and interpretation of data, the writing of the manuscript or the decision to submit the manuscript for publication. RP and PC are currently funded by the University of Catania, Italy. The e-cigarette supplier had no involvement in the study design, collection, analysis, and interpretation of data, the writing of the manuscript or the decision to submit the manuscript for publication."

Author declarations

"RP has received lecture fees and research funding from Pfizer and GlaxoSmithKline, manufacturers of stop smoking medications. He has served as a consultant for Pfizer and Arbi Group Srl, the distributor of the CategoriaTM e-Cigarette. The other authors have no relevant conflict of interest to declare in relation to this work."

Notes Additional data provided from authors

\section{Risk of bias}


Caponnetto 2013a (Continued)

Random sequence genera- Low risk Computer-generated, block size 15 (5:5:5 ratio) tion (selection bias)

\begin{tabular}{|c|c|c|}
\hline $\begin{array}{l}\text { Allocation concealment } \\
\text { (selection bias) }\end{array}$ & Low risk & $\begin{array}{l}\text { Randomization carried out by pharmacy, who did not have direct contact with } \\
\text { the participants }\end{array}$ \\
\hline $\begin{array}{l}\text { Blinding of participants } \\
\text { and personnel (perfor- } \\
\text { mance bias) } \\
\text { All outcomes }\end{array}$ & Low risk & $\begin{array}{l}\text { Double-blind. } \\
\text { Quote: "Blinding was ensured by the identical external appearance of the car- } \\
\text { tridges. The hospital pharmacy was in charge of randomization and packaging } \\
\text { of the cigarettes" }\end{array}$ \\
\hline
\end{tabular}

\begin{tabular}{lll}
\hline $\begin{array}{l}\text { Blinding of outcome as- } \\
\text { sessment (detection bias) } \\
\text { All outcomes }\end{array}$ & Low risk & Biochemical validation used \\
\hline $\begin{array}{l}\text { Incomplete outcome data } \\
\text { (attrition bias) } \\
\text { All outcomes }\end{array}$ & Low risk & $\begin{array}{l}211(70.3 \%) \text { and } 183(61 \%) \text { attended 6- and 12-month follow-up (at 12 m, 35\% } \\
\text { lost in 7.2 group; 37\% lost in 5.4 group; 45\% lost in no-nicotine group) }\end{array}$ \\
\hline $\begin{array}{l}\text { Selective reporting (re- } \\
\text { porting bias) }\end{array}$ & Unclear risk & $\begin{array}{l}\text { Unclear if original intention was to combine groups A+B or not. In sample size } \\
\text { calculation they compared A+B with C, but results are not always reported in } \\
\text { this way }\end{array}$ \\
\hline
\end{tabular}

\section{Study characteristics}

\begin{tabular}{|c|c|}
\hline Methods & $\begin{array}{l}\text { Design: Prospective cohort } \\
\text { Recruitment and setting: Inpatients at a psychiatric institution in Italy } \\
\text { Study start date/end date: Not specified }\end{array}$ \\
\hline Participants & $\begin{array}{l}\text { Total N: } 14 \\
\text { Inclusion criteria: } \\
\text { - Smoked } \geq 20 \text { cpd for at least the past } 10 \text { years } \\
\text { - Diagnosis of schizophrenia } \\
\text { Exclusion criteria: } \\
\text { - Alcohol and illicit drug use } \\
\text { - Recent myocardial infarction } \\
\text { - Angina pectoris } \\
\text { - High blood pressure (BP > } 140 \text { mmHg systolic or } 90 \text { mmHg diastolic, or both) } \\
\text { - Diabetes mellitus } \\
\text { - Severe allergies } \\
\text { - Poorly-controlled asthma or other airway diseases } \\
\text { - Inclusion based on specific population characteristic: Diagnosis of schizophrenia } \\
57 \% \text { women, mean age } 44.6 \text { (SD } 12.5 \text { ), mean pack years smoked } 28.8 \text { (SD 12.9) } \\
\text { Motivated to quit: Not specified } \\
\text { E cigarette use at baseline: Not specified }\end{array}$ \\
\hline
\end{tabular}


Caponnetto 2013b (Continued)

Interventions

\section{EC: Cig-a-like}

Seen at baseline, given EC ('Categoria' brand) with an initial 4-week supply of 7.4 mg nicotine cartridges. Instructed to use ad libitum up to 4 cartridges a day. EC cartridges supplied at months 1,2 , and 3

No instruction on cessation or reduction was provided.

\begin{tabular}{ll}
\hline Study funding & $\begin{array}{l}\text { "We wish to thank Arbi Group Srl (Milano, Italy) for the free supplies of "Categoria" e-cigarette kits and } \\
\text { nicotine cartridges as well as their support. We would also like to thank LIAF (Lega Italiana AntiFumo) } \\
\text { for the collaboration." }\end{array}$ \\
\hline Author declarations & $\begin{array}{l}\text { "Pasquale Caponnetto, Roberta Auditore, Cristina Russo and Giorgio Carlo Cappello declare no con- } \\
\text { flict of interest. Riccardo Polosa has received lecture fees and research funding from Pfizer and Glax- } \\
\text { oSmithKline, manufacturers of stop smoking medications. He has served as a consultant for Pfizer and } \\
\text { Arbi Group Srl (Milano, Italy), the distributor of the CategoriaTM e-cigarette." }\end{array}$
\end{tabular}

Notes

\title{
Risk of bias
}

\begin{tabular}{lll}
\hline Bias & Authors' judgement & Support for judgement \\
\hline $\begin{array}{l}\text { Random sequence genera- } \\
\text { tion (selection bias) }\end{array}$ & High risk & Prospective cohort; no randomization \\
\hline $\begin{array}{l}\text { Allocation concealment } \\
\text { (selection bias) }\end{array}$ & High risk & Not randomized \\
\hline $\begin{array}{l}\text { Incomplete outcome data } \\
\begin{array}{l}\text { (attrition bias) } \\
\text { All outcomes }\end{array}\end{array}$ & Low risk & 0/14 lost to follow-up \\
\hline $\begin{array}{l}\text { Selective reporting (re- } \\
\text { porting bias) }\end{array}$ & Unclear risk & Unable to determine prespecified outcomes \\
\hline
\end{tabular}

Carpenter 2017

\section{Study characteristics}

Methods

\author{
Design: Randomized parallel-assignment open-label trial \\ Recruitment: Recruitment from local urban community in southeastern USA, using various media out- \\ lets \\ Setting: Community, southeastern USA \\ Study start date: November 2014; Study end date: May 2016
}


Carpenter 2017 (Continued)

Participants
Total N: 68

$\mathrm{N}$ per arm: Control group: 22; ENDS group: 46 (split into 2 non-randomized groups: BluCig $16 \mathrm{mg}$ : 25; BluCig $24 \mathrm{mg}: 21$ )

Inclusion criteria:

- Age 18+

- Current smoker of $\geq 5 \mathrm{cpd}$ for $\geq 1$ year

- No recent history of cardiovascular distress, COPD, cancer (any non-dermatologic), or uncontrolled diabetes mellitus

- Neither pregnant nor breastfeeding (verified)

- Absence of any major current psychiatric impairment, including current alcohol/drug abuse/dependence

- Current, active use of email

- At least some concern for health effects of smoking (> none at all on a Likert scale)

- Not used any ENDS product in the past 6 months

- Never purchased an ENDS product

Exclusion criteria:

- Use of non-cigarette tobacco products (e.g. cigarillos) in the last 30 days

- Current use of any smoking cessation medications

- Current enrolment in a smoking cessation treatment study

Women: 59.7\%; Mean age: 42.2; Mean cpd: 15.3; Heaviness of smoking (0 - 6): 2.9

EC use: Control: 9\%; ENDS 16 mg group: 4\%; ENDS 24mg group: 33\%

Motivation to quit smoking in next month (0 - 10): Control: 4.0; ENDS 16 mg: 5.0; ENDS 24 mg: 4.4

\section{EC: Cig-a-like}

Intervention: At study start, choice of tobacco or menthol flavor Blu Starter Pack EC, with $16 \mathrm{mg} / \mathrm{mL}$ nicotine. Midway through study, the manufacturer of Blu altered the product and discontinued availability of the device, replaced with BluPlusp, with $24 \mathrm{mg} / \mathrm{mL}$ nicotine. 3-week sampling period, given up to 7 cartridges at each of 3 weekly visits. Instructions on usage "kept minimal to preserve naturalistic intent." The study team suggested that ENDS could be used "as you wish, to cut down or quit smoking, help manage smoking restrictions, or both."

Control: own brand of cigarettes

\begin{tabular}{ll}
\hline Study funding & "Support was provided by NIH R21 DA037407 (to M.J. Carpenter), P01 CA200512 (to K.M. Cummings, \\
& M.J. Carpenter, and M.L. Goniewicz), UL1 TR001450, and P30 CA138313. M.L. Goniewicz's laboratory is \\
& supported via P30 CA016056. B.W. Heckman is supported via K12 DA031794 and K23 DA041616. T.L. Wa- \\
& gener's effort is partially supported by the Oklahoma Tobacco Research Center, which is funded by the \\
& Oklahoma Tobacco Settlement Endowment Trust."
\end{tabular}

Author declarations
"M.L. Goniewicz is a consultant/advisory board member for Johnson \& Johnson. K.M. Cummings reports receiving a commercial research grant from and is a consultant/advisory board member for Pfizer Inc., and has provided expert witness testimony for various plaintiffs in lawsuits involving cigarette manufacturers. No potential conflicts of interest were disclosed by the other authors." 
Carpenter 2017 (Continued) Notes
New for 2020 update. Listed as ongoing study NCT02357173 in 2016 review update. Additional data provided from authors

In all, 25 participants (54\%) received the Blu Starter Pack ( $16 \mathrm{mg})$, and 21 participants $(46 \%)$ received BluPlusp (24 mg); no switches were made within participants. Note: this is not included in our analysis of higher v lower as assignment to nicotine dose was not done at random; $24 \mathrm{mg}$ and $16 \mathrm{mg}$ merged in our main analysis

\section{Risk of bias}

\begin{tabular}{lll}
\hline Bias & Authors' judgement & Support for judgement \\
\hline $\begin{array}{l}\text { Random sequence genera- } \\
\text { tion (selection bias) }\end{array}$ & Unclear risk & $\begin{array}{l}\text { Quote: "Randomization to group was stratified by motivation to quit in the } \\
\text { next 30 days (0-6 vs. 7-10 on a VAS scale) but proportioned 2:1 (ENDS:control) } \\
\text { to increase precision estimates for e-cigarette uptake and usage." }\end{array}$ \\
\hline $\begin{array}{l}\text { Allocation concealment } \\
\text { (selection bias) }\end{array}$ & Unclear risk & Not specified \\
\hline $\begin{array}{l}\text { Blinding of participants } \\
\text { and personnel (perfor- } \\
\text { mance bias) } \\
\text { All outcomes }\end{array}$ & High risk & Not blinded and includes non-active control \\
\hline
\end{tabular}

\section{Blinding of outcome as- High risk} sessment (detection bias) All outcomes

Incomplete outcome data Low risk (attrition bias)

All outcomes
CO biochemically verified but abstinence not used as outcome in this review, so rated based on adverse event reporting. Self-report, no blinding of participants.

\author{
Retention rate: \\ Week 4: Control:19/22 (86\%); ENDS 16 mg: 23/25 (92\%); ENDS 24 mg: 20/21 \\ (95\%) \\ Week 16: Control: 16/22 (73\%); ENDS 16 mg: 19/25 (76\%); ENDS 24 mg: 15/21 \\ (71\%)
}

Selective reporting (re- Unclear risk Not specified
porting bias)

\begin{tabular}{ll}
\hline Other bias & $\begin{array}{l}\text { Midway through the study, the manufacturer of Blu altered the product and } \\
\text { discontinued availability of the device, replaced with BluPlusp, with } 24 \mathrm{mg} /\end{array}$ \\
$\mathrm{mL}$ nicotine, again offered in both tobacco and menthol flavorings, and with \\
improved battery duration (4-watt battery for both devices). In all, 25 partici- \\
pants $(54 \%)$ received the Blu Starter Pack (16 mg), and 21 participants (46\%) \\
received BluPlusp ( $24 \mathrm{mg})$; no switches were made within participants. The \\
change in product (IRB approved) allowed us the unexpected opportunity to \\
assess what impact, if any, the change in product design had on study out- \\
comes. Note that the manufacturer, style of device, and packaging did not \\
change, nor did our messaging to participants. The only difference was the \\
strength of product. Thus, trial outcomes are reported across 3 groups: control \\
versus 16 mg versus 24 mg ENDS. We have not rated this as high risk of bias as \\
our analyses do not compare on nicotine strength and both nicotine arms are \\
combined in our main analysis
\end{tabular}

\section{Study characteristics}


Czoli 2019 (Continued)

Methods
Design: Nonblinded within-participants cross-over

Recruitment: advertisements placed in newspapers, online, and in local vape shops, and received CAD 295 for participating in the study

Setting: Kitchener-Waterloo and Toronto, Ontario, Canada

Study start date: September 2015. Study end date: NR

Participants

29.2\% female; mean age 35.9 (SD 11.7); mean cpd NR; dual EC users at baseline; not motivated to quit Inclusion criteria:

- $>18$

- Dual user s of tobacco cigarettes and e-cigarettes. Dual users were identified as current daily tobacco cigarette smokers (had smoked $\geq 100$ cigarettes in their lifetime, and smoked $\geq 5$ cigarettes/day) and current daily e-cigarette users (had used an e-cigarette at least once a day for each of the past 7 days)

Exclusion criteria:

- Serious intentions to quit smoking in the next 6 months

- use of other tobacco products in the past 7 days

- use of nicotine replacement therapy in the past 7 days

- use of any smoking cessation medications in the past 7 days

- participation in individual or group counseling programs for smoking cessation in the past 7 days

- experience of serious cardiac health issues

- experience of a heart attack or stroke within the last 3 months

- experience of cancer within the last year

- experience of asthma, chronic obstructive pulmonary disease, a seizure disorder, or any life-threatening medical conditions with a prognosis of less than a year

- a history of psychosis, schizophrenia, bipolar disorder, or suicidal thoughts

\section{EC: own choice (mainly tank)}

3 consecutive 7 -day periods in which the use of tobacco cigarettes and e-cigarettes was experimentally manipulated

4 study conditions: Dual use (e-cigarette and tobacco cigarette); Tobacco cigarette; E-cigarette; No product use

Virtually all dual users reported using tank systems (92\%) and e-cigarettes with nicotine (94\%)

To control for order effects, participants were randomly assigned to 1 of 2 condition orders, A or B

Following the baseline condition of dual use:

Group A participants switched to E-cigarette use, then to Tobacco cigarette use, and finally to No product use

Group B participants switched to Tobacco cigarette use, then to E-cigarette use, and finally to No product use

Outcomes

Baseline (visit 1) and after each of the 7-day periods (visit 2 (week 1), visit 3 (week 2), visit 4 (week 3))

Carbon monoxide

Urinary concentration of cotinine 
Czoli 2019 (Continued)

Urinary concentrations of 1-hydroxypyrene (1-HOP) and 4-(methylnitrosamino)-1-(3-pyridyl)-1-butanol (NNAL)

Study funding

This research was supported by an Ontario Ministry of Health and LongTerm Care Health System Research Fund grant (\#06697 awarded to DH). Additional support was provided by the Canadian Institutes of Health Research (CIHR), the Vanier Canada Graduate Scholarship (CDC), a CIHR and Public Health Agency of Canada, Applied Public Health Chair (DH), and an Ontario Institute for Cancer Research Investigator Award (GTF)
MLG reports grants from and served as an advisory board member to pharmaceutical companies that manufacture smoking cessation drugs. DH has provided paid expert testimony in tobacco litigation on behalf of governments and class-action plaintiffs on issues related to tobacco product science and regulation. The other authors have no competing interests to declare

Notes New for 2021 update

\section{Risk of bias}

\begin{tabular}{lll}
\hline Bias & Authors' judgement & Support for judgement \\
\hline $\begin{array}{l}\text { Random sequence genera- } \\
\text { tion (selection bias) }\end{array}$ & Unclear risk & No details of randomization method given \\
\hline $\begin{array}{l}\text { Allocation concealment } \\
\text { (selection bias) }\end{array}$ & High risk & No blinding \\
\hline $\begin{array}{l}\text { Blinding of participants } \\
\text { and personnel (perfor- } \\
\text { mance bias) }\end{array}$ & High risk & No blinding \\
All outcomes & \\
\hline $\begin{array}{l}\text { Blinding of outcome as- } \\
\text { sessment (detection bias) } \\
\text { All outcomes }\end{array}$ & Unclear risk & Not reported \\
\hline $\begin{array}{l}\text { Incomplete outcome data } \\
\text { (attrition bias) } \\
\text { All outcomes }\end{array}$ & Low risk & All followed up \\
\hline $\begin{array}{l}\text { Selective reporting (re- } \\
\text { porting bias) }\end{array}$ & Low risk & No evidence of selective reporting \\
\hline
\end{tabular}

Dawkins 2020

\section{Study characteristics}

\begin{tabular}{ll}
\hline Methods & Design: Prospective cohort 4-center pragmatic cluster feasibility trial \\
& Recruitment: At homeless centers \\
& Setting: 4 homeless centers in the UK \\
Study start date: 1 October $2018 ;$ Study end date: 31 March 2020 \\
\hline Participants \\
Total N: 80 \\
N per arm: EC 48; UC 32
\end{tabular}


Dawkins 2020 (Continued)

Inclusion criteria:

- Adults who smoke (18 and over) accessing homeless support services on a regular basis and also known to staff

- Self-reported daily smokers only with smoking status also confirmed by support staff

- Smoking status was also biochemically verified by exhaled $\mathrm{CO}$ breath

Exclusion criteria:

- Non-smokers, or those reporting using another smoking cessation aid at the current time

- Anyone below the age 18 years, reporting pregnancy, or unable to consent, e.g. currently intoxicated or unable to speak English

- All those not well known to centre staff were ineligible

Inclusion based on specific population characteristic: people accessing homeless centers

35\% women; mean age 42.7; mean cpd 20; mean FTND: FTCD 5.51

Motivated to quit: "varied considerably; large majority expressed a desire to quit smoking in the near future"

E-cigarette use at baseline: Not specified

\begin{abstract}
Interventions
EC: Refillable

Usual care: Written information on quitting smoking (adapted from NHS Choices); signposting to the local stop-smoking service (SSS) by center staff

Intervention: as usual care, plus refillable EC provided once with e-liquid provided $1 \mathrm{x}$ wk for 4 weeks, Aspire PockeX (tank style), choice of 3 flavors (fruit, menthol, tobacco) and 2 nicotine strengths (12 mg/ $\mathrm{mL}$ or $18 \mathrm{mg} / \mathrm{mL}$ ). Written info for EC use and support from center staff, who met once a week to provide e-liquid and troubleshoot EC use
\end{abstract}

Weeks: $4,12,24 ;$ Clinic visits and self-report
Cessation: CO-validated sustained at 24 weeks
Adverse events and biomarkers: Self-reported negative effects in EC arm only - each participant asked
to rate on scale so cannot meta-analyse; exhaled CO; unintended consequences
Other outcomes measured:
Qualitative process evaluation; costs; self-reported positive and negative affects; recruitment rates; re-
tention; EC/other tobacco/nicotine product use at study end; HRQL; healthcare service utilization;
other drug use/dependence; unintended consequences

Study funding

This study is funded by the National Institute for Health Research Public Health (project reference: $17 / 44 / 29)$

Author declarations

$\mathrm{SC}, \mathrm{AF}, \mathrm{JL}, \mathrm{CB}, \mathrm{AT}, \mathrm{DR}, \mathrm{IU}, \mathrm{LB}, \mathrm{SP}$ have no competing interests. $\mathrm{PH}$ has received research grant from and provided consultancy to Pfizer. LD has provided consultancy for the pharmaceutical industry relating to the development of smoking cessation products

Notes New for 2021 update. Authors provided information prior to peer review

\title{
Risk of bias
}

\begin{tabular}{lll}
\hline Bias & Authors' judgement & Support for judgement \\
\hline $\begin{array}{l}\text { Random sequence genera- } \\
\text { tion (selection bias) }\end{array}$ & High risk & Intention was to randomize but were unable to due to practical constraints
\end{tabular}


Quote: "Thus the actual allocation of centres to each arm was a pragmatic decision based on centre readiness and staff/researcher availability though we balance potential confounders and differences in environment by ensuring each cluster (EC and UC) contained one day centre and one residential unit."

\begin{tabular}{|c|c|c|}
\hline $\begin{array}{l}\text { Allocation concealment } \\
\text { (selection bias) }\end{array}$ & Unclear risk & $\begin{array}{l}\text { Quote: "Participants joined after cluster randomisation... Allocation was con- } \\
\text { cealed to participants until after the baseline assessment." } \\
\text { Comment: But unclear if allocation was concealed for those recruiting, and al- } \\
\text { location would have been known to new participants }\end{array}$ \\
\hline $\begin{array}{l}\text { Blinding of participants } \\
\text { and personnel (perfor- } \\
\text { mance bias) } \\
\text { All outcomes }\end{array}$ & High risk & $\begin{array}{l}\text { Not blinded and different levels of support between arms, so performance bias } \\
\text { cannot be ruled out }\end{array}$ \\
\hline $\begin{array}{l}\text { Blinding of outcome as- } \\
\text { sessment (detection bias) } \\
\text { All outcomes }\end{array}$ & Low risk & Cessation (primary outcome) biochemically-validated \\
\hline $\begin{array}{l}\text { Incomplete outcome data } \\
\text { (attrition bias) } \\
\text { All outcomes }\end{array}$ & High risk & $\begin{array}{l}13 / 48(27.1 \%) \text { lost to follow-up in the intervention arm and } 20 / 32(62.5 \%) \text { lost } \\
\text { to follow-up in the control arm at } 24 \text { weeks }\end{array}$ \\
\hline $\begin{array}{l}\text { Selective reporting (re- } \\
\text { porting bias) }\end{array}$ & Low risk & All anticipated outcomes reported \\
\hline
\end{tabular}

\section{Eisenberg 2020}

\section{Study characteristics}

Design: 3-arm RCT
Recruitment: Community
Setting: Canada
Study start date: November 2016. Study end date: September 2019.
Total N: 376; Nicotine e-cigarettes = 128; Non-nicotine e-cigarettes = 127; Counselling (control) = 121
47\% female; mean age 52.66; mean cpd 21; mean FTND 6 (SD 2).
Motivated to quit - Yes
Inclusion criteria:
- Active smoker, 10 or more cigarettes per day, on average, for the past year
- Age of 18 years or older
- Motivated to quit according to the Motivation To Stop Scale (MTSS) (level 5 or higher)
- Able to understand and to provide informed consent in English or French
- Likely to be available for follow-up (1 year)
Exclusion criteria:
- Medical condition with a prognosis < 1 year
- Current or recent cancer (less than 1 year in remission)
- Pregnant or lactating women


- Current or recent use (in the past 30 days) of any pharmacotherapy or behavioral therapy for smoking cessation (e.g. nicotine replacement

- Therapies, bupropion, varenicline, or counseling)

- Any e-cigarette use (nicotine or non-nicotine) in the past 60 days, or ever use of any e-cigarette for more than 7 days consecutively

- History of psychosis, schizophrenia, or bipolar disorder

- Less than 1 month following a myocardial infarction, life-threatening arrhythmia, severe or worsening angina pectoris, or cerebral vascular accident

- Use of any illegal drugs in the past year (excluding marijuana)

- Planned use of tobacco products other than conventional cigarettes (e.g. cigarillos, cigars, snuff, shisha, etc.) or marijuana during the study period

Interventions

\section{EC: Cig-a-like}

\section{Nicotine e-cigarettes plus counseling:}

12 weeks of e-cigarettes. Rechargeable base with prefilled, disposable, tobacco-flavored liquid cartridges (15 or $0 \mathrm{mg}$ nicotine/ $\mathrm{mL}$ ), which were produced specifically for use in clinical studies (purchased from NJOY Inc, Scottsdale, Arizona). 21 cartridges at baseline with additional cartridges supplied as needed. Nicotine and nonnicotine e-cigarettes were identical in appearance. Instructed to be used as desired. No schedule for e-cigarette tapering, but participants were aware that they would return their e-cigarettes after 12 weeks

Participants received individual smoking cessation and relapse prevention counseling (minimum 30 minutes at baseline, 10 minutes during telephone follow-ups, and 15 - 20 minutes at clinic visits). Individualized quit plans

\section{Non-nicotine e-cigarettes plus counseling:}

As above with $0 \mathrm{mg}$ nicotine $/ \mathrm{mL}$ in liquid cartridge

\section{Counseling (control):}

Participants received individual smoking cessation and relapse prevention counseling (minimum 30 minutes at baseline, 10 minutes during telephone follow-ups, and 15 - 20 minutes at clinic visits). Individualized quit plans

Outcomes

Follow-up was conducted by telephone at weeks $1,2,8$, and 18, and at clinic visits at weeks 4, 12, 24, and 52

Self-reported smoking (7-day recall), adherence, and adverse events (AEs) were assessed during follow-up contacts

Biochemically-validated 7-day point prevalence smoking abstinence at 4, 12 and 24 weeks, defined as self-reported abstinence in the past 7 days with exhaled carbon monoxide $<11 \mathrm{ppm}$

At baseline: cpd; FTND; Glover-Nilsson Smoking Behavioral Questionnaire (to assess behavioral dependence on smoking); and Beck Depression Inventory II (BDI-II; to assess depressive symptoms)

Study funding

This trial was funded by the Canadian Institutes of Health Research (CIHR; funding reference No. 133727 and 155969). Both nicotine e-cigarettes and nonnicotine e-cigarettes were purchased from NJOY Inc (Scottsdale, Arizona)

Author declarations

Dr Eisenberg reported receiving educational grants from Pfizer Inc for providing continuing medical education in cardiology. Dr Wilderman reported receiving financial compensation from Pfizer Inc for his involvement in a smoking cessation study using varenicline. Dr Filion reported receiving salary support from the Fonds de Recherche du Quebec, a William Dawson Scholar award from McGill University, and personal fees from Institut National D'excellence en Santé et Services Sociaux. No other disclosures were reported 
Eisenberg 2020 (Continued)

Notes

New cessation and adverse event data for 2021 update. Previously listed as NCT02417467 (included with SAE data only)

\section{Risk of bias}

\begin{tabular}{|c|c|c|}
\hline Bias & Authors' judgement & Support for judgement \\
\hline $\begin{array}{l}\text { Random sequence genera- } \\
\text { tion (selection bias) }\end{array}$ & Low risk & $\begin{array}{l}\text { Eligible participants were randomized via an online central randomization sys- } \\
\text { tem. The system used a computer-generated randomization list containing } \\
\text { permuted blocks of } 6 \text { and } 9 \text {, stratified by center }\end{array}$ \\
\hline $\begin{array}{l}\text { Allocation concealment } \\
\text { (selection bias) }\end{array}$ & Low risk & $\begin{array}{l}\text { Participants, investigators, and study personnel were blinded to nicotine con- } \\
\text { tent in the e-cigarette groups }\end{array}$ \\
\hline $\begin{array}{l}\text { Blinding of participants } \\
\text { and personnel (perfor- } \\
\text { mance bias) } \\
\text { All outcomes }\end{array}$ & Low risk & $\begin{array}{l}\text { Participants, investigators, and study personnel were blinded to nicotine con- } \\
\text { tent in the e-cigarette groups }\end{array}$ \\
\hline $\begin{array}{l}\text { Blinding of outcome as- } \\
\text { sessment (detection bias) } \\
\text { All outcomes }\end{array}$ & Low risk & $\begin{array}{l}\text { Participants, investigators, and study personnel were blinded to nicotine con- } \\
\text { tent in the e-cigarette groups }\end{array}$ \\
\hline $\begin{array}{l}\text { Incomplete outcome data } \\
\text { (attrition bias) } \\
\text { All outcomes }\end{array}$ & Low risk & Low numbers lost to follow-up, treated as ITT \\
\hline $\begin{array}{l}\text { Selective reporting (re- } \\
\text { porting bias) }\end{array}$ & Low risk & $\begin{array}{l}\text { Due to a prolonged and unforeseen delay in e-cigarette manufacturing, en- } \\
\text { rolment was paused on } 27 \text { September } 2019 \text {, and then terminated on } 14 \text { No- } \\
\text { vember } 2019 \text {. Given reduced power, the timing of the primary endpoint was } \\
\text { changed from } 52 \text { weeks to } 12 \text { weeks on } 04 \text { December } 2019 \text {. No } 12 \text {-month fol- } \\
\text { low-up but this was for manufacturing reasons and was reported }\end{array}$ \\
\hline
\end{tabular}

\section{Ely 2013}

\section{Study characteristics}

\begin{tabular}{ll} 
Methods & Design: Prospective cohort \\
Recruitment: Letter sent to family practice patients who currently smoked \\
Setting: Single family practice, Colorado USA \\
Study start date: 14 April 2013; Study end date: Not specified \\
\hline Letters sent to 640 patients, 48 chose to participate and 44 completed the program, 4 were lost to fol- \\
low-up \\
Inclusion criteria: \\
- Want to quit or switch from tobacco cigarettes to ECs \\
Exclusion criteria: \\
- None reported \\
Of the 44 participants, $66 \%$ women, all non-Hispanic/white, aged 20 - 75 (30\% were age 51 - 60), 57\% \\
had a high school education or less
\end{tabular}


Ely 2013 (Continued)

Motivated to quit: Want to quit or switch from tobacco cigarettes to ECS

E-cigarette use at baseline: Not specified

EC: Cig-a-like
The 6-month smoking cessation program was based on The '5 A's' model and transtheoretical model.
Options for treatment were discussed with each participant at the start of the program. All used an EC,
with 16 using bupropion and 2 using varenicline as well
Participants were provided with written information on "blu cig" and "smoke tip" ECs, about cost,
availability, nicotine dosage options

Outcomes Phone follow-ups at 2 weeks, 1 month, 3 months, and 6 months

At completion of program (using ITT)

Abstinence from smoking and EC use

Abstinence from smoking but not EC use

$\geq 50 \%$ reduction of baseline cigarette consumption (still using ECs)

\begin{tabular}{ll}
\hline Study funding & Not specified \\
\hline Author declarations & Not specified \\
\hline Notes & \\
\hline
\end{tabular}

\section{Risk of bias}

\begin{tabular}{lll}
\hline Bias & Authors' judgement & Support for judgement \\
\hline $\begin{array}{l}\text { Random sequence genera- } \\
\text { tion (selection bias) }\end{array}$ & High risk & Prospective cohort \\
\hline $\begin{array}{l}\text { Allocation concealment } \\
\text { (selection bias) }\end{array}$ & High risk & Not randomized \\
\hline $\begin{array}{l}\text { Incomplete outcome data } \\
\text { (attrition bias) } \\
\text { All outcomes }\end{array}$ & Low risk & $4 / 48$ lost to follow-up \\
\hline
\end{tabular}

Selective reporting (re- Unclear risk Unable to determine prespecified outcomes
porting bias)

$\begin{array}{ll}\text { Other bias } & \text { Unclear risk } \\ & \text { No definition of abstinence provided } \\ & \text { Not clear if 'completed program' was at } 6 \text { months. }\end{array}$

Felicione 2019

\section{Study characteristics}

\begin{tabular}{ll}
\hline Methods & Design: Double-blind RCT \\
& Recruitment: People who smoke were recruited from an outpatient opioid-maintenance clinic in West \\
& Virginia, USA
\end{tabular}


Felicione 2019 (Continued)

Setting: Outpatient opioid-maintenance clinic in West Virginia, USA

Study start date/Study end date: Not reported

\begin{tabular}{|c|c|}
\hline Participants & $\begin{array}{l}\text { Total N: 25; N per arm: Placebo (non-nicotine): } 11 \text {; Active ( } 18 \mathrm{mg} / \mathrm{ml} \text { nicotine): } 14 \\
\text { Inclusion criteria: } \\
\text { - } \geq 18 \text { years of age } \\
\text { - Report smoking } \geq 10 \mathrm{cpd} \text { for } \geq \text { one year } \\
\text { - Report a current interest in quitting smoking } \\
\text { Exclusion criteria: } \\
\text { - Reported regular use of any nicotine/tobacco product other than cigarettes, including EC, or were } \\
\text { already engaged in attempts to quit smoking } \\
\text { Inclusion based on specific population characteristic: People who smoke who were currently receiving } \\
\text { a buprenorphine/naloxone combination in sublingual form, and had maintained sobriety from opioids } \\
\text { and all other illicit substances for at least } 90 \text { consecutive days as verified via urinalysis } \\
73.0 \% \text { women; mean age } 32.5 \text {; mean cpd } 22 ; \text { mean FTND } 5.8 \\
\text { Motivated to quit: Quit ladder Score (range } 1 \text { - } 10): 5.6 \text { average }\end{array}$ \\
\hline Interventions & $\begin{array}{l}\text { EC: Refillable } \\
\text { Compared nicotine ( } 18 \mathrm{mg} / \mathrm{ml}) \text { to non-nicotine EC. } \\
\text { Second-generation EC consisted of the eGo-T battery ( } 900 \mathrm{mAh}, 3.3 \mathrm{~V} \text { constant output) (Joyetech; } \\
\text { Irvine, CA) and the Kanger mini Protank-II, } 1.5 \mathrm{ml} \text { Pyrex glass tank with a drip tip and atomizer head } \\
\text { coils (KangerTech; China), choice between tobacco }(\mathrm{n}=15) \text { and menthol }(\mathrm{n}=10) \text { flavored liquid ( } 2 \text {-week } \\
\text { supply). Participants were then trained in EC device operation, including assembly, liquid filling, man- } \\
\text { ual battery operation, and cleaning/storage. Practised puffing on EC in the presence of a team mem- } \\
\text { ber, and asked questions if needed. Participants instructed to use their ECIG ad libitum every day for } 2 \\
\text { weeks }\end{array}$ \\
\hline
\end{tabular}

Outcomes Baseline (day 1), 14 days, 28 days for clinic measures. Data also collected via text-messages over $2-$ week intervention period

Withdrawal/side effects: Every evening during the 2-week intervention period, participants rated a variety of effects possibly experienced as a result of nicotine/tobacco withdrawal and/or use of the ECIG: nausea, dizziness, throat irritation/soreness, cough, dry mouth, headache, shortness of breath, irritability/frustration/anger, craving/urge to smoke, and other. Each item was rated on a continuous scale that ranged from 0 (not at all) to 100 (extremely)

Expired air $\mathrm{CO}$

Other outcomes: Self-reported cigarette and EC use; readiness to quit at day 1,14 and 28

\begin{tabular}{ll}
\hline Study funding & Not reported \\
\hline Author declarations & Not reported \\
\hline Notes & New for 2020 update
\end{tabular}

\section{Risk of bias}


Felicione 2019 (Continued)

Random sequence genera- Unclear risk tion (selection bias)

Quote: "Using a mixed factorial, simple randomization, double-blind study design, participants were assigned to one of two ECIG conditions..." (No further details given)

\begin{tabular}{lll}
\hline $\begin{array}{l}\text { Allocation concealment } \\
\text { (selection bias) }\end{array}$ & Unclear risk $\quad$ No details on allocation given.
\end{tabular}

Blinding of participants

Unclear risk

Quote: "double-blind study design”, no further detail given and personnel (perfor-

mance bias)

All outcomes

Blinding of outcome as-
sessment (detection bias) $\quad$ Qunclear risk: "double-blind study design", no further details given.

All outcomes

Incomplete outcome data Low risk

All outcomes (attrition bias)

Quote: “...80.6\% completed the two-week intervention ( $\mathrm{n}=14$ active; $\mathrm{n}=11$ placebo), and $70.9 \%$ also completed the follow-up session ( $n=13$ active; $n=9$ placebo)."

Active follow-up completion rate: $13 / 14=93 \%$; Placebo follow-up completion rate: $9 / 11=82 \%$

N.B. 6 participants were disqualified post-randomization:

Quote: "Of those individuals who were screened for the study, 93.9\% were enrolled ( $\mathrm{n}=18$ active; $\mathrm{n}=13$ placebo); two individuals who were ineligible provided an expired air CO level $<10 \mathrm{ppm}$. Six of the enrolled participants ( $n=4$ active and $n=2$ placebo; $n=5$ tobacco flavor and $n=1$ menthol flavor) were disqualified for responding to 7 or fewer days of text messages."

Selective reporting (re- Unclear risk porting bias)
All measures listed were reported: Self-reported cigarette use, text message-based cigarette use, e-cig use, expired air CO, readiness to quit ladder, withdrawal/side effect;

No study protocol or clinical trial record available to confirm all intended outcome measures were reported

\section{Study characteristics}

Methods Design: Prospective, randomized controlled trial with a parallel, nonrandomized preference cohort

Recruitment: Participants were recruited from local advertisements, smoking cessation databases, and visits to local businesses, as well as via the Scottish Primary Care Research Network

Setting: Single tertiary research centre, UK

Study start date: August 2016; Study end date: July 2018

Participants

Total N: 114 in "final evaluable dataset" (145 recruited into the trial)

N per arm: Tobacco cigarettes (TC): 40; EC nicotine (16 mg): 37; EC-Nicotine-free: 37

Inclusion criteria:

- People who smoke $\geq 18$ years of age who had smoked $\geq 15$ cigarettes/day for at least 2 years 
- were free from established CV disease, diabetes, and chronic kidney disease; and were not on medication for those conditions

- Willing to stop tobacco cigarettes for period of study if required

- Willing not to use electronic cigarettes if required

- Able to give informed consent

Exclusion criteria:

- Pregnant or lactating

- Women of childbearing potential who do not abstain from sex or use effective contraception

- On current prescribed medication for cardiovascular disease

- History of cardiovascular disease (excluding hypertension), diabetes, active malignance or chronic renal disease

- Nut allergy

- Participation in another clinical trial (other than observational trials and registries) with an investigational product and/or intervention within 30 days before visit 1

65.4\% women; mean age 46.9; mean cpd 18.7

Motivated to quit: TC group: No; EC nicotine (16 mg): Yes; EC-Nicotine-free: Yes.

EC: Cig-a-like
EC nicotine (16 mg) arm: EC containing 16 mg nicotine (Vapourlites Starter Kit with XR5 16 mg nicotine
cartomizer; Vapourlites, Peterlee, United Kingdom)

EC-Nicotine-free arm: Nicotine-free EC plus nicotine flavoring (Vapourlites Starter Kit with 0 mg nicotine cartomizer)

(non-randomized) TC arm: continued their usual daily smoking habits and did not use EC for the 4week period of the trial

\begin{tabular}{|c|c|c|}
\hline Outcomes & \multicolumn{2}{|l|}{ Week 4} \\
\hline & \multicolumn{2}{|c|}{ Adverse events and biomarkers: BP, heart rate, adverse events } \\
\hline & \multicolumn{2}{|c|}{$\begin{array}{l}\text { Other outcomes measured: Endothelial function, oxidized low-density lipoprotein, high-sensitivity C- } \\
\text { reactive protein, tissue plasminogen activator, and platelet activation inhibitor-1 }\end{array}$} \\
\hline Study funding & \multicolumn{2}{|c|}{$\begin{array}{l}\text { "The VESUVIUS (Vascular Effects of Regular Cigarettes Versus Electronic Cigarette Use) trial was fund- } \\
\text { ed by the British Heart Foundation (grant PG/15/64/31681); and supported by Immunoassay Biomark- } \\
\text { er Core Laboratory, University of Dundee, the Tayside Medical Sciences Centre, and the NHS Tayside } \\
\text { Smoking Cessation Service. The funder had no role in the study design, data collection, data analysis, } \\
\text { data interpretation, writing of the report, or in the decision to submit for publication." }\end{array}$} \\
\hline Author declarations & \multicolumn{2}{|c|}{$\begin{array}{l}\text { "Dr. Donnan has received research grants from AbbVie, Shire, and Gilead Sciences. All other authors } \\
\text { have reported that they have no relationships relevant to the contents of this paper to disclose." }\end{array}$} \\
\hline Notes & \multicolumn{2}{|l|}{ New for 2020 update } \\
\hline \multicolumn{3}{|l|}{ Risk of bias } \\
\hline Bias & Authors' judgement & Support for judgement \\
\hline $\begin{array}{l}\text { Random sequence genera- } \\
\text { tion (selection bias) }\end{array}$ & Low risk & $\begin{array}{l}\text { Consented participants who were willing to quit smoking were randomized } \\
\text { to one of the EC arms in a 1:1 fashion using a centrally controlled web-based } \\
\text { good clinical practices- compliant randomization system to either: } 1 \text { ) EC con- } \\
\text { taining } 16 \mathrm{mg} \text { nicotine; or } 2 \text { ) nicotine-free EC plus nicotine flavoring because } \\
\text { it was considered by the institutional ethics committee as ethically unaccept- } \\
\text { able to randomize those who were willing to quit smoking into a smoking arm. }\end{array}$ \\
\hline
\end{tabular}




\begin{tabular}{lll}
\hline $\begin{array}{l}\text { Allocation concealment } \\
\text { (selection bias) }\end{array}$ & Low risk & Central randomization \\
\hline $\begin{array}{l}\text { Blinding of participants } \\
\text { and personnel (perfor- } \\
\text { mance bias) }\end{array}$ & Unclear risk & Not specified \\
All outcomes & \\
\hline
\end{tabular}

\begin{tabular}{|c|c|c|}
\hline \multirow{3}{*}{$\begin{array}{l}\text { Blinding of outcome as- } \\
\text { sessment (detection bias) } \\
\text { All outcomes }\end{array}$} & High risk & $\begin{array}{l}\text { Not blinded and AE/SAE data are self-report only. For other outcomes, low risk } \\
\text { as objectively measured: }\end{array}$ \\
\hline & & $\begin{array}{l}\text { Quote: "Patients fasted overnight and measurements were conducted at base- } \\
\text { line and } 1 \text { month according to the International Brachial Artery Reactivity Task } \\
\text { Force guidelines (19) by a single operator (M.H.) blinded to study allocation at } \\
\text { a single site." }\end{array}$ \\
\hline & & $\begin{array}{l}\text { "Pulse wave velocity and augmentation index were measured at baseline and } \\
1 \text { month by a single operator (M.H.) blinded to study allocation." }\end{array}$ \\
\hline
\end{tabular}

Incomplete outcome data Unclear risk
(attrition bias)

Number randomized not provided per group.

All outcomes

Quote: "A total of 145 patients were recruited into the trial (Figure 1). A final number of 114 patients (40 TC, 37 EC-nicotine, 37 EC-nicotine-free) completed both visits."

\begin{tabular}{ll}
\hline $\begin{array}{l}\text { Selective reporting (re- } \\
\begin{array}{l}\text { porting bias) } \\
\text { Low risk }\end{array}\end{array}$ & $\begin{array}{l}\text { Clinical trial record lists: Change in FMD; Change in oxidized LDL; Change in } \\
\text { PAl-1; Change in hs-CRP; Change in Pulse Wave Velocity; Change in tPA; Change } \\
\text { in Augmentation Index@75bpm }\end{array}$ \\
& All reported in the paper \\
\hline
\end{tabular}

\section{Goniewicz 2017}

\section{Study characteristics}

Design: Longitudinal within-subjects observational
Recruitment: Advertisements in the media, the internet, posted advertisements in clinics and offices,
and by word of mouth
Setting: University, Poland
Study start date: March 2011; Study end date: June 2011
Total N: 22 started out and 2 dropped out in the first week due to an adverse event (nausea) and inabili-
ty to commit to clinic visits. This resulted in analytic sample of 20
Inclusion criteria:
Participants 18 or older, current daily cigarette smokers (> 5 cpd within the last 12 months)
- May have had interest in quitting smoking, in good health (at the clinic screening visit)
- Able to communicate in Polish
- Able to use an e-cigarette safely
Exclusion criteria:


- Diagnosed as having asthma, COPD, hypertension, inhaled allergies, chronic heart disease, or cancer

- were taking a cardiac medication

- were pregnant

$60 \%$ women; mean age 31; mean cpd 16; mean FTND 3.9

Motivated to quit: At the time of screening, $95 \%$ of participants $(n=19)$ reported planning to quit smoking, with $80 \%(n=16)$ reporting that they have made at least 1 quit attempt prior to involvement in the study

E cigarette use at baseline: Not reported

Interventions

\section{EC: Cig-a-like}

Pen-style M201 e-cigarettes for 2 weeks, with an automatically-operated battery with an output power of 4.6 Volts $(280 \mathrm{mAh})$ and the heating element resistance of 3.6 - 3.8 Ohms. At baseline, provided with EC (M201 Mild, Poland) with 20 tobacco-flavored cartridges a week containing $11.0 \pm 1.5 \mathrm{mg}$ of nicotine in a mixture of propylene glycol and vegetable glycerin (50:50). Encouraged to substitute their regular cigarettes with the e-cigarette for 2 weeks and refrain from smoking

Adverse events and biomarkers:

- Biomarkers were metabolites of 13 major carcinogens and toxicants in cigarette smoke: 1 tobacco-specific nitrosamine (NNK), eight volatile organic compounds (1.3-butadiene, crotonaldehyde, acrolein, benzene, acrylamide, acrylonitrile, ethylene oxide, and propylene oxide), and 4 polycyclic aromatic hydrocarbons (naphthalene, fluorene, phenanthrene, and pyrene)

- Questionnaire on 'health': At each visit, participants were asked, "In the last week, have you experienced any of the following symptoms?", while providing a response of "never," "rarely," or "often" to the following list of health effects: daytime cough, difficulty concentrating, difficulty breathing during sleep, difficulty sleeping, dizziness, headache, irritability, nausea, nighttime cough, chest pain, phlegm, shortness of breath, tightness in chest, visual disturbances, and wheezing. Responses of "rarely" or "often" were combined to indicate presence of an adverse health effect

- Expired CO

Other outcomes measured:

- 7 nicotine metabolites (3-Hydroxycotinine, Cotinine, Cotinine N-Oxide, Nicotine N-Oxide, Norcotinine, Nornicotine, Nicotine)

- Revised Minnesota Nicotine Withdrawal Scale (MNWS-R) administered to measure 'withdrawal symptoms' (0 - 5 rating scale)

Study funding

"This work was supported by the Ministry of Science and Higher Education of Poland (grant number $\mathrm{N}$ N404 025638). Instrumentation and analytical chemistry at UCSF was supported by the National Institutes of Health, P30 DA012393 and S10 RR026437. The study sponsor had no involvement in the study design, collection, analysis, and interpretation of data, the writing of the manuscript or the decision to submit the manuscript for publication."

Author declarations

"MLG was a faculty member of the Medical University of Silesia, Poland during the study. He received a research grant from Pfizer, a pharmaceutical company that markets smoking cessation medications. MLG and NLB have been consultants to pharmaceutical companies that market smoking cessation medications. NLB has been an expert witness in litigation against tobacco companies. The other authors declare no potential conflicts of interest."

Notes New for 2020 update

\section{Risk of bias}


Goniewicz 2017 (Continued)

\begin{tabular}{lll}
$\begin{array}{l}\text { Random sequence genera- } \\
\text { tion (selection bias) }\end{array}$ & High risk & Not randomized \\
\hline $\begin{array}{l}\text { Allocation concealment } \\
\text { (selection bias) }\end{array}$ & High risk & Not randomized \\
\hline $\begin{array}{l}\text { Incomplete outcome data } \\
\text { (attrition bias) } \\
\text { All outcomes }\end{array}$ & Low risk & $\begin{array}{l}2 \text { dropouts }-1 \text { for nausea, } 1 \text { could not complete clinic visits. Analysis based on } \\
20 \text { completers }\end{array}$ \\
\hline
\end{tabular}

\begin{tabular}{ll}
\hline $\begin{array}{l}\text { Selective reporting (re- } \\
\text { porting bias) }\end{array}$ & Low risk outcomes reported \\
\hline
\end{tabular}

\section{Study characteristics}

Design: Pragmatic, open-label, single-centre, 2-arm randomized controlled trial
Recruitment: Withdrawal service in Melbourne, Australia
Setting: Substance use disorder treatment setting, and following discharge, community setting, Mel-
bourne, Australia
Study start date: 1 August 2017; Study end date: April 2019.
Total N: 100
N per arm: EC intervention = 50; NRT Control = 50
Inclusion criteria:
- Aged 18 years or over
- Tobacco smoker on entering the residential service
- Have the capacity to consent and able to understand the participant materials and follow the study
instructions and procedures (e.g. sufficient English language ability)
Exclusion criteria:
- Have used an END containing nicotine in the past month;
- Currently pregnant or breast-feeding (measured by self-report);
- Currently enrolled in another study;
- Scheduled to be transferred to a long-term rehabilitation unit following discharge from the residential
withdrawal unit.

Inclusion based on specific population characteristic: Participants were discharged from a smoke-free alcohol or other drugs (AOD) residential withdrawal service

$32 \%$ women; mean age 40.9; mean cpd 21

Motivated to quit: Median (SD) = $7.3(2.4)$ of 1 to 10 scale with 10 "highly motivated"

Up to an hours training session, information pack. Innokin Endura T22 starter kit and refill liquid (Nicophar). 4-week supply of liquid nicotine, with further supplies of liquid nicotine mailed twice at 4week intervals. Dosing schedule of e-liquid dependent nicotine dependence score: high-nicotine-dependence category assigned initial 4-week e-liquid supply (total $8 \times 10 \mathrm{ml}$ bottles) consisting of: $2 \times 10$ $\mathrm{ml}$ bottles of $18 \mathrm{mg}$ e-liquid and $6 \times 10 \mathrm{ml}$ bottles of $12 \mathrm{mg}$ e-liquid. The second and third batches $=8 \times$ 
$10 \mathrm{ml}$ bottles of $12 \mathrm{mg}$ e-liquid only. Participants scoring in the moderate- and low-dependence categories: three 4-week supplies of $8 \times 10 \mathrm{ml}$ bottles of $12 \mathrm{mg}$ e-liquid. Participants given 1-week supply of nicotine patches for use while getting used to the EC.

NRT control: Information pack, 12 weeks NRT on the same schedule as for ENDs. 4-week supply of patches plus a nicotine spray and inhaler, followed by refills including patches plus inhaler, gum and lozenges.

Both groups received proactive referral to quitline counseling (call-back service), which provides calls at pre-discharge and on days 1, 3, 7, 14 and 28 post-discharge, with an emphasis on relapse prevention. Counsellors trained on the use of ENDs.

Week 6, 12; self-report.
Adverse events collected
Other outcomes measured:
- Acceptability and feasibility of interventions
- Treatment adherence
- Cigarettes smoked per day - Heaviness of Smoking Index
- Frequency of cravings
- Minnesota Nicotine Withdrawal Scale (MNWS)
- 10 -item Kessler Psychological Distress Scale (Kessler-10)
- Quitting self-efficacy, motivation to quit and the Heaviness of Smoking Index were assessed at base-

Study funding

"The study is supported by a VicHealth Innovation Research Grant (2016-0096). AG is supported by a post-doctoral fellowship from the Heart Foundation. ALB is supported by an Australian National Health and Medical Research Council (NHMRC) senior research fellowship and a Faculty of Health and Medicine, University of Newcastle Gladys M Brawn senior research fellowship. BB is supported by an Australian NHMRC career development fellowship (GNT1063206) and a Faculty of Health and Medicine, University of Newcastle Gladys M Brawn career development fellowship."

"This study was supported by a VicHealth Innovation Research Grant (2016-0096)."

Author declarations $\quad$ "The authors declare that they have no competing interests."
"None to declare."

Notes

New for 2020 update; additional data originally provided by authors and subsequently published

\section{Risk of bias}

\begin{tabular}{lll}
\hline Bias & Authors' judgement & Support for judgement \\
\hline $\begin{array}{l}\text { Random sequence genera- } \\
\text { tion (selection bias) }\end{array}$ & Low risk & $\begin{array}{l}\text { Quote: “Upon completing the baseline survey, participants were randomised } \\
1: 1 \text { to an intervention via a computer-sequenced 4-6 block randomisation em- } \\
\text { bedded in the tablet device software." }\end{array}$ \\
\hline $\begin{array}{l}\text { Allocation concealment } \\
\text { (selection bias) }\end{array}$ & Low risk & $\begin{array}{l}\text { Quote: "At the end of the baseline survey, participants will be randomised 1:1 } \\
\text { to an intervention via a computer-sequenced 4-6 block randomisation em- } \\
\text { bedded in the iPad." }\end{array}$ \\
$\begin{array}{l}\text { Blinding of participants } \\
\text { and personnel (perfor- } \\
\text { mance bias) } \\
\begin{array}{l}\text { All outcomes } \\
\text { bigh risk }\end{array}\end{array}$ & $\begin{array}{l}\text { Quote: "Participants were informed of their intervention group by the RA and } \\
\text { provided with a training session of up to one hour." } \\
\text { "Due to the nature of the intervention, neither participants nor staff can be } \\
\text { blinded to allocation. However, the data safety monitoring committee and the } \\
\text { statistician responsible for the data analysis will be blinded." }\end{array}$ \\
\hline \hline
\end{tabular}


Guillaumier 2018 (Continued)
Blinding of outcome as-
High risk
No biochemical validation, self-report data sessment (detection bias)

All outcomes

\begin{tabular}{|c|c|c|}
\hline $\begin{array}{l}\text { Incomplete outcome data } \\
\text { (attrition bias) } \\
\text { All outcomes }\end{array}$ & Low risk & $\begin{array}{l}\text { Quote: "At } 6 \text { and } 12 \text {-weeks, } 63 \text { participants ( } 63 \% \text { ) and } 50 \text { participants }(50 \%) \\
\text { were followed up, respectively. While slightly higher retention rates were evi- } \\
\text { dence in the VNP group at } 6 \text {-weeks ( } 68 \% \text { vs } 58 \% \text { in NRT group; } p=0.300) \text {; there } \\
\text { were no differences between groups at } 12 \text {-weeks ( } 25 \text { recontacted in both } \\
\text { arms; i.e., } 50 \%) \text {." }\end{array}$ \\
\hline $\begin{array}{l}\text { Selective reporting (re- } \\
\text { porting bias) }\end{array}$ & Low risk & $\begin{array}{l}\text { Unpublished findings provided by authors report on all outcomes mentioned } \\
\text { in the protocol }\end{array}$ \\
\hline
\end{tabular}

\section{Hajek 2015a}

\section{Study characteristics}

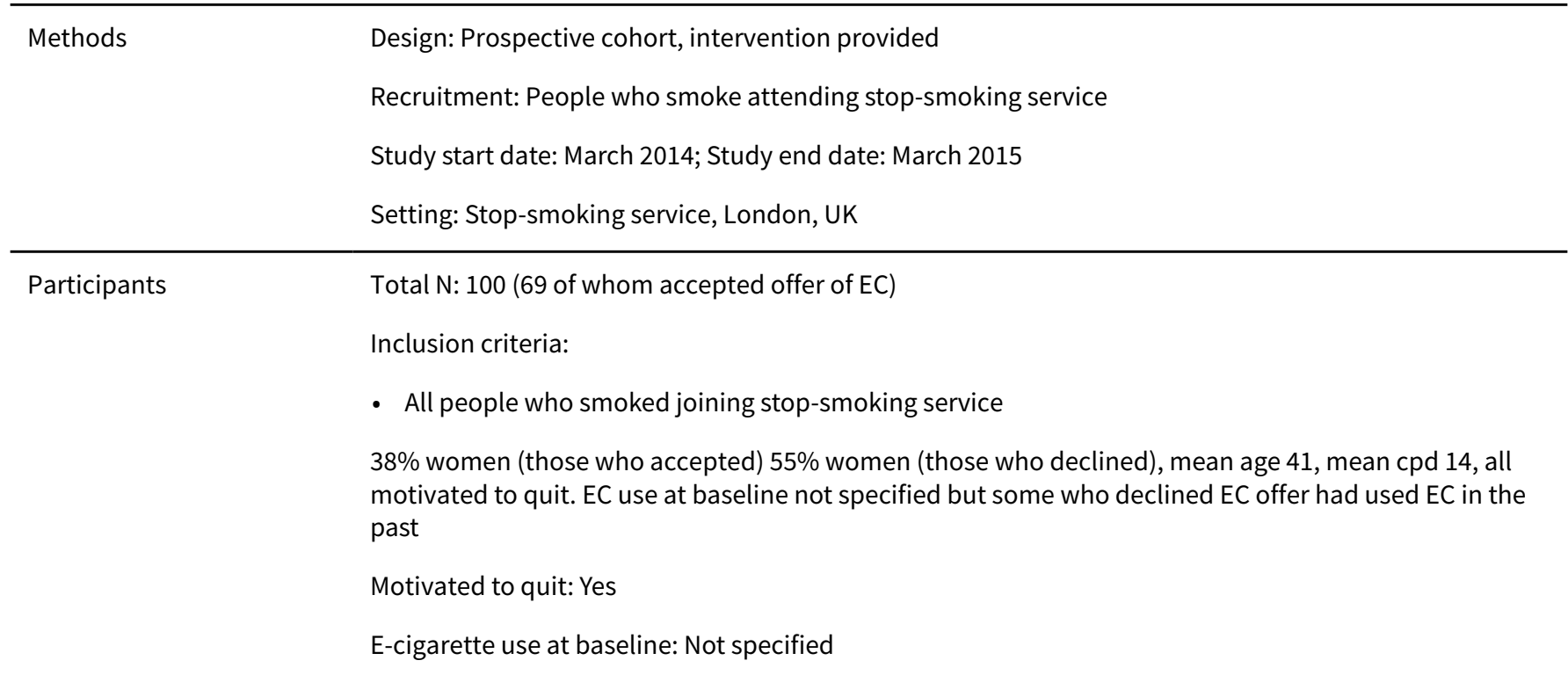

Interventions

\section{EC: Cig-a-like and refillable}

EC: offered to all people who smoke joining service; offered choice of 'cig-a-like' (Gamucci, $1.6 \%$ or $2.2 \%$ nicotine per $\mathrm{ml}$ ) product or tank model (EVOD, $1.8 \%$; later replaced with Aspire product due to leakage issues). $69 \%$ of those offered received an EC on TQD

Medication: Offered stop-smoking medications including NRT and varenicline as in standard protocol. Of EC users $33 \%$ opted to also use NRT, $29 \%$ varenicline, $38 \%$ nothing

Support: weekly, as in standard protocol

\begin{tabular}{ll}
\hline Outcomes & Adverse events collected throughout, method for collection unclear \\
& Also collected: 4-week biochemically-validated abstinence, participant feedback, cost \\
\hline Study funding & "The pilot study was sponsored by City of London Corporation." \\
\hline Author declarations & $\begin{array}{l}\text { "Peter Hajek received research funds from and provided consultancy to manufacturers of smoking ces- } \\
\text { sation medications. The remaining authors have no conflicts of interest to declare." }\end{array}$
\end{tabular}


Hajek 2015a (Continued)

Notes

\section{Risk of bias}

\begin{tabular}{lll}
\hline Bias & Authors' judgement & Support for judgement \\
\hline $\begin{array}{l}\text { Random sequence genera- } \\
\text { tion (selection bias) }\end{array}$ & High risk & Not randomized \\
\hline $\begin{array}{l}\text { Allocation concealment } \\
\text { (selection bias) }\end{array}$ & High risk & Not randomized \\
\hline $\begin{array}{l}\text { Incomplete outcome data } \\
\text { (attrition bias) } \\
\text { All outcomes }\end{array}$ & Unclear risk & $\begin{array}{l}\text { 26\% lost in EC group, dropout rate in EC decliners not reported. Reasons for } \\
\text { dropout not stated }\end{array}$ \\
\hline $\begin{array}{l}\text { Selective reporting (re- } \\
\text { porting bias) }\end{array}$ & Unclear risk & Unclear which outcomes authors set out to collect, no protocol available \\
\hline
\end{tabular}

Hajek 2019

\section{Study characteristics}

\begin{tabular}{l} 
Methods \\
pared with nicotine replacement therapy \\
Recruitment: participants attending UK stop-smoking service and via social media \\
Setting: U.K. National Health Service stop-smoking services \\
Study start date: 1 April 2015; Study end date: 31 March 2018 \\
\hline Total N: 886 \\
N per arm: EC: $439 ;$ NRT: 447 \\
Inclusion criteria: \\
- Adults who smoke (aged 18 or over) with no strong preference to use or not to use nicotine replace- \\
ment or e-cigarettes, and were currently not using either type of product \\
- Able to read/write/understand English \\
Exclusion criteria: \\
- Pregnant or breastfeeding \\
- Strong preference to use or not use NRT or EC, currently not using either type of product \\
$48 \%$ women; median age $41 ;$ median cpd 15 ; mean FTND $4.6 ; 41.5 \%$ reported past use of ECs \\
Motivated to quit: Not reported \\
\hline
\end{tabular}

Interventions

\section{EC: Refillable}

NRT: Informed of range of NRT products and selected preferred product, encouraged to use combination. Participants free to switch products. Supplies provided for up to 3 months

EC: Starter pack (1 Kit, Aspire UK) provided along with $30 \mathrm{ml}$ bottle of Tobacco Royale flavor e-liquid, concentration $18 \mathrm{mg} / \mathrm{ml}$. Participants showed how to use and asked to purchase future e-liquid online or from local vape shops and to buy different EC device if the 1 provided did not meet their needs. 
Hajek 2019 (Continued)

Enouraged to experiment with e-liquids of different strengths and flavors. If unable to obtain own supply, provided with further 10-ml bottle (not proactively offered). Oral and written info on how to operate EC

Both arms received multi-session behavioral support as per UK stop-smoking service practice (one-toone sessions weekly with local clinicians, exhaled CO monitored for at least 4 weeks post-TQD); signed behavioral contract not to use other therapy for at least 4 weeks

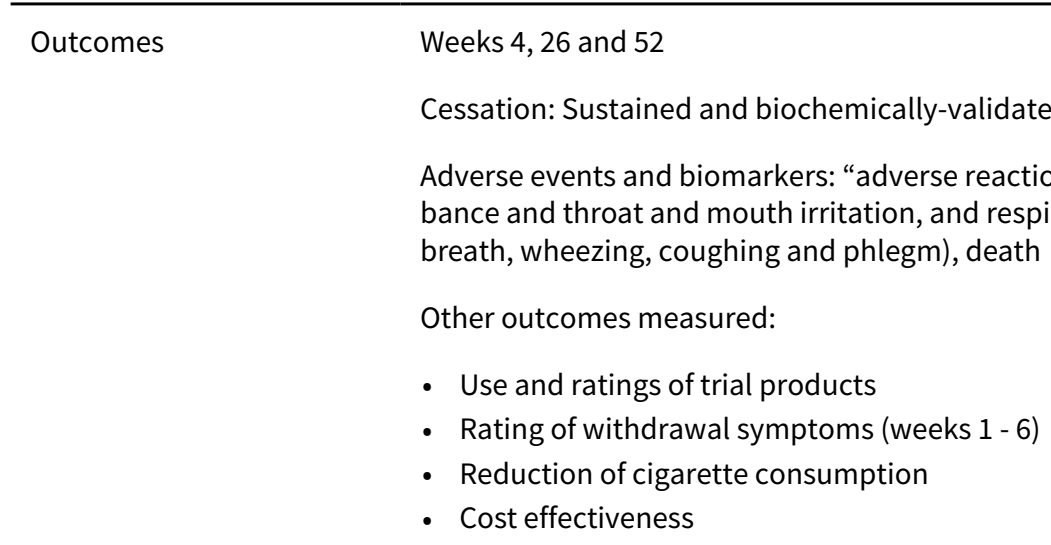

Study funding

"Supported by the National Institute for Health Research (NIHR) Health Technology Assessment Programme (project number, 12/167/135) and by a grant (A16893) from the Cancer Research UK Prevention Trials Unit."

Author declarations

From ICJME disclosure forms: "Miss Natalie Bisal has nothing to disclose. Dr. Dawkins reports personal fees from Johnson \& Johnson, outside the submitted work; Dr. Goniewicz reports personal fees from Johnson and Johnson, outside the submitted work; Dr. Hajek reports grants and personal fees from Pfizer, outside the submitted work; Ms. Li reports grants from NCCHTA, during the conduct of the study; Dr. McRobbie reports grants from NIHR HTA program, during the conduct of the study; personal fees from Pfizer, personal fees from Johnson \& Johnson, outside the submitted work; Dr. Myers Smith has nothing to disclose. Dr. Parrott has nothing to disclose. Dr. Pesola has nothing to disclose. Mrs Anna Phillips-Waller has nothing to disclose. Dr. Przulj reports grants from Pfizer, outside the submitted work; Dr. Ross has nothing to disclose. Dr. Sasieni has nothing to disclose. Ms. Wu has nothing to disclose."

$\begin{array}{ll}\text { Notes } & \text { New for } 2020 \text { update, listed as ongoing study ISRCTN60477608 in } 2016 \text { review update } \\ & \text { Note higher use of allocated product at } 12 \mathrm{~m} \text { in intervention group compared to control group: "Among } \\ \text { participants with 1-year abstinence, } 80 \% \text { (63 of 79) were using e-cigarettes at } 52 \text { weeks in the e-ciga- } \\ \text { rette group and } 9 \% \text { (4 of 44) were using nicotine replacement in the nicotine-replacement group." }\end{array}$

\section{Risk of bias}

\section{Bias \\ Authors' judgement Support for judgement}

Random sequence genera- Low risk tion (selection bias)
Quote: "Randomization took place on the quit date to limit differential dropout. Randomization sequences (1:1 ratio in permuted blocks of 20, stratified according to trial site) were generated with the use of a pseudorandom number generator in Stata software and were embedded into an application that only revealed the next treatment assignment once a participant had been entered into the database."

$\begin{aligned} & \text { Allocation concealment } \\ & \text { (selection bias) }\end{aligned}$ Low risk Refer to 'Random sequence generation'.


Hajek 2019 (Continued)

Blinding of participants Low risk Not blinded, but as both arms contained active interventions performance and personnel (perforbias judged unlikely mance bias)

All outcomes

\begin{tabular}{lll}
\hline Blinding of outcome as- & Low risk & Biochemical validation used \\
sessment (detection bias) &
\end{tabular}

All outcomes

Incomplete outcome data Low risk

(attrition bias)

All outcomes

At 12 months:

EC Arm: 356/439

NRT Arm: 342/447

Selective reporting (re- Low risk All prespecified outcomes reported

porting bias)

\section{Halpern 2018}

\section{Study characteristics}

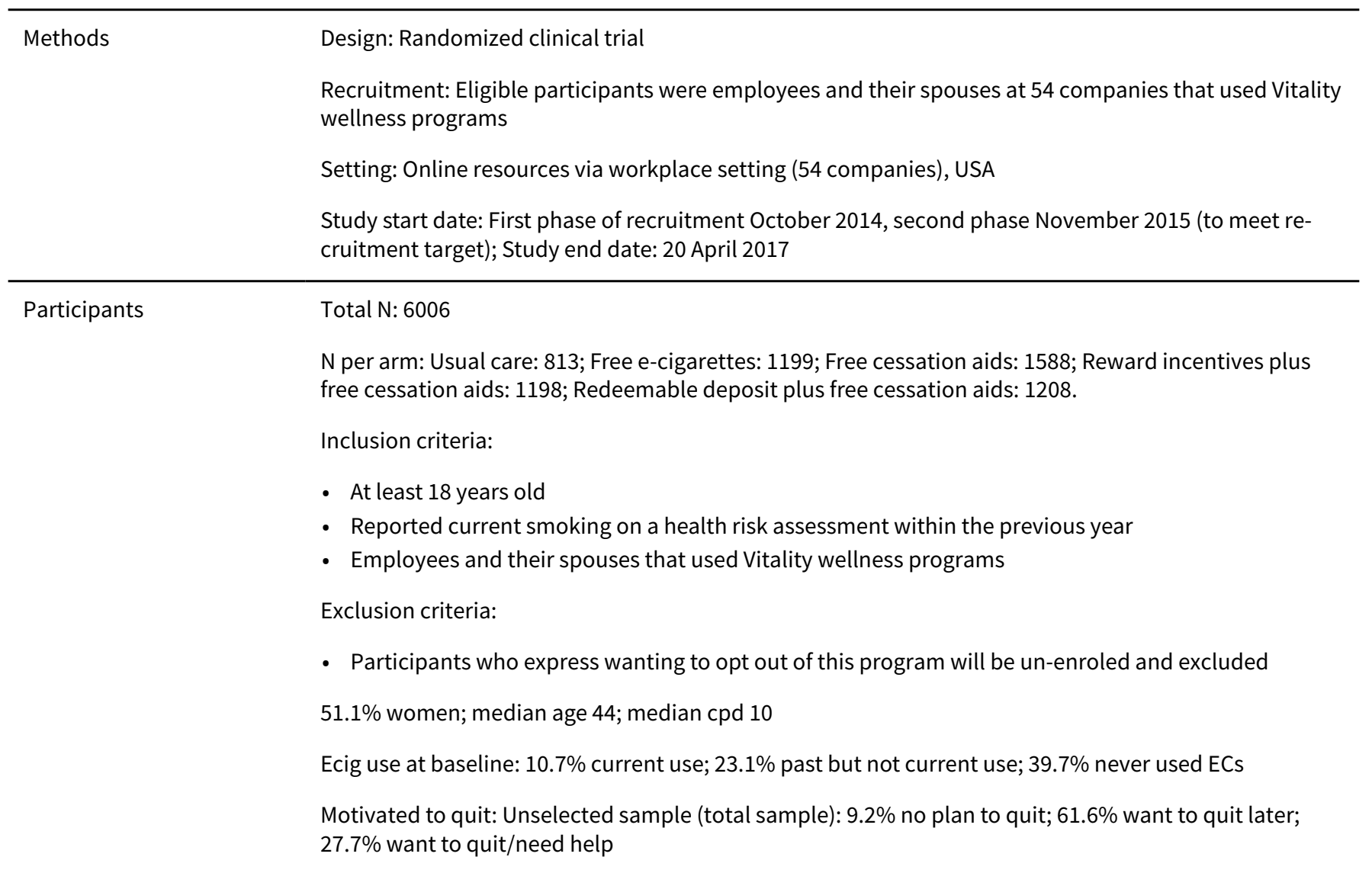

Interventions

\section{EC: Cig-a-like}

\section{a) Usual care:}

Standardized Vitality program aimed at promoting tobacco cessation. This program includes existing employee benefits for quitting and the use of text/email messages to encourage tobacco cessation 
Halpern 2018 (Continued)

b) as (a), plus free EC:

Free NJOY e-cigarettes (including battery sticks, a USB charger, and up to 20 chambers with 1.0 to $1.5 \%$ nicotine per week in participants' chosen flavors). Use of all products was free until 6 months after the quit date

c) as (b) plus access to free NRT, bupropion or varenicline

d) as (c) plus incentives across $6 \mathrm{~m}$ for testing negative for tobacco use

e) as (c) plus provide money at start and lose money from this fund if they do not test negative across 6 $\mathrm{m}$
Notes

New for 2020 update. Study listed as ongoing study NCT02328794 in 2016 review update

Only arms (a) and (b) included in our analyses.

\section{Risk of bias}

\begin{tabular}{|c|c|c|}
\hline Bias & Authors' judgement & Support for judgement \\
\hline $\begin{array}{l}\text { Random sequence genera- } \\
\text { tion (selection bias) }\end{array}$ & Unclear risk & Not specified \\
\hline $\begin{array}{l}\text { Allocation concealment } \\
\text { (selection bias) }\end{array}$ & Unclear risk & Not specified \\
\hline $\begin{array}{l}\text { Blinding of participants } \\
\text { and personnel (perfor- } \\
\text { mance bias) } \\
\text { All outcomes }\end{array}$ & High risk & Not blinded and different amounts of support given to each group \\
\hline $\begin{array}{l}\text { Blinding of outcome as- } \\
\text { sessment (detection bias) } \\
\text { All outcomes }\end{array}$ & Low risk & Biochemical validation \\
\hline $\begin{array}{l}\text { Incomplete outcome data } \\
\text { (attrition bias) } \\
\text { All outcomes }\end{array}$ & High risk & $\begin{array}{l}\text { At } 12 \text { months very low numbers completed biochemical validation. Submitted } \\
\text { a sample } n=C G: 1 \text {, free e-cigs; } 4 \text {, free cessation:5, rewards: } 14 \text {, deposits: } 16\end{array}$ \\
\hline $\begin{array}{l}\text { Selective reporting (re- } \\
\text { porting bias) }\end{array}$ & Low risk & Expected outcomes reported and checked with trial registration \\
\hline
\end{tabular}




\section{Study characteristics}

Methods Design: randomized trial

Recruitment: Media advertisements

Setting: Clinic visits in community, USA

Study start date: 25 November 2014; Study end date: 2 December 2018

\section{Participants}

Total N: 264

N per arm: Usual brand: 36; AD-E: 76; CS-E: 76; CS-NRT: 76.

Inclusion criteria:

- At least 18 years of age

- Smoking at least $5 \mathrm{cpd}$ for the past year with a breath $\mathrm{CO}$ at least $10 \mathrm{ppm}$ or NicAlert test $=$ level 6 if CO less than $10 \mathrm{ppm}$

- In stable physical and mental health

Exclusion criteria:

- A serious quit attempt in the past 3 months

- Recent (<3 months) alcohol or drug abuse problems

- Regular use of other nicotine or tobacco products (e.g. > 9 days per month to minimize confounding effects of these products on biomarker outcomes)

- Planning to quit smoking in the next 3 months

- Chronic conditions affecting results of biomarker analyses (e.g., liver disease)

- Currently using NRT or other cessation medications

- Pregnant, planning to become pregnant, or breastfeeding

49\% women; mean age 45.2; mean cpd 15.2; mean FTND 3.4

E cigarette use at baseline: Not reported

Motivated to quit: Initially uninterested

Interventions

EC: Cig-a-like, but the only cig-a-like product with high nicotine content

Usual brand arm: Purchased their own usual brand of cigarettes; at end of clinical trial phase (week 8), offered ECs or NRT for up to 8 weeks, with a choice of product and no specific instructions for use

EC AD-E arm: Use EC whenever you like instead of a cigarette; can smoke as many or as few cigarettes as you want

EC CS-E arm: Complete substitution with e-cigarettes (i.e. "you will stop smoking cigarettes and use only e-cigarettes")

The primary e-cigarette product was Vuse Solo ( $4.8 \%$ nicotine, manufactured by RJ Reynolds, Inc). Initially a choice of Blu cigarettes (cartridge-based system, marketed previously by Lorillard) and Fin (prefilled tanks system, manufactured by Fin Branding Group) was offered; but because Vuse attained the highest market share during the early phase of the study, switched exclusively to Vuse. Participants could choose 1 of 4 flavors: tobacco, mint, menthol, and berry. Participants were provided 7 cartridges a week with the option of returning to the clinic before their next visit to obtain additional cartridges if needed. All products provided free to the participants. All unused products and used EC cartridges were collected at each visit

CS-NRT arm: Complete substitution with $4 \mathrm{mg}$ nicotine gum or lozenge, with the participant choosing what product they would like to use (i.e. "you will stop smoking cigarettes and use only nicotine gum 
Hatsukami 2020 (Continued)

or lozenge"). The $4 \mathrm{mg}$ was down-titrated to $2 \mathrm{mg}$ if adverse side effects were experienced. Nicotine gum came in mint, cinnamon, and fruit flavors, while the nicotine lozenge was mint or cherry flavors. All these products were provided free to the participants and unused products were collected at each visit

Behavioural support: CS-E arm and CS-NRT arm: received brief counseling on how to avoid smoking cigarettes

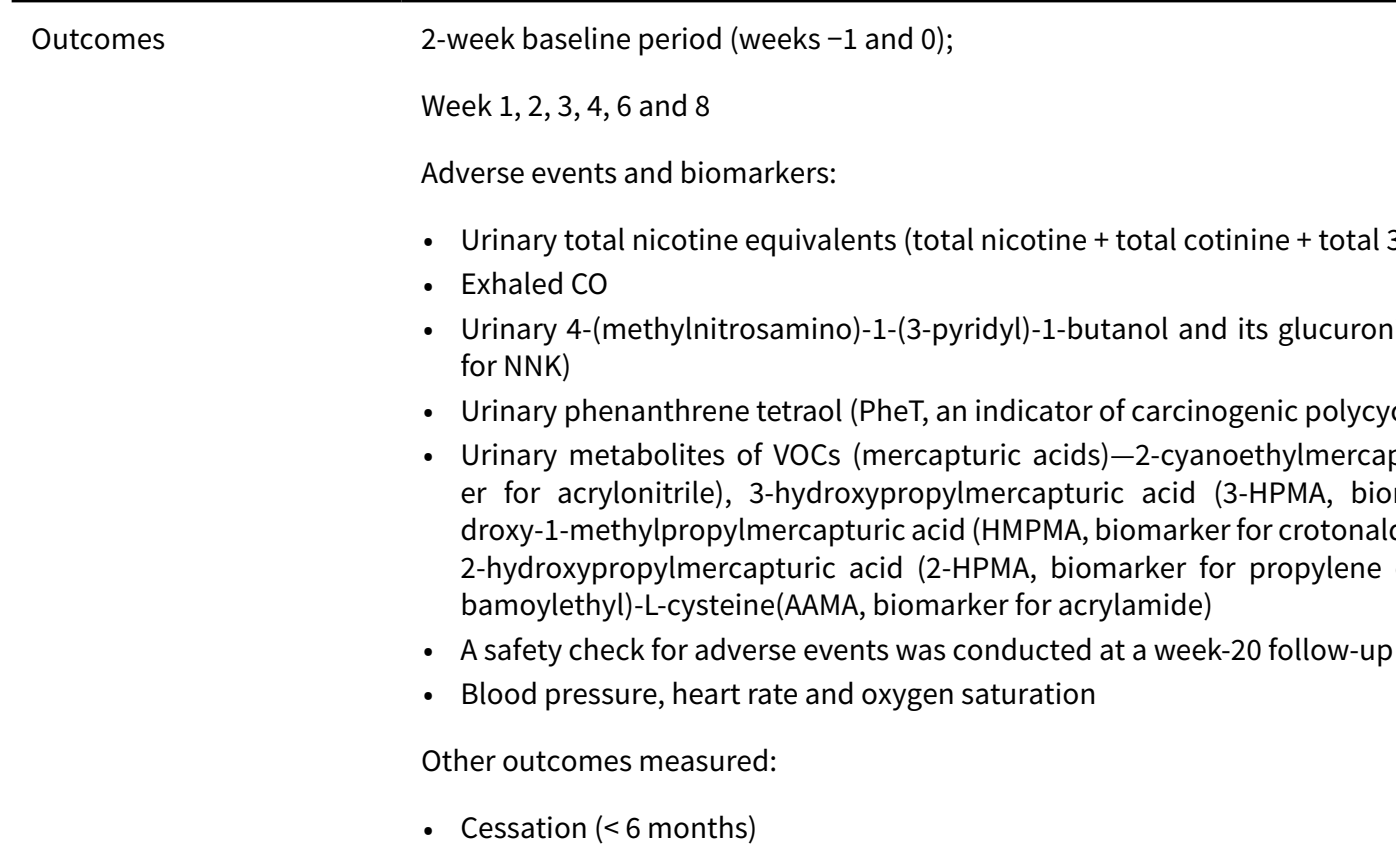

Study funding

"supported by grants U19CA157345 from the National Cancer Institute (DKH/PS), UL1 TR000062 and UL1 TR002494 from the National Center for Advancing Translational Science of the National Institutes of Health, and T32 DA007097 from the National Institute of Drug Abuse (EM). The content is solely the responsibility of the authors and does not necessarily represent the official views of the funding agencies"

Author declarations

"RJC is a member of the FDA Tobacco Products Scientific Advisory Committee. PGS serves or has served as an expert witness in tobacco company litigation on behalf of plaintiffs"

Notes New for 2020 update. AD-E arm not included in this review

Additional data provided from authors.

\section{Risk of bias}

\begin{tabular}{lll}
\hline Bias & Authors' judgement & Support for judgement \\
\hline $\begin{array}{l}\text { Random sequence genera- } \\
\text { tion (selection bias) }\end{array}$ & Unclear risk & Not specified \\
\hline $\begin{array}{l}\text { Allocation concealment } \\
\text { (selection bias) }\end{array}$ & Unclear risk & Not specified \\
\hline $\begin{array}{l}\text { Blinding of participants } \\
\text { and personnel (perfor- } \\
\text { mance bias) }\end{array}$ & Unclear risk & Not blinded and some interventions contained different levels of support \\
All outcomes & \\
\hline
\end{tabular}


Hatsukami 2020 (Continued)

Blinding of outcome as- Low risk Not blinded but all relevant outcomes for our analyses were objective sessment (detection bias) All outcomes
Incomplete outcome data Low risk (attrition bias)

All outcomes
Quote: "There was a significant difference in dropout rates across groups following study entry $(p=.041)$, with the highest dropout rates observed in the complete substitution groups, particularly in the NRT group..."

AD-E: Week $1=73 / 76 ;$ Week $2=73 / 76 ;$ Week $4=69 / 76 ;$ Week $6=66 / 76 ;$ Week $8=$ $65 / 76=85 \%$

CS-E: Week 1 =69/76; Week 2 =67/76; Week 4 =66/76; Week 6 =61/76; Week 8 = $58 / 76=69.7 \%$

CS-NRT: Week $1=72 / 76$; Week $2=65 / 76 ;$ Week $4=60 / 76$; Week $6=57 / 76 ;$ Week $8=53 / 76=69.7 \%$

UB: Week $1=35 / 36 ;$ Week $2=35 / 36 ;$ Week $4=33 / 36 ;$ Week $6=33 / 36 ;$ Week $8=$ $32 / 36=88.8 \%$

Selective reporting (re- Low risk porting bias)
Table in supplementary section describes that heart rate, blood pressure and oxygen levels were measured, but findings not reported in paper; however, provided by authors upon request

Hickling 2019

\section{Study characteristics}

Methods

Design: Single-group assignment - pre-test post-test pilot study

Recruitment: Participants were referred from community mental health teams within the South London and Maudsley NHS Foundation Trust.

Setting: Healthcare setting, UK.

Study start date: 24 September 2014; Study end date: 2 May 2017

\section{Participants}

Total N: 50

Inclusion criteria:

- Aged 18-70 years;

- Daily smoker (unwilling to quit soon);

- Exhaled CO level of more than five parts per million;

- An established clinical diagnosis of schizophreniform, schizophrenia, schizoaffective disorder or bipolar disorder, or attending an early detection service in a high-risk state

Exclusion criteria:

- The use of e-cigarettes on more than two occasions in the past 30 days;

- Intention to quit smoking in the next 30 days;

- Medication use that may reduce smoking (including, bupropion, nicotine replacement therapies, acamprosate, varenicline, baclofen, clonidine, naltrexone, buprenorphine, nortriptyline, disulfiram and anti-seizure medications)

- Hospitalisation/change in dose of psychotropic medication(s) in the last 30 days;

- Unstable physical health in the past 3 months;

- A previous serious stomach ulcer and/or phaeochromocytoma 
- Severe heartburn, stroke, unstable kidney/liver disease, an uncontrolled overactive thyroid gland in the past 3 months;

- Individuals who meet the Diagnostic and Statistical Manual of Mental Disorders, Fourth Edition (DSMIV) criteria for illicit/alcohol drug dependency;

- Medical contraindications to nicotine;

- Asthma

- Suicidal ideation/suicide attempt in the past month

- Pregnancy

Inclusion based on specific population characteristic: People who smoke tobacco with a psychotic disorder (established clinical diagnosis of schizophreniform, schizophrenia, schizoaffective disorder or bipolar disorder, or attending an early detection service in a high-risk state)

24\% women; mean age 38.96; mean cpd 17.94; mean FTND not reported

Motivated to quit: "unwilling to quit soon"

E-cigarette use at baseline: Must not have used e-cigarettes on more than 2 occasions in the past 30 days

Interventions

\section{EC: Cig-a-like}

Participants provided with free tobacco-flavored NJOY traditional bold disposable e-cigarette (4.5\% nicotine) in an "amount equivalent to $150 \%$ of their daily tobacco use (as recommended by the manufacturer)" for 6 weeks. Participants were instructed in the use EC; not required to stop smoking tobacco, but were encouraged to replace it with EC as much as possible. Followed up at 4 weeks and encouraged to continue EC use, informed about EC types and where these could be purchased

\section{Outcomes}

Weeks 1, 2, 3, 4, 5, 6, 7, 8, 9, 10, 24
Self-reported and biochemical validation

Cessation: Tobacco use, as measured by the Time Line Follow Back. Tobacco cigarette use was also indexed weekly by measuring exhaled CO levels with a Smokerlyzer ED50 CO meter (Bedfont Instruments, UK)

\section{Adverse events and biomarkers:}

- Side effects associated with e-cigarette use - reported weekly

- Respiratory symptoms: lung capacity (measured by Wright's Mini Peak-flow Meter (Clement Clarke International Ltd., UK) at baseline, weeks 6, 10 and 24; Peak flow was obtained 3 times at each assessment

- Heart rate and blood pressure

- Occurrence of (serious) adverse events was assessed on a weekly basis

In a subsample of participants ( $\mathrm{N}=8$ ), 3-hydroxypropylmercapturic acid (3-HPMA, a measure of the toxicant acrolein) and formic acid were measured at baseline and week 6 . These participants were chosen as their tobacco intake had decreased by more than $50 \%$ in this period. The measurement of 3-HPMA and formic acid was also performed by validated LC-MS/MS assays

Other outcomes measured:

- Urinary cotinine

- Weight

- Motivation to Stop Scale (MTSS)

- Smoking Consequences Questionnaire-Adult (SCQ-A)

- Positive and Negative Syndrome Scale (PANSS)

- Calgary Depression Scale for Schizophrenia (CDSS) 
Hickling 2019 (Continued)

Study funding

"This work was funded by the Maudsley Charity (grant number 715); and supported by the National Institute for Health Research (NIHR) Biomedical Research Centre at South London and Maudsley NHS Foundation Trust and King's College London."

$\begin{array}{ll}\text { Author declarations } & \text { "R.P-I. has received honoraria and speaker support from Lundbeck. L.D. has provided consultancy for } \\ \text { the pharmaceutical industry (Johnson \& Johnson 2015, 2017) and acted as an expert witness for an } \\ \text { e-cigarette patent infringement case (Porzio, Bromberg \& Newman Attorneys at Law, 2015). Between } \\ 2011 \text { and 2013, she conducted research for several independent electronic cigarette companies (Total- } \\ \text { ly Wicked, SKYCIGS and E-Lites) for which the University of East London received funds. The e-cigarette } \\ \text { companies involved had no input into the design, conduct or write up of these projects and she has not } \\ \text { received any funds from e-cigarette companies in the last } 4 \text { years. She has no links with, and has not } \\ \text { received any funds from, the tobacco industry, although two e-cigarette companies that she worked } \\ \text { with in } 2013 \text { were subsequently acquired by the tobacco industry (SKYCIGs and E-Lites). L.H., T.R., K- } \\ \text { V.S., J.M., A.M. and P.M. have no conflicts of interest." }\end{array}$

Notes Study listed as ongoing study NCT02212041 in the 2016 review update

Additional data provided from authors

\section{Risk of bias}

\begin{tabular}{lll}
\hline Bias & Authors' judgement & Support for judgement \\
\hline $\begin{array}{l}\text { Random sequence genera- } \\
\text { tion (selection bias) }\end{array}$ & High risk & Uncontrolled study \\
\hline $\begin{array}{l}\text { Allocation concealment } \\
\text { (selection bias) }\end{array}$ & High risk & Uncontrolled study \\
\hline $\begin{array}{l}\text { Incomplete outcome data } \\
\begin{array}{l}\text { (attrition bias) } \\
\text { All outcomes }\end{array}\end{array}$ & Low risk & Follow-up: Week 6: 46/50; Week 10: 42/50; Week 24: 40/50 \\
\hline $\begin{array}{l}\text { Selective reporting (re- } \\
\text { porting bias) }\end{array}$ & Low risk & $\begin{array}{l}\text { Report all outcomes listed on http://clinical trials.gov except NNAL. Authors } \\
\text { confirmed that they had intended to test for NNAL but had major issues with } \\
\text { the assays }\end{array}$ \\
\hline
\end{tabular}

Holliday 2019

\section{Study characteristics}

$\begin{array}{ll}\text { Methods } & \text { Design: Pilot RCT } \\ & \text { Recruitment: Recruited via the Newcastle Dental Hospital and by primary care practitioners working in } \\ \text { the north-east England region } \\ \text { Setting: Dental clinical research facility (DCRF), located in the Newcastle Dental Hospital, Newcastle } \\ \text { upon Tyne, UK. } \\ \text { Study start date: } 20 \text { September 2016; Study end date: } 31 \text { July } 2018 \\ \text { Total N: } 80 \\ \text { N per arm: Intervention group: 40; Control group: } 40 \\ \text { Inclusion criteria: } \\ \text { - Aged over } 18 \text { years old; smoker ( } \geq 10 \text { cigarettes/day) }\end{array}$


Holliday 2019 (Continued)

- Willing and able to come to the DCRF for the required study visits

- Having a minimum of 16 natural teeth (excluding third molars)

- Being diagnosed with periodontitis

Exclusion criteria:

- Having used an e-cigarette for more than 2 days in the last 30 days

- Infectious or systemic diseases that may be unduly affected by participation in this study

- Haemodynamically unstable

- Patients taking the medication adenosine (due to drug interaction risk)

- Lack of capacity to be able to consent to the research project or inability to follow study instructions, or both

- Participation in a dental research study within the previous 20 days

- Pregnant by medical history, or nursing

- Received any non-surgical periodontal therapy other than a routine scale and polish in the last 6 months

- Currently undergoing or requiring extensive dental, orthodontic or implant treatment, or treatment for peri-implantitis

Inclusion based on specific population characteristic: Periodontitis

52.5\% women; mean age 44.36; mean cpd 17.4; mean FTND 5

Motivated to quit: Not selected on motivation and not reported

E-cigarette use at baseline: Not currently using an e-cigarette, or not having used 1 for more than 2 days in the last 30 days

\section{EC: Refillable}

All participants given standard stop-smoking advice (10 - 15 minutes in duration) and offer of referral to stop-smoking services

Intervention: given EC starter kit (Vype eTank clearomizer) and brief training on its use by a dentist. Provided with an approximately 2-week supply of e-liquid $(20 \mathrm{ml}$ ) with a choice of flavor (Blended Tobacco, Crisp Mint, Dark Cherry and Vpure (flavorless)) and nicotine strength $(0 \mathrm{mg} / \mathrm{ml}, 6 \mathrm{mg} / \mathrm{ml}, 12 \mathrm{mg}$ / $\mathrm{ml}, 18 \mathrm{mg} / \mathrm{ml}$ ) and information on where to buy more. EC intervention delivered directly following the standard stop-smoking advice and was expected to be 10 - 15 minutes in duration

Control group: no further intervention
Months 1 and 6; Self-report and biochemical validation of smoking status

Cessation: Rates of continuous eCO-verified smoking abstinence at 6 months were calculated following the Russell Standard (RS6)

Adverse events and biomarkers: expired air CO, adverse events monitored at each study visit

Other outcomes measured:

- Feasibility outcomes

- Oral health outcomes

- Smoking behavior outcomes comprised: self-reported tobacco and e-cigarette use, eCO, e-salivary cotinine (SC), salivary anabasine (SA), FTND and Mood and Physical Symptoms Scale (MPS)
"Richard Holliday is funded by a National Institute for Health Research Doctoral Research Fellowship (DRF-2015-08-077). This paper presents independent research funded by the National Institute for Health Research (NIHR). The views expressed are those of the authors and not necessarily those of the NHS, the NIHR or the Department of Health and Social Care." 
Holliday 2019 (Continued)

Author declarations The authors declare that they have no competing interests."

Notes New for 2020 update.

\section{Risk of bias}

\begin{tabular}{|c|c|c|}
\hline Bias & Authors' judgement & Support for judgement \\
\hline $\begin{array}{l}\text { Random sequence genera- } \\
\text { tion (selection bias) }\end{array}$ & Low risk & $\begin{array}{l}\text { Randomization was performed using a secure password-protected web-based } \\
\text { system }\end{array}$ \\
\hline $\begin{array}{l}\text { Allocation concealment } \\
\text { (selection bias) }\end{array}$ & Low risk & $\begin{array}{l}\text { Quote: "The randomisation allocation schedule will be generated by a statisti- } \\
\text { cian with no other involvement in the study to achieve concealment of alloca- } \\
\text { tion." }\end{array}$ \\
\hline $\begin{array}{l}\text { Blinding of participants } \\
\text { and personnel (perfor- } \\
\text { mance bias) } \\
\text { All outcomes }\end{array}$ & High risk & $\begin{array}{l}\text { Nature of study precluded blinding; different levels of support across interven- } \\
\text { tion arms }\end{array}$ \\
\hline $\begin{array}{l}\text { Blinding of outcome as- } \\
\text { sessment (detection bias) } \\
\text { All outcomes }\end{array}$ & Low risk & Biochemical validation \\
\hline $\begin{array}{l}\text { Incomplete outcome data } \\
\text { (attrition bias) } \\
\text { All outcomes }\end{array}$ & Low risk & Attrition $<50 \%$ \\
\hline $\begin{array}{l}\text { Selective reporting (re- } \\
\text { porting bias) }\end{array}$ & Low risk & All prespecified outcomes are reported \\
\hline
\end{tabular}

Humair 2014

\section{Study characteristics}

\begin{tabular}{ll}
\hline Methods & Design: Prospective cohort \\
& Recruitment: People attending an outpatient clinic \\
& Setting: University hospital outpatient clinic, Switzerland \\
& Study start date/end date: Not specified \\
\hline Participants & Total N: 17 \\
Inclusion criteria: & Wish to reduce tobacco use or had failed to stop smoking using varenicline, bupropion or NRT in past \\
Inclusion based on specific population characteristic: No \\
Mean 23 cpd, $82 \%$ had a psychiatric illness \\
Motivated to quit: Yes \\
E-cigarette use at baseline: Not specified \\
EC: Cig-a-like
\end{tabular}


Humair 2014 (Continued)

Offered an EC with nicotine

$59 \%$ also reported using NRT or varenicline in addition to EC

\begin{tabular}{|c|c|c|}
\hline \multirow[t]{3}{*}{ Outcomes } & \multicolumn{2}{|c|}{ Smoking cessation and reduction by at least $30 \%$ at 12 months (self-report) } \\
\hline & \multicolumn{2}{|l|}{ Adverse events } \\
\hline & \multicolumn{2}{|c|}{ No significant side effects } \\
\hline Study funding & \multicolumn{2}{|l|}{ Not specified } \\
\hline Author declarations & \multicolumn{2}{|l|}{ Not specified } \\
\hline Notes & \multicolumn{2}{|c|}{ Abstract only, hence little detail available } \\
\hline & \multicolumn{2}{|c|}{ Not clear if EC was provided by clinic or if participants had to buy their own } \\
\hline \multicolumn{3}{|l|}{ Risk of bias } \\
\hline Bias & Authors' judgement & Support for judgement \\
\hline $\begin{array}{l}\text { Random sequence genera- } \\
\text { tion (selection bias) }\end{array}$ & High risk & Prospective cohort \\
\hline $\begin{array}{l}\text { Allocation concealment } \\
\text { (selection bias) }\end{array}$ & High risk & Not randomized \\
\hline $\begin{array}{l}\text { Incomplete outcome data } \\
\text { (attrition bias) } \\
\text { All outcomes }\end{array}$ & Unclear risk & Numbers lost to follow-up not reported \\
\hline $\begin{array}{l}\text { Selective reporting (re- } \\
\text { porting bias) }\end{array}$ & Unclear risk & Unable to determine prespecified outcomes \\
\hline
\end{tabular}

Ikonomidis 2018

\section{Study characteristics}

Methods
Design: (acute phase) Randomized cross-over assignment (outcomes measured within hours of the intervention and hence do not meet the criteria of 1 week or more); chronic phase: non-randomized, single-group assignment

Recruitment: Hospital smoking cessation unit

Setting: Hospital smoking-cessation unit, Greece

Study start date: 31 January 2017; Study end date: Estimated completion date: December 2021 
Ikonomidis 2018 (Continued)

Inclusion based on specific population characteristic: No

54\% women; mean age 50.2; mean cpd 23.4; mean FTND: Not reported

Motivated to quit: Yes - recruited from smoking cessation unit

E-cigarette use at baseline: Not reported

Interventions

\section{EC: not clear}

E cigarette details: In the chronic phase, all 70 participants were instructed to replace their conventional cigarettes (con-cig) with an e-cig containing nicotine $(12 \mathrm{mg} / \mathrm{dL}$ (e-cig fluid with nicotine concentration of $12 \mathrm{mg} / \mathrm{mL}$ (propylene glycol $74.3 \%$, glycerin 20\%, flavoring $4.5 \%$, nicotine $1.2 \%)$ )) for 1 month

Outcomes

1 month; Self-report and objective measures

Cessation: Self-report cessation at 1 month. CO measured at 1 month. Cessation data not used as $<6$ months

Adverse events and biomarkers:

- Exhaled CO concentration

- Heart rate; blood pressure

Other outcomes measured:

- Oxidative stress as assessed by malondialdehyde (MDA) plasma concentrations

- Aortic stiffness as assessed by pulse wave velocity (PWV) and augmentation index (AIX75)

Study funding This study was supported by a grant from the Hellenic Cardiology Society and Hellenic Society of Lipidiology and Atherosclerosis.

\begin{tabular}{ll}
\hline Author declarations & None \\
\hline Notes & New for 2020 update. Acute phase of trial not relevant for the review as very short-term outcomes
\end{tabular}

\section{Risk of bias}

\begin{tabular}{lll}
\hline Bias & Authors' judgement & Support for judgement \\
\hline $\begin{array}{l}\text { Random sequence genera- } \\
\text { tion (selection bias) }\end{array}$ & Unclear risk & Not specified \\
\hline $\begin{array}{l}\text { Allocation concealment } \\
\text { (selection bias) }\end{array}$ & Unclear risk & Not specified \\
\hline $\begin{array}{l}\text { Blinding of participants } \\
\text { and personnel (perfor- } \\
\text { mance bias) }\end{array}$ & High risk & Not blinded and differential levels of support given \\
All outcomes & \\
\hline
\end{tabular}

Blinding of outcome as-
sessment (detection bias) $\quad$ Unclear risk $\quad$ Objective measures used for all outcomes reported

All outcomes

Incomplete outcome data Low risk 70 participants and 20 controls recruited - no dropout
(attrition bias)
All outcomes

\begin{tabular}{|c|c|c|}
\hline $\begin{array}{l}\text { Selective reporting (re- } \\
\text { porting bias) }\end{array}$ & Unclear risk & $\begin{array}{l}\text { NCT record states that chronic endothelial integrity, platelet aggregation and } \\
\text { high-shear stress-dependent platelet function would be assessed but is not re- }\end{array}$ \\
\hline
\end{tabular}


Ikonomidis 2018 (Continued)

ported in this research letter - however study estimated completion date is December 2021, so perhaps data not ready for publication or limited capacity in the research letter - not the primary publication

Few details - written as commentary. Trial registration suggests this is an ongoing study

Ikonomidis 2020

\section{Study characteristics}

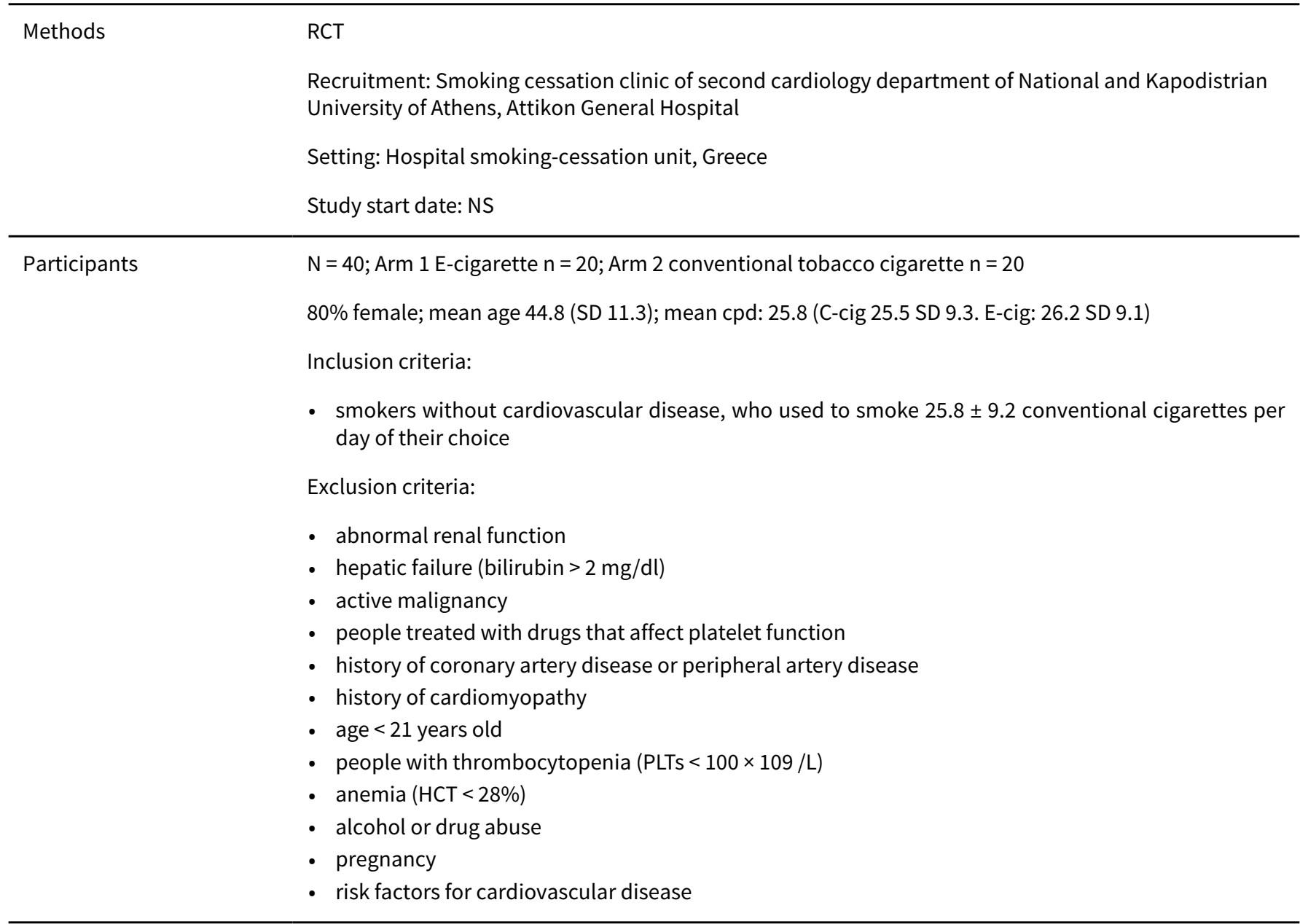

\section{Interventions $\quad$ EC: Refillable}

E-cig: second-generation e-cig device and popular in Greek Market e-liquid (NOBACCO eGo Epsilon BDC 1100 , eGo battery, $1100 \mathrm{mAh}$, operating at $3.9 \mathrm{~V}$ - propylene glycol $74.3 \%$, glycerin $20 \%$, flavoring $4.5 \%$, nicotine $1.2 \% / 12 \mathrm{mg} / \mathrm{ml}$ )

\section{Outcomes Baseline, 4 months: Exhaled CO concentration; blood pressure}

Also, cpd; Ppatelet function by Platelet Function Analyzer PFA-100 and Light Transmission Aggregometry; Pulse wave velocity; Plasma malondialdehyde levels as oxidative stress index

Study funding "There was no funding for this study"


Ikonomidis 2020 (Continued)

Author declarations $\quad$ "The authors declare that they have no known competing financial interests or personal relationships that could have appeared to influence the work reported in this paper."

Notes New for 2021 update

\section{Risk of bias}

Bias Authors' judgement Support for judgement

Random sequence genera- Low risk Table of random numbers as reproduced from the online randomization softtion (selection bias) ware www.graphpad.com/quickcalcs/index

\begin{tabular}{lll}
\hline $\begin{array}{l}\text { Allocation concealment } \\
\text { (selection bias) }\end{array}$ & Unclear risk & Not specified \\
\hline $\begin{array}{l}\text { Blinding of participants } \\
\text { and personnel (perfor- } \\
\text { mance bias) }\end{array}$ & Unclear risk & Not possible \\
All outcomes & \\
\hline
\end{tabular}

\begin{tabular}{lll}
\hline $\begin{array}{l}\text { Blinding of outcome as- } \\
\text { sessment (detection bias) } \\
\text { All outcomes }\end{array}$ & Low risk & Only CO outcomes used here, which are objectively measured \\
\hline $\begin{array}{l}\text { Incomplete outcome data } \\
\begin{array}{l}\text { (attrition bias) } \\
\text { All outcomes }\end{array}\end{array}$ & Low risk & All followed up (confirmed via contact with authors) \\
\hline $\begin{array}{l}\text { Selective reporting (re- } \\
\text { porting bias) }\end{array}$ & Unclear risk & $\begin{array}{l}\text { No protocol or clinical trial record available to confirm whether all prespeci- } \\
\text { fied criteria were reported }\end{array}$ \\
\hline
\end{tabular}

loakeimidis 2018

\section{Study characteristics}

\begin{tabular}{ll}
\hline Methods & Design: Randomized controlled trial \\
Recruitment: Not specified \\
Setting: Hospital, Greece. \\
Study start date/Study end date: Not specified \\
\hline Total N: 54 \\
N per arm: Arm 1: 27; Arm 2: 27 \\
Inclusion criteria: \\
- $\geq 10$ cpd \\
- Motivation to quit \\
- Hospitalized with acute coronary syndrome (ACS) \\
Exclusion criteria: \\
- Prior EC use
\end{tabular}


loakeimidis 2018 (Continued)

- History of neuropsychiatric disorders

- Prior varenicline use or use of SC pharmacotherapy at time of ACS

- Cardiogencic shock or renal impairment

- Hepatic impairment prior to ACS

- Excessive alcohol use or current use of marijuana or non-cigarette tobacco products

Inclusion based on specific population characteristic: People who have experienced acute coronary syndrome

65\% women; mean age 52; mean cpd 21; mean FTND 5.6

Motivated to quit: Yes

E-cigarette use at baseline: No prior EC use

\begin{tabular}{l} 
EC: information on whether cig-a-like or refillable not provided \\
Both arms given "low intensity counselling" \\
Intervention 1: 12 -week use of EC $12 \mathrm{mg} / \mathrm{ml}$ nicotine \\
Intervention 2: 12-week varenicline \\
\hline Weeks: 4, 12, 24 \\
Cessation: 7-day PP at 24 weeks, self-report \\
Adverse events and biomarkers: Unclear how these were reported. Abstract says no SAEs, poster im- \\
plies this may have just been CV or neuropsychiatric SAEs. Abstract says nothing about AEs but nausea \\
and sleeping disorders given in table in poster. Implies (S)AEs collected during treatment period only \\
Other outcomes measured: Not specified
\end{tabular}

\begin{tabular}{ll}
\hline Study funding & Not reported \\
\hline Author declarations & Not reported \\
\hline Notes & New for 2020 update. Abstract and poster only; limited data available \\
\hline
\end{tabular}

\section{Risk of bias}

\begin{tabular}{lll}
\hline Bias & Authors' judgement & Support for judgement \\
\hline $\begin{array}{l}\text { Random sequence genera- } \\
\text { tion (selection bias) }\end{array}$ & Unclear risk & Not specified \\
\hline $\begin{array}{l}\text { Allocation concealment } \\
\text { (selection bias) }\end{array}$ & Unclear risk & Not specified \\
\hline $\begin{array}{l}\text { Blinding of participants } \\
\text { and personnel (perfor- } \\
\text { mance bias) } \\
\text { All outcomes }\end{array}$ & Low risk & $\begin{array}{l}\text { Not specified but equal amounts of contact and support between arms so per- } \\
\text { formance bias judged unlikely }\end{array}$ \\
\hline $\begin{array}{l}\text { Blinding of outcome as- } \\
\text { sessment (detection bias) } \\
\text { All outcomes }\end{array}$ & Low risk & \\
\hline $\begin{array}{l}\text { Incomplete outcome data } \\
\text { (attrition bias) }\end{array}$ & Unclear risk & $\begin{array}{l}\text { Self-report only but equal amounts of contact between arms, no reason to sus- } \\
\text { pect differential misreport }\end{array}$ \\
\hline
\end{tabular}


Ioakeimidis 2018 (Continued)

All outcomes

Selective reporting (re- Unclear risk $\quad$ Abstract/poster only so not able to judge
porting bias)

Other bias High risk

Abstract and poster only. Two different figures presented for quit rate in EC arm (no difference in those presented in varenicline arm) between abstract and poster. Poster percentage aligns with figure, so using that (16.5\%) as opposed to abstract figure (32.5\%). Contacted authors but no reply. Calculated $n$ quit based on percentages but unclear what denominators were; EC calculates back to whole number for EC but not for varenicline

\section{Study characteristics}

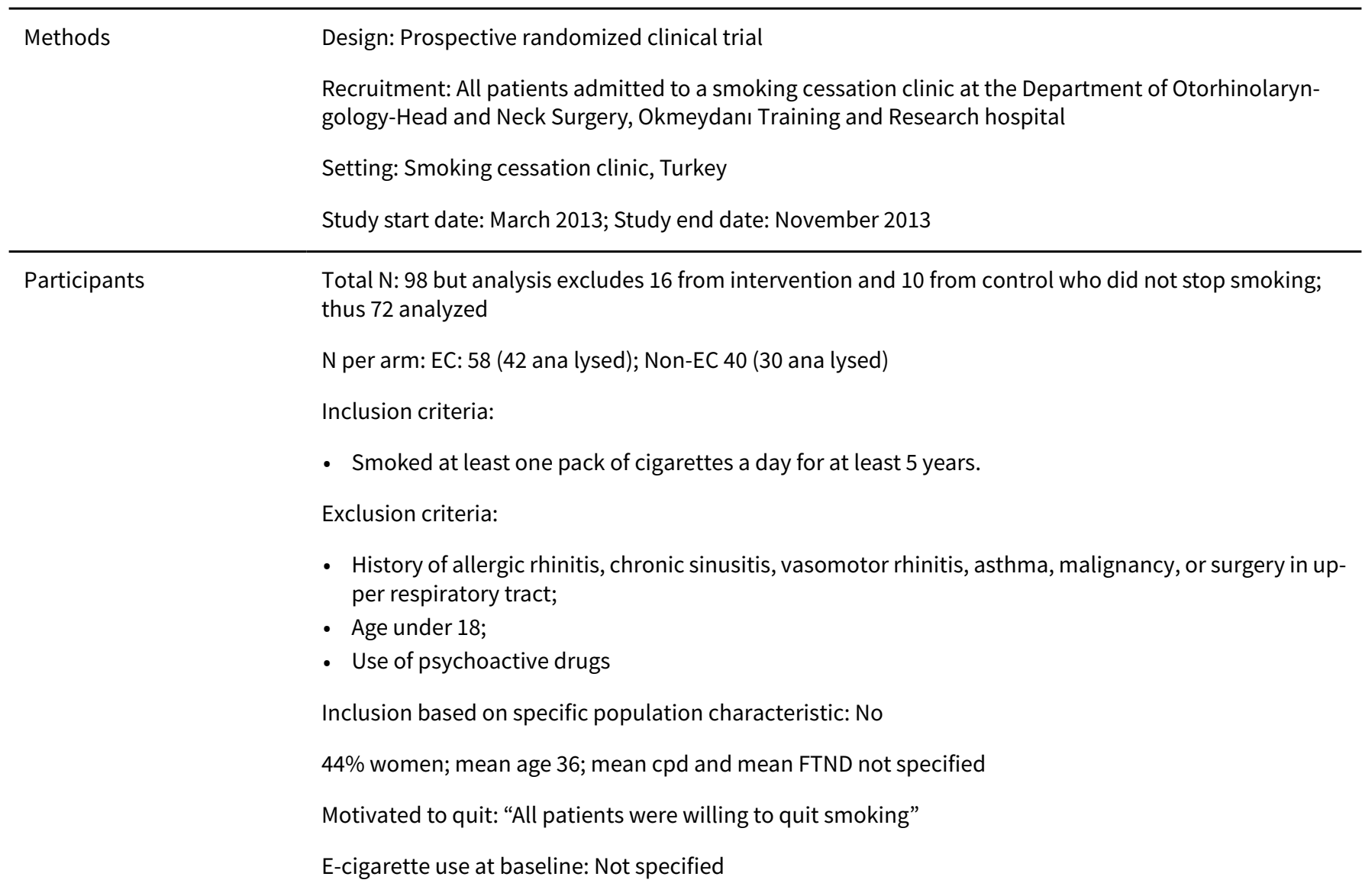

Interventions

\section{EC: Unclear}

EC arm: "used EC to quit smoking" - allowed to select brand and flavor, used "medium density" liquid (11 $-12 \mathrm{mg} / \mathrm{ml}$ ) (no further detail given)

Non-EC arm: Received cognitive behavioral therapy (no further detail given)

Outcomes 3 Months


Kumral 2016 (Continued)

Sino-nasal outcome test (SNOT-22) via self-administered questionnaire, to evaluate changes in subjective symptoms. Saccharin transit test to evaluate nasal mucociliary clearance (MCC) function which authors state is "an important defence mechanism"

\begin{tabular}{ll}
\hline Study funding & Not specified \\
\hline Author declarations & Not specified \\
\hline Notes &
\end{tabular}

\section{Risk of bias}

Bias Authors' judgement Support for judgement

Random sequence genera- Unclear risk tion (selection bias)
Quote: "Patients participating in the study were randomly divided into two groups; EC smokers (group 1) and non-EC smokers (group 2).”

No further detail provided

\begin{tabular}{|c|c|c|}
\hline $\begin{array}{l}\text { Allocation concealment } \\
\text { (selection bias) }\end{array}$ & Unclear risk & No information given \\
\hline $\begin{array}{l}\text { Blinding of participants } \\
\text { and personnel (perfor- } \\
\text { mance bias) } \\
\text { All outcomes }\end{array}$ & High risk & $\begin{array}{l}\text { Participants were not blinded. The trial is described as single-blinded and out- } \\
\text { come assessors were blinded. No placebo used }\end{array}$ \\
\hline $\begin{array}{l}\text { Blinding of outcome as- } \\
\text { sessment (detection bias) } \\
\text { All outcomes }\end{array}$ & High risk & $\begin{array}{l}\text { Self-reported outcome data, participants not blinded and unequal amounts of } \\
\text { support between arms }\end{array}$ \\
\hline $\begin{array}{l}\text { Incomplete outcome data } \\
\text { (attrition bias) } \\
\text { All outcomes }\end{array}$ & Unclear risk & Dropout rate not clear. Only ana lysed people who quit \\
\hline $\begin{array}{l}\text { Selective reporting (re- } \\
\text { porting bias) }\end{array}$ & Low risk & All expected outcomes reported \\
\hline
\end{tabular}

Lee 2018

\section{Study characteristics}

\begin{tabular}{ll}
\hline Methods & Randomized parallel-assignment double-blind pilot trial \\
& Setting: San Francisco Veterans Affairs Medical Center (SFVAMC), USA \\
& Recruitment: veterans awaiting surgery \\
& Recruitment: In VA hospital presenting for surgery \\
& Study start date: August 2015; Study end date: May 2016 \\
Participants & Total N: 50 \\
N per arm: NRT: $30 ;$ END: 20 \\
Inclusion criteria:
\end{tabular}


Lee 2018 (Continued)

- Presented to the anesthesia preoperative clinic for elective surgery 3 or more days before surgery

- Currently smoked more than 2 cigarettes per day, having smoked at least once in the last 7 days

Exclusion criteria:

- Exclusively used other forms of tobacco or marijuana only

- Pregnant or breastfeeding

- Unstable cardiac condition

- Currently using smoking cessation pharmacotherapy

- Were already enrolled in a smoking cessation trial

- Currently used e-cigarettes on a daily basis

Inclusion based on specific population characteristic: Patients awaiting elective surgery

$10 \%$ women; mean age 54; mean cpd 14; mean FTND 3.3

Motivated to quit: Not specified

E-cigarette use at baseline: Not specified but excluded daily users

Interventions

\section{EC: Cig-a-like}

Both groups receive: i) referral to the California Smokers' Helpline, ii) brief advice lasting less than 2 minutes, iii) a brochure from the ASA about quitting smoking before surgery

EC arm: 6-week supply of NJOY e-cigarettes (disposable, first generation). Instructed to use Bold (4.5\%) ad lib for 3 weeks, then Gold (2.4\%) ad lib for 2 weeks and then study (0\%) ad lib for final week. Number of ECs issued corresponded to baseline cpd, assuming $1 \mathrm{EC}=10$ cigarettes. Asked to refrain from the use of all study products at the end of 6 weeks

NRT arm: 5-week Nicoderm CQ patches, 1 week placebo patches. Dose based on cpd at baseline: $\geq 10$ cpd, $21 \mathrm{mg} /$ day for 3 weeks, $14 \mathrm{mg} /$ day for 1 week, $7 \mathrm{mg} /$ day for 1 week, $0 \mathrm{mg} /$ day for 1 week. $<10 \mathrm{cpd}$ at baseline: $14 \mathrm{mg} /$ day for 3 weeks, $7 \mathrm{mg} /$ day for 2 weeks, $0 \mathrm{mg} /$ day for 1 week

Outcomes

30 Days (phone), 8 Weeks (in person), 6 Months (phone)

Cessation: 7-day PP at 30 days (not validated), 8 weeks (CO-validated), 6 months (not validated). Smoking cessation for at least 48 hours on day of surgery (CO-validated)

Adverse events and biomarkers:

- Adverse events, side effects, and surgical complications by self-report at 30 days, 8 weeks

- At 8 weeks exhaled CO, FEV1 and FVC

Other outcomes measured:

- Attitudes and usage

- Salivary cotinine

- Smoking reduction

Study funding

"This work was funded by internal UCSF Department of Anesthesia and Perioperative Care funds (San Francisco, California, United States of America) and the UCSF Resource Allocation Program grant, administered by the Helen Diller Family Comprehensive Cancer Center developmental funds from the National Cancer Institute Cancer Center Support Grant (P30 CA 82103-16). E-cigarettes were purchased from NJOY using these funds. NJOY had no involvement in the design, execution, or analysis of the study. The funders had no role in study design, data collection and analysis, decision to publish, or preparation of the manuscript."

\begin{tabular}{ll}
\hline Author declarations & "The authors declare there are no competing interests" \\
\hline Notes & 3 NRT participants used EC, 2 EC participants used nicotine patch
\end{tabular}


Lee 2018 (Continued)

Study listed as ongoing study NCT02482233 in the 2016 review update

\section{Risk of bias}

\begin{tabular}{|c|c|c|}
\hline Bias & Authors' judgement & Support for judgement \\
\hline $\begin{array}{l}\text { Random sequence genera- } \\
\text { tion (selection bias) }\end{array}$ & Low risk & $\begin{array}{l}\text { Quote: "Randomization was computer-generated, with randomly permuted } \\
\text { block sizes of } 3 \text { or } 6 \text {, in a 2:1 ratio using the ralloc program" }\end{array}$ \\
\hline $\begin{array}{l}\text { Allocation concealment } \\
\text { (selection bias) }\end{array}$ & Low risk & $\begin{array}{l}\text { Quote: "Allocation was concealed by consecutively numbered, sealed, opaque } \\
\text { envelopes" }\end{array}$ \\
\hline $\begin{array}{l}\text { Blinding of participants } \\
\text { and personnel (perfor- } \\
\text { mance bias) } \\
\text { All outcomes }\end{array}$ & Low risk & $\begin{array}{l}\text { Not blinded but both interventions active with equal amounts of support so } \\
\text { performance bias judged unlikely }\end{array}$ \\
\hline $\begin{array}{l}\text { Blinding of outcome as- } \\
\text { sessment (detection bias) } \\
\text { All outcomes }\end{array}$ & Low risk & $\begin{array}{l}\text { Self-report only at } 6 \text { months and participants not blinded to condition, but } \\
\text { similar level of support given to both groups so differential misreport judged } \\
\text { unlikely }\end{array}$ \\
\hline $\begin{array}{l}\text { Incomplete outcome data } \\
\text { (attrition bias) } \\
\text { All outcomes }\end{array}$ & Low risk & $1 \mathrm{NRT}$ and 1 ENDs loss to follow-up at 6 months \\
\hline $\begin{array}{l}\text { Selective reporting (re- } \\
\text { porting bias) }\end{array}$ & Low risk & All expected outcomes reported \\
\hline
\end{tabular}

Lee 2019

\section{Study characteristics}

\begin{tabular}{ll} 
Methods & Design: Randomized controlled trial \\
Recruitment: Recruited from motor company. \\
Setting: Motor company, medical office in Korea \\
Study start date: 5 January 2012; Study end date: 31 August 2012 \\
\hline Total N: 150 \\
N per arm: EC: $75 ;$ NRT: 75 \\
Inclusion criteria: \\
- Male \\
- At least 10 cpd in previous year \\
- Smoked for at least 3 years \\
- Motivate to stop smoking entirely or reduce consumption \\
Exclusion criteria: \\
- Past history of serious clinical disease \\
- Attempted to stop smoking in past 12 months by using NRTs \\
Inclusion based on specific population characteristic: No
\end{tabular}


Lee 2019 (Continued)

0\% women; mean age 42.3; mean cpd: Not reported, 1.01 packs per day; mean FTND 4.05

Motivated to quit: Yes, or to reduce

E-cigarette use at baseline: Not specified

Interventions

\section{EC: Refillable}

Both arms received 50 mins education session on smoking cessation and use of smoking cessation aids in medical office (no further detail given). Asked to return to medical office every 4 weeks (to 24 weeks?) for "evaluation and counseling by an independent health practitioner"

Arm 1: 50-min education sessions on smoking cessation and the use of smoking-cessation aids, instructed to visit the medical office each month for evaluation and counseling by a health practitioner who was unaffiliated with the study. Participants supplied with eGo-CTM EC (nicotine $0.01 \mathrm{mg} / \mathrm{mL}$ ) from Ovale in 12-wk supply

Arm 2: As (1) but instead of EC given 2 mg nicotine gum in 12-wk supply

Outcomes $\quad 12,24$ weeks (in person)

Cessation: continuous abstinence from 9 - 24 weeks, exhaled $\mathrm{CO}<10 \mathrm{ppm}$, negative urine cotinine Adverse events and biomarkers: Yes but just note 'adverse events'

Other outcomes measured: 7-day PPA, cigarette reduction

\begin{tabular}{ll}
\hline Study funding & "none" \\
\hline Author declarations & "none declared" \\
\hline Notes & Study listed as ongoing study KCT0001277 in the 2016 review update
\end{tabular}

\section{Risk of bias}

\begin{tabular}{|c|c|c|}
\hline Bias & Authors' judgement & Support for judgement \\
\hline $\begin{array}{l}\text { Random sequence genera- } \\
\text { tion (selection bias) }\end{array}$ & Low risk & Quote: "computer-generated randomization sequence with a block size of 2" \\
\hline $\begin{array}{l}\text { Allocation concealment } \\
\text { (selection bias) }\end{array}$ & Low risk & $\begin{array}{l}\text { Quote: "The enrolment and assignment of all subjects were performed by a } \\
\text { clinical research coordinator not involved in the study" }\end{array}$ \\
\hline $\begin{array}{l}\text { Blinding of participants } \\
\text { and personnel (perfor- } \\
\text { mance bias) } \\
\text { All outcomes }\end{array}$ & Low risk & $\begin{array}{l}\text { Not blinded but both interventions active with equal amounts of support, so } \\
\text { performance bias judged unlikely }\end{array}$ \\
\hline $\begin{array}{l}\text { Blinding of outcome as- } \\
\text { sessment (detection bias) } \\
\text { All outcomes }\end{array}$ & Low risk & Participants not blinded but results biochemically validated \\
\hline $\begin{array}{l}\text { Incomplete outcome data } \\
\text { (attrition bias) } \\
\text { All outcomes }\end{array}$ & Low risk & $61 / 75$ NRT and $71 / 75$ EC FU at 24 weeks \\
\hline $\begin{array}{l}\text { Selective reporting (re- } \\
\text { porting bias) }\end{array}$ & Low risk & All prespecified outcomes reported \\
\hline
\end{tabular}


Lucchiari 2020

\section{Study characteristics}

Methods
Design: Randomized parallel-assignment double-blind trial

Recruitment: Participants enrolled in lung cancer-screening program

Setting: Early lung cancer detection program (Cosmos II) at European Institute of Oncology, Italy

Study start date: September 2014; Study end date: January 2016
Total N: 210

N per arm: 70 participants per arm

Inclusion criteria:

- Participants are involved in the cosmos II study

- Participants are 55 years or more and have smoked at least 10 cigarettes a day for the past 10 years

- Participants wish to reduce tobacco smoking (motivational score higher than 10) who are not treated at a smoking centre

- Signed informed consent

Exclusion criteria:

- Symptomatic cardiovascular disease

- Symptomatic severe respiratory disease

- Regular psychotropic medication use

- Current or past history of alcohol abuse

- Use of smokeless tobacco or NRT

- Participation in another antismoking program in the current year

Inclusion based on specific population characteristic: 55 years of age or older

$37 \%$ women; mean age 62.8; mean cpd 19.38; mean FTND 4.37

Motivated to quit: yes

E-cigarette use at baseline: Excluded people who smoke who had ever regularly used e-cigarettes for more than 1 week alone or in combination with tobacco cigarettes

\section{EC: Cig-a-like}

Both arms received "low intensity counseling" - phone at week 1, 4, 8 and 12, approx. 10 mins each

Nicotine EC arm: e-cigarette kit and 12 10-mL liquid cartridges ( $8 \mathrm{mg} / \mathrm{mL}$ nicotine concentration). During the first week, participants could use the e-cigarette ad libitum. At the end of the first week, asked to use only EC for the next 11 weeks

Nicotine-free EC (placebo) arm: Nicotine-free EC - same as above but with nicotine-free EC
Months 3, 6 and 12 (but only 3- and 6-month data available)

Cessation: Continuous abstinence for previous month, $\mathrm{CO} \leq 7 \mathrm{ppm}$

Adverse events and biomarkers: FOR EC ARMS ONLY:

- Exhaled CO

- Leicester Cough Questionnaire (LCQ)

- Respiratory symptoms (self-report)

- Side effects using checklist 
Lucchiari 2020 (Continued)

Other outcomes measured:

- Motivational questionnaire

- HADS

- EC use

\begin{tabular}{ll}
\hline Study funding & This study was supported by a grant from Fondazione Umberto Veronesi (FUV) \\
\hline Author declarations & The authors declare no conflicts of interest \\
\hline Notes & Listed as ongoing study Lucchiari 2016 (NCT02422914) in 2016 review; new for 2020 update \\
\hline
\end{tabular}

\section{Risk of bias}

\begin{tabular}{|c|c|c|}
\hline Bias & Authors' judgement & Support for judgement \\
\hline $\begin{array}{l}\text { Random sequence genera- } \\
\text { tion (selection bias) }\end{array}$ & Low risk & $\begin{array}{l}\text { Quote: "A randomization list using a permuted block design ( } 40 \text { blocks of } 6 \\
\text { subjects randomly assigned to } 1 \text { of the } 3 \text { treatment arms) had been previously } \\
\text { prepared by independent personnel." }\end{array}$ \\
\hline $\begin{array}{l}\text { Allocation concealment } \\
\text { (selection bias) }\end{array}$ & Low risk & $\begin{array}{l}\text { Double-blind, active and placebo e-cigarettes labeled by independent person- } \\
\text { nel, researcher and participants blind }\end{array}$ \\
\hline $\begin{array}{l}\text { Blinding of participants } \\
\text { and personnel (perfor- } \\
\text { mance bias) } \\
\text { All outcomes }\end{array}$ & Low risk & $\begin{array}{l}\text { "double blind" for nicotine vs no nicotine EC but limited info given; however, } \\
\text { as similar levels of support across arms performance bias judged unlikely }\end{array}$ \\
\hline $\begin{array}{l}\text { Blinding of outcome as- } \\
\text { sessment (detection bias) } \\
\text { All outcomes }\end{array}$ & Low risk & Biochemical validation used \\
\hline $\begin{array}{l}\text { Incomplete outcome data } \\
\text { (attrition bias) } \\
\text { All outcomes }\end{array}$ & Low risk & $\begin{array}{l}\text { Approx. } 73 \% \text { followed up in each group at } 6 \text { months, very little difference be- } \\
\text { tween groups }\end{array}$ \\
\hline $\begin{array}{l}\text { Selective reporting (re- } \\
\text { porting bias) }\end{array}$ & High risk & $\begin{array}{l}\text { Paper states data also collected at } 12 \mathrm{~m} \text { but this is not presented and unclear } \\
\text { why. Paper states } \mathrm{CO} \text { collected but data not presented }\end{array}$ \\
\hline
\end{tabular}

\section{Martner 2019}

\section{Study characteristics}

Design: A nonconcurrent multiple baseline across participants design. Three phases were included:
Baseline, EC, and EC + CM. Half the participants received the EC phase following baseline; the other half
received EC + CM following baseline
Recruitment: Community
Setting: Set-up meetings occurred at the University of Florida Behavioral Health and Technology Re-
search Clinic, USA
Study start date/Study end date: Not specified.
Potal N: 12
Inclusion criteria:


Martner 2019 (Continued)

- 18 - 65 years old

- Smoked $\geq 2$ years

- Smoked $\geq 8 \mathrm{cpd}$ on average

- Smoked in the past 24 hours

- Expressed a desire to quit smoking (yes/no)

- Had reliable access to the internet and a computer or smartphone

- Breath $C O \geq 10$ ppm at set-up

Exclusion criteria:

- Current or previous medical condition that would pose an increased risk to participation

- Use of benzodiazepines, cocaine, or opiates in the previous 6 months

- Smoke marijuana more than twice a month

- Exposed to elevated CO levels (e.g. spouse smokes in house)

- Pregnant or expected to become pregnant in the next 6 months

58.3\% women; mean age 37.5; mean cpd 16.25; mean FTND 5

Motivated to quit: Expressed a desire to quit smoking.

E-cigarette use at baseline: 3 participants never tried an EC prior to the study; 2 owned an EC but quit using it more than a month prior to the study; remaining 7 had tried an EC more than a year prior to the study but never owned one

Interventions

\section{EC: Refillable}

All participants provided with smokio electronic cigarettes (second-generation ECs) and V2 e-liquid with a concentration of $24 \mathrm{mg} / \mathrm{ml}(2.4 \%)$ of nicotine. Researchers provided participants with a copy of the National Cancer Institute's brochure Clearing the Air (http://smokefree. gov). Then researchers and participants read through a manual that described the study procedures, and showed participants how to use the software to measure $\mathrm{CO}$ and how to use the EC

Participants initially received EC without contingency for a period of 14 days following the quit attempt. If participants failed to reduce $\mathrm{CO}$ levels during this phase, they received contingency management in addition to EC

\begin{tabular}{|c|c|c|}
\hline \multirow[t]{3}{*}{ Outcomes } & \multicolumn{2}{|l|}{4 weeks } \\
\hline & \multicolumn{2}{|c|}{$\begin{array}{l}\text { Adverse events and biomarkers: Adverse events collected in 4-day smoking behavior questionnaires; } \\
\text { eCO }\end{array}$} \\
\hline & \multicolumn{2}{|c|}{ Other outcomes measured: acceptability and use of EC; overall experience of study } \\
\hline Study funding & \multicolumn{2}{|c|}{$\begin{array}{l}\text { "The study was supported in part by crowd-sourced funding enabled by Experiment.com. Preparation } \\
\text { of this paper was supported in part by Grant P30DA029926." }\end{array}$} \\
\hline Author declarations & \multicolumn{2}{|c|}{ "The authors declare no conflicts of interest." } \\
\hline Notes & \multicolumn{2}{|c|}{$\mathrm{N}$ of 1 (within-participants randomized design, not between groups). New for 2020 update. } \\
\hline \multicolumn{3}{|l|}{ Risk of bias } \\
\hline Bias & Authors' judgement & Support for judgement \\
\hline $\begin{array}{l}\text { Random sequence genera- } \\
\text { tion (selection bias) }\end{array}$ & High risk & Not randomized \\
\hline $\begin{array}{l}\text { Allocation concealment } \\
\text { (selection bias) }\end{array}$ & High risk & Not randomized \\
\hline
\end{tabular}


Martner 2019 (Continued)
Incomplete outcome data
Unclear risk
No details provided.
(attrition bias)

All outcomes

Selective reporting (re- Unclear risk $\quad$ AEs measured in behavioral change questionnaire but not reported
porting bias)

\section{McRobbie 2015}

\section{Study characteristics}

\begin{tabular}{ll} 
Methods & Design: Prospective cohort \\
Recruitment: advertisements in free London newspapers \\
Setting: Smokers' clinic, East London, UK \\
Study start date: February 2013; Study end date: September 2013 \\
\hline Total N: 40 \\
Inclusion criteria: \\
Participants \\
- People who smoke daily who want to quit 18 and older \\
Exclusion criteria: \\
- Pregnant and breastfeeding women \\
- Current serious medical illness \\
- EC use for more than 1 week in the past \\
45\% women, mean age 47 (SD 12), mean cpd 19 (SD 10), mean FTND 5.2 (SD 2.8), 65\% in full-time em- \\
ployment \\
Motivated to quit: Yes \\
E-cigarette use at baseline: Excluded those who had used EC for more than 1 week in the past
\end{tabular}

Interventions

\section{EC: Cig-a-like}

Participants attended baseline session 1 week prior to their TQD. On the TQD, participants were provided with an EC (Green Smoke, 1st generation device, 2.4\% nicotine cartridges). 2 cartridges a day were supplied initially, with the supply adjusted to actual use later. Attended 4 weekly follow-up sessions and received standard behavioral support

Outcomes Cigarette consumption and $\mathrm{CO}$ readings collected at each session. Urine sample for cotinine and 3-HPMA analysis collected at baseline and 4 weeks post-TQD

Change in urinary 3-HPMA ( $\mathrm{ng} / \mathrm{mg}$ creatinine) at 4 weeks

Change in urinary cotinine ( $\mathrm{ng} / \mathrm{mg}$ creatinine) at 4 weeks

Change in $\mathrm{CO}$ at 4 weeks

Study funding

"This study was funded by a grant given to P. Hajek, H. McRobbie, and M.L.Goniewicz from the UK Medicines and Healthcare Products Regulatory Agency (MHRA). The costs of publication of this article were defrayed in part by the payment of page charges. This article must therefore be hereby marked advertisement in accordance with 18 U.S.C. Section 1734 solely to indicate this fact." 
McRobbie 2015 (Continued)

Author declarations
"H. McRobbie is Clincal Director at The Dragon Institute; reports receiving commercial research grant from Pfizer; and has received speakers bureau honoraria from Johnson\&Johnson and Pfizer. M.L. Goniewicz reports receiving commercial research grant from Pfizer. P. Hajek has received speakers bureau honoraria from and is a consultant/advisory board member for the manufacturers of stop-smoking medications. No potential conflicts of interest were disclosed by the other authors."

Notes

\section{Risk of bias}

Bias Authors' judgement Support for judgement

Random sequence genera- High risk Prospective cohort

tion (selection bias)

\begin{tabular}{lll}
\hline $\begin{array}{l}\text { Allocation concealment } \\
\text { (selection bias) }\end{array}$ & High risk & Not randomized \\
\hline $\begin{array}{l}\text { Incomplete outcome data } \\
\text { (attrition bias) }\end{array}$ & Low risk & $7 / 40$ participants were lost to follow-up \\
All outcomes & & \\
\hline
\end{tabular}

Selective reporting (re- Low risk All predefined outcomes reported

porting bias)

Meier 2017

\section{Study characteristics}

Methods

Design: Randomized cross-over trial (e-cig vs placebo)

Recruitment: via local media outlets

Setting: Community, USA

Study start date/Study end date: Not specified.

Participants
Inclusion criteria:
- $\geq 18$,
- People who smoke daily $(\geq 10 \mathrm{cpd})$
- Not interested in quitting in next 30 days
- English-speaking
- Interested in using EC
Exclusion criteria:
- Using cessation meds
- Use of ECs in last $6 \mathrm{~m}$
- Exhaled CO $<6 \mathrm{ppm}$,
- History of CV trauma or uncontrolled hypertension
- Pregnant

Inclusion based on specific population characteristic: No 
Meier 2017 (Continued)

25\% women; mean age 48.5; mean cpd 16.3; FTND not reported

Motivated to quit: No (eligibility criteria was to not want to quit in next 30 days)

E-cigarette use at baseline: $8 / 24$ (33\%) had previously tried an EC, avg 9.4 months since last use, avg length of use 3.6 days

Interventions

\section{EC: Cig-a-like}

Smoked "as usual" for 1 week followed by 2 weeks of either placebo or active 1st generation EC BluCig starter kit with up to 7 cartridges (prefilled, with either active $16 \mathrm{mg}$ or $0 \mathrm{mg}$ nicotine solution)

Participants were instructed "this e-cig may or may not contain nicotine; we ask that you try it at least once, but use it however you like; smoke regular cigarettes as you wish." Shown how to charge the device and sampled the product during the visit. Provided a handout on how to use the product (e.g., switching cartridges) and general information about ECs

\begin{tabular}{|c|c|}
\hline Outcomes & $\begin{array}{l}1 \text { week in each condition, in person } \\
\text { Adverse events and biomarkers: } \\
\text { - Adverse events, not clear how collected } \\
\text { - Exhaled CO } \\
\text { Other outcomes measured: } \\
\text { - Vaping } \\
\text { - Regular smoking } \\
\text { - Perceived reward from ECs } \\
\text { - Intentions/confidence to quit } \\
\text { - Cotinine } \\
\text { - Withdrawal symptoms }\end{array}$ \\
\hline
\end{tabular}

Study funding

“..supported by grants P01 CA138389, P30 CA138313 (Hollings Cancer Center Support Grant) from the National Cancer Institute of the National Institutes of Health and UL1 TR000062 from the National Center for Advancing Translational Science of the National Institutes of Health. BWH was supported by K12DA031794"

Author declarations

"KMC has received grant funding from the Pfizer, Inc., to study the impact of a hospital-based tobacco cessation intervention. He also receives funding as an expert witness in litigation filed against the tobacco industry. We have no other declarations of interests to declare"

Notes New for 2020 update.

\section{Risk of bias}

\begin{tabular}{lll}
\hline Bias & Authors' judgement & Support for judgement \\
\hline $\begin{array}{l}\text { Random sequence genera- } \\
\text { tion (selection bias) }\end{array}$ & Unclear risk & $\begin{array}{l}\text { Quote: "Participants were randomized to receive either an active or placebo } \\
\text { EC first", no further information provided. }\end{array}$ \\
\hline $\begin{array}{l}\text { Allocation concealment } \\
\text { (selection bias) }\end{array}$ & Unclear risk & Refer to 'Random sequence generation'. \\
\hline $\begin{array}{l}\text { Blinding of participants } \\
\text { and personnel (perfor- } \\
\text { mance bias) }\end{array}$ & Low risk & $\begin{array}{l}\text { Quote: "Participants and research staff conducting sessions were blinded to } \\
\text { dose. All cartridges were pre-loaded by the manufacturer. Labeling was re- } \\
\text { moved by a research team member not involved in participant contact to } \\
\text { mask placebo versus active ECs. We restricted flavor options to regular tobac- } \\
\text { co flavor or menthol to most closely match usual cigarette brand flavor profile } \\
\text { and reduce unwanted variance in product" }\end{array}$ \\
\hline
\end{tabular}


Meier 2017 (Continued)

Blinding of outcome as- Low risk Quote: "Participants and research staff conducting sessions were blinded to sessment (detection bias)

All outcomes dose. All cartridges were pre-loaded by the manufacturer. Labeling was removed by a research team member not involved in participant contact to mask placebo versus active ECs. We restricted flavor options to regular tobacco flavor or menthol to most closely match usual cigarette brand flavor profile and reduce unwanted variance in product"

\begin{tabular}{lll}
$\begin{array}{l}\text { Incomplete outcome data } \\
\text { (attrition bias) } \\
\text { All outcomes }\end{array}$ & Unclear risk & Not specified \\
\hline $\begin{array}{l}\text { Selective reporting (re- } \\
\text { porting bias) }\end{array}$ & Low risk & All expected outcomes reported \\
\hline
\end{tabular}

NCT02648178

\section{Study characteristics}

\begin{tabular}{|c|c|}
\hline \multirow[t]{5}{*}{ Methods } & Setting: Medical centre, USA \\
\hline & Recruitment: People with cancer \\
\hline & Design: Non-randomized single-group assignment trial \\
\hline & Recruitment: Clinical settings, including outpatient clinics and the infusion suite \\
\hline & Study start date: June 2016; Study end date: May 2018 \\
\hline \multirow[t]{17}{*}{ Participants } & Total N: 19 \\
\hline & Inclusion criteria: \\
\hline & $\begin{array}{l}\text { - Histological or cytological diagnosis of aerodigestive tract cancers or bladder cancer within the past } \\
5 \text { years (more than } 1 \text { tobacco-related malignancy is allowed) }\end{array}$ \\
\hline & - AJCC stages I - IV \\
\hline & - Daily smoking (at least 10 cigarettes per day for 10 years) and breath $\mathrm{CO}^{2} \geq 8 \mathrm{ppm}$ \\
\hline & $\begin{array}{l}\text { - Does not wish to quit smoking now (anyone wishing to quit smoking will be referred for smoking ces- } \\
\text { sation counseling through the WRJ VAMC or DHMC program) }\end{array}$ \\
\hline & - May be receiving anti-cancer agents \\
\hline & - Age 18 or older \\
\hline & - Fluent in English \\
\hline & - Patient must be capable and willing to provide informed written consent for study participation \\
\hline & - Able to participate in study visits \\
\hline & Exclusion criteria: \\
\hline & - Cancer surgery planned in the next 9 weeks \\
\hline & - Treatment with radiation planned for the next 9 weeks \\
\hline & $\begin{array}{l}\text { - Actively trying to quit smoking, or planning to in the next } 30 \text { days. (If a patient reports that they plan to } \\
\text { quit smoking in the next } 30 \text { days, we will call them after the } 30 \text { days to see if they are still trying to quit) }\end{array}$ \\
\hline & - Any use of e-cigarettes in the past 30 days \\
\hline & - Pregnant or trying to get pregnant \\
\hline
\end{tabular}

Inclusion based on specific population characteristic: Patients with stage I - IV aerodigestive tract cancers or bladder cancer who smoke daily 
NCT02648178 (Continued)

42.1\% women; mean age: not reported -categories 18 - 65 years: $N=9,>65$ years: $N=10$; cpd and FTND: not reported.

Motivated to quit: No (inclusion criterion)

E-cigarette use at baseline: Not specified but EC use within 30 days is an exclusion criterion

\section{EC: Cig-a-like and refillable}

Instructed on use of EC, and given a supply that is "approximately equivalent to their current nicotine intake". Given Halo Triton EC (leak-proof refillable tank system) or Halo G6 leak proof prefilled cartomizers. Began participants with $18 \mathrm{mg} / \mathrm{ml}$ and moved nicotine content up or down based on participant preference. Choice of flavors, provided for 9 weeks

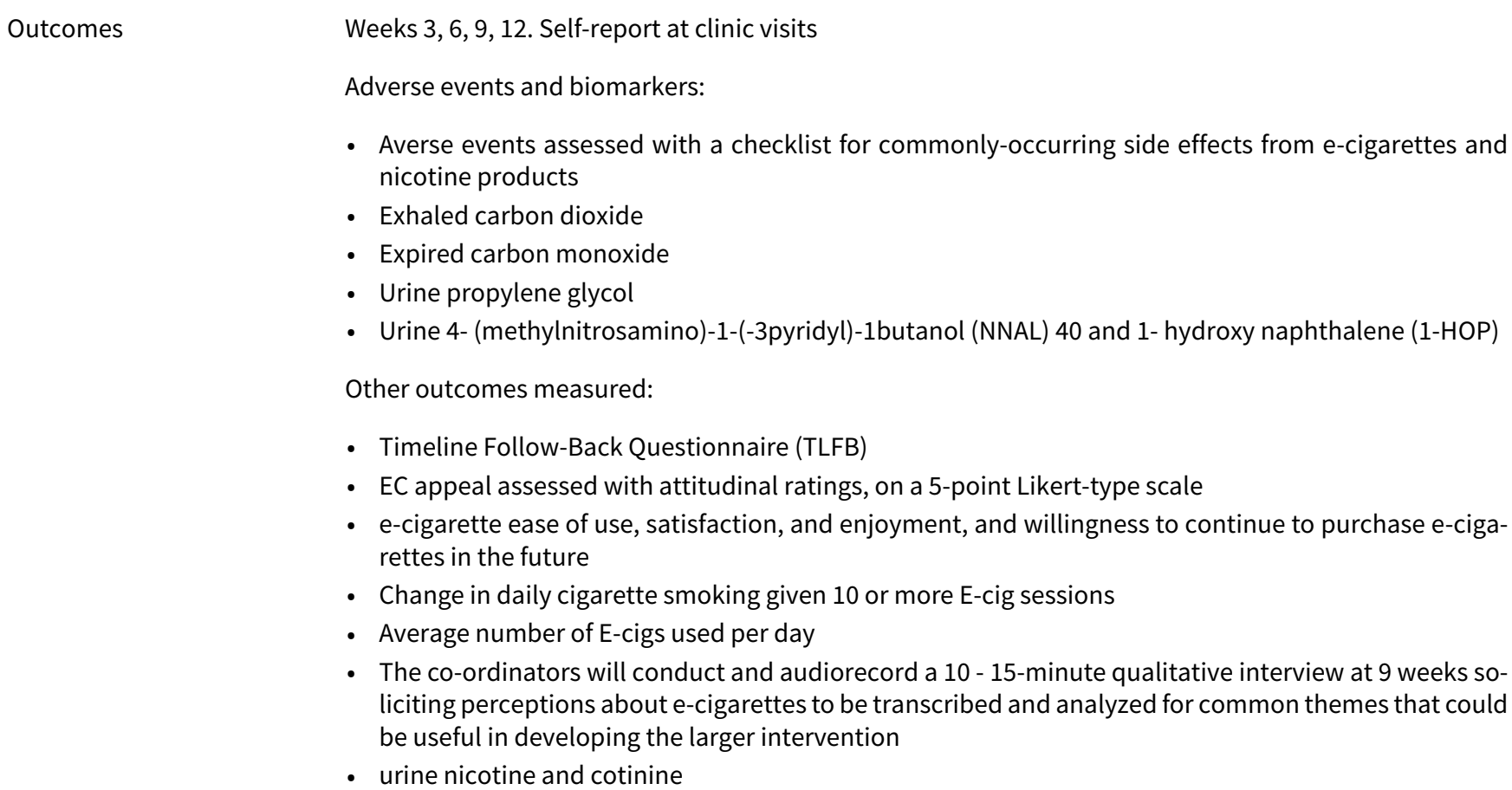

- Averse events assessed with a checklist for commonly-occurring side effects from e-cigarettes and nicotine products

- Exhaled carbon dioxide

- Expired carbon monoxide

- Urine propylene glycol

- Urine 4- (methylnitrosamino)-1-(-3pyridyl)-1butanol (NNAL) 40 and 1- hydroxy naphthalene (1-HOP)

Other outcomes measured:

- Timeline Follow-Back Questionnaire (TLFB)

- EC appeal assessed with attitudinal ratings, on a 5-point Likert-type scale

- e-cigarette ease of use, satisfaction, and enjoyment, and willingness to continue to purchase e-cigarettes in the future

- Change in daily cigarette smoking given 10 or more E-cig sessions

- Average number of E-cigs used per day

- The co-ordinators will conduct and audiorecord a 10 - 15-minute qualitative interview at 9 weeks soliciting perceptions about e-cigarettes to be transcribed and analyzed for common themes that could be useful in developing the larger intervention

- urine nicotine and cotinine

\begin{tabular}{ll}
\hline Study funding & Not reported - data extracted from clinical trial registry record \\
\hline Author declarations & Not reported - data extracted from clinical trial registry record \\
\hline Notes & Study listed as ongoing study in the 2016 review update
\end{tabular}

\section{Risk of bias}

\begin{tabular}{lll}
\hline Bias & Authors' judgement & Support for judgement \\
\hline $\begin{array}{l}\text { Random sequence genera- } \\
\text { tion (selection bias) }\end{array}$ & High risk & Not randomized, single-group assignment \\
\hline $\begin{array}{l}\text { Allocation concealment } \\
\text { (selection bias) }\end{array}$ & High risk & Not randomized, single-group assignment \\
\hline $\begin{array}{l}\text { Incomplete outcome data } \\
\text { (attrition bias) } \\
\text { All outcomes }\end{array}$ & Low risk & 19 enrolled; 10 participants followed up at 12 weeks \\
\hline
\end{tabular}


NCT02648178 (Continued)

Selective reporting (reporting bias)

Unclear risk
The following measures were not reported: exhaled carbon dioxide; urine propylene glycol; urine nicotine, cotinine, NNAL and 1- hydroxy naphthalene (1-HOP), and Timeline Follow-Back Questionnaire (TLFB). Data at 6, 12 months also not reported

NCT02918630

\section{Study characteristics}

Methods

Design: RCT

Recruitment: Clinics

Setting: SMI clinics, USA

Study start date: October 2016; Study end date: August 2017

Participants

Total N: 7

N per arm: NRT: 4; EC+NRT 3

Inclusion criteria:

- Be diagnosed with schizophrenia (or other SMI, not clear)

- Be in stable medical condition (DSM-V)

- Report smoking $\geq 10$ tobacco cigarettes/day

- Present a breath $\mathrm{CO} \geq 10$ ppm

- Report wanting to reduce their cigarette smoking

- Be fluent in English

- Have a stable living situation

Exclusion criteria:

- Be currently pregnant or breastfeeding

- Report wanting to quit smoking in the immediate future

- Test positive for illicit drugs except THC

- Have any illness, medical condition, or use of medications, which in the opinion of the study physicians would preclude safe or successful completion of the study, or both

Inclusion based on specific population characteristic: Yes - SMI (schizophrenia and schizoaffective disorder, bipolar disorder, or PTSD)

43\% women; mean age 48.3; mean cpd: NR; mean FTND: NR

Motivated to quit: Wanted to quit or reduce their cigarette smoking but did not want to quit in the immediate future (this was an exclusion criterion) NB - trial registry states wanted to reduce and protocol states wanted to quit or reduce as inclusion criteria

E-cigarette use at baseline: Not specified

\section{EC: Refillable}

Both arms received a nicotine patch $21 \mathrm{mg}$ for 4 weeks

EC + NRT: 4 weeks: 1) a 3.3 V, 1000 mAh battery; and 2) a $1.5 \mathrm{Ohm}$, dual-coil cartomizer (SmokTech; Shenzhen, China). Nicotine concentrations $36 \mathrm{mg} / \mathrm{ml}$. Verbal and written instructions on how to use and maintain the e-cigarettes at Week 1 visit 
NCT02918630 (Continued)

NRT arm: NRT only

\begin{tabular}{|c|c|}
\hline Outcomes & $\begin{array}{l}5 \text { weeks } \\
\text { Cessation: } n / a \text { but "change in smoking" } \\
\text { Adverse events and biomarkers: } \\
\text { Breath CO, COPD-related symptoms, EC side effects (e-cig side effects questionnaire), AEs, SAEs } \\
\text { Other outcomes measured: } \\
\text { Urinary cotinine, cpd, tobacco dependence, craving, withdrawal symptoms, desire to quit, confidence } \\
\text { to quit, EC dependence, EC use, satisfaction with EC, nicotine dependence, schizophrenia symptoms } \\
\text { (brief psychiatric rating scale), cognitive domains associated with schizophrenia (MATRICS consensus } \\
\text { cognitive battery), changes in positive symptoms of schizophrenia (scale for the assessment of positive } \\
\text { symptoms), changes in negative schizophrenia symptoms (scale for the assessment of negative symp- } \\
\text { toms), suicide ideation (Columbia Suicide Severity Rating Scale) }\end{array}$ \\
\hline Study funding & Not reported \\
\hline Author declarations & Not reported \\
\hline Notes & $\begin{array}{l}\text { New for } 2020 \text { update. Information from http://clinical trials gov registry and unpublished protocol; dis- } \\
\text { crepancies between the two in terms of trial methods. Feasibility for future NIH grant application. In- } \\
\text { tended to recruit } 20 \text { participants but only } 7 \text { started and completed }\end{array}$ \\
\hline
\end{tabular}

\section{Risk of bias}

\begin{tabular}{|c|c|c|}
\hline Bias & Authors' judgement & Support for judgement \\
\hline $\begin{array}{l}\text { Random sequence genera- } \\
\text { tion (selection bias) }\end{array}$ & Unclear risk & Not reported \\
\hline $\begin{array}{l}\text { Allocation concealment } \\
\text { (selection bias) }\end{array}$ & Unclear risk & Not reported \\
\hline $\begin{array}{l}\text { Blinding of participants } \\
\text { and personnel (perfor- } \\
\text { mance bias) } \\
\text { All outcomes }\end{array}$ & Unclear risk & "double-blind" but "open-label" elsewhere, no further info given \\
\hline $\begin{array}{l}\text { Blinding of outcome as- } \\
\text { sessment (detection bias) } \\
\text { All outcomes }\end{array}$ & Unclear risk & Not reported \\
\hline $\begin{array}{l}\text { Incomplete outcome data } \\
\text { (attrition bias) } \\
\text { All outcomes }\end{array}$ & Unclear risk & Not reported \\
\hline $\begin{array}{l}\text { Selective reporting (re- } \\
\text { porting bias) }\end{array}$ & High risk & Schizophrenia and COPD outcomes not reported. \\
\hline Other bias & Unclear risk & $\begin{array}{l}\text { Some discrepancies between clinicaltrials record and protocol linked to from } \\
\text { record, including when NRT started and inclusion criteria (just schizophrenia } \\
\text { or all SMI). Target sample size was } 20 \text { but only } 7 \text { people recruited }\end{array}$ \\
\hline
\end{tabular}


Nides 2014

\section{Study characteristics}

\begin{tabular}{|c|c|}
\hline Methods & $\begin{array}{l}\text { Design: Open-label non-comparative study } \\
\text { Recruitment: Study site database and community advertisements } \\
\text { Setting: Clinical Trials Unit, USA } \\
\text { Study start date: April 2013; Study end date: } 10 \text { July } 2013\end{array}$ \\
\hline Participants & $\begin{array}{l}\text { Total N: } 29 \\
\text { Inclusion criteria: } \\
\text { - Age } 18 \text { - } 65 \text { years } \\
\text { - Good health } \\
\text { - BMI } 18 \text { - } 35 \\
\text { - Smoking } 10+\mathrm{cpd} \\
\text { - CO }>10 \text { ppm } \\
\text { Exclusion criteria: } \\
\text { - Pregnancy or breastfeeding } \\
\text { - Other drug dependency } \\
\text { - Use of any psychiatric or opioid medications } \\
\text { - EC within the previous } 14 \text { days } \\
\text { - Use of NRT in last } 30 \text { days } \\
\text { - Want to reduce or quit smoking within the next } 30 \text { days } \\
\text { Exclusion criterion: EC within the previous } 14 \text { days; use of NRT in last } 30 \text { days } \\
44 \% \text { women; mean age } 43 \text {; mean cpd } 20.1 \text {; mean FTND } 4.5 \\
\text { Motivated to quit: no } \\
\text { E-cigarette use at baseline }\end{array}$ \\
\hline
\end{tabular}

\section{EC: Cig-a-like}

Participants attended 3 clinic visits at 1-week intervals

Visit 1: Baseline

Visit 2: Provided with 1st generation type - 'NJOY ${ }^{\circledR}$ King Bold' (NJOY, Inc. Scottsdale, AZ), with 26 mg nicotine. Used ad libitum for 20 minutes in the clinic, then ad libitum use over the next week. Recorded use of regular cigarettes and puffs on EC

Visit 3: Participants abstained from all sources of nicotine for 12 hours prior to visit

\begin{tabular}{ll}
\hline Outcomes & Adverse events \\
\hline Study funding & Funding for this study was provided by NJOY, Inc., Scottsdale, AZ \\
\hline Author declarations & $\begin{array}{l}\text { Dr Nides has received compensation from NJOY, Inc. and GlaxoSmithKline. Dr Leischow has received } \\
\text { compensation from GlaxoSmithKline, Pfizer, and Cypress Bioscience. Mr Simmons and Ms Bhatter have } \\
\text { no conflict of interest to report }\end{array}$ \\
\hline
\end{tabular}

Notes

\section{Risk of bias}


Nides 2014 (Continued)

Bias Authors'judgement Support for judgement

Random sequence genera- High risk Prospective cohort

tion (selection bias)

Allocation concealment $\quad$ High risk $\quad$ Not randomized
(selection bias)

Incomplete outcome data Low risk $\quad 2$ participants dropped out between visits 1 and 2
(attrition bias)
All outcomes

Selective reporting (re- Low risk
porting bias)

\section{Oncken 2015}

\section{Study characteristics}

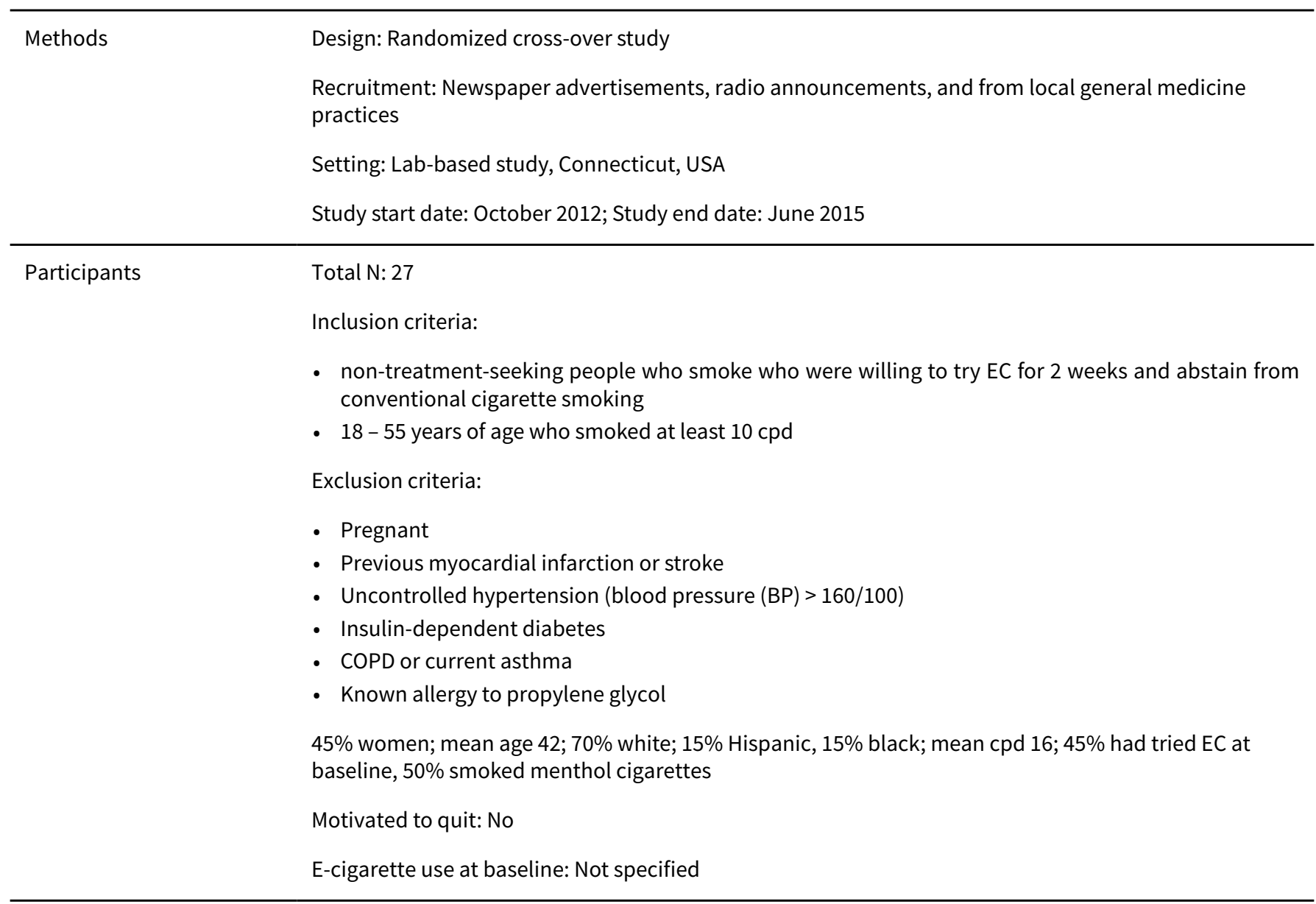

Interventions

\section{EC: Cig-a-like}

Prescribed Joye eGo-C (www.joyetech.com) and e-Juice ( $18 \mathrm{mg} / \mathrm{mL}$ nicotine) procured from American eLiquid (www.americanliquid.com) Cross-over study between menthol-flavored and non-menthol 
tobacco-flavored EC. Requested not to smoke their regular cigarettes during study period, but most

$(60 \%)$ reported intermittently smoking cigarettes during study

\begin{tabular}{|c|c|c|}
\hline \multirow[t]{4}{*}{ Outcomes } & \multicolumn{2}{|c|}{ Follow-up at 1 wk and 2 weeks } \\
\hline & \multicolumn{2}{|c|}{$\begin{array}{l}\mathrm{BP} \text {, heart rate, body plethysmography, static lung volumes and airways resistance (Raw) and specific } \\
\text { conductance ( } \mathrm{SGaw} \text { - taken at lab visits after abstaining from EC for at least } 2 \text { hrs, then taken again af- } \\
\text { ter inhaling EC and repeated } 5 \text { mins later }\end{array}$} \\
\hline & \multicolumn{2}{|c|}{ Adverse events also reported but method for measuring not stated } \\
\hline & \multicolumn{2}{|c|}{ Also measured nicotine concentrations, rates of cigarette and EC use } \\
\hline Study funding & \multicolumn{2}{|c|}{$\begin{array}{l}\text { This project was supported by Academic Enhancement funds from the Department of Medicine at the } \\
\text { University of Connecticut Health Center (to CO) and the Clinical Research Center at the University of } \\
\text { Connecticut Health Center }\end{array}$} \\
\hline Author declarations & \multicolumn{2}{|c|}{$\begin{array}{l}\text { CO is currently receiving study medication (nicotine inhaler and placebo) from Pfizer pharmaceuticals } \\
\text { for an NIH funded of nicotine inhaler for smoking cessation during pregnancy }\end{array}$} \\
\hline \multicolumn{3}{|l|}{ Notes } \\
\hline \multicolumn{3}{|l|}{ Risk of bias } \\
\hline Bias & Authors' judgement & Support for judgement \\
\hline \multirow{2}{*}{$\begin{array}{l}\text { Random sequence genera- } \\
\text { tion (selection bias) }\end{array}$} & \multirow[t]{2}{*}{ Unclear risk } & Method not stated; \\
\hline & & $\begin{array}{l}\text { Quote: "Subjects were then randomly assigned to use the menthol or plain e- } \\
\text { cigarette cartridge for one week, switching to the other cartridge for the sec- } \\
\text { ond week" }\end{array}$ \\
\hline $\begin{array}{l}\text { Allocation concealment } \\
\text { (selection bias) }\end{array}$ & Unclear risk & No detail given \\
\hline $\begin{array}{l}\text { Blinding of participants } \\
\text { and personnel (perfor- } \\
\text { mance bias) } \\
\text { All outcomes }\end{array}$ & Low risk & $\begin{array}{l}\text { No detail given on blinding but equal levels of support between arms, so per- } \\
\text { formance bias judged unlikely }\end{array}$ \\
\hline $\begin{array}{l}\text { Blinding of outcome as- } \\
\text { sessment (detection bias) } \\
\text { All outcomes }\end{array}$ & Low risk & $\begin{array}{l}\text { Some subjective outcomes but equal levels of support between arms so differ- } \\
\text { ential misreport judged unlikely }\end{array}$ \\
\hline $\begin{array}{l}\text { Incomplete outcome data } \\
\text { (attrition bias) } \\
\text { All outcomes }\end{array}$ & Low risk & $20 / 27$ followed up \\
\hline $\begin{array}{l}\text { Selective reporting (re- } \\
\text { porting bias) }\end{array}$ & Unclear risk & Unable to determine prespecified outcomes \\
\hline
\end{tabular}

\section{Ozga-Hess 2019}

\section{Study characteristics}

Methods Design: RCT


Ozga-Hess 2019 (Continued)

Recruitment: Cigarette smokers were recruited from the community via fliers, online postings, and word of mouth

Setting: Morgantown, West Virginia, USA

Study start date: Not reported. Study end date: Not reported

\section{Participants}

Total N: 60; E-cigarette plus own brand $=30$. Own brand cigarette $($ control $)=30$

38.3\% female; mean age completers 35.1 (SD 11) ( $N=34$ ) non-completers 36.8 (SD 12.9) ( $N=26)$; mean cpd completers 16.7 (SD 4.9), non-completers 19.6 (SD 6.1); mean FTND completers 5.3 (SD 1.8), noncompleters 5.9 (SD 1.9)

Inclusion criteria:

- 18 to 60 years of age; smoking $\geq 10$ cigarettes per day for $\geq 1$ year

- exhaled air carbon monoxide (CO) level of $\geq 10$ ppm (Micro+ ${ }^{\mathrm{TM}}$ basic monitor; CoVita; Haddonfield, $\mathrm{NJ}$ )

- Contemplation or Preparation Stage of Change (indicating interest in a quit attempt within the next 1 - 6 months)

Exclusion criteria:

- reported chronic health or psychiatric conditions

- past month use of marijuana $\geq 5$ days

- past month use of any other illicit drugs, or regular use of ECIGs or other tobacco products (i.e. $\geq 1$ day per week)

- individuals in the Precontemplation (no interest in quitting) or Action (actively trying to quit) Stage of Change

- currently breast- feeding or tested positive for pregnancy via urinalysis

\section{EC: Refillable}

E-cigarette $(18 \mathrm{mg} / \mathrm{ml})$ plus own brand cigarette. Kanger mini Protank-II, which is a $1.5 \mathrm{ml}$ Pyrex glass tank with a drip tip and atomizer head coils (KangerTech; China), and a $3.3 \mathrm{~V}$ constant output, $900 \mathrm{mAh}$, eGo-T battery (Joyetech; Irvine, CA). The liquid (The Vapor Room, Sky Vapors LLC, Frostburg, MD) was labeled as $70 \%$ propylene glycol and $30 \%$ vegetable glycerin, with a nicotine concentration requested of $18 \mathrm{mg} / \mathrm{ml}$. Participants could choose tobacco, menthol or wild berry flavor and could switch between sessions. Ad libitum use for 4 weeks

Own brand cigarette ad libitum use for 4 weeks

Outcomes Daily for salivary cotinine samples. Daily self-monitoring device to log e-cigarette and cigarette use. Collected used cigarette filters

Weekly CO breath test

Attended the laboratory weekly for assessments (Days 8, 15, 22, and 29). Then completed a follow-up visit 1-month post-intervention

self-reported withdrawal symptoms

Reported experience of specific symptoms rated using a visual analog scale with a range from 0 (not at all) to 100 (extremely). e.g. craving, irritability, dry mouth, throat irritation, and cough

Study funding

Financial support provided to MDB and GAD by WVU Senate Grant for Research, and to GAD, MDB, and NAT by Cooperative Agreement Number 1-U48-DP-005004 from the Centers for Disease Control and Prevention (CDC) to the West Virginia Prevention Research Center. Support provided to NJF and JEOH by the National Institute of General Medical Sciences (NIGMS T32 GM081741). Additional support provided by WV Tobacco Cessation QuitLine 
Ozga-Hess 2019 (Continued)

Author declarations

Author SGF has consulted for various pharmaceutical companies on matters relating to smoking cessation. All other authors declare that they have no conflicts of interest

Notes New for 2021 update

\section{Risk of bias}

\begin{tabular}{|c|c|c|}
\hline Bias & Authors' judgement & Support for judgement \\
\hline $\begin{array}{l}\text { Random sequence genera- } \\
\text { tion (selection bias) }\end{array}$ & Unclear risk & $\begin{array}{l}\text { Quote: "Using a simple randomized design" } \\
\text { Comment: not adequately explained }\end{array}$ \\
\hline $\begin{array}{l}\text { Allocation concealment } \\
\text { (selection bias) }\end{array}$ & Unclear risk & Not adequately described in the paper \\
\hline $\begin{array}{l}\text { Blinding of participants } \\
\text { and personnel (perfor- } \\
\text { mance bias) } \\
\text { All outcomes }\end{array}$ & High risk & Participants and investigators were not blind \\
\hline $\begin{array}{l}\text { Blinding of outcome as- } \\
\text { sessment (detection bias) } \\
\text { All outcomes }\end{array}$ & Low risk & Biochemical validation \\
\hline $\begin{array}{l}\text { Incomplete outcome data } \\
\text { (attrition bias) } \\
\text { All outcomes }\end{array}$ & High risk & $40 \%$ retention, but no difference between groups \\
\hline $\begin{array}{l}\text { Selective reporting (re- } \\
\text { porting bias) }\end{array}$ & Low risk & All outcomes reported \\
\hline
\end{tabular}

\section{Study characteristics}

\begin{tabular}{|c|c|}
\hline \multirow[t]{4}{*}{ Methods } & Design: Uncontrolled pre-post pilot study \\
\hline & Recruitment: Word of mouth \\
\hline & Setting: Hospital-based smoking cessation clinic, Italy \\
\hline & Study start date/end date: Not specified \\
\hline \multirow[t]{7}{*}{ Participants } & Total N: 34 \\
\hline & Inclusion criteria: \\
\hline & $\begin{array}{l}\text { - Adults who smoke, unwilling to quit smoking tobacco cigarettes and who have never tried a quit- } \\
\text { smoking protocol or have refused any smoking cessation treatment, or both }\end{array}$ \\
\hline & Exclusion criteria: \\
\hline & - None stated \\
\hline & Inclusion based on specific population characteristic: No \\
\hline & $47.1 \%$ women, mean age 40.6 , mean cpd 21.5 \\
\hline
\end{tabular}


Pacifici 2015 (Continued)

no EC use at baseline, not motivated to quit

Interventions

\section{EC: Refillable}

Participants were given commercially-available EC (AVATAR device, Battery $550 \mathrm{mAh} / 3.9 \mathrm{~V}, \mathrm{~W}: 7.8$, cartomizer with 2, 2 ohm resistance, tank capacity $1.5 \mathrm{~mL}$, temperature of the aerosol: 55/65 degrees), 2 different chargers for each EC and PUFFIT e-liquids with nicotine content matching the individual nicotine daily intake and tobacco and/or other flavors freely chosen by each participant

W1: nicotine-free e-liquid

W2\&3: Own EC with personal nicotine dosage, encouraged to use as substitute for traditional cigarettes

W4: Encouraged to forego all traditional cigarettes

Throughout: assistance at any time of day from centre staff with any EC-related problem, plus follow-up group sessions and smartphone messaging application

Behavioural support:

Multi-component medically-assisted training program with monitoring of nicotine intake as a biomarker of correct EC use, including Information about general working principles, safety and risks of EC, together with medically-assisted face-to-face training on how to correctly use the device to absorb nicotine vapor

\begin{tabular}{ll}
\hline Outcomes & Follow-up at 1,4 and $8 \mathrm{~m}$ \\
& Cessation (measure not defined) \\
& Adverse events \\
& Exhaled CO, COT, 3-HCOT concentration \\
& cpd \\
\hline Study funding & $\begin{array}{l}\text { The authors thank Renata Solimini, Adele Minutillo, Emilia Marchei and Maria Concetta Rotolo for their } \\
\text { technical assistance. This work was supported by the Department of Therapeutic Research and Medi- } \\
\text { cines Evaluation Istituto Superiore di Sanità, Roma, Italy }\end{array}$ \\
\hline Author declarations & The authors declare no conflict of interest \\
\hline Notes & \\
\hline
\end{tabular}

\section{Risk of bias}

\begin{tabular}{lll}
\hline Bias & Authors' judgement & Support for judgement \\
\hline $\begin{array}{l}\text { Random sequence genera- } \\
\text { tion (selection bias) }\end{array}$ & High risk & Not controlled \\
\hline $\begin{array}{l}\text { Allocation concealment } \\
\text { (selection bias) }\end{array}$ & High risk & Not controlled \\
\hline $\begin{array}{l}\text { Incomplete outcome data } \\
\text { (attrition bias) } \\
\text { All outcomes }\end{array}$ & Low risk & All participants followed up \\
\hline
\end{tabular}

Selective reporting (re- High risk
porting bias)


Polosa 2011

\section{Study characteristics}

\begin{tabular}{|c|c|}
\hline Methods & $\begin{array}{l}\text { Design: Prospective cohort } \\
\text { Recruitment: Advertisments in local hospital in Catania, Italy } \\
\text { Setting: not specified } \\
\text { Study start date: February 2010; Study end date: June } 2010\end{array}$ \\
\hline Participants & $\begin{array}{l}\text { Total } \mathrm{N} \text { : } 40 \text {, hospital staff } \\
\text { Inclusion criteria: } \\
\text { - Healthy people who smoke } \\
\text { - } 18 \text { - } 60 \text { years old } \\
\text { - } \text { smoking } \geq 15 \mathrm{cpd} \text { for at least the past } 10 \text { years, and not wanting to quit smoking at any time in the } \\
\text { next } 30 \text { days } \\
\text { Exclusion criteria: } \\
\text { - History of alcohol and illicit drug use } \\
\text { - Psychiatric illness } \\
\text { - Recent myocardial infarction } \\
\text { - Angina pectoris } \\
\text { - High blood pressure (BP > } 140 \text { mmHg systolic or } 90 \text { mmHg diastolic, or both) } \\
\text { - Diabetes mellitus } \\
\text { - Severe allergies } \\
\text { - Poorly-controlled asthma or other airways diseases } \\
\text { 35\% women, mean age } 42.9 \text { (SD } 8.8 \text { ), median cpd } 25 \text { (IQR } 20 \text { - 30), median FTND } 6.0 \text { (IQR } 6 \text { - 8) } \\
\text { Motivated to quit: No } \\
\text { E-cigarette use at baseline: Not specified }\end{array}$ \\
\hline
\end{tabular}

Interventions

\section{EC: Cig-a-like}

Seen at baseline, given EC ('Categoria' brand) with an initial 4-week supply of $7.4 \mathrm{mg}$ nicotine cartridges. Instructed to use ad libitum up to 4 cartridges per day. EC cartridges supplied at months 1, 2 , and 3

No instruction on cessation or reduction was provided

Outcomes

Follow-up at 1, 2, 3, 6, 18 and 24 months where cigarette consumption, $\mathrm{CO}$, and AEs were measured, incl. 30-day PP CO-validated abstinence at 6 months and CO-validated abstinence at 18 and 24 months (not otherwise defined)

Adverse events

Study funding

"We wish to thank Arbi Group Srl (Milano, Italy) for the free supplies of 'Categoria' e-Cigarette kits and nicotine cartridges as well as their support. We would also like to thank the study participants for all their time and effort and LIAF (Lega Italiana AntiFumo) for the collaboration"

Author declarations "None of the authors have any competing interests to declare, but RP has received lecture fees from
Pfizer and, from Feb 2011, he has been serving as a consultant for Arbi Group Srl.Arbi Group Srl (Milano,
Italy), the manufacturer of the e-Cigarette supplied the product, and unrestricted technical and cus-
tomer support. They were not involved in the study design, running of the study or analysis and presen-
tation of the data" 
Polosa 2011 (Continued)

Notes Smoking cessation services provided to those who spontaneously asked for assistance with quitting.

These participants were excluded from the study protocol

\section{Risk of bias}

\begin{tabular}{|c|c|c|}
\hline Bias & Authors' judgement & Support for judgement \\
\hline $\begin{array}{l}\text { Random sequence genera- } \\
\text { tion (selection bias) }\end{array}$ & High risk & Prospective cohort \\
\hline $\begin{array}{l}\text { Allocation concealment } \\
\text { (selection bias) }\end{array}$ & High risk & Not randomized \\
\hline $\begin{array}{l}\text { Incomplete outcome data } \\
\text { (attrition bias) } \\
\text { All outcomes }\end{array}$ & Low risk & $13 / 40$ were lost to follow-up, but used ITT analysis \\
\hline $\begin{array}{l}\text { Selective reporting (re- } \\
\text { porting bias) }\end{array}$ & Unclear risk & Unable to determine prespecified outcomes \\
\hline
\end{tabular}

Polosa 2014b

\section{Study characteristics}

\begin{tabular}{ll}
\hline Methods & Design: Prospective cohort study \\
Recruitment: Volunteers, leaflets, cessation service kiosk in hospital \\
Setting: Smoking cessation clinic, Italy \\
Study start date: January 2013; Study end date: November 2013 \\
\hline Total N: 50 \\
Inclusion criteria: \\
- Healthy people who smoke \\
- $18-60$ years old \\
- Smoking $\geq 15$ conventional cpd for at least 10 years \\
Exclusion criteria: \\
- none stated \\
$40 \%$ women, mean age 41 , mean cpd 25, mean FTND 6.0 \\
No EC use at baseline, not motivated to quit
\end{tabular}

\section{EC: Refillable}

2nd generation devices (personal vaporisers - PVs): EGO/CE4 model, filled with tobacco aroma e-Liquid containing $9 \mathrm{mg} / \mathrm{ml}$ nicotine; instructed to use the study products ad libitum (up to a maximum of $5 \mathrm{ml}$ ) day; i.e. half vial)

Behavioural support: 
Polosa 2014b (Continued)

Participants were instructed how to charge, fill, activate and use the EC. Key troubleshooting was addressed and phone numbers were supplied for assistance. "No emphasis on encouragement, motivation and reward for the smoking cessation-related efforts were provided during the study."

\begin{tabular}{|c|c|c|}
\hline \multirow[t]{4}{*}{ Outcomes } & \multicolumn{2}{|l|}{$4,8,12$ and 24 weeks } \\
\hline & \multicolumn{2}{|c|}{ 30-day PP verified by $\mathrm{CO} \leq 10 \mathrm{ppm}$} \\
\hline & \multicolumn{2}{|l|}{ Adverse events } \\
\hline & \multicolumn{2}{|c|}{$\mathrm{Cpd}$, exhaled CO, reduction rates, product usage, and opinions of the EC products } \\
\hline Study funding & \multicolumn{2}{|c|}{$\begin{array}{l}\text { "The authors wish to thank FlavourArt (Oleggio, NO, Italy; www.flavourart.it). Authors wish to thank } \\
\text { LIAF, Lega Italiana Anti Fumo (Italian acronym for Italian Anti Smoking League) for supporting this re- } \\
\text { search" }\end{array}$} \\
\hline Author declarations & \multirow{2}{*}{\multicolumn{2}{|c|}{$\begin{array}{l}\text { "RP has received lecture fees and research funding from Pfizer and GlaxoSmithKline, manufacturers } \\
\text { of stop smoking medications. He has also served as a consultant for Pfizer and Arbi Group Srl, an Ital- } \\
\text { ian distributor of e-Cigarettes. RP is currently scientific advisor for LIAF, Lega Italiana Anti Fumo (Italian } \\
\text { acronym for Italian Anti Smoking League). PC, MM, JBM, and CR have no relevant competing interest to } \\
\text { declare in relation to this }\end{array}$}} \\
\hline & & \\
\hline \multicolumn{3}{|l|}{ Notes } \\
\hline \multicolumn{3}{|l|}{ Risk of bias } \\
\hline Bias & Authors' judgement & Support for judgement \\
\hline $\begin{array}{l}\text { Random sequence genera- } \\
\text { tion (selection bias) }\end{array}$ & High risk & Not controlled \\
\hline $\begin{array}{l}\text { Allocation concealment } \\
\text { (selection bias) }\end{array}$ & High risk & Not controlled \\
\hline $\begin{array}{l}\text { Incomplete outcome data } \\
\text { (attrition bias) } \\
\text { All outcomes }\end{array}$ & Low risk & $\begin{array}{l}76 \% \text { followed up, ITT analysis used, no significant differences in baseline char- } \\
\text { acteristics between completers and those lost to follow-up }\end{array}$ \\
\hline $\begin{array}{l}\text { Selective reporting (re- } \\
\text { porting bias) }\end{array}$ & Unclear risk & Unable to determine prespecified outcomes \\
\hline
\end{tabular}

Polosa 2015

\section{Study characteristics}

\begin{tabular}{ll}
\hline Methods & Design: Prospective cohort \\
& Recruitment: Professional retail staff in participating vape shops \\
& Setting: 7 vape shops in Catania province, Italy \\
& Study start date/end date: Not specified \\
\hline Participants & Total $\mathrm{N}: 71$ \\
Inclusion criteria:
\end{tabular}


Polosa 2015 (Continued)

- Adults who smoke ( $\geq 18)$

- making first purchase at participating vape shop (definition of smoker not stated)

Exclusion criteria:

- none stated

$38 \%$ women, mean age 41.7 , mean cpd 24.9, mean FTND 5

No EC use at baseline

\begin{tabular}{ll}
\hline Interventions & EC: Refillable \\
& $\begin{array}{l}\text { Instructed how to charge, fill, activate and use EC; key troubleshooting advice provided; phone number } \\
\text { available for technical support "Encouraged to use these products in anticipation of reducing the num- } \\
\text { ber of cig/day smoked" }\end{array}$ \\
\hline Outcomes & and 12 m follow-up \\
& 30-day PPA via self-report \\
& Details of product purchase \\
& Sustained $50 \%$ and $80 \%$ reduction in cpd from baseline \\
\hline Study funding & $\begin{array}{l}\text { Authors wish to thank the local participating Vape Shops and LIAF, Lega Italiana Anti Fumo (Italian } \\
\text { acronym for the Italian Anti-Smoking League) for supporting this research }\end{array}$ \\
\hline Author declarations & $\begin{array}{l}\text { Riccardo Polosa has received lecture fees and research funding from Pfizer and GlaxoSmithKline, man- } \\
\text { ufacturers of stop smoking medications. He has also served as a consultant for Pfizer and Arbi Group } \\
\text { Srl, an Italian distributor of e-Cigarettes. Riccardo Polosa is currently scientific advisor for LIAF, Lega } \\
\text { Italiana Anti Fumo (Italian acronym for Italian Anti-Smoking League). Jacques Le-Houezec is a consul- } \\
\text { tant for Johnson \& Johnson France, a manufacturer of nicotine replacement therapy, and was reim- } \\
\text { bursed for travel and accommodation to present at a conference in Shenzhen (China) organized by the } \\
\text { e-cig manufacturer association (CECMOL). Pasquale Caponnetto and Fabio Cibella have no relevant } \\
\text { conflict of interest to declare in relation to this work }\end{array}$ \\
\hline
\end{tabular}

Notes

Risk of bias

\begin{tabular}{lll}
\hline Bias & Authors' judgement & Support for judgement \\
\hline $\begin{array}{l}\text { Random sequence genera- } \\
\text { tion (selection bias) }\end{array}$ & High risk & Not controlled \\
\hline $\begin{array}{l}\text { Allocation concealment } \\
\text { (selection bias) }\end{array}$ & High risk & Not controlled \\
\hline $\begin{array}{l}\text { Incomplete outcome data } \\
\text { (attrition bias) }\end{array}$ & Low risk & $\begin{array}{l}\text { 69\% follow-up at 12 m. Participants lost to follow-up considered as continuing } \\
\text { All outcomes }\end{array}$ \\
\hline $\begin{array}{l}\text { Selective reporting (re- } \\
\text { porting bias) }\end{array}$ & Unclear risk & Unable to determine prespecified outcomes \\
\hline
\end{tabular}


Pratt 2016

\section{Study characteristics}

Design: Observational study - uncontrolled experimental study
Recruitment: community mental health centre through self-referral and clinician referrals
Setting: community mental health centre (USA)
Study start date: October 2013; Study end date: June 2014

Participants Total N: 19 (21 originally recruited, however 2 participants did not return for any weekly visits so 19 analyzed)

Inclusion criteria:

- Age $\geq 18$

- Primary DSM-IV axis I diagnosis, based on chart review and confirmation by the community mental health centre team psychiatrist, of schizophrenia, schizoaffective disorder, or bipolar disorder

- SMI defined by at least moderate impairment in multiple domains of life functioning due to mental illness

- Smoking at least 10 cigarettes per day

- History of failed treatment-facilitated quit attempts

- Voluntary informed consent for participation

Exclusion criteria:

- Current use of e-cigarettes

- Medical instability

- Primary diagnosis of dementia or significant cognitive impairment defined as a Mini Mental Status Examination (MMSE) score $<24$

Inclusion based on specific population characteristic: Psychiatrically stable, in-treatment, people who smoke with a schizophrenia spectrum disorder or bipolar disorder

$68 \%$ women; mean age 42; mean cpd: Only cigarettes per week reported: $192(S D=159.3)$. This would be an average of $27 \mathrm{cpd}$; mean FTND 5.5

Motivated to quit: "None of the participants was actively engaged in a quit attempt during the study"

E-cigarette use at baseline: E-cig use was an exclusion criterion

\section{EC: Cig-a-like}

E-cigarette details: (NJOY brand) based on each participant's level of use of combustible tobacco. Each e-cigarette cartridge was approximately equivalent to 2 packs of combustible cigarettes. Trained research interviewers instructed participants on the proper use of e-cigarettes

Outcomes

Week 1, 2, 3, 4

Adverse events and biomarkers:

- Breath CO level

- Possible side effects

Other outcomes measured:

- Use of tobacco products

- Fagerström nicotine dependence scores

- Appeal of EC

- Level of enjoyment of EC

- Satisfaction with EC compared with usual combustible tobacco 
Pratt 2016 (Continued)

- Willingness to purchase EC

Study funding

"Financial support to purchase the e-cigarettes and pay small stipends to the participants in this unfunded pilot study came from Dr. Mary Brunette's discretionary reserve account."

Author declarations "All authors declare that they have no conflicts of interest"

Notes

\section{Risk of bias}

\begin{tabular}{lll}
\hline Bias & Authors' judgement & Support for judgement \\
\hline $\begin{array}{l}\text { Random sequence genera- } \\
\text { tion (selection bias) }\end{array}$ & High risk & Not randomized \\
\hline $\begin{array}{l}\text { Allocation concealment } \\
\text { (selection bias) }\end{array}$ & High risk & Not randomized \\
\hline $\begin{array}{l}\text { Incomplete outcome data } \\
\text { (attrition bias) } \\
\text { All outcomes }\end{array}$ & Low risk & 2 dropouts (9.5\%) failed to return to clinic. Analysis based on 19 participants \\
\hline $\begin{array}{l}\text { Selective reporting (re- } \\
\text { porting bias) }\end{array}$ & Low risk & All expected outcomes reported \\
\hline
\end{tabular}

\section{Pulvers 2018}

\section{Study characteristics}

\begin{tabular}{l} 
Design: Observational uncontrolled experimental study \\
Recruitment: Community \\
Setting: Visits took place in University labs, USA \\
Study start date: January 2015; Study end date: April 2015 \\
\hline Total N: 40 \\
Inclusion criteria: \\
- Being 18 years of age or older \\
- Cigarette smoking on at least 4 days of the past 30 days for at least 1 year \\
- Never using EC regularly (less than 25 lifetime uses) \\
- Not having used EC on more than 3 of the past 30 days \\
- Being willing to switch from smoking regular cigarettes to ECs \\
- Fluency in English \\
- Having regular access to a telephone and transportation to attend appointments \\
- Being willing to abstain from using marijuana during the study \\
Exclusion criteria: \\
- Any use of other tobacco products (OTPs) including smokeless tobacco, cigarillos, pipes, cigars, hand- \\
rolled cigarettes, and hookah in the past 30 days
\end{tabular}


- Past 30 day use of nicotine replacement therapy or medication which aids smoking cessation including bupropion, clonidine, nortriptyline, or varenicline

- Having uncontrolled asthma, severe allergies, or diabetes mellitus

- Currently taking prescription medication for emotional distress, depression, or other psychological problems

- Current dependence on a substance other than nicotine

- Presence of any cardiovascular or pulmonary illnesses in the past 6 months

- For women, pregnancy or plans to become pregnant in the next 6 months

Inclusion based on specific population characteristic: No

27\% women; mean age 30.08; mean cpd 8.76; FTND not reported

Motivated to quit: over half either did not intend to quit at all or did not intend to quit in the next 6 months $22 / 40(55 \%)$

E-cigarette use at baseline: Inclusion criteria included the following:

- Never using EC regularly (less than 25 lifetime uses)

- Not having used EC on more than 3 of the past 30 days

Interventions

\section{EC: Refillable}

2nd generation EC starter kit with $2 \mathrm{e}-\mathrm{Go} \mathrm{C}$ batteries ( 3.7 volts/650 MaH), a USB connection cord, an $\mathrm{AC}$ adapter, and a carrying case, and a supply of Saturn V4i atomizers ( $2.4 \mathrm{ohms}$ ) filled with liquid in their preferred flavor (28 atomizers total; $2 /$ day). Provided $24 \mathrm{mg} / \mathrm{mL}$ dosage vegetable glycerin liquid in a tester sample to all participants. Those who reported the $24 \mathrm{mg}$ was too strong were provided 12 $\mathrm{mg} / \mathrm{mL}$ dosage liquid. The first session included brief education, training, action planning for making a complete switch to EC. A referral to the California Smokers' Helpline was made at the final visit (week 4).

Outcomes

3 lab visits (baseline, week 2, and week 4) and 2 phone visits (week 1 and week 3). Biological samples were taken at all 3 in-person visits (baseline, week 2, and week 4). However, due to budgetary restrictions, only the baseline and week 4 biological data were analyzed

Adverse events and biomarkers:

- Biochemical measures only: Breath samples were taken with a Micro + (Bedfont, Haddonfield, NJ) to measure CO

- Urine samples taken to test for change in tobacco toxicant exposure by following measures:

* concentrations of NNAL measured by liquid chromatography-tandem mass spectrometry (LC-MS/ MS)

* metabolites of a panel of potentially toxic VOCs, including benzene (PMA), ethylene oxide (HEMA), $\mathrm{N}$-nitrosodimethylamine (MMA), acrylonitrile (CNEMA), acrolein(3-HPMA), propylene oxide (2-HPMA), acrylamide (AAMA), and crotonaldehyde (HPMMA) measured by LC-MS/MS,2

Other outcomes measured:

Cotinine, change in tobacco consumption (cpd using TLFB interview), change in frequency of EC use, change in nicotine dependence and attitudes/behavior, change in 30-day nicotine exposure

Study funding

Author declarations
"This study was funded by the University of Minnesota (JSA), P30 DA012393 (NLB), P50 CA180890 (NLB), and California State University San Marcos (KP)."

"Benowitz is a consultant to pharmaceutical companies that market smoking cessation medications and has been an expert witness in litigation against tobacco companies. The other authors have no conflicts of interest."

Notes New for 2020 update


Pulvers 2018 (Continued)

Risk of bias

\begin{tabular}{lll}
\hline Bias & Authors' judgement & Support for judgement \\
\hline $\begin{array}{l}\text { Random sequence genera- } \\
\text { tion (selection bias) }\end{array}$ & High risk & Not randomized \\
\hline $\begin{array}{l}\text { Allocation concealment } \\
\text { (selection bias) }\end{array}$ & High risk & Not randomized \\
\hline $\begin{array}{l}\text { Incomplete outcome data } \\
\text { (attrition bias) } \\
\text { All outcomes }\end{array}$ & Low risk & $37 / 40$ provided follow-up data \\
\hline $\begin{array}{l}\text { Selective reporting (re- } \\
\text { porting bias) }\end{array}$ & Low risk & All outcomes reported \\
\hline
\end{tabular}

Pulvers 2020

\section{Study characteristics}

Methods Design: RCT. Unblinded. 2:1 ratio

Recruitment: Participants were recruited from the San Diego, California, and Kansas City, Missouri and Kansas, metropolitan areas

Setting: USA

Study start date: May 2018. Study end date: May 2019

Participants Total $\mathrm{N}=186$; Electronic-cigarettes $=125$. Own brand cigarette $=61$

40.3\% female; mean age 43.3 (SD 12.5); mean cpd 12.1 (SD 7.2). E-cigarettes use at baseline: 0.05 (0.3\%)

Inclusion criteria:

- $>21$ years of age

- Smoked cigarettes on $>25$ of past 30 days

- Smoked $>5$ cigarettes per day on days smoked

- Smoked cigarettes $>6$ months

- Carbon monoxide > 5 PPM at baseline

- Systolic BP of $<160 \mathrm{mmHg}$ and diastolic BP of $<105 \mathrm{mmHg}$ at baseline

- Hispanic/Latino or African American/Black

- Fluent in English or Spanish

- Willing to switch from smoking cigarettes to ECs for 6 weeks

- Regular access to telephone

- Transportation to attend appointments (KC Only)

Exclusion criteria:

- Primary use of other tobacco products or equal use of cigarettes and other tobacco products

- Electronic cigarette use on $>4$ of the past 30 days

- Currently in a smoking cessation program or another clinical trial

- Use of nicotine replacement therapy or medication which aids smoking cessation in the past 30 days

- Hospitalization for a psychiatric issue in the past 30 days 
- Heart-related event in the past 30 days. Examples include heart attack, stroke, severe angina (i.e. chest pain), ischemic heart disease, and vascular disease

- Uncontrolled blood pressure at baseline

- Planning to move out of study centers (San Diego or Kansas City) in the next 6 weeks

- Another person in the household enrolled in the study

- Women: pregnant, breast-feeding, or planning to become pregnant in the next 6 months

- Unstable mental status or health status

Interventions

EC: pod

Electronic-cigarettes: JUUL ( $5 \%$ nicotine); Choice of flavors (Menthol, Mango, Cool Mint, Virginia Tobacco); Given 1 pod per pack of cigarettes; Given a 2-week supply at baseline and then a further 4-week supply at week-2 visit. At each follow-up appointment (week 1, telephone call; week 2, in-person visit; and week 4, telephone call), barriers and benefits of switching to e-cigarette were discussed and action planning for exclusive switching was revisited. Compensated on a schedule of USD 20 at baseline, USD 40 at week 2 and USD 60 at week 6

Own brand cigarettes: Compensated on a schedule of USD 20 at baseline, USD 40 at week 2 and USD 60 at week 6

Baseline, week 2 and week 6 . Telephone survey at 6 months
Change in past 7-day combustible cigarette use measured by 7-day timeline follow-back interview
30-day point prevalence at 6 months (EC group only)
- reduction in toxicant exposure, as measured by NNAL excretion.
- Cotinine
- CO

Lung function; Pulmonary function test of small airway disease that is most sensitive to effects of cigarette smoking; mean midexpiratory phase of forced expiratory (FEF25\%-75\%); respiratory symptoms as measured with the American Thoracic Society Questionnaire (scores range from 0 - 32, with higher scores indicating greater respiratory symptoms)

Blood pressure

Adverse events: respiratory symptoms

Study funding Drs Pulvers and Nollen and Ms Rice were supported by grant No. 5SC3GM122628 from the National Institutes of Health (NIH). Drs Schmid and Ahluwalia were supported in part by grant No. P20GM130414, from the NIH-funded Center of Biomedical Research Excellence (COBRE). Dr Schmid was partially supported by Institutional Development Award No. U54GM115677 from the National Institute of General Medical Sciences of the NIH, which funds Advance Clinical and Translational Research (Advance-CTR)

Author declarations

Dr Schmid reported serving as a consultant for legal firms representing Eli Lilly, Boehringer-Ingelheim, and Gilead outside the submitted work. Dr Benowitz reported receiving personal fees from Pfizer and Achieve Life Sciences and serving as a consultant to pharmaceutical companies that market smoking cessation medications and as an expert witness in litigation against tobacco companies outside the submitted work. Dr Ahluwalia reported receiving personal fees from Lucy Goods outside the submitted work. No other disclosures were reported.

Notes New for 2021 update. Additional data provided by authors

\section{Risk of bias}




\section{Pulvers 2020 (Continued)}

Random sequence genera- Low risk Randomization sequence was generated with an Excel (Microsoft) random tion (selection bias) number formula applied to each site (2:1 ratio)

\section{Allocation concealment Low risk} (selection bias)

Allocation was placed into sealed individual envelopes labeled with participant identification numbers for each site, retrieved from a locked cabinet monitored by the project manager, and opened individually following consent of each participant

Blinding of participants High risk
and personnel (perfor-

\begin{tabular}{|c|c|c|}
\hline $\begin{array}{l}\text { Blinding of outcome as- } \\
\text { sessment (detection bias) } \\
\text { All outcomes }\end{array}$ & Low risk & Carbon monoxide validation \\
\hline $\begin{array}{l}\text { Incomplete outcome data } \\
\text { (attrition bias) } \\
\text { All outcomes }\end{array}$ & Low risk & $\begin{array}{l}\text { E-cig: } 115 / 126 \\
\text { OB: } 54 / 61\end{array}$ \\
\hline $\begin{array}{l}\text { Selective reporting (re- } \\
\text { porting bias) }\end{array}$ & Low risk & Per protocol reporting \\
\hline
\end{tabular}

Scheibein 2020

\section{Study characteristics}

\begin{tabular}{|c|c|}
\hline \multirow[t]{4}{*}{ Methods } & Design: Non-randomized single-arm \\
\hline & $\begin{array}{l}\text { Recruitment: From supported temporary accommodation (STA) service STA project workers and sup- } \\
\text { port staff identified potential study participants who smoked and wished to quit }\end{array}$ \\
\hline & Setting: Dublin Simon Community, Ireland \\
\hline & $\begin{array}{l}\text { Study start date: Recruitment February } 2019 \text { (overall trial start date March 2018). Study end date: June } \\
2019\end{array}$ \\
\hline \multirow[t]{10}{*}{ Participants } & $\begin{array}{l}\text { Total N: } 23 \text { but only report baseline for the } 9 \text { that completed the study. \% female } 8.7 \%(2 / 23) \text { at base- } \\
\text { line, }(22.2 \% \text { 2/9) completed and reported; mean age } 43.89 \text { (SD 7.36); mean cpd } 25.22 \text { (SD 7.77); mean } \\
\text { FTND } 7.89 \text { (SD 1.2); mean CO } 21.89 \text { (SD } 14.4 \text { corresp) }\end{array}$ \\
\hline & E-cigarettes use at baseline: no \\
\hline & Motivated to quit: yes \\
\hline & Inclusion criteria: \\
\hline & - > 5 CO ppm (carbon monoxide) \\
\hline & - Active smoking status \\
\hline & - Expressed intention to quit using ENDS-device \\
\hline & Exclusion criteria: \\
\hline & - Self-reported pregnancy \\
\hline & $\begin{array}{l}\text { - Exhibition of florid psychotic or substance use-related symptoms which could have affected ability } \\
\text { to consent }\end{array}$ \\
\hline
\end{tabular}


Scheibein 2020 (Continued)

Interventions

\section{EC: Refillable}

Electronic-cigarette: Endura T22e Electronic Nicotine Delivery System and $210 \mathrm{ml}$ bottles of fluid strengths $(0,6,11,18$ and $20 \mathrm{mg} / \mathrm{ml}$ ) and flavors ('Purple Berry', 'Ice Menthol', 'Regular Blend' and 'American Tobacco')

Outcomes Baseline ('week 1'), week 4, week 8, week 12: CO, adverse events

Also number of cigarettes smoked; Fagerström Test Scores

Study funding

This study was completed as part of a Tobacco Harm Reduction Scholarship funded by Knowledge Action Change

Author declarations

FS was a recipient of a Tobacco Harm Reduction Scholarship provided by Knowledge Action Change. $\mathrm{He}$ is currently the recipient of an Enhanced Scholarship from the same organization. AM and KM acted as mentors for both the Tobacco Harm Reduction Scholarship and Enhanced Scholarship.

AM is an associate of New Nicotine Alliance.

KM is a recipient of a grant from the Foundation for a Smoke Free World.

JW declares no interests.

WR declares no interests

Notes New for 2021 update

\section{Risk of bias}

\begin{tabular}{|c|c|c|}
\hline Bias & Authors' judgement & Support for judgement \\
\hline $\begin{array}{l}\text { Random sequence genera- } \\
\text { tion (selection bias) }\end{array}$ & High risk & Only 1 arm \\
\hline $\begin{array}{l}\text { Allocation concealment } \\
\text { (selection bias) }\end{array}$ & High risk & Not randomized \\
\hline $\begin{array}{l}\text { Blinding of participants } \\
\text { and personnel (perfor- } \\
\text { mance bias) } \\
\text { All outcomes }\end{array}$ & High risk & Not randomized \\
\hline $\begin{array}{l}\text { Blinding of outcome as- } \\
\text { sessment (detection bias) } \\
\text { All outcomes }\end{array}$ & High risk & Self-reported adverse events \\
\hline $\begin{array}{l}\text { Incomplete outcome data } \\
\text { (attrition bias) } \\
\text { All outcomes }\end{array}$ & High risk & $\begin{array}{l}\text { 9/23 completed. Reason was many people moved away so not linked to unac- } \\
\text { ceptability of the study. Incomplete paperwork to enable to be followed }\end{array}$ \\
\hline $\begin{array}{l}\text { Selective reporting (re- } \\
\text { porting bias) }\end{array}$ & Unclear risk & Protocol published afterwards \\
\hline
\end{tabular}

Smith 2020

\section{Study characteristics}

Electronic cigarettes for smoking cessation (Review) 
Smith 2020 (Continued)

Methods
Design: Double-blind randomized controlled trial

Recruitment: Recruited from the local area via advertising on craigslist social media

Setting: Laboratory and electronic diaries, USA

Study start date/Study end date: Not specified.

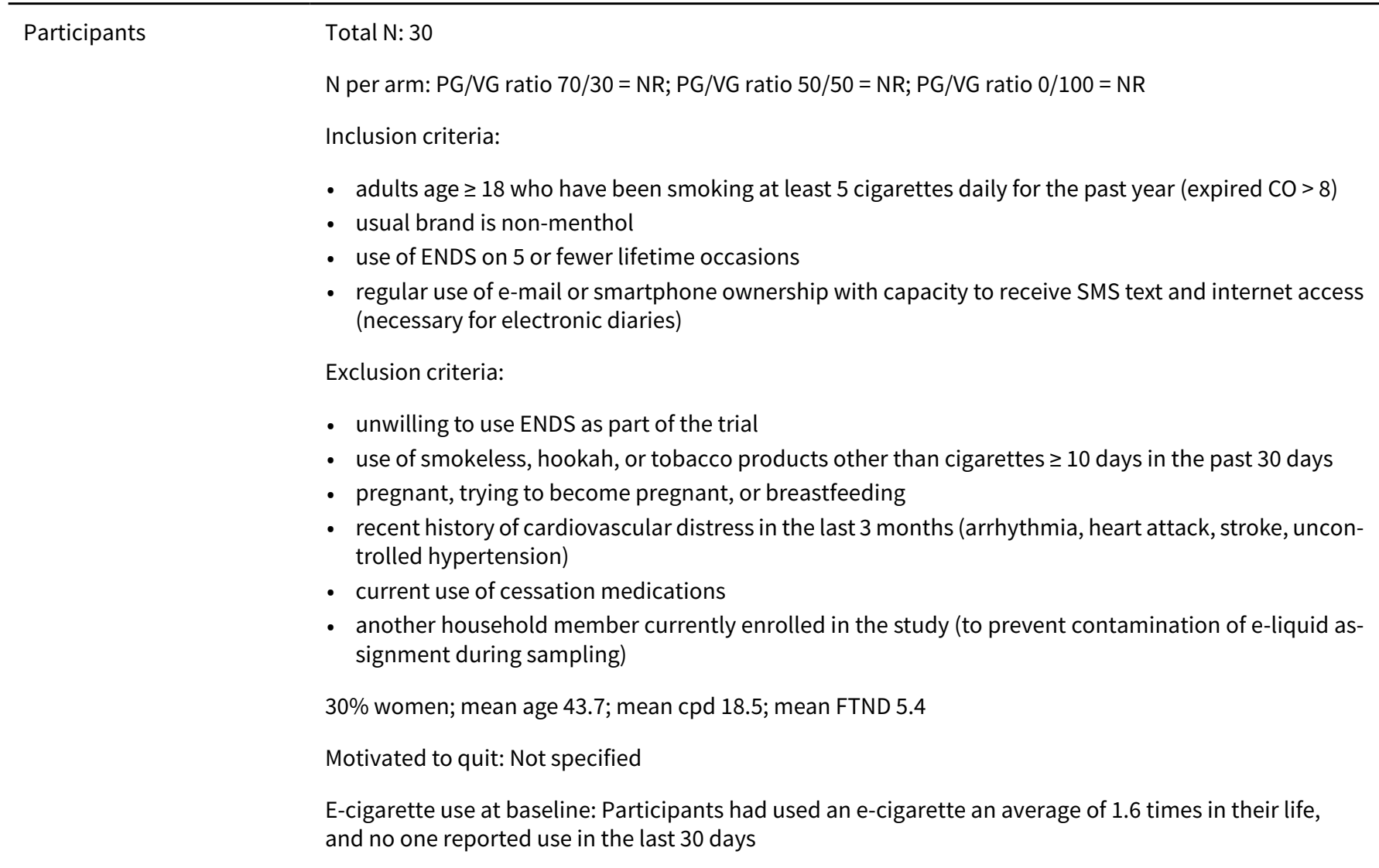

Interventions

\section{EC: Cig-a-like}

EC provided for 1 week. All aspects of the ENDS device and e-liquid were held constant between groups with the exception of $P G / V G$ ratio:

PG/VG ratio 70/30; PG/VG ratio 50/50; PG/VG ratio 0/100. Ego-T $1100 \mathrm{mAh}$ battery and disposable cartomizers (510 Smoketech, 1.5- $\Omega$ dual coil). E-liquid was tobacco-flavored (Classic Tobacco, American E-liquid) and contained $18 \mathrm{mg}$ nicotine $/ \mathrm{ml}$

\begin{tabular}{ll} 
Outcomes & 1 week; 2 lab visits pre and post and participant diaries \\
& Adverse events and biomarkers: Participants provided a CO sample at each visit \\
& Other outcomes measured: cpd, ENDS puffs \\
\hline Study funding & $\begin{array}{l}\text { Funding for this project was provided by pilot funding from the National Cancer Institute (P01CA200512 } \\
\text { to K.M.C.). Salary support provided by the National Institute on Drug Abuse (K12DA031794 to T.T.S., } \\
\text { K23DA041616 to B.W.H.) }\end{array}$ \\
\hline Author declarations & $\begin{array}{l}\text { M.J.C. has received consulting honoraria from Pfizer. K.M.C. has received payment as a consultant to } \\
\text { Pfizer, Inc., for service on an external advisory panel to assess ways to improve smoking cessation de- } \\
\text { livery in health care settings. He also has served as paid expert witness in litigation filed against the to- } \\
\text { bacco industry }\end{array}$
\end{tabular}


Smith 2020 (Continued)

Notes Additional data provided from authors. New for 2020 update.

\section{Risk of bias}

Bias Authors' judgement Support for judgement

Random sequence genera- Unclear risk tion (selection bias)

Quote: "At the conclusion of the lab visit, participants were randomized and assigned to take home one of the three e-liquids to use at home for a 1-week sampling period (10 participants/ratio)."

Quote: "Participants were randomly assigned to receive one e-liquid to take home for 1 week." (no further detail given)

\begin{tabular}{lll}
\hline $\begin{array}{l}\text { Allocation concealment } \\
\text { (selection bias) }\end{array}$ & Unclear risk & Not specified \\
\hline $\begin{array}{l}\text { Blinding of participants } \\
\text { and personnel (perfor- } \\
\text { mance bias) }\end{array}$ & Low risk & $\begin{array}{l}\text { Quote: "PG/VG ratio was blinded from participant and staff members who con- } \\
\text { ducted experimental sessions." }\end{array}$ \\
All outcomes & \\
\hline
\end{tabular}

\begin{tabular}{lll}
\hline Blinding of outcome as- $\quad$ Low risk $\quad$ Biochemical validation
\end{tabular}
sessment (detection bias)

All outcomes

Incomplete outcome data Low risk (attrition bias)

All outcomes
Selective reporting (re- Unclear risk porting bias)
Number of participants at follow-up not reported, but this may be due to the 1 -week follow-up and it seems that all participants (excluding 1 participant who was not randomized) were followed up

\section{Stein 2016}

\section{Study characteristics}

\begin{tabular}{ll}
\hline Methods & Design: Non-controlled open-label experimental study \\
Recruitment: A flyer posted at a large methadone maintenance treatment program \\
Setting: Methadone maintenance treatment program, USA \\
Study start date: April 2015; Study end date: Not specified \\
\hline Total N: 12 \\
Inclusion criteria: \\
- current moderate or heavy cigarette use (10+ cpd for at least 12 months prior to enrolment) \\
- current MMT for at least 3 months \\
- ready to make a smoking quit attempt in the next 14 days \\
- plan to remain on MMT for at least 12 weeks \\
Exclusion criteria: \\
- used e-cigarettes on more than 2 of the past 30 days \\
- currently used medications that may reduce smoking (bupropion, varenicline, NRT)
\end{tabular}
each group, but mean $\mathrm{CO}$ data provided by author on request 
Stein 2016 (Continued)

- had unstable medical or psychiatric conditions (past-month suicidal ideation or past-year suicide attempt, hospitalization for myocardial infarction or stroke in the prior 3 months)

- had regular use of marijuana (self-report or positive urine drug test)

Inclusion based on specific population characteristic: People receiving MMT for opoid use disorder

50\% women; mean age 45.9; mean cpd 17.8; mean FTND: Not reported

Motivated to quit: yes

E-cigarette use at baseline: Had not used e-cigarettes for more than 2 of the past 30 days

EC: Cig-a-like
$\begin{aligned} & 2 \text { week supply of NJOY e-cigarettes at week } 1 \text { (quit day), consisting of } 5 \text { packs of NJOY e-cigarettes (15 } \\ & \text { in total). Participants could request an additional } 5 \text { pack ( } 20 \text { in total) for the following } 2 \text {-week study } \\ & \text { period, if they ran out before a study visit. Participants instructed to use EC exclusively for a total of } 6 \\ & \text { weeks (end of treatment). They were referred to the state telephone QuitLine for supportive counseling } \\ & \text { at the quit-day visit (week } 1 \text { ) }\end{aligned}$

Participants quit and received e-cigs at week 1. Assessments were carried out at week 3, 5, 7 and 9
Adverse events and biomarkers:
- "Side effects" of e-cigarettes were recorded. Side effects were rated none, slight, mild, moderate and
severe at every assessment visit. An adverse effect possibly related to e-cigarette use was defined as
positive if the value at baseline was either none or slight AND the value at any of 3, 5, or 7 weeks was
mild or more severe

Other outcomes measured:

- Reduction in the average cpd

- E-cig adherence

- Nicotine withdrawal
Study funding
"MDS is a recipient of National Institute on Drug Abuse Award K24 DA000512. This award funded the project described here."

\begin{tabular}{ll}
\hline Author declarations & "None declared." \\
\hline Notes &
\end{tabular}

\section{Risk of bias}

\begin{tabular}{|c|c|c|}
\hline Bias & Authors' judgement & Support for judgement \\
\hline $\begin{array}{l}\text { Random sequence genera- } \\
\text { tion (selection bias) }\end{array}$ & High risk & No randomization \\
\hline $\begin{array}{l}\text { Allocation concealment } \\
\text { (selection bias) }\end{array}$ & High risk & No randomization \\
\hline $\begin{array}{l}\text { Incomplete outcome data } \\
\text { (attrition bias) } \\
\text { All outcomes }\end{array}$ & Low risk & $\begin{array}{l}\text { Quote: "One individual dropped out after week } 3 \text { and did not return; another } \\
\text { completed all follow-up assessments except week } 7 . "\end{array}$ \\
\hline $\begin{array}{l}\text { Selective reporting (re- } \\
\text { porting bias) }\end{array}$ & Low risk & All expected outcomes reported \\
\hline
\end{tabular}




\section{Study characteristics}

Methods

Design: Randomized, factorial trial (Participants were randomized to one of the 5 brands of e-cigarettes - although only 4 brands analyzed)

Recruitment: Media ads

Setting: Recruitment from the community, study took place at University, USA.

Study start date/Study end date: Not specified.

\section{Participants}

Total N: Analysis based on 24 (28 originally recruited, but the first 4 participants enrolled experienced malfunctioning NJOY e-cigs and withdrew - the project was removed from the market before the 5th participant was randomized)

N per arm: blu: 6; Green Smoke: 6; V2: 6; White Cloud: 6

Inclusion criteria:

- Age 18 to 65 and self-reported smoking at least 10 cigarettes per day.

Exclusion criteria:

- Use of other tobacco or nicotine-containing products, including e-cigarettes (no more than 3 previous episodes of use and not currently using)

- Current diagnosis or evidence of substance abuse or dependence or major depression

- Current or history of psychotic or bipolar disorder

- History of suicide attempt

- History of cancer or cardiovascular disease

- Uncontrolled hypertension

- Use of smoking cessation medications

- Any current plans to try to quit smoking

- Current pregnancy or lactation

Inclusion based on specific population characteristic: Not applicable

29\% women; mean age 43.3; mean cpd 17; mean FTND 3.7

Motivated to quit: Participants had no current plans to try to quit smoking (eligibility criterion)

E-cigarette use at baseline: No more than 3 previous episodes of use and not currently using (eligibility criterion)

\section{EC: Cig-a-like}

All participants received nicotine EC and were instructed to use them exclusively for 9 days

The 5 brands selected, including brand reported nicotine levels, were: (1) NJOY (18mg nicotine) - this brand was discontinued and not analyzed as the e-cigs provided malfunctioned; (2) V2, $18 \mathrm{mg}$ nicotine; (3) Green Smoke, 18.9 - 20.7 mg nicotine; (4) blu, 20 - 24mg nicotine; and (5) White Cloud, 23 - 24 $\mathrm{mg}$ nicotine. Each brand advertised the delivery of the same level of nicotine (appropriate for about a pack/day smoker), provided the standard tobacco flavor (no other flavors made available), and used a disposable cigarette-like device

Outcomes Day 10 is the only testing point of interest for us but participants were also tested at days 1 and 5 Adverse events and biomarkers:

- breath CO 
Strasser 2016 (Continued)

- direct effects of nicotine (e.g. dizzy, nauseas, headache) - visual analogue scale with a single word scored from 0 (not at all) to 100 (extremely). Total scores were summed such that higher scores indicated negative responses

Other outcomes measured:

- e-cigarette use

- direct effects of the e-cigarette (e.g. satisfying, calming, pleasant, smoke another right now) - visual analogue scale with a single word scored from 0 (not at all) to 100 (extremely). Total scores were summed such that higher scores indicated positive responses

- cotinine

- withdrawal and craving

Study funding

"National Cancer Institute $(\mathrm{NCl})$ of the National Institutes of Health $(\mathrm{NIH})$ and FDA Center for Tobacco Products (CTP) under Award Number P50CA179546, as well as grants from the National Cancer Institute (P50 CA143187, P30 CA16520, and P30 DA12393)"

Author declarations

"Dr Benowitz has served on scientific advisory boards for Pfizer and GlaxoSmithKline related to smoking cessation medications and has been an expert witness in litigation against tobacco companies. Dr Schnoll receives medication and placebo free of charge from Pfizer and has provided consultation to Pfizer and GlaxoSmithKline. These companies had no involvement in this study. Dr Strasser has received funding through the Pfizer GRAND program, an independent peer-reviewed grant program funded through Pfizer (2008-2011); all investigators have received funding from the United States National Institutes of Health"

Notes

\section{Risk of bias}

\begin{tabular}{|c|c|c|}
\hline Bias & Authors' judgement & Support for judgement \\
\hline $\begin{array}{l}\text { Random sequence genera- } \\
\text { tion (selection bias) }\end{array}$ & Unclear risk & $\begin{array}{l}\text { Although participants were randomized to different brands of EC, no descrip- } \\
\text { tion on how randomization was carried out }\end{array}$ \\
\hline $\begin{array}{l}\text { Allocation concealment } \\
\text { (selection bias) }\end{array}$ & Unclear risk & Not specified \\
\hline $\begin{array}{l}\text { Blinding of participants } \\
\text { and personnel (perfor- } \\
\text { mance bias) } \\
\text { All outcomes }\end{array}$ & Unclear risk & $\begin{array}{l}\text { No description of whether groups were blind to other conditions, but given } \\
\text { similar levels of support between arms, so performance bias judged unlikely }\end{array}$ \\
\hline $\begin{array}{l}\text { Blinding of outcome as- } \\
\text { sessment (detection bias) } \\
\text { All outcomes }\end{array}$ & Low risk & $\begin{array}{l}\text { Unclear whether any blinding took place, some outcomes were measured us- } \\
\text { ing objective measures and there was no difference in contact between arms }\end{array}$ \\
\hline $\begin{array}{l}\text { Incomplete outcome data } \\
\text { (attrition bias) } \\
\text { All outcomes }\end{array}$ & High risk & $\begin{array}{l}\text { For blu, Green Smoke, and V2 groups, } 83 \% \text { of participants completed the } 10 \text { - } \\
\text { day study; only } 33 \% \text { of participants randomized to White Cloud completed the } \\
\text { 10-day study; meaning loss to follow-up was considerably higher in this group }\end{array}$ \\
\hline $\begin{array}{l}\text { Selective reporting (re- } \\
\text { porting bias) }\end{array}$ & Low risk & All expected outcomes reported \\
\hline
\end{tabular}

\section{Study characteristics}


Tseng 2016 (Continued)

Methods
Design: 2-arm; double-blind placebo-controlled RCT

Recruitment: Advertisements placed in Craigslist as well as flyers distributed on the street and placed in New York City venues with details for how to contact study staff.

Setting: Community, USA

Study start date: July 2014 - 2015 (month unclear); Study end date: Not specified

Participants

Total N: 99 (100 were randomized but 1 participant randomized to the control arm was found to be ineligible between randomization and baseline)

N per arm: Nicotine EC: 50; Placebo EC: 49

Inclusion criteria:

- age 21 - 35 (confirmed with some form of identification document)

- daily smoker

- smoked $\geqq 10$ cigarettes a day (verified by a CO level of $\geq 8$ ppm)

- interested in reducing cigarette consumption

- able to provide consent

- had a cell phone and was willing/able to receive text messages and counseling on their cell phone

- willing to use an EC for 3 weeks

Exclusion criteria:

- Pregnant and/or breastfeeding

- had a history of asthma, other airways diseases, or heart disease

- were currently using smoking cessation medications (including other forms of NRT, bupropion, or varenicline), or enrolled in a smoking cessation program or another cessation trial.

- Use of EC in the past 14 days or any other tobacco products (pipe, cigar, cigarillos, snuff, chewing tobacco, rolling tobacco, or hookah/shisha) in the past 30 days

- having a moderate to severe drug use disorder defined as a score of at least 5 on the Drug Abuse Screening Test- 10 and/or a hazardous or active alcohol use disorder defined as at least 7 for men and at least 5 for women on the Alcohol Use Disorders Identification

Inclusion based on specific population characteristic: Young adults

32.3\% women; mean age 28.43; mean cpd 14.33; FTND not measured but time to first cigarette was measured categorically. The mode category was 6 - 30 mins (39/99; 41.5\%) Smoking behavioral dependence scale (11 items): mode category 'Moderate' (51/99; 51.5\%)

Motivated to quit: Readiness to quit ( $1-10$ scale, $1-8$ apply to current people who smoke): $5.57 \pm 1.49$

E-cigarette use at baseline: No use of e-cigs in past 14 days (eligibility criterion)
Interventions

\section{EC: Cig-a-like}

E-cigarette details:

3 weeks of disposable 4.5\% nicotine NJOY, King Bold (NJOY, Inc, Scottsdale, AZ) which resemble conventional cigarettes. NJOY also manufactured the non-nicotine placebo EC. Both nicotine and placebo ECs were tobacco-flavored. The products were purchased by the investigators and provided to the participants free of charge

Other stop-smoking pharmacotherapies: None

Behavioural support:

Prior to receiving the ECs, participants were required to complete a 20- to 30-minute telephone counseling session with a trained tobacco cessation Counsellor. The purpose of the telephone counseling was to review current smoking patterns and offer behavioral and environmental change strategies. 
These included specific smoking reduction options, such as eliminating cigarettes at work and in the home, carrying only those cigarettes needed for that day, dropping cigarettes associated with less intense triggers first, avoiding smoking triggers, and other strategies to manage urges.18 participants were asked to reduce the number of cigarettes smoked daily by at least $50 \%$ of the total number of cigarettes smoked per day at baseline. To mimic real-life EC use, minimum EC use instruction was provided. Participants were encouraged to replace cigarettes with as much or as little use of an EC as needed in order to reduce nicotine withdrawal symptoms

Week 1,3
Cessation: Not applicable
Adverse events and biomarkers: adverse events and symptoms
Other outcomes measured:
- self-reported reduction of at least $50 \%$ in the number of cpd
- percentage reduction in number of cpd
- Use of ECs
- satisfaction with ECs

Study funding

"This work was supported by the National Center for Advancing Translational Sciences at the National Institutes of Health (grant number UL1TR000038)."

\begin{tabular}{ll} 
Author declarations & "None declared" \\
\hline Notes & Study listed as ongoing study NCT02628964 in the 2016 review update
\end{tabular}

\section{Risk of bias}

\begin{tabular}{lll}
\hline Bias & Authors' judgement & Support for judgement \\
\hline $\begin{array}{l}\text { Random sequence genera- } \\
\text { tion (selection bias) }\end{array}$ & Low risk & Quote: "computer generated” \\
\hline $\begin{array}{l}\text { Allocation concealment } \\
\text { (selection bias) }\end{array}$ & Unclear risk & $\begin{array}{l}\text { Quote: “...was concealed from research assistants. Blinding of the allocation } \\
\text { of nicotine or placebo EC was ensured by the identical appearance of the ECs". } \\
\text { However, not enough information given on how allocation was concealed at } \\
\text { the point of randomization }\end{array}$ \\
\hline
\end{tabular}

$\begin{array}{ll}\begin{array}{l}\text { Blinding of participants } \\ \text { and personnel (perfor- }\end{array} & \text { Low risk }\end{array}$
mance bias)

All outcomes

\begin{tabular}{|c|c|c|}
\hline $\begin{array}{l}\text { Blinding of outcome as- } \\
\text { sessment (detection bias) }\end{array}$ & Low risk & $\begin{array}{l}\text { Quote: "Blinding of the allocation of nicotine or placebo EC was ensured by the } \\
\text { identical appearance of the ECs" }\end{array}$ \\
\hline
\end{tabular}

All outcomes

Incomplete outcome data Low risk Nicotine EC Itfu: 10/50; Placebo EC Itfu: 10/49
(attrition bias)

All outcomes

Selective reporting (re- Low risk $\quad$ All expected outcomes reported
porting bias)


Valentine 2018

\section{Study characteristics}

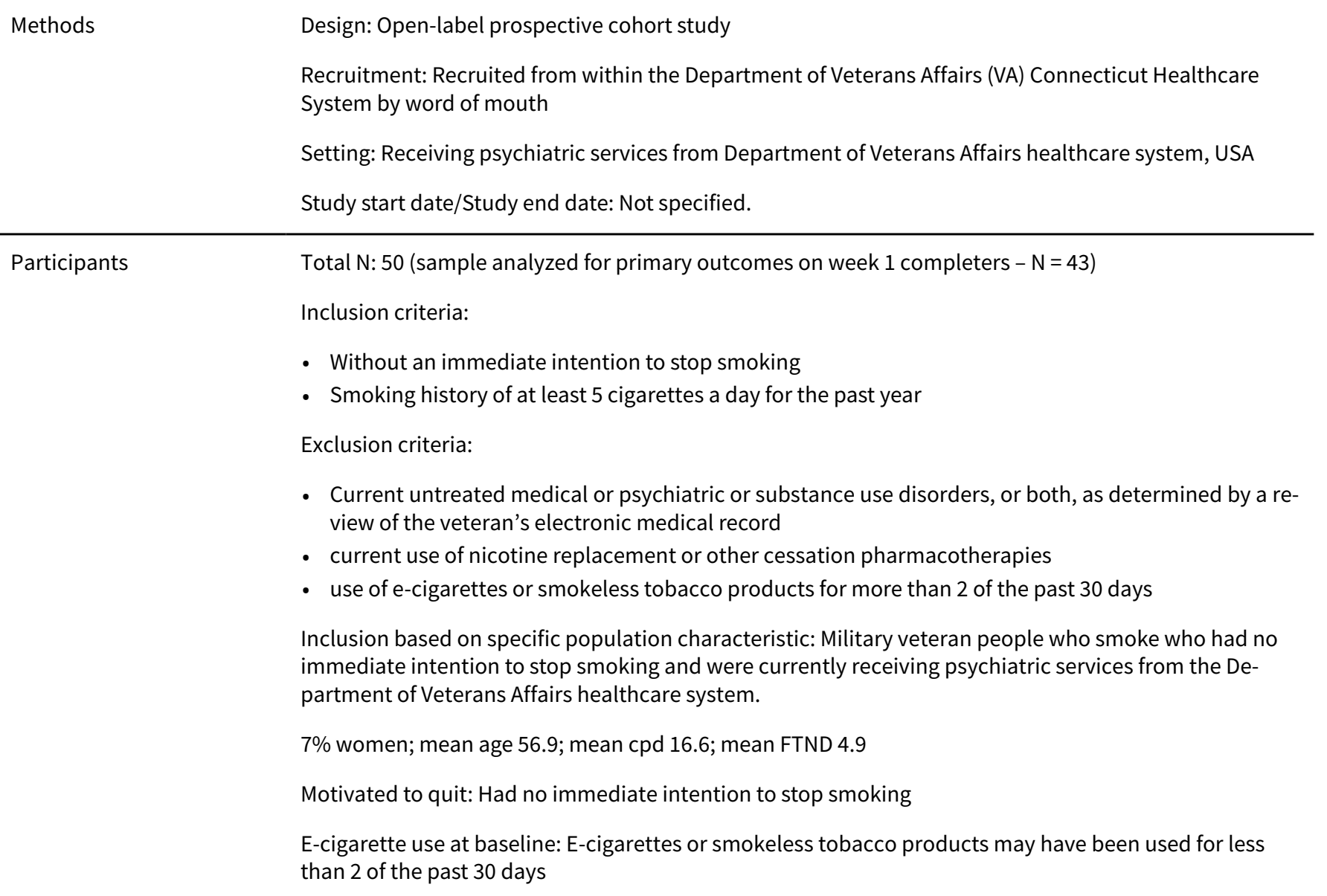

Interventions

\section{EC: Refillable}

All given eVic Supreme (Joyetech), "a commercial, variable-power, tank-type device". $6.5 \mathrm{~mL}$ tank (Delta 23, Joyetech) and a C3 triple coil atomizer head (Joyetech) with a total resistance of 1.8 ohms. Participants could choose flavor (menthol or tobacco) and nicotine concentration (12 or $24 \mathrm{mg} / \mathrm{mL}$ ).

Participants taught how to use EC, with additional materials dispensed as needed. Participants were informed that they could use the study e-cigarette or regular tobacco cigarettes, or both, ad libitum during study participation

Outcomes

Week 1, 2, 3, 4, 8 (Weekly lab visits and 1 month follow-up)

Adverse events and biomarkers: Alveolar (breath) CO levels (ppm)

Other outcomes measured:

- Number of cpd

- The frequency of e-cigarette use (mean days/week)

- The amount of money spent on combustible cigarettes (US dollars/week)

- Fagerström Test of Nicotine Dependence

- Contemplation Ladder

- E-cigarette questionnaire (assessed changes in perceptions about e-cigarettes (e.g. harmfulness, benefits, cost), motivations to use (or not use) them, and the reasons for e-cigarette or combustible cigarette preferences) (measured at baseline and follow-up)

- Cotinine 
Valentine 2018 (Continued)

Study funding
"This research was supported by the New England Mental Illness Research, Education and Clinical Center and the U.S. Department of Veterans Affairs. Statistical analyses, biochemical assays, and analyses of e-cigarette solutions were supported by the Administrative and Laboratory cores of P50DA036151 (Yale TCORS) from the National Institutes of Health and the U.S. Food and Drug Administration Center for Tobacco Products. The content is solely the responsibility of the authors and does not necessarily represent the official views of the National Institutes of Health or of the U.S. Food and Drug Administration."

\section{Author declarations}

"Ralitza Gueorguivea, PhD, discloses consulting fees for Palo Alto Health Sciences and Mathematica Policy Research and a provisional patent submission by Yale University: Chekroud, A. M., Gueorguieva, R., \& Krystal, K. H. "Treatment Selection for Major Depressive Disorder" (filing date June 3, 2016, USPTO docket number Y0087.70116US00). The authors report no other financial relationships with commercial interests."

Notes New for 2020 update.

\section{Risk of bias}

\begin{tabular}{|c|c|c|}
\hline Bias & Authors' judgement & Support for judgement \\
\hline $\begin{array}{l}\text { Random sequence genera- } \\
\text { tion (selection bias) }\end{array}$ & High risk & Uncontrolled cohort study \\
\hline $\begin{array}{l}\text { Allocation concealment } \\
\text { (selection bias) }\end{array}$ & High risk & Uncontrolled cohort study \\
\hline $\begin{array}{l}\text { Incomplete outcome data } \\
\text { (attrition bias) } \\
\text { All outcomes }\end{array}$ & Unclear risk & Follow-up: $31 / 50$ at week 8 \\
\hline $\begin{array}{l}\text { Selective reporting (re- } \\
\text { porting bias) }\end{array}$ & Unclear risk & No protocol or clinical trial record. \\
\hline
\end{tabular}

\section{Van Staden 2013}

\section{Study characteristics}

$\begin{array}{ll}\text { Methods } & \text { Design: Single-group within-subject design } \\ \text { Recruitment: Participants from a military hospital in South Africa } \\ \text { Setting: South Africa } \\ \text { Study start date/ end date: Not specified } \\ \text { Total N: } 15 \text {, mean age } 38 \text { years, smoked } 20 \text { cpd (range } 10 \text { - 30), for an average of } 17 \text { years (range } 5 \text { - 27) } \\ \text { Total N: } 13 \text { completed the study (5 women) } \\ \text { Inclusion criteria: } \\ \text { - Adults who smoke daily, of at least } 10 \text { cpd } \\ \text { Exclusion criteria: } \\ \text { - History of lung disease } \\ \text { Inclusion based on specific population characteristic: No }\end{array}$


Van Staden 2013 (Continued)

Motivated to quit: Not specified

E-cigarette use at baseline: Not specified

EC: Cig-a-like
Participants were asked to use an EC only for 2 weeks (i.e. no cigarettes)
EC: 'Twisp eGo' cartridge $0.8 \mathrm{ml}$ containing $0.0144 \mathrm{mg}$ of nicotine

Outcomes

The following measurements were taken at baseline and 2-week follow-up:

- Blood pressure and pulse

- Arterial and venous $\mathrm{COHb}$ and blood oxygen saturation

Study funding

"We are grateful for the sponsorship of the eGo e-cigarette packs by Twisp and also for the valuable advice and laboratory assistance given by Col. (Dr) J Lubbe, Chemical Pathologist, 1 Military Hospital, Pretoria with regard to the measurement of the cotinine levels. We also wish to acknowledge Professor Martin Veller for his insightful contributions during the preparation of this manuscript and also $\mathrm{Dr}$ Richard van Zyl-Smith for his assistance and review."

\begin{tabular}{|c|c|c|}
\hline Author declarations & \multicolumn{2}{|c|}{$\begin{array}{l}\text { "The sponsor of the Twisp e-cigarette had no role in the design and conduction; the collection, analysis } \\
\text { and interpretation of the study; or in the preparation, review or approval of the manuscript." }\end{array}$} \\
\hline Notes & \multicolumn{2}{|c|}{$\begin{array}{l}\text { Dropouts ( } N=2 \text { ) were due to illness (headache and fever) and undertaking a military course associated } \\
\text { with high stress and exposure to others smoking, making it difficult to abstain from cigarettes }\end{array}$} \\
\hline & \multicolumn{2}{|c|}{$\begin{array}{l}\text { The paper states that the EC cartridge contained } 0.8 \mathrm{ml} \text { of solution with } 0.0144 \mathrm{mg} \text { of nicotine. This } \\
\text { would be an unusually low concentration of nicotine and we have assumed an error in units where mil- } \\
\text { ligrams should have been grams ( } 0.0144 \text { grams of nicotine would make the concentration } 18 \mathrm{mg} / \mathrm{ml} \text { ) }\end{array}$} \\
\hline \multicolumn{3}{|l|}{ Risk of bias } \\
\hline Bias & Authors' judgement & Support for judgement \\
\hline $\begin{array}{l}\text { Random sequence genera- } \\
\text { tion (selection bias) }\end{array}$ & High risk & Prospective cohort \\
\hline $\begin{array}{l}\text { Allocation concealment } \\
\text { (selection bias) }\end{array}$ & High risk & Not randomized \\
\hline $\begin{array}{l}\text { Incomplete outcome data } \\
\text { (attrition bias) } \\
\text { All outcomes }\end{array}$ & Low risk & $2 / 15$ lost to follow-up \\
\hline $\begin{array}{l}\text { Selective reporting (re- } \\
\text { porting bias) }\end{array}$ & Unclear risk & Unable to determine prespecified outcomes \\
\hline
\end{tabular}

Veldheer 2019

\section{Study characteristics}

Design: Randomized parallel-assignment double-blind trial
Setting: USA ( 2 sites)
Recruitment: Community advertisements


Total N: 263 in this analysis (520 planned overall; THIS INCLUDES ONLY THOSE FOLLOWED UP AT 1 AND 3 MONTHS)

N per arm: sub: 72; EC: 191

Inclusion criteria:

- Age $21-65$

- Smoke > 9 cigarettes per day for at least 1 year

- Smoke regular filtered cigarettes or machine-rolled cigarettes with a filter

- CO measurement > 9 ppm at baseline

- No serious quit attempt in the prior 1 month. This includes use of any FDA-approved smoking cessation medication (varenicline, bupropion (used specifically as a quitting aid), patch, gum, lozenge, inhaler, and nasal spray) in the past 1 month as an indication of treatment-seeking

- Not planning to quit in the next 6 months

- Interested in reducing cigarette consumption

- Willing to attend visits weekly and monthly over a 9-month period (not planning to move, not planning extended vacation, no planned surgeries)

- Read and write in English

- Able to understand and consent

Exclusion criteria:

- Pregnant and/or nursing women

- Unstable or significant medical condition in the past 12 months (recent heart attack or some other heart conditions, stroke, severe angina including high blood pressure if systolic $>159$ or diastolic $>99$ observed during screening)

- Immune system disorders, respiratory diseases (exacerbations of asthma or COPD, require oxygen, require oral prednisone), kidney (dialysis) or liver diseases (cirrhosis), or any medical disorder/medication that may affect participant safety or biomarker data

- Use of any non-cigarette nicotine delivery product (pipe, cigar, dip, chew, snus, hookah, e-cigs, strips, sticks) in the past 7 days

- Uncontrolled mental illness or substance abuse or inpatient treatment for these in the past 6 months

- History of difficulty providing or unwilling to provide blood samples (fainting, poor veins, anxiety)

- No surgery requiring general anesthesia in the past 6 weeks

- Use of an e-cig for 5 or more days in the past 28 days or any use in the past 7 days

- Use of marijuana or any illicit drug/prescription drugs for non-medical use daily/almost daily, or weekly in the past 3 months per NIDA Quick Screen

- Use of hand-rolled, roll-your-own cigarettes

- Known allergy to propylene glycol or vegetable glycerin

- Other member of household is currently participating/participated in the study

$58 \%$ women; mean age 47; mean cpd 18; mean FTND: Not specified

Motivated to quit: Interested in reducing cigarette intake but not planning to quit in next 6 months

E-cigarette use at baseline: None

Interventions

\section{EC: Cig-a-like}

For 24 weeks:

1) Cigarette substitute: QuitSmart cigarette substitute - plastic tube looks like a real cigarette, designed to provide the same draw resistance as a smoker's usual cigarette. No drug delivery. 2 cigarette substitutes and a product manual are provided to participants following randomization and replace- 
ment products are provided throughout the intervention period ( 24 weeks). At baseline, associated user manual, research staff explain how to use product. Reduction goal to $50 \%$ at weeks 0 and $1,75 \%$ at weeks 2 and 4 , continue reducing onwards from there

2) EC with no nicotine: $E G O$ e-cigarette. Cartomizers containing $0 \mathrm{mg} / \mathrm{ml}$ nicotine provided throughout the intervention period ( 24 weeks) Associated user manual, research staff explain how to use product.

3) As (2) but $8 \mathrm{mg} / \mathrm{ml}$ nicotine

4) As (2) but $36 \mathrm{mg} / \mathrm{ml}$ nicotine

Months 1, 3, 6, 9; (only 1 and 3 month available at time of extraction)
Cessation: Conventional tobacco product use measured but measures not clear
Adverse events and biomarkers:
- Adverse events
- Lung function
- Blood pressure, pulse
- CO, "exhaled breath condensate biomarkers of oxidative stress, glutathione and 8 Isoprostanes" - in-
cl. carcinogenic nitrosamine 4-(methylnitrosamino)-1-(3-pyridyl)-1-butanone [NNK; via its metabolite
NNAL (4-(methylnitrosamino)-1-(3-pyridyl)-1-butanol) in urine], expired air carbon monoxide (CO),
and nicotine (via its metabolite cotinine in urine)

Other outcomes measured:

- Weight

- Cotinine

- Tobacco use

Study funding

This study was funded by the National Institutes of Health (NIH) and the U.S. Food and Drug Administration (FDA) under Award Number P50DA036105. The content is solely the responsibility of the authors and does not necessarily represent the views of the NIH or FDA. The project [publication] was supported by CTSA award No. UL1TR000058 from the National Center for Advancing Translational Sciences. Its contents are solely the responsibility of the authors and do not necessarily represent official views of the National Center for Advancing Translational Sciences or the National Institutes of Health.

Author declarations

JF has done paid consulting for pharmaceutical companies involved in producing smoking cessation medications, including GSK, Pfizer, Novartis, J\&J, and Cypress Bioscience. TE is a paid consultant in litigation against the tobacco industry and is named on a patent application for a device that measures the puffing behavior of electronic cigarette users. There are no competing interests to declare for other authors

Notes

Preliminary data from RCT; full results not yet available

EC arms pooled in preliminary data available to us at time of writing

Authors provided outcome data; Study listed as ongoing study Lopez 2016 in the 2016 review update

\section{Risk of bias}

\begin{tabular}{lll}
\hline Bias & Authors' judgement & Support for judgement \\
\hline $\begin{array}{l}\text { Random sequence genera- } \\
\text { tion (selection bias) }\end{array}$ & Low risk & $\begin{array}{l}\text { Quote: "the assignment codes are made from separate randomization lists cre- } \\
\text { ated in advance by the statistician for each site stratum." }\end{array}$ \\
\hline $\begin{array}{l}\text { Allocation concealment } \\
\text { (selection bias) }\end{array}$ & Low risk & $\begin{array}{l}\text { Quote: "Once a participant has been confirmed eligible for randomization, a } \\
\text { computer procedure will assign the participant to the next condition on the list } \\
\text { automatically." }\end{array}$ \\
\hline
\end{tabular}


Veldheer 2019 (Continued)

Blinding of participants Low risk Not blinded for non-EC arms but given similar level of support/product, so perand personnel (perforformance bias judged unlikely mance bias)

All outcomes

$\begin{array}{ll}\begin{array}{l}\text { Blinding of outcome as- } \\ \text { sessment (detection bias) }\end{array} & \text { Low risk }\end{array} \quad \begin{aligned} & \text { Not blinded for non-EC arms but given similar level of support/product, so dif- } \\ & \text { ferential misreport judged unlikely }\end{aligned}$

All outcomes

\begin{tabular}{lll}
\hline $\begin{array}{l}\text { Incomplete outcome data } \\
\text { (attrition bias) } \\
\text { All outcomes }\end{array}$ & Unclear risk & $\begin{array}{l}\text { Dataset only includes those followed up at } 1 \text { and } 3 \text { months, which excludes } \\
140 \text { participants; breakdown by arm not provided }\end{array}$ \\
\hline $\begin{array}{l}\text { Selective reporting (re- } \\
\text { porting bias) }\end{array}$ & High risk & $\begin{array}{l}\text { Results paper just preliminary results with all EC arms collapsed. Protocol and } \\
\text { NCT record list different outcomes and study lengths. }\end{array}$ \\
\hline
\end{tabular}

Wadia 2016

\section{Study characteristics}

\begin{tabular}{|c|c|}
\hline Methods & $\begin{array}{l}\text { Design: Uncontrolled experimental study } \\
\text { Recruitment: Dental hospital staff were recruited - not specified how } \\
\text { Setting: Dental hospital, UK } \\
\text { Study start date: April 2015; Study end date: December } 2015\end{array}$ \\
\hline Participants & $\begin{array}{l}\text { Total N: } 20 \text { ( } 18 \text { of the } 20 \text { attended the reassessment visit) } \\
\text { Inclusion criteria: } \\
\text { - } 18 \text { - } 65 \text { years old } \\
\text { - Systemically healthy } \\
\text { - Smoked at least } 10 \text { cigarettes per day for at least } 5 \text { years } \\
\text { - had at least } 24 \text { natural teeth (excluding third molars) and had no probing pocket depths over } 4 \text { mm } \\
\text { at any site } \\
\text { - did not wish to quit } \\
\text { Exclusion criteria: } \\
\text { - Participants were excluded if they had a systemic condition known to exacerbate or modulate peri- } \\
\text { odontitis (for example, diabetes) } \\
\text { - antibiotics had been taken in the previous } 3 \text { months } \\
\text { - anti-inflammatory drugs or other medication likely to affect the periodontal tissues were taken rou- } \\
\text { tinely } \\
\text { - if they were pregnant or a nursing mother } \\
\text { \% women, age, cpd and FTND: not specified. } \\
\text { Motivated to quit: enrolled people who smoke who did not intend to quit smoking, but were prepared } \\
\text { to attempt to substitute smoking with the use of e-cigarettes for } 2 \text { weeks } \\
\text { E-cigarette use at baseline: not specified }\end{array}$ \\
\hline
\end{tabular}


Wadia 2016 (Continued)

Participants provided with a blu PROTM e-cigarette kit (Electric Tobacconist ${ }^{\circledR}$ ), an extra bottle of blu PRO Tobacco ${ }^{\mathrm{TM}}$ e-Liquid (Electric Tobacconist) and written instructions. The e-Liquid was Classic Tobacco-flavoured and contained $18 \mathrm{mg}$ of nicotine (medium strength). The participants agreed to substitute their regular smoking habits with the use of e-cigarettes for 2 weeks. They were asked to make a note of any cigarette smoking during the 2 weeks if complete abstinence was unsuccessful

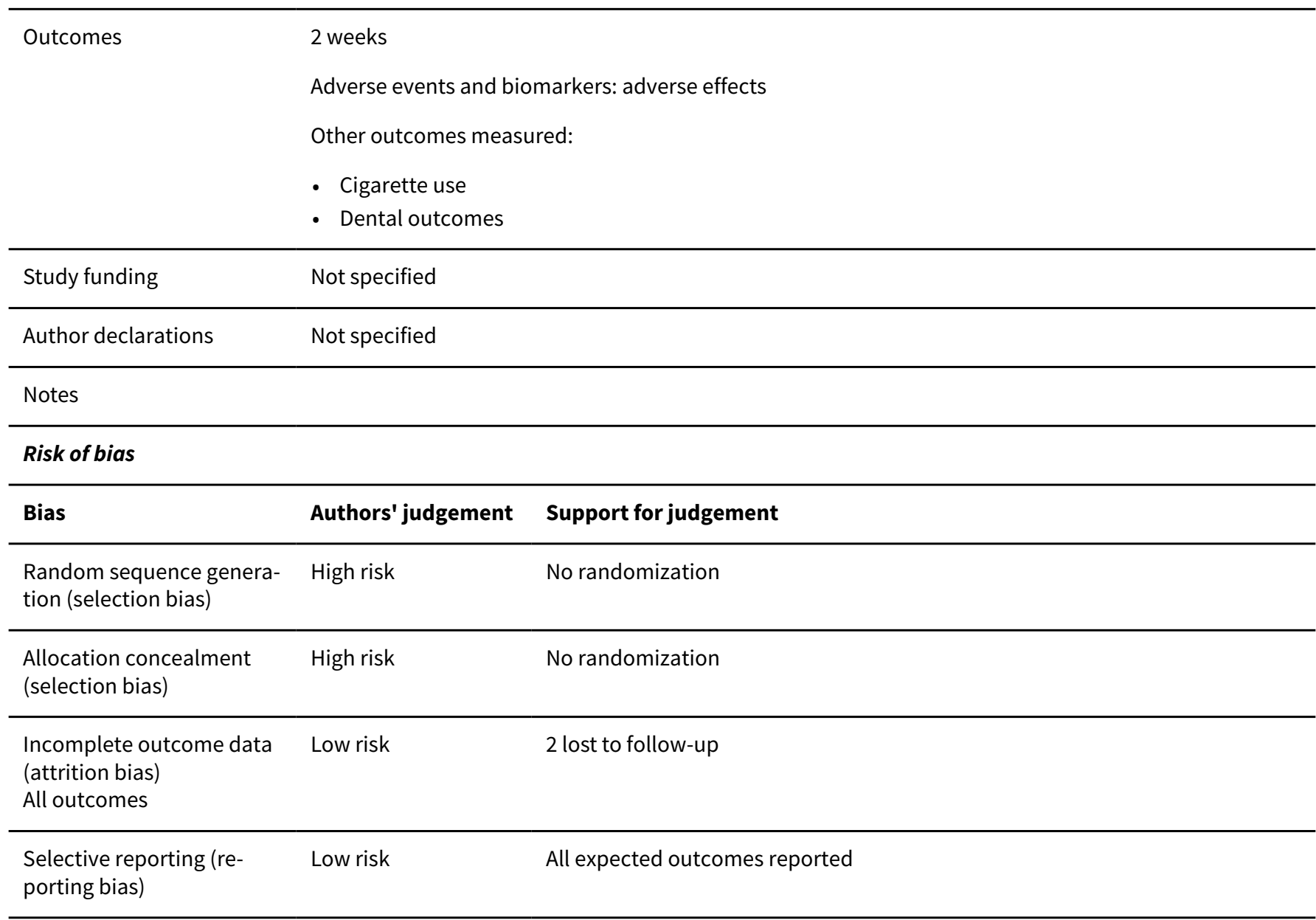

Walele 2018

\section{Study characteristics}

Methods Design: RCT (short-term, Cravo 2016) followed by cohort study (Walele 2018) in which all participants were given nicotine $\mathrm{EC}$

Recruitment: Community

Setting: 2 centres in the UK (Covance Clinical Research Unit Ltd, Leeds and Simbec Research Ltd, Wales)

Study start date: December 2013; Study end date: December 2016

$\begin{array}{ll}\text { Participants } & 420 \text { participants } \\ & \text { Inclusion criteria differ per study phase: }\end{array}$

\section{Cravo 2016 (short-term RCT):}

- 21 - 65 years of age 
- BMI $18-35 \mathrm{~kg} / \mathrm{m}^{2}$

- 5 - 30 cigarettes per day for at least 1 year (self-reported)

- in good health (determined by medical history, a physical examination, a 12-lead ECG, lung function tests and clinical laboratory evaluations)

- Established people who smoke (urinary cotinine $\geq 3$ and exhaled $\mathrm{CO} \geq 6 \mathrm{ppm}$ )

\section{Additional criteria for Walele 2018 (participants from Cravo 2016):}

- Participants assessed by PI as being compliant in Cravo 2016 (e.g. having attended outpatient visits and having been compliant with study procedures)

- Participants had to be willing to use the study product as the only nicotine-containing product for the duration of the study, and, as deemed by PI, had to have no clinically significant abnormalities in 12lead electrocardiogram, vital signs, spirometry and clinical laboratory assessments in the preceding study

- In addition, participants who were assigned to the conventional cigarette (CC arm) in Cravo 2016 had to be established people who smoke CCs, which was assessed by urinary cotinine levels (a score of 3 and above on a NicAlert ${ }^{\mathrm{TM}}$ test strip was considered positive), eCO levels (a readout $>6 \mathrm{ppm}$ was considered positive) and by review of a smoking history questionnaire

Exclusion criteria:

\section{Cravo 2016:}

- Use of NRT, snuff or chewing tobacco in 14 days previous, or intended to use during study

- Trying to stop smoking or considering quitting

- Clinically-significant illness or disorder, history of drug or alcohol abuse within 2 years prior to study start

- Woman of "childbearing potential" unwilling to use "acceptable contraceptive measure" during study

Walele 2018 (participants from Cravo 2016):

- People who had taken or received any form of NRT, snuff or chewing tobacco during the previous study or intended to use it during this study, were excluded

- People with relevant illness history

- People with history of drug or alcohol abuse

- People with lung function test or vital signs considered unsuitable

- People who are trying to stop smoking

- Women who are pregnant, or unwilling to use acceptable contraceptive method for the duration of the study

\section{Cravo 2016}

Total N: 419 randomized, 408 analyzed (excludes 11 who were excluded prior to any product use)

N per arm: EVP: 306; Control: 102

$45 \%$ women; mean age 34.6; Mean cpd: most 11 - 20 cpd (56\% int, $62 \%$ control); Mean FTND: most moderate (57\% int, $54 \%$ cont)

Motivated to quit: No

E-cigarette use at baseline: Not excluded based on prior EC use

Walele 2018

Total N: 209 (147 pre-EVP group; 62 pre-CC group)

45\% women; mean age 36.6; mean cpd 2.6 (data from figure): Not reported; FTND: Not reported

Motivated to quit: As reported for Cravo 2016 
Walele 2018 (Continued)

E-cigarette use at baseline: Not reported
Interventions

\section{EC: Cig-a-like}

\section{Cravo 2016}

EC: EVP prototype (2.0\% nicotine), developed by Fontem Ventures B.V. (Amsterdam, the Netherlands). Instructed to only use EVP for study period. It consisted of a rechargeable battery (voltage range of $3.0 \mathrm{e} 4.2 \mathrm{~V}$ ), an atomiser and a capsule (small cartridge) containing e-liquid. The capsules were replaceable and the battery and atomiser were reusable. Could choose between two different e-liquids, which differed solely in their flavor: a menthol-flavored e-liquid with $2.0 \%$ nicotine ( $2.7 \mathrm{mg}$ nicotine/capsule) and a tobacco-flavored e-liquid with $2.0 \%$ nicotine (2.7 $\mathrm{mg}$ nicotine/capsule)

Control: Used their own usual conventional cigarette brand

\section{Walele 2018}

E-cigarette details: Commercially available Puritane ${ }^{\mathrm{TM}}$ (closed system EVP) consists of a lithium-ion rechargeable battery and a replaceable cartomiser comprising of an e-liquid reservoir pre-filled by the manufacturer, a heating element and a mouthpiece; $1.6 \%$ nicotine $(16 \mathrm{mg} / \mathrm{g})$ Available in tobacco or menthol. 2 weeks before baseline, participants had a familiarization session with Puritane ${ }^{\mathrm{TM}}$, where they could see and try the EVP

Outcomes

Cravo 2016: Weeks 1, 2, 4, 6, 8, 10 and 12

Walele 2018: starting on the last day of the previous trial): Months 1, 2, 3, 6, 9, 12, 15, 18, 21 and 24

Study centre visits for assessments

Adverse events and biomarkers:

- "adverse events" (coded using Medical Dictionary for Regulatory Activities version 16.1, 2013, collected via diary cards and questionnaires)

- vital signs (systolic and diastolic blood pressure, pulse rate and oral temperature)

- lung function (FEV, FEF, PEF, FEV)

- urine biomarkers (nicotine equivalents (NEQs: nicotine, cotinine, nicotine-N-glucuronide, cotinine-Nglucuronide, trans 3'-hydroxycotinine and trans 3'-hydroxycotinine glucuronide); S-PMA; 3-HPMA; PG; total NNAL (NNAL p NNAL-glucuronide)); exhaled CO

- blood $\mathrm{COHb}$

Other outcomes measured:

- Number of conventional cigarettes smoked

- EVP capsules used

- ECG (categorized them as normal, abnormal-not clinically significant (NCS) or abnormal-clinically significant (CS))

- MWS-R (revised Minnesota Nicotine Withdrawal Scale)

- QSUBrief (Brief Questionnaire of Smoking Urges) questionnaires

- clinical chemistry (blood levels of aspartate aminotransferase (AST), alanine aminotransferase (ALT), alkaline phosphatase, gamma-glutamyl transferase (GGT), sodium, potassium, chloride, calcium, inorganic phosphate, glucose, urea nitrogen (BUN), total bilirubin, creatinine, total protein, albumin, cholesterol (HDL, LDL, and total)); clinical haematology (white blood cell count (WBC), red blood cell count (RBC), hemoglobin, hematocrit (PCV), mean cell volume (MCV), mean cell hemoglobin ( $M C H)$, mean cell hemoglobin concentration (MCHC), platelet count, differential $\mathrm{WBC}$ ); urinalysis ( $\mathrm{pH}$, protein, glucose, ketones, urobilinogen, blood and specific gravity)

Study funding

"This work was funded and supported by Fontem Ventures B.V. Imperial Brands plc is the parent company of Fontem Ventures B.V. the 
Walele 2018 (Continued)

manufacturer of the EVP prototype used in this study"

\section{Walele 2018}

"This work was funded and supported by Fontem Ventures B.V. Imperial Brands Group plc is the parent company of Fontem Ventures B.V., the

manufacturer of the EVP used in this study"

Author declarations

\section{Cravo 2016}

"Dr. Cravo has nothing to disclose. Mrs Martin reports personal fees from Fontem Ventures B.V. during the conduct of the study; personal fees from Tobacco and pharmaceutical industries outside the submitted work. Dr. Sharma reports other from Fontem Ventures B.V. during the conduct of the study. Dr. Bush reports other from Fontem Ventures B.V. during the conduct of the study. Mrs Savioz reports personal fees from Fontem Ventures B.V. during the conduct of the study; personal fees from Tobacco and pharmaceutical industries outside the submitted work. Mr Craige has nothing to disclose. Mr Walele has nothing to disclose."

Walele 2018 (copied from Transparency documents)

"Dr. Koch reports other from Fontem Ventures B.V., during the conduct of the study; Dr. Martin reports personal fees from Fontem Ventures B.V., during the conduct of the study; personal fees from Tobacco and pharmaceutical industries, outside the submitted work; Dr. O'Connell has nothing to disclose. Dr. Bush reports other from Fontem Ventures B.V., during the conduct of the study; Dr. Savioz reports personal fees from Fontem Ventures B.V., during the conduct of the study; personal fees from Tobacco and pharmaceutical industries, outside the submitted work; Dr. Walele has nothing to disclose."
Sponsor: Imperial Tobacco Group PLC

Study listed as ongoing studies NCT02029196 and NCT02143310 in 2016 review update. Treated as single study in this review due to including

the same participants, and no time lag between studies

"The same subjects who participated in our previous clinical trial (ClinicalTrials.gov, \#NCT02029196) conducted in the same centres, with another EVP (Cravo et al., 2016), were invited to participate the study by Walele 2018. All volunteering subjects were assigned to switch to using Puritane ${ }^{\mathrm{TM}}$, a closed system EVP, for two years, starting on the last day of the previous trial (End of Study [EoS] visit), which corresponded to the baseline visit of Walele 2018."

\section{Risk of bias}

\begin{tabular}{|c|c|c|}
\hline Bias & Authors' judgement & Support for judgement \\
\hline $\begin{array}{l}\text { Random sequence genera- } \\
\text { tion (selection bias) }\end{array}$ & Low risk & $\begin{array}{l}\text { Quote: "Randomisation was performed using an Interactive Web Response } \\
\text { System (IWRS; Almac Clinical Technologies)" }\end{array}$ \\
\hline $\begin{array}{l}\text { Allocation concealment } \\
\text { (selection bias) }\end{array}$ & Low risk & $\begin{array}{l}\text { Quote: "Randomisation was performed using an Interactive Web Response } \\
\text { System (IWRS; Almac Clinical Technologies)" }\end{array}$ \\
\hline $\begin{array}{l}\text { Blinding of participants } \\
\text { and personnel (perfor- } \\
\text { mance bias) } \\
\text { All outcomes }\end{array}$ & High risk & $\begin{array}{l}\text { Open-label, no blinding, differential levels of support/product use so perfor- } \\
\text { mance bias cannot be ruled out }\end{array}$ \\
\hline $\begin{array}{l}\text { Blinding of outcome as- } \\
\text { sessment (detection bias) } \\
\text { All outcomes }\end{array}$ & High risk & $\begin{array}{l}\text { Open-label, no blinding, with differential levels of support/product use and } \\
\text { subjective outcomes }\end{array}$ \\
\hline
\end{tabular}


Walele 2018 (Continued)

Incomplete outcome data Low risk (attrition bias)

All outcomes
Cravo: $286 / 306$ int (4.5\% ltfp) and 101/102 (1\% ltfp) control completed study but all who received product included in analysis. In EVP group, 14 withdrew consent, 2 experienced AEs, 1 death, 3 "other". CC group $1 \mathrm{AE}$

Walele 2018: High

209/387 enrolled for study Walele 2018. A total of 102 participants (48.8\%; EVP: 75/145 (51\%); CC: $27 / 61$ (43.5\%) completed the study

Selective reporting (re- Low risk
porting bias)

\section{Cravo 2016: Low}

All anticipated outcomes reported (study registered prior to study completion)

Walele 2018: Low

All anticipated outcomes reported (study registered prior to study completion)

Walker 2020

\section{Study characteristics}

\begin{tabular}{ll}
\hline Methods & Design: RCT \\
& Recruitment: National media advertising \\
& Setting: Community based, New Zealand \\
& Study start date: Recruitment between March 2016; Study end date: Aug 2018 \\
\hline
\end{tabular}

Participants

N per arm: Patches-only group: 125; Patches plus nicotine e-cigarette group: 500; Patches plus nicotine-free e-cigarette group: 499

Inclusion criteria:

- Eligible if they were living in New Zealand

- 18 years or older

- smoked tobacco (amount not specified)

- Motivated to quit in the next 2 weeks

- Able to provide verbal consent

- Prepared to use any of the trial treatments

- Had access to a telephone

Exclusion criteria:

- Pregnant or breastfeeding women

- Had used an e-cigarette for smoking cessation for more than 1 week anytime in the past year

- Currently using smoking cessation medication

- Enrolled in another cessation program or study

- Self-reported a history of severe allergies

- Poorly-controlled asthma

- Cardiovascular event in the 2 weeks before enrolment

- Only 1 participant per household was permitted.

69\% women; mean age 41.6; mean cpd 17.3; mean FTND 5.2

Motivated to quit: yes 
Interventions

\section{EC: Refillable}

Moderate-intensity behavioral support was available for all participants immediately after randomization, then once a week for 6 weeks. This support consisted of $10-15$ mins of withdrawal-oriented behavioral support and advice on using their allocated treatment, delivered proactively over the phone by researchers who had received standardized training in delivery of such support. Assigned to:

1) Nicotine patch for 14 weeks including 2 week prequit. $21 \mathrm{mg}$, 24-hr nicotine patch (Habitrol)

2) Nicotine patch and nicotine-free EC for 14 weeks. As 1, plus 14-week supply at no cost. A 2nd generation eVOD (Kangertech, Shenzhen GuangDong, China) starter kit, with a choice of 1 of 2 tobacco e-liquid flavors. Advised to start using the e-cigarette 2 weeks before their quit date, as and when necessary or desired, and in accordance with the manufacturer's written instructions, to become familiar with its use Participants were instructed to stop smoking from their quit date and continue with their allocated treatment for 12 weeks (ad libitum use of the e-cigarette), irrespective of any lapses to smoking

3) Nicotine patch and nicotine EC for 14 weeks. As above, but $18 \mathrm{mg} / \mathrm{mL}$ nicotine
Quit date, 1, 3,6 and 12 months

Continuous abstinence at 6 months with $\mathrm{CO}$ validation

Adverse events and biomarkers: Known side-effects associated with e-cigarette use and nicotine patch use; SAES

Other outcomes measured:

- Relapse

- Self-reported treatment adherence

- Tobacco withdrawal symptoms and urge to smoke

- Urge to vape

- Self-reported weight

- Concomitant medication

- Treatment cross-over

- Use of other smoking cessation support or medication

- Continued use of allocated treatment past 14 weeks

- Changes in shortness of breath, cough, asthma, COPD, and mental health problems

- Belief in ability to quit and remain tobacco-free

- Smoking identity and views on their allocated treatment for smoking cessation and whether they would recommend it to other people who smoke who want to quit

- In people still smoking at each follow-up call, outcomes were number of cigarettes smoked per day and reduction in smoking

- Participants allocated e-cigarettes were asked about their urge to vape; whether they changed devices or e-liquid, or both; whether they accessed any e-cigarette support

Study funding

Funding: Health Research Council of New Zealand. "The sponsor of the study had no role in the study design, data collection, data analysis, data interpretation, or writing of the report. The corresponding author had full access to all the data in the study and had final responsibility for the decision to submit for publication."

Author declarations

NW, CB, MV, GL, ML, and VP report grants from the Health Research Council of New Zealand, during the conduct of the study. NW, CB, MV, and VP report grants from Pfizer, outside of the submitted work. GL chairs the organization End Smoking New Zealand, which advocates for harm reduction approaches to tobacco control. E-cigarettes were purchased from a New Zealand e-cigarette online retailer (NZVAPOR, https://www.nzvapor.com/), e-liquid was purchased from Nicopharm, Australia (https:// www.nicopharm.com.au/), and nicotine patches were supplied by the New Zealand Government via 
Walker 2020 (Continued)

their contract with Novartis (Sydney, Australia). NZVAPOR also provided, at no cost to participants, online and phone support regarding use of the e-cigarettes. Neither NZVAPOR nor Nicopharm have links with the tobacco industry. None of the above parties had any role in the design, conduct, analysis, or interpretation of the trial findings, or writing of this publication.

Notes Study listed as ongoing study NCT02521662 in the 2016 review update

\section{Risk of bias}

\begin{tabular}{|c|c|c|}
\hline Bias & Authors' judgement & Support for judgement \\
\hline $\begin{array}{l}\text { Random sequence genera- } \\
\text { tion (selection bias) }\end{array}$ & Low risk & Computer-generated randomization sequence \\
\hline $\begin{array}{l}\text { Allocation concealment } \\
\text { (selection bias) }\end{array}$ & Low risk & $\begin{array}{l}\text { Quote: "We ensured allocation concealment because the statistician who gen- } \\
\text { erated the random allocation was not the person randomising participants." }\end{array}$ \\
\hline \multirow{2}{*}{$\begin{array}{l}\text { Blinding of participants } \\
\text { and personnel (perfor- } \\
\text { mance bias) } \\
\text { All outcomes }\end{array}$} & High risk & $\begin{array}{l}\text { Quote: "Participants and researchers collecting outcome data were masked to } \\
\text { the nicotine content of the e-liquid" but those allocated to patch only would } \\
\text { be aware they did not have an E-cigarette }\end{array}$ \\
\hline & & $\begin{array}{l}\text { Quote: "Third, while we attempted to minimise detection bias by masking the } \\
\text { nicotine content of the e-liquid, we were only } 30 \% \text { successful, and thus some } \\
\text { bias in favour of nicotine e-cigarettes could have occurred." }\end{array}$ \\
\hline $\begin{array}{l}\text { Blinding of outcome as- } \\
\text { sessment (detection bias) } \\
\text { All outcomes }\end{array}$ & Low risk & Biochemical validation \\
\hline $\begin{array}{l}\text { Incomplete outcome data } \\
\text { (attrition bias) } \\
\text { All outcomes }\end{array}$ & Low risk & $<50 \%$ lost to follow-up, similar rates of attrition between groups (within $20 \%$ ) \\
\hline $\begin{array}{l}\text { Selective reporting (re- } \\
\text { porting bias) }\end{array}$ & Unclear risk & $\begin{array}{l}\text { CO-verified abstinence at } 12 \text { months stated as a secondary outcome but data } \\
\text { are not reported in the main text. However, state in the appendix that too few } \\
\text { people in each group were followed up to } 12 \text { months (36/1124) so no data are } \\
\text { presented for this time point }\end{array}$ \\
\hline
\end{tabular}

Yingst 2020

\section{Study characteristics}

\begin{tabular}{ll}
\hline Methods & Design: Cross-over study \\
& Recruitment: Participants were recruited from people living with HIV/AIDS (PLWHA) (who smoked) \\
seeking care at the Penn State Health HIV Comprehensive Care Program \\
Setting: USA \\
Study start date:Not reported \\
Total N: $17 ; 41.2 \%$ female; mean age 49.1 (SD 8.8); mean cpd 16.9 (SD 7.9); mean CO 22.4 (13.1) \\
E-cigarettes use at baseline: not reported \\
Motivated to quit: No \\
Inclusion criteria:
\end{tabular}


Yingst 2020 (Continued)

- adult (age $\geq 18$ )

- smokers ( $\geq 10$ cigarettes daily)

- not planning to quit smoking

- documented history of a positive HIV status

Exclusion criteria: not reported

Interventions

\title{
EC: Cig-a-like; Refillable
}

Cig-a-like device (Blu), nicotine concentration $24 \mathrm{mg} / \mathrm{ml}$. Propylene glycol/ vegetable glycerin ratio $70 / 30$. Nicotine delivery $4.56 \mathrm{ng} / \mathrm{ml}$ after 20 puffs in 10 minutes

Button-operated device (eGO), nicotine concentration $36 \mathrm{mg} / \mathrm{ml}$. Propylene glycol/ vegetable glycerin ratio $70 / 30$. Nicotine delivery $6.9 \mathrm{ng} / \mathrm{ml}$ after 10 puffs in 5 minutes (refillable)

\author{
Outcomes \\ Visits: baseline, day 7 , day 14 , day 21 \\ CO measured (day 0, 7, 14, 21); adverse events (nausea, dizziness) \\ Also: Number of tobacco cigarettes smoked per day (self-report); EC puffs per day (self-report)
}

This study was supported by the National Institute on Drug Abuse of the National Institutes of Health
under Award Number P50DA036107 and the Center for Tobacco Products of the U.S. Food and Drug Ad-
ministration. JY is also funded by the Penn State Cancer Institute (PSCI) and TE is also supported by
U54DA036105. The content is solely the responsibility of the authors and does not necessarily repre-
sent the official views of the National Institutes of Health or the Food and Drug Administration

$\begin{array}{ll}\text { Author declarations } & \text { JF has done paid consulting for pharmaceutical companies involved in producing smoking cessation } \\ \text { medications, including GSK, Pfizer, Novartis, J\&J, and Cypress Bioscience. TE is a paid consultant in lit- } \\ \text { igation against the tobacco industry and the electronic cigarette industry and is named on a patent ap- } \\ \text { plication for a device that measures the puffing behavior of electronic cigarette users }\end{array}$

Notes New for 2021 update

\section{Risk of bias}

\begin{tabular}{lll}
\hline Bias & Authors' judgement & Support for judgement \\
\hline $\begin{array}{l}\text { Random sequence genera- } \\
\text { tion (selection bias) }\end{array}$ & Unclear risk & Used 2 ENDS in a random order - not enough information \\
\hline $\begin{array}{l}\text { Allocation concealment } \\
\text { (selection bias) }\end{array}$ & Unclear risk & Not reported \\
\hline
\end{tabular}

\begin{tabular}{l}
\hline Blinding of participants Low risk Unable to blind, but interventions judged equally intensive \\
and personnel (perfor- \\
mance bias) \\
All outcomes
\end{tabular}

\begin{tabular}{|c|c|c|}
\hline $\begin{array}{l}\text { Blinding of outcome as- } \\
\text { sessment (detection bias) } \\
\text { All outcomes }\end{array}$ & Low risk & Objective outcome- $\mathrm{CO}$ monitoring ( $\mathrm{CO}<10 \mathrm{ppm})$ \\
\hline $\begin{array}{l}\text { Incomplete outcome data } \\
\text { (attrition bias) } \\
\text { All outcomes }\end{array}$ & Low risk & No loss to follow-up \\
\hline $\begin{array}{l}\text { Selective reporting (re- } \\
\text { porting bias) }\end{array}$ & Unclear risk & Unclear what outcomes were prespecified \\
\hline
\end{tabular}


AE: adverse event; BMI: body mass index; CO: carbon monoxide; COT: cotinine; cpd: cigarettes per day; EC: electronic cigarette; ENDS: electronic nicotine delivery system; FTND: Fagerström Test for Nicotine Dependence; HRQoL: health-related quality of life; IQR: interquartile range; ITT: intention-to-treat; LTFU: lost to follow-up; MMT: methadone maintenance treatment; NEC: nicotine electronic cigarette; NRT: nicotine replacement therapy; PEC: placebo electronic cigarette; $P P(A)$ : point prevalence (abstinence); ppm: parts per million; SAE: serious adverse event; SD: standard deviation; SMI: serious mental illness; TQD: target quit date; UC: usual care

Characteristics of excluded studies [ordered by study ID]

\begin{tabular}{|c|c|}
\hline Study & Reason for exclusion \\
\hline Adkison 2013 & $\begin{array}{l}\text { Although this study uses a prospective cohort design, no data on EC use were collected at baseline, } \\
\text { with EC use data only being available at follow-up }\end{array}$ \\
\hline Al-Delaimy 2015 & $\begin{array}{l}\text { Observational study with no intervention provided - included in previous versions, but excluded } \\
\text { from } 2020\end{array}$ \\
\hline Anonymous 2019 & Commentary of included study (not primary study) \\
\hline Battista 2013 & Short-term EC use only \\
\hline Bianco 2019 & Ineligible intervention \\
\hline Biener 2015 & Cohort study, but EC use evaluated retrospectively only \\
\hline Biondi-Zoccai 2019 & Less than 1 week follow-up \\
\hline Biondi-Zoccai 2020 & Acute EC use only \\
\hline Borderud 2014 & $\begin{array}{l}\text { Observational study with no EC intervention provided - included in previous versions, but excluded } \\
\text { from } 2020\end{array}$ \\
\hline Brose 2015 & $\begin{array}{l}\text { Observational study with no EC intervention provided - included in previous versions, but excluded } \\
\text { from } 2020\end{array}$ \\
\hline Brown 2014a & Cross-sectional survey \\
\hline Bullen 2010 & Short-term EC use only \\
\hline Bullen 2018 & Withdrawn trial registry \\
\hline Caponnetto 2019 & Ineligible intervention \\
\hline Cavarretta 2019 & Less than 1 week follow-up \\
\hline Chaumont 2018 & Less than 1 week follow-up \\
\hline Chaumont 2019 & Ineligible intervention \\
\hline Chausse 2015 & Ineligible study design \\
\hline Choi 2014 & $\begin{array}{l}\text { Observational study with no EC intervention provided - included in previous versions, but excluded } \\
\text { from } 2020\end{array}$ \\
\hline Chorti 2012 & Short-term EC use only \\
\hline Collins 2019 & Ineligible intervention \\
\hline
\end{tabular}




\begin{tabular}{|c|c|}
\hline Study & Reason for exclusion \\
\hline Cook 2019 & Commentary of included study (not primary study) \\
\hline Cox 2019a & Short-term abstinence only ( $<6$ months) \\
\hline Czogala 2012 & Short-term EC use only \\
\hline D'Ruiz 2017 & Less than 1 week follow-up \\
\hline Dawkins 2012 & Short-term EC use only \\
\hline Dawkins 2013a & Short-term EC use only \\
\hline Dawkins 2014 & Short-term EC use only \\
\hline Douptcheva 2013 & $\begin{array}{l}\text { Longitudinal study, but no data are reported for smoking cessation or reduction or for adverse } \\
\text { events }\end{array}$ \\
\hline Dutra 2014 & Cross-sectional survey \\
\hline Eissenberg 2010 & Short-term EC use only \\
\hline Etter 2014 & $\begin{array}{l}\text { Observational study with no EC intervention provided - included in previous versions, but excluded } \\
\text { from } 2020\end{array}$ \\
\hline Farsalinos 2012 & Short-term EC use only \\
\hline Farsalinos 2013a & Included people that had already stopped smoking conventional cigarettes \\
\hline Farsalinos 2013b & Short-term EC use only \\
\hline Farsalinos $2013 c$ & Short-term EC use only \\
\hline Farsalinos 2013d & Short-term EC use only \\
\hline Flouris 2012 & Short-term EC use only \\
\hline Flouris 2013 & Short-term EC use only \\
\hline Gmel 2016 & Cohort study, but EC use only evaluated retrospectively \\
\hline Gottlieb 2019 & Commentary of included study (not primary study) \\
\hline Grana 2014b & $\begin{array}{l}\text { Observational study with no EC intervention provided - included in previous versions, but excluded } \\
\text { from } 2020\end{array}$ \\
\hline James 2016 & Follow-up at 12 weeks, $\mathrm{AE}$ data not collected \\
\hline Kasza 2013 & $\begin{array}{l}\text { Longitudinal study, but no data are reported for smoking cessation or reduction or for adverse } \\
\text { events }\end{array}$ \\
\hline Kouretas 2012 & Short-term EC use only \\
\hline Kousta 2019 & Commentary of included study (not primary study) \\
\hline Lechner 2015 & Less than 1 week follow-up \\
\hline
\end{tabular}




\begin{tabular}{|c|c|}
\hline Study & Reason for exclusion \\
\hline Lee 2014 & Cross-sectional survey \\
\hline Manzoli 2015 & $\begin{array}{l}\text { Observational study with no EC intervention provided - included in previous versions, but excluded } \\
\text { from } 2020\end{array}$ \\
\hline Marini 2014 & Short-term EC use only \\
\hline Mayor 2019 & Commentary of included study (not primary study) \\
\hline Meltzer 2017 & Ineligible intervention \\
\hline Miura 2015 & Tests a device which is not an EC \\
\hline NCT02487953 & Withdrawn trial registry \\
\hline NCT03036644 & Less than 1 week follow-up \\
\hline NCT03575468 & Ineligible intervention \\
\hline NCT04107779 & Less than 1 week follow-up \\
\hline Nolan 2016 & Short-term abstinence only (< 6 months) \\
\hline NTR6224 & Study terminated early, no usable results. Previously listed as ongoing \\
\hline Palamidas 2014 & Short-term EC use only \\
\hline Pearson 2012 & $\begin{array}{l}\text { Longitudinal study, but no data are reported for smoking cessation or reduction or for adverse } \\
\text { events }\end{array}$ \\
\hline Pokhrel 2013 & Cross-sectional survey \\
\hline Polosa 2014a & $\begin{array}{l}\text { Observational study with no EC intervention provided - included in previous versions, but excluded } \\
\text { from } 2020\end{array}$ \\
\hline Popova 2013 & Cross-sectional survey \\
\hline Prochaska 2014 & RCT but no EC intervention provided - included in previous versions, but excluded from 2020 \\
\hline Russo 2018 & Ineligible study design \\
\hline Schober 2014 & Short-term EC use only \\
\hline Siegel 2011 & $\begin{array}{l}\text { Retrospective survey of } 222 \mathrm{EC} \text { users that responded to a survey sent to } 5000 \text { new users of the 'Blu' } \\
\text { EC. Likely to be a self-selected sample }\end{array}$ \\
\hline Song 2020 & Ineligible patient population \\
\hline St.Helen 2020 & Wrong intervention \\
\hline Stein 2019 & Commentary of included study (not primary study) \\
\hline Stower 2019 & Ineligible study design \\
\hline Tsikrika 2014 & Short-term EC use only \\
\hline
\end{tabular}




\begin{tabular}{|c|c|}
\hline Study & Reason for exclusion \\
\hline Tucker 2018 & Short-term abstinence only (<6 months) \\
\hline Tzatzarakis 2013 & Short-term EC use only \\
\hline Vakali 2014 & Short-term EC use only \\
\hline Valentine 2016 & Less than 1 week follow-up \\
\hline Van Heel 2017 & Ineligible study design \\
\hline Vansickel 2010 & Short-term EC use only \\
\hline Vansickel 2012 & Short-term EC use only \\
\hline Vansickel 2013 & Short-term EC use only \\
\hline Vardavas 2012 & Short-term EC use only \\
\hline Vickerman 2013 & Cross-sectional survey \\
\hline Voos 2019 & Less than 1 week follow-up \\
\hline Voos 2020 & Ineligible study design \\
\hline Wagener 2014 & EC use for up to 1 week, but does not report on any adverse events \\
\hline Walele 2016a & RCT but follow-up too short \\
\hline Walele 2016b & RCT but follow-up too short \\
\hline Yan 2015 & Ineligible study design \\
\hline Yuki 2017 & Less than 1 week follow-up \\
\hline Zhang 2019 & Commentary of included study (not primary study) \\
\hline
\end{tabular}

EC: electronic cigarette

Characteristics of ongoing studies [ordered by study ID]

ACTRN12617001324303

\section{Study name}

Vaporised nicotine products versus oral forms of nicotine replacement therapy (NRT) products for tobacco smoking cessation among

low-socioeconomic status (low-SES) people who smoke

\begin{tabular}{ll}
\hline Methods & Parallel, single-blinded, \\
& Setting: Australia \\
& Recruitment: Not stated \\
\hline Participants & Target sample size: 868 \\
& Inclusion criteria:
\end{tabular}


- At least 18 years of age

- Current daily smoker

- Motivated and willing to make a quit attempt using medications (NRT/VNP)

- Speak English

- Able to provide verbal informed consent

- Receipt of government pension or allowance (proxy for low-SES)

- Have a phone we contact them on;

- Willing to complete 2 telephone check-in calls and baseline and follow-up telephone interviews

The term "current smoker" in this trial will refer to those who use either factory-made or roll-own cigarettes.

\section{Exclusion criteria:}

- Women who are pregnant, breastfeeding or planning to become pregnant in the next 12 months

- Current users of smoking cessation medications (i.e. NRT, bupropion [Zyban], clonidine, nortriptyline, electronic nicotine cigarettes )

- Those who are participating in another smoking cessation program or study

People will also be excluded if they report any of the following medical conditions in the previous 3 months: serious chronic lung diseases, arrhythmia, heart attack, stroke, or severe angina

Vaporised nicotine product (VNP) arm:
- Innokin Endura T18 Personal Vaporizer
- e-liquid nicotine ( $18 \mathrm{mg} / \mathrm{ml}$ nicotine) for 8 weeks
- 3 flavors will be offered: tobacco, strawberry, menthol
- Permitted to use the study product ad libitum throughout the day and encouraged to stop smok-
ing completely, or reduce smoking if unable to stop completely
- Participants will be provided with detailed instructions on how to use the e-cigarette device ef-
fectively
Oral nicotine replacement therapy (NRT) arm:
- 2 mg or 4 mg nicotine gum/lozenge for 8 weeks
- Quitline behavioral support
- Those receiving the lozenge will be instructed to use 9 - 15 lozenges per day, approximately 1 every
2 hours or when they have an urge to smoke
- Those receiving the gum will be instructed to use 10 to 20 pieces per day for the 2 mg gum and 4
to 10 pieces per day for the 4 mg gum, approximately 1 every 2 hours or when they have an urge
to smoke
- Participants will be provided with detailed instructions on how to use the NRT effectively and
encouraged to stop smoking completely, or reduce smoking if unable to stop completely
than 5 cigarettes) from the quit date ( 8 months from baseline)

Secondary outcomes measured at 2-week and 6-week check-in calls and 8-month follow-up

- Self-reported 7-day point prevalence abstinence

- Self-reported continuous abstinence: defined as self-report of smoking not more than 5 cigarettes from the designated quit date

- Self-reported number of cpd among people continuing to smoke

- Self-reported 30-day PPA at each follow-up (self-report of having smoked no cigarettes (not even a puff))

- Mean reduction in number of cigarettes smoked per day based on participant self-report 
- Proportion of participants that achieved a 50\% reduction of baseline cigarette consumption based on participant self-report (8 months only)

- Self-reported continued use of nicotine products to assess maintenance use and dual use (8 months only)

Weekly text message surveys and check-in calls 2 weeks and 6 weeks into the treatment period.

These check-in calls will also assess smoking status, short-term outcomes, and adverse events at these time points

\begin{tabular}{ll}
\hline Starting date & Anticipated start date: 30 April 2019 \\
\hline Contact information & Richard P Mattick, r.mattick@unsw.edu.au \\
& Alexandra Aiken, a.aiken@unsw.edu.au
\end{tabular}

Notes

Study name A pragmatic randomized partial cross-over clinical trial of nicotine vaporizers added to standard care for smoking cessation and relapse prevention (CARP) among priority populations with comorbidities

$\begin{array}{ll}\text { Methods } & \text { Randomized controlled trial } \\ \text { Setting: Australia } \\ \text { Recruitment: Not stated }\end{array}$

Participants

Target sample size: 810

Inclusion criteria:

- Diagnosed with or receiving treatment for a priority health conditions in the past 12 months

- Aged $18+$ years

- Currently smoke $10+$ cigarettes per day

- Has capacity to consent, able to understand participant materials and follow study instructions and comply with study procedures (e.g. sufficient English language ability, able to operate the vaporiser device)

- Willing to make a quit attempt at baseline according to randomized condition (Condition A to make quit attempt with nicotine vaporizer; Condition $B$ to make quit attempt without nicotine vaporizer)

- Has a referral to Quitline counseling and smoking cessation support program (standard care) but has not begun quit attempt (Note: Quitline referral can occur at time of study enrolment)

Exclusion criteria:

- Already begun quit attempt (i.e. post-quit day) at time of enrolment into trial or currently enrolled in another smoking cessation clinical trial or using varenicline or bupropion or used a nicotine vaporizer product in the last 30 days. NOTE: Use of nicotine replacement products not supplied in the trial (e.g. as part of quitline support) is not an exclusion criterion

- Currently pregnant or breast-feeding or an intention to be during trial participation period;

* A urinary pregnancy test will be required where pregnancy is suspected

* Participants will be advised appropriate contraception should be used to avoid pregnancy during the trial with ongoing contraception options discussed

- Has experienced cardiac-related chest pain, or another cardiovascular event or procedure in the last month, such as heart attack, stroke, insertion of stent, bypass surgery 
ACTRN12618000408280 (Continued)

- Hospitalized for a mental health condition in the last 30 days

- Currently being treated with oxygen therapy

- Diagnosed terminal illness (such as cancer) or debilitating condition that will limit ability to fully participate as determined by preregistration responses from participant or opinion of enrolling clinician

Interventions

Outcomes
- Arm 1) Referral to Quitline telephone smoking cessation counseling + Nicotine patches (15 $\mathrm{mg} / 16$-hr) delivered at baseline + refillable nicotine vaporizer device $(2 \times \mathrm{kits})+$ nicotine vaporising liquid (in high and low strength - high strength: nicotine 1.8\% in Vegetable Glycerine and purified water; low strength: nicotine $0.6 \%$ in Vegetable Glycerine and purified water). 1 patch to be applied daily to skin for up to 84 days. The vaporizer with nicotine liquid is to be used as needed up to $3.5 \mathrm{~mL}$ per day to treat withdrawal symptoms for up to 2 years (concurrently with patches for the first 84 days) to assist smoking cessation and relapse prevention. Participants start on highstrength nicotine liquid and may decrease their dose to low strength to assist with dose reduction prior to stopping use of the vaporizer.

- Arm 2) Referral to Quitline telephone smoking cessation counseling + Nicotine patches (15 $\mathrm{mg} / 16-\mathrm{hr}$ ) + participant's choice of either nicotine gum or nicotine lozenges (up to $800 \times 4 \mathrm{mg}$ pieces to be used up to 8 per day) delivered at baseline. Between 6 - 9 months post-baseline participants in Arm 2 who are smoking (either failed to quit or relapsed) will be offered: refillable nicotine vaporizer ( $2 \times$ kits) + nicotine vaporizing liquid (in high and low strength - high strength: nicotine $1.8 \%$ in Vegetable Glycerine and purified water; low strength: nicotine $0.6 \%$ in Vegetable Glycerine and purified water) to make a second quit attempt. Participants start on high-strength nicotine liquid and may decrease their dose to low strength to assist with dose reduction prior to stopping use of the vaporizer at the discretion of the participant. Participants will have until 2 years from baseline to use the vaporizer for smoking cessation and relapse prevention

Primary outcomes:

- Continuous abstinence from smoking from weeks 12 to 26 assessed at 26 weeks from baseline by self-report. Participants that self-report abstinence from smoking will be asked for a urine specimen for bioconfirmation. Urine specimens will be batch-tested for anabasine and cotinine at 6,12 and 21 month time points from baseline

Secondary outcomes:

- Continuous abstinence from smoking from weeks 12 to 52 , assessed at week 52 from baseline

- Continuous abstinence from smoking from weeks 12 to 104 , assessed by self-report at week 104 from baseline

- Continuous abstinence from smoking from weeks 40 to 52 , assessed by self-report at 52 weeks from baseline

- Continuous abstinence from smoking from weeks 92 to 104 , assessed by self-report at 104 weeks from baseline

- Number of adverse events measured by self-report at 12 weeks and 26 weeks from baseline

Abstinence is assessed through study-specific survey questions in Module CS Combustible Smoking Questions - administered through electronic survey or structured telephone interview. Participants that self-report abstinence from smoking will be asked for a urine specimen for bioconfirmation. Urine specimens will be batch-tested for anabasine and cotinine at 6,12 and 21 month time points

Starting date 5 June 2018

Contact information

Malcolm Brinn, m.brinn@uq.edu.au

Coral Gartner, c.gartner@uq.edu.au

Notes 


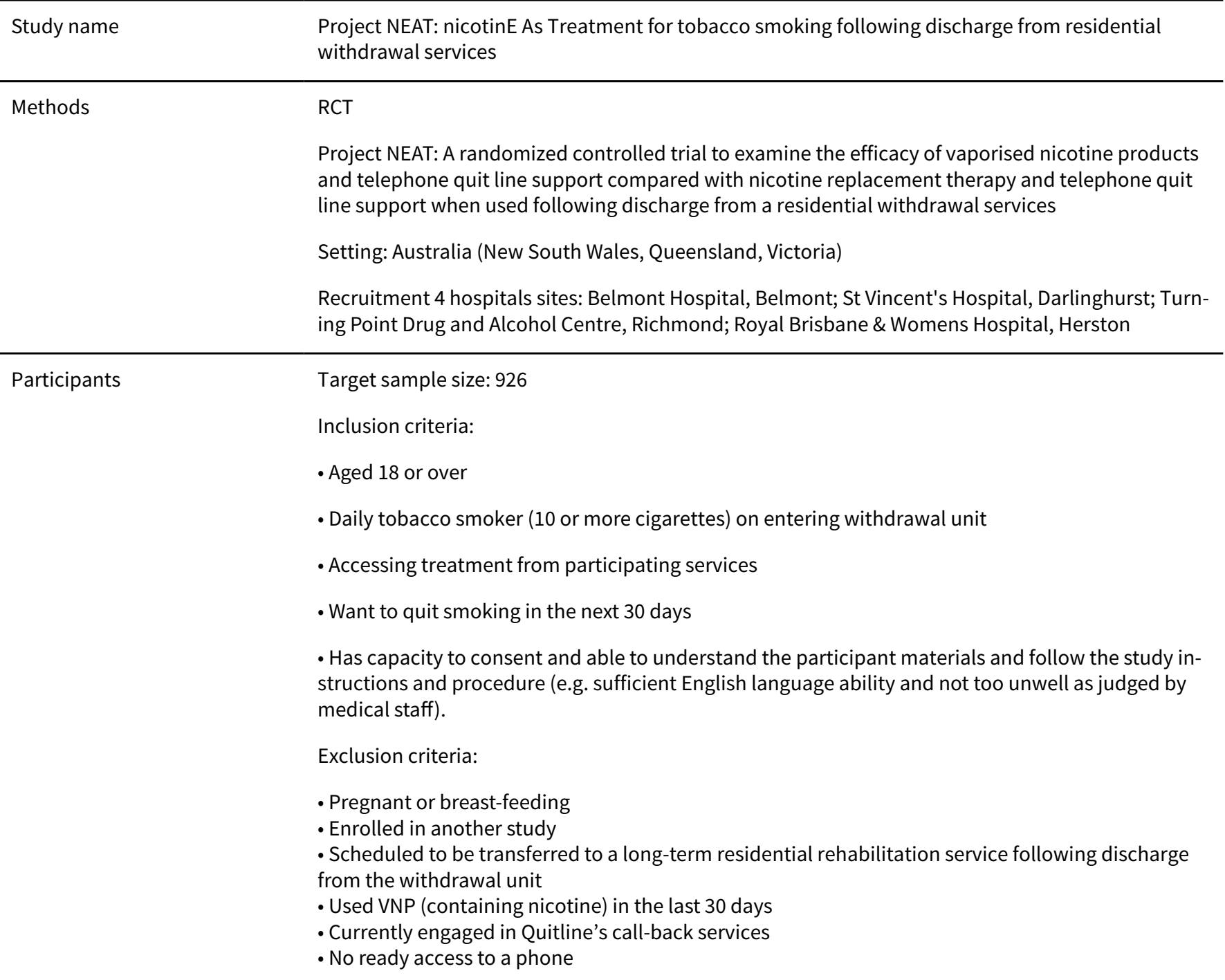

Condition Two: Current Best Practice Treatment for Tobacco Smoking (Combination Nicotine Replacement Therapy and Quitline)

\section{Outcomes}

9 months after inpatient withdrawal unit discharge:

Self-reported 7 months continuous abstinence from tobacco smoking

Biochemically-verified 7-month continuous abstinence from tobacco smoking

3 and 9 months after inpatient withdrawal unit discharge:

30-day point prevalence abstinence

7-day point prevalence abstinence

Abstinence from all nicotine/ tobacco products

Prof Billie Bonevski, Billie.Bonevski@newcastle.edu.au 
ACTRN12619001787178 (Continued)

Notes

Funding: National Health and Medical Research Council (grant number: G1800272), Canberra ACT 2601.

\section{Begh 2019}

\section{Study name}

Examining the effectiveness of general practitioner and nurse promotion of electronic cigarettes versus standard care for smoking reduction and

abstinence in hardcore smokers with smoking-related chronic disease: protocol for a randomized controlled trial

\section{Methods}

Individually randomized, blinded, 2-arm trial

Setting: General practices, England

Recruitment: Primary care registries
Target sample: 320 (160 per arm)

Inclusion criteria:

- Participant is willing and able to give informed consent for participation in the study

- Aged 18 years or above

- Current smoker with a value of at least 10 ppm for exhaled $\mathrm{CO}$ and smokes a minimum of 8 cigarettes/8 $\mathrm{g}$ of tobacco per day (including pipe, cigars or tobacco roll-ups)

- Diagnosed with 1 or more of the following chronic conditions: ischaemic heart disease, peripheral vascular disease, hypertension, diabetes mellitus (type 1 and type 2), stroke, asthma, COPD, chronic kidney disease, depression, schizophrenia, bipolar disorder or other psychoses

Exclusion criteria:

- GP believes that switching to e-cigarettes would not benefit the patient given their current medical condition

- Currently using e-cigarettes, nicotine replacement therapy or other cessation therapies (e.g. bupropion, nortriptyline or varenicline)

- Plans to stop smoking before or at the annual review

- Currently enrolled in another smoking-related study or other study where the aims of the studies are incompatible

- Cannot consent due to mental incapacity

- Pregnant, breastfeeding or planning to become pregnant during the course of the study

- Control: No additional support beyond standard care

- Intervention: will receive GP- or nurse-led brief advice about e-cigarettes, an e-cigarette starter pack with accompanying practical support booklet, and telephone support from experienced vapers and online video tutorials
Months 2, 8

Primary outcomes:

- 7-day PPA from smoked tobacco at 2 months; Self-reported abstinence from smoking-not even a puff-in the past 7 days, accompanied by a salivary anabasine concentration of $<1 \mathrm{ng} / \mathrm{ml}$; exhaled $\mathrm{CO}$ as verification of abstinence $(\mathrm{CO}<10 \mathrm{ppm})$ used, as necessary.

Secondary outcomes:

- Smoking reduction

- 7-day PPA and prolonged abstinence at 8 months; 
Begh 2019 (Continued)

- Participant recruitment and follow-up,

- Participant uptake and use of e-cigarettes,

- Nicotine intake,

- Contamination of randomization and practitioner adherence to the delivery of the intervention

\begin{tabular}{ll}
\hline Starting date & November 2016 \\
\hline Contact information & Rachna Begh, rachna.begh@phc.ox.ac.uk \\
\hline Notes & \\
\hline
\end{tabular}

\section{Berlin 2019}

Study name

Randomized, placebo-controlled, double-blind, double-dummy, multicentre trial comparing electronic cigarettes with nicotine to varenicline and to electronic cigarettes without nicotine: the ECSMOKE trial protocol

Methods

3-arm randomized, placebo-controlled, multicentre, double-blind, double-dummy, parallel groups, phase III type trial

Setting: Smoking cessation clinics of both academic and community hospitals

Recruitment is either local (a) directly by the centres or centralized (b) using a web page and a centralized study-specific phone number and email address

- People who smoke intending to quit smoking are recruited by advertisement in pharmacies, physicians' offices situated in the catchment area of each investigator's centre, by local newspapers and in public places of the centres' healthcare facilities

- Candidates to participate can register by the study's website, unique email address and phone number. Registration is followed by a phone screening before dispatching to the study centres. Only 1 person by household will be recruited

Participants

Estimated enrolment: 650 participants

Inclusion criteria:

- People who smoke, at least $10 \mathrm{cpd}$ (factory-made or roll-your-own) in the past year

- Aged 18 - 70 years

- Motivated to quit, defined as a score $>5$ on a visual rating scale ranging from 0 (not motivated at all) to 10 (extremely motivated)

- Signed written informed consent

- Understanding and speaking French

- Women of childbearing age can be included if they use an effective contraceptive method: either hormonal contraception or an intrauterine device started at least 1 month before the first research visit

- Individual affiliated to a health insurance system

- Previous failure of NRT for smoking cessation

Exclusion criteria:

- Any unstable disease condition within the last 3 months defined by the investigator as major change in symptoms or treatments, such as recent myocardial infarction, unstable or worsening angina, severe cardiac arrhythmia, unstable or uncontrolled arterial hypertension, recent stroke, cerebrovascular disease, obliterative peripheral arterial disease, cardiac insufficiency, diabetes, hyperthyroidism, pheochromocytoma, severe hepatic insufficiency, history of seizures, severe depression, COPD

- Any life-threatening condition with life expectancy of $<3$ months 
Berlin 2019 (Continued)

- Alcohol use disorder defined as a score $\geq 10$ on the Alcohol Use Disorders Identification Test (AUDIT)-C questionnaire (see below)

- Abuse of or dependence on illegal drugs in the last 6 months, revealed by medical history

- Regular use of tobacco products other than cigarettes

- Current or previous (last 6 months) use of EC

- Pregnant women

- Breastfeeding women

- Protected adults

- Current or past 3 months participation in another interventional research

- Current or past 3 months use of smoking cessation medication such as varenicline, bupropion, NRTs

- Known lactose intolerance (placebo tablets contain lactose)

- Hypersensitivity to the active substance or to any of the excipients

- Known severe renal failure

Interventions

A) EC without nicotine (ECwON) plus placebo tablets of varenicline $(0.50 \mathrm{mg})$ administered by oral route: placebo condition;

B) EC with nicotine (ECWN) plus placebo tablets of varenicline: $E C W N$ condition. $V$

C) Reference: ECwoN plus 0.5 mg varenicline tablets: varenicline condition. Varenicline administered according to the marketing authorization

E-cigarette details:

- EC device Mini iStick kit (20 W) Eleaf, clearomiser: GS Air M with resistance of $1.5 \mathrm{ohm}$. To keep the blinding, the clearomizer's Pyrex window is of grey Colour not allowing to distinguish the coloration of the e-liquid containing nicotine. Liquid for EC is manufactured by GAIATREND SARL (www.gaiatrend.fr/fr/)

- All participants will be delivered a short manual and a video specifically developed for this study explaining the use of EC. At each visit, participants receive verbal counseling about the use of the EC device and answers to their questions about handling the EC device

Behavioural support:

- Brief behavioral smoking cessation counseling for all participants is administered at all visits by the investigators specialized in smoking cessation. It is based on the national guidelines for smoking cessation

Treatment duration: 1 week +3 months

Outcomes

Week 2, 4, 8, 10, 12, 24 after target quit day

Primary outcome:

- Continuous smoking abstinence rate (CAR) (abstinence from conventional/combustible cigarettes) during the last 4 weeks (weeks $9-12$ ) of the treatment period of 3 months

Secondary outcomes:

- Safety profile

- PPA rate

- CAR confirmed by urinary anabasine concentration

- Changes in cpd consumption

- Craving for tobacco and withdrawal symptoms with respect to baseline 
Berlin 2019 (Continued)

Notes

Caponnetto 2014

Study name Smoking cessation and reduction In schizophrenia (the SCARIS study)

Methods

3-arm prospective 12-m randomized controlled trial investigating efficacy and safety of EC

Setting: psychiatric and smoking cessation centres, Italy

Recruitment: local newspapers and radio/television advertisements

\begin{tabular}{|c|c|}
\hline Participants & $\begin{array}{l}153 \text { participants } \\
\text { Inclusion criteria } \\
\text { - Schizophrenic in stable phase of illness } \\
\text { - Smoked at least } 10 \mathrm{cpd} \text { over previous } 5 \text { years } \\
\text { - Aged } 18 \text { - } 65 \\
\text { - In good general health } \\
\text { - Not currently attempting to quit smoke or wishing to do so in next } 6 \mathrm{~m} \\
\text { Exclusion criteria } \\
\text { - Use smokeless tobacco or NRT } \\
\text { - Pregnant or breastfeeding } \\
\text { - Current or recent ( } 1 \text { yr) history of drug or alcohol abuse } \\
\text { - Other significant co-morbidities }\end{array}$ \\
\hline Interventions & $\begin{array}{l}\text { 12-wk supply of: } \\
\text { - EC, high nicotine ( } 24 \mathrm{mg}) \\
\text { - EC, no nicotine ( } 0 \mathrm{mg} \text {, with tobacco aroma) } \\
\text { - PAIPO nicotine-free inhalator }\end{array}$ \\
\hline Outcomes & $\begin{array}{l}\text { Follow-up visits at } 4,8,12,24 \text { and } 52 \text { weeks } \\
\text { Outcome measures: } \\
\text { - Smoking cessation } \\
\text { - Smoking reduction ( } \geq 50 \% \text { from baseline) } \\
\text { - Adverse events } \\
\text { - Quality of life } \\
\text { - Neurocognitive functioning } \\
\text { - Participant perceptions and satisfactions with products }\end{array}$ \\
\hline Starting date & September 2014 \\
\hline Contact information & Pasquale Caponnetto, p.caponnetto@unict.it \\
\hline
\end{tabular}




\section{Caponnetto 2020}

\section{Study name}

Non-inferiority trial comparing cigarette consumption, adoption rates, acceptability, tolerability, and tobacco harm reduction potential in smokers switching to Heated Tobacco Products or electronic cigarettes: Study protocol for a randomized controlled trial

\begin{tabular}{ll}
\hline Methods & RCT \\
12 weeks
\end{tabular}

\begin{tabular}{ll}
\hline Participants & 220 healthy people who smoke tobacco cigarettes \\
\hline Interventions & Arm 1 - Heated Tobacco Products (HTPs) \\
& Arm 2 - E-cigarettes (ECs) \\
\hline Outcomes & $\begin{array}{l}\text { 12-week study. Follow-up 24 weeks } \\
\text { Biochemically-verified self-reported continuous abstinence at } 12 \text { weeks from the previous visit }\end{array}$ \\
& $\begin{array}{l}\text { Secondary outcomes will include: smoking reduction from baseline, adoption rates and product } \\
\text { acceptability, tolerability, changes in step test values and in the level of selected biomarkers of ex- } \\
\text { posure in exhaled breath (i.e. eCO) and in spot urine samples }\end{array}$ \\
& $\begin{array}{l}\text { A follow-up visit at 24 weeks to review product usage and smoking behavior under naturalistic con- } \\
\text { dition of use }\end{array}$ \\
\hline Starting date & Recruitment May 2019, enrolment is expected to be completed in November 2019 \\
& Results to be reported in 2020 \\
\hline Contact information & Pasquale Caponnetto, p.caponnetto@unict.it \\
\hline Notes & NCT03569748 \\
\hline
\end{tabular}

\section{Fraser 2015}

$\begin{array}{ll}\text { Study name } & \text { An open-label randomized pragmatic policy trial examining effectiveness of short-term use of nico- } \\ \text { tine replacement therapy (NRT) vs short- or long-term use of NRT vs short- or long-term use of NRT } \\ \text { or electronic nicotine delivery systems for smoking cessation in cigarette smokers }\end{array}$

$\begin{array}{ll}\text { Methods } & \text { Phase } 3 \text { blinded RCT } \\ & \text { Setting: Australia } \\ & \text { Recruitment: commercial market research panel }\end{array}$

\section{Participants}

Target sample size: 1600

- Current daily smoking (at least $6 \mathrm{cpd}$ )

- Can read and understand English

- Agree to try samples of nicotine products

- Willing to complete surveys

- 18 years or older

Exclusion criteria:

- If currently treated for serious medical condition, 
Fraser 2015 (Continued)

- Pregnant or planning to become pregnant or breast-feed in next $12 \mathrm{~m}$
- a) Factsheet explaining relative harm of NRT compared to smoking, free sample of NRT, participant chooses preferences, has free for 3 weeks then offered at subsidized rate for further $6 \mathrm{~m}$

- b) As (a), but with additional information provided

- c) As (a), but additional information on electronic cigarettes and emphasis on cessation, and may select electronic cigarettes as well as NRT

\begin{tabular}{ll}
\hline Outcomes & m and 12 m, self-report \\
& - Continuous abstinence \\
- NRT and EC use & - Interest in quitting smoking and in quitting NRT \\
- Cigarette consumption \\
- Product orders and use \\
- Quit attempts \\
\hline Starting date & February 2014 \\
\hline Contact information & Coral Gartner, c.gartner@uq.edu.au \\
\hline Notes & \\
\hline
\end{tabular}

\section{ISRCTN13288677}

\section{Study name}

Can electronic cigarettes and nicotine replacement treatment help reduce smoking in smokers who struggle to quit?

\begin{tabular}{ll}
\hline Methods & Pilot single-centre randomized control trial \\
Setting: Queen Mary University of London, UK \\
Recruitment method not specified. \\
\hline Target sample size: 200 \\
Inclusion criteria: \\
- 18 years or older \\
- Able to provide written informed consent \\
- History of failed quit attempts using stop-smoking medications or stop smoking services, or both \\
- Willing to use their allocated harm-reduction strategy for at least 4 weeks \\
Exclusion criteria: \\
- Women who are pregnant or breastfeeding \\
- Unable to read/write/understand English \\
- Currently using EC or any stop-smoking products \\
- Taking part in other interventional research \\
- Have a strong preference to use or not to use NRT or EC
\end{tabular}

\section{1) NRT arm:}

- Will be shown and explained the NRT products available and encouraged to choose a product or product combination that suits their needs 
ISRCTN13288677 (Continued)

- Will receive a letter of recommendation as per standard practice and collect their chosen products at local pharmacies

- Product use will be supervised and adjusted (if required) as part of the behavioral support package. As per local standard practice, NRT will be provided for up to 8 weeks

\section{2) EC arm:}

- Will be shown and explained different EC products commonly used and asked to obtain the product of their choice, either using a voucher for up to GBP 35 to purchase EC at a local vape shop, purchase from other suppliers and claim a refund of up to GBP 35 upon providing a valid receipt, or choose from a limited selection at the smoking cessation clinic

- Will be encouraged to try different products and liquids if the first purchase does not meet their needs, but after the initial purchase, participants will fund further supplies themselves (this is to mimic the provision of starter packs, an approach that is most likely to be used by routine services)

Outcomes

Participants contacted by phone at 1 week, 4 weeks and 24 weeks after the initial screening session

Primary outcomes:

- Cigarette consumption per day, assessed by self-report in the follow-up survey created for the purpose of the study at 1, 4 and 24 weeks post-quit date/preparation date. Those who report $\geq$ $50 \%$ smoking reduction will be validated with a $\mathrm{CO}$ reading in the clinic

Secondary outcomes:

- Use of allocated harm-reduction strategies

- Strategy ratings

- Changes in smoking behavior

- Proportion of people still using allocated strategy at 6 months

Starting date January 2017

Contact information Marzena Orzol, m.orzol@qmul.ac.uk

Katie Myers-Smith, katie.smith@qmul.ac.uk

Notes

\section{ISRCTN61193406}

Study name Do e-cigarettes help smokers quit when not accompanied by intensive behavioral support? A multi-center randomized controlled trial

Methods $\quad$ RCT

Setting: UK

Multicenter. Participants will be recruited mainly from hospitals and GP practices across the UK by the Clinical Research Network. The study is being organized by Queen Mary University of London (QMUL)

Researchers from QMUL will provide the study treatment and conduct follow-up calls

1170 people who smoke tobacco cigarettes
Inclusion criteria:
- Adult daily smokers who are motivated to stop smoking


ISRCTN61193406 (Continued)

- Must own a mobile phone and be willing to try either an online or texting treatment package, or both, or an e-cigarette with or without telephone support.

- Be happy to receive follow-up calls

- Be able to read/write/understand English

Exclusion criteria:

- Women who are pregnant

- Currently using an e-cigarette
1. Control: NHS Quit Now program (QN)

2. E-cigarette starter pack with no ongoing support (EC)

3. EC starter pack with helpline support (EC+)

The study will aim to use a refillable EC that is similar to the type used in a previous EC trial (One Kit - Innokin, UK Ecig Store), and one that is compliant with UK regulations, and not produced by a tobacco company

Outcomes

Primary outcome measure:

Sustained smoking cessation at 6 months post-TQD. This is measured by asking participants if they have smoked since their TQD at the 6-month follow-up. To be counted as a 'quitter', participants must report smoking no more than 5 cigarettes since 2 weeks post-TQD with no smoking in the previous week, validated by carbon monoxide $(\mathrm{CO})$ reading of $<8 \mathrm{ppm}$. Participants lost to follow-up will be counted as smokers

Secondary outcome measures:

- Validated sustained abstinence rates measured by asking smoking status and taking a carbon-monoxide reading at 12 months post-TQD

- Validated sustained abstinence rates between 6 and 12 months, measured by asking smoking status and taking a carbon-monoxide reading at 6 and 12 months

- Self-reported 7-day point-prevalence abstinence, measured by asking smoking status in last 7 days at 4 weeks, 6 months and 12 months post-TQD

- Cigarette consumption in non-abstainers by vaping status, measured by questionnaire at four weeks, 6 and 12 months

- Frequency and severity of urges to smoke and withdrawal symptoms, measured by questionnaire at 4 weeks post-TQD.

- Weight, measured by asking weight at 4 weeks, 6 months and 12 months post-TQD

- Respiratory symptoms, measured by questionnaire, at 4 weeks, 6 months and 12 months postTQD

- Treatment adherence and ratings, measured by questionnaire at 4 weeks (and 6 and 12 months for EC arms)

- Adverse reactions to EC, measured by questionnaire at 4 weeks, 6 and 12 months post-TQD

- Cost-effectiveness of the interventions, measured by questionnaires at baseline, 6 and 12 months

- Smokers' and health-care professionals views and opinions of the helpline, measured by one-off qualitative interviews separate to the main trial.

Starting date

Overall trial start date: 01 September 2020

Trial end date: 31 May 2024

Not yet recruiting. Last edited 12 August 2020

Dr Katie Myers Smith, katie.smith@qmul.ac.uk 
ISRCTN61193406 (Continued)

Notes

Klonizakis 2017

Study name Smokers making a quit attempt using e-cigarettes with or without nicotine or prescription nicotine replacement therapy: impact on cardiovascular function (ISME-NRT) - a study protocol

Methods Pragmatic, 3-group, randomized, assessor-blinded, single-centre trial

Setting: Centre for Sport and Exercise Science (CSES) of Sheffield Hallam University, UK

Recruitment: From the community in the wider Sheffield area will be by: i) low-cost newspaper and post-office advertisement, ii) posters in local pharmacies, libraries, mosques, churches, and clubs, iii) social media or search engine advertisement (Facebook, Google ads) iv) notices in newsletters or participation in outreach events of community organizations (such as Sheffield U3A and AGE UK), iv) a study website, and v) out-reach events in local ethnic community centres or places of worship

Estimated enrolment: 258 participants (86 participants arm)
Inclusion Criteria:
- Age > 18 years of either sex
- People who smoke (at least 10 cpd for the past year)
- Willing (by declaration) to attempt quit smoking by using the NHS services or e-cigarettes
Exclusion Criteria:
- Inability to walk
- Recent (within 6 months) cardiovascular disease event (e.g. stroke, myocardial infarction) or car-
- diac surgery
- Insulin-controlled diabetes mellitus or with co-existing skin conditions, leg ulcers, vasculitis or
- Pregnancy
- Requiring major surgery during the course of the study)
- Current daily use of e-cigarettes
- Currently undertaking a cessation attempt supported by a smoking cessation clinic
- Unable to give informed consent

- a) Complimentary e-cigarette equipment and refills (Tornado V5, Joyetech, Shenzhen, China) at allocation stage, together with instructions on the correct usage of e-cigarettes. They will also receive behavioral support for a 3-month period. The nicotine strength of Group A cartridges will be up to $18 \mathrm{mg} / \mathrm{ml}$ nicotine strength

- b) As a), but with nicotine-free liquid

- c) Referral to NHS smoking cessation clinics and will receive NRT in conjunction with behavioral support

\section{Outcomes}

Follow-up: Within 3 days of "quit date", 3 and 6 months past quit date

Outcome measures:

- Macro-vascular function (FMD assessment)

- Micro-vascular function

- Smoking status at 3 and 6 months, self-reported and biochemically validated by exhaled air measurement of $<10 \mathrm{ppm} \mathrm{CO}$ 
Klonizakis 2017 (Continued)

- Change in CVD risk using Q-risk assessment

- Health Economic effects using EQ5D-L

- Total cholesterol and High Density lipoprotein via fingerprick blood sample

- Participant experiences' assessment

\begin{tabular}{ll}
\hline Starting date & 24 April 2017 \\
\hline Contact information & Markos Klonizakis, m.klonizakis@shu.ac.uk
\end{tabular}

Notes

\section{Murray 2020}

Study name

Yorkshire Enhanced Stop Smoking (YESS) study: a protocol for a randomized controlled trial to evaluate the effect of adding a personalized smoking cessation intervention to a lung cancer screening program

Methods RCT

Setting: Yorkshire, UK

\section{Participants}

Anticipated recruitment: 1040 people who smoke tobacco cigarettes

Participants are aged $55-80$, registered with a general practitioner (GP) in the Leeds Clinical Commissioning Group area and registered as a current or ex-smoker in primary care databases

Inclusion criteria:

- Attended an lung health check (LHC) and consent to participate in the Yorkshire Lung Screening Trial (YLST)

- have smoked within the last month

- have an exhaled carbon monoxide (CO) reading $\geq 6 \mathrm{ppm}$

- have agreed to see an SCP on the mobile unit

Exclusion criteria:

- Any individual who does not have an LDCT scan

- or is unable to provide informed consent SC support over 4 weeks comprising behavioral support, pharmacotherapy and/or a commercially-available e-cigarette

Arm 2: continued standard best practice.

\section{Outcomes}

Follow-up contact will be requested at 4 weeks, 3 months and 12 months, with a 2-week window to accommodate participant availability

The primary objective is to measure 7-day point prevalent carbon monoxide (CO)-validated SC after 3 months

Secondary outcomes include CO-validated cessation at 4 weeks and 12 months, self-reported continuous cessation at 4 weeks, 3 months and 12 months, attempts to quit smoking and changes in psychological variables, including perceived risk of lung cancer, motivation to quit smoking tobacco, confidence and efficacy beliefs (self and response) at all follow-up points 
Murray 2020 (Continued)

Contact information

Professor Rachael L Murray; rachael.murray@nottingham. ac.uk

\section{Notes}

\section{NCT01842828}

\begin{tabular}{|c|c|}
\hline Study name & Spain-UK-Czech E-cigarette Study (SUKCES) \\
\hline \multirow[t]{3}{*}{ Methods } & Randomized controlled trial, open-label pilot study \\
\hline & Setting: smoking cessation clinics in London, Madrid and Prague \\
\hline & Recuitment: via smoking cessation clinics \\
\hline \multirow[t]{8}{*}{ Participants } & 220 people who smoke, seeking help to quit \\
\hline & Inclusion criteria: \\
\hline & - 18 or older \\
\hline & - Want help to quit \\
\hline & Exclusion criteria: \\
\hline & - Pregnant or breastfeeding; \\
\hline & - Enrolled in other research; \\
\hline & - Currently using EC \\
\hline \multirow[t]{2}{*}{ Interventions } & - Standard care plus 4 weeks EC supply \\
\hline & - Standard care only \\
\hline \multirow[t]{5}{*}{ Outcomes } & - CO-validated continuous abstinence at 4 and 24 weeks post-TQD \\
\hline & - Withdrawal symptoms at 1 and 4 weeks post-TQD \\
\hline & - EC use \\
\hline & - EC taste and satisfaction compared to conventional cigarettes \\
\hline & - Adverse events \\
\hline Starting date & December 2013 \\
\hline Contact information & Peter Hajek, p.hajek@qmul.ac.uk \\
\hline
\end{tabular}

NCT01989923

\begin{tabular}{ll}
\hline Study name & Smoking cessation in women with gynecological conditions \\
\hline Methods & Randomized controlled trial, open-label feasibility study \\
& Setting: hospital clinic, USA \\
& Recruitment: in clinic \\
\hline
\end{tabular}

Participants

30 women who smoke with cervical dysplasia 
NCT01989923 (Continued)

Inclusion criteria:

- Women who smoke at least $10 \mathrm{cpd}$ over past year

- Diagnosis of cervical dysplasia, cervical cancer, and lower genital tract dysplasia and cancer

- Aged $18-65$

Exclusion criteria:

- Previous diagnoses or treatment for cancer (except for non-melanoma skin cancer)

- Stroke, heart disease, heart attack, or irregular heart beat

- Pregnancy and lactation

- Plan to continue to use other nicotine as well as study products

- Uncontrolled hypertension

- Using other stop-smoking medication

- Taking prescription medicine for depression or asthma

Interventions

- NRT patch ( $21 \mathrm{mg}$ for first 3 weeks, $14 \mathrm{mg}$ for 2 nd 3 weeks) plus nicotine gum ( $2 \mathrm{mg}$ ) or lozenges (2 mg) for 6 weeks

- EC device ('Blu' Cig) with refills to last 6 weeks, number provided based on packs smoked a day $x$ 1.5. Strength of EC reduced at 3 weeks

Both groups receive identical cessation counseling

\begin{tabular}{ll}
\hline Outcomes & At 6 and 12 weeks via survey: \\
& Cpd \\
& PPA at 7 and 30 days \\
& - Smoking cessation \\
& Participants' attitudes and beliefs towards treatments \\
\hline Starting date & June 2013 \\
\hline Contact information & Laura A Beebe, laura-beebe@ouhsc.edu \\
\hline Notes & \\
\hline
\end{tabular}

NCT02004171

\begin{tabular}{ll}
\hline Study name $\quad$ Electronic cigarettes or nicotine inhaler for smoking cessation \\
\hline
\end{tabular}

Methods

Randomized controlled trial, open-label safety/efficacy study

Setting and recruitment not specified, USA

Participants 40 participants
Inclusion criteria:
- $18-60$ years old
- Meet DSM-IV criteria for nicotine dependence
- Seeking treatment for smoking cessation
- smoking at least $15 \mathrm{cpd}$
Exclusion criteria:


NCT02004171 (Continued)

- DSM-IV diagnosis of schizophrenia, schizoaffective disorder, or bipolar disorder

- Current diagnosis of major depressive disorder

- Current diagnosis for other psychiatric disorders that may require intervention over course of study

- Receiving treatment for nicotine dependence

- Pregnancy, lactation, or chance of pregnancy

- Unstable medical condition

- Substance abuse diagnosis

- Use of cannabis or alcohol on more than 20 days in past 30 days

- Suicide risk
4 weeks:

- ECs (2nd generation) with $24 \mathrm{mg}$ nicotine cartridges, 1 - 2 cartridges daily

- Nicotine inhaler with $10 \mathrm{mg}$ cartridges, max 16 cartridges per day

\begin{tabular}{ll}
\hline Outcomes & Over 4 weeks: \\
& - cpd \\
& Withdrawal \\
& Benefits from smoking cessation (breathing, sense of taste and smell, physical fitness) \\
& - Bdverse events \\
\hline Starting date & December 2013 \\
\hline Contact information & Barney Vaughan, vaughan@nyspi.columbia.edu \\
\hline Notes & \\
\hline
\end{tabular}

\section{NCT02124187}

\begin{tabular}{ll}
\hline Study name & Smoking cessation and reduction in depression (SCARID) \\
\hline Methods & 3-arm prospective 12-m randomized controlled trial investigating efficacy and safety of ECs \\
\hline
\end{tabular}

Participants

129 participants

Inclusion criteria:

- Diagnosis of major depressive disorder (MDD) (according to DSM-5 criteria)

- Smoke $\geq 10 \mathrm{cpd}$ (for at least the past 5 years)

- age 18 - 65 years

- In good general health

- Unwilling to quit smoking in the next 30 days

Exclusion criteria:

- Use of smokeless tobacco or NRT or other smoking cessation therapies

- Pregnancy or breastfeeding

- Current or recent ( $<1 \mathrm{yr}$ ) past history of alcohol or drug abuse or both

- Active suicidal intention 
NCT02124187 (Continued)

- Other significant co-morbidities according to the Investigator's clinical assessment (e.g. cancer, acute myocardial infarction, unstable angina, severe cardiac arrhythmia, recent cerebrovascular incident, or severe atherosclerosis)

\begin{tabular}{ll}
\hline Interventions & 12-wk supply of: \\
& - EC $24 \mathbf{~ m g ~ n i c o t i n e ~}$ \\
& EC 0 mg nicotine \\
& Nicotine-free inhalator
\end{tabular}

\begin{tabular}{ll}
\hline Outcomes & Follow-up visits at 4, 8, 12, 24 and 52 weeks \\
& Outcome measures: \\
& - Smoking cessation \\
& - Smoking reduction ( $\geq 50 \%$ from baseline) \\
- Adverse events & Quality of life \\
- Neurocognitive functioning \\
- Participant perceptions and satisfaction with products
\end{tabular}

\section{Starting date}

Contact information

\section{February 2015}

Pasquale Caponnetto p.caponnetto@unict.it

\section{Notes}

\section{NCT02398487}

Study name

Head-to-head comparison of personal vaporizers versus cig-a-like: prospective 6-month randomized control design study (VAPECIG 2)

\section{Methods}

\section{Randomized parallel-assignment open-label trial}

Setting: Italy, community

\section{Participants}

Estimated enrolment: 200

Inclusion criteria:

- (People who smoke) in good general health

- Committed to follow trial procedures

Exclude if:

- Recent vaping history (stopped vaping $<3$ months ago)

- Use of any other form of non-combustible nicotine-containing products (chewable tobacco or nicotine replacement therapy)

- Symptomatic cardiovascular disease

- Clinical history of asthma and COPD

- Regular psychotropic medication use

- Current or past history of alcohol abuse

- Use of smokeless tobacco or nicotine replacement therapy

- Pregnancy or breastfeeding. 
NCT02398487 (Continued)

Interventions Comparison between 2 types of EC; 'personal vaporizers' and 'cig-a-like'

\begin{tabular}{ll}
\hline Outcomes & 24 weeks: \\
& - Smoking cessation \\
& Smoking reduction \\
\hline Starting date & October 2014 \\
\hline Contact information & Riccardo Polosa \\
\hline
\end{tabular}

NCT02527980

Study name E-cigarettes: dynamic patterns of use and health effects

$\begin{array}{ll}\text { Methods } & \text { Prospective observational study } \\ \text { Setting: community, USA } \\ \text { Recruitment: People who smoke and dual EC and cigarette users }\end{array}$

Estimated enrolment: 450
Inclusion criteria:
- $\geq 18$ years old
- No plans to quit smoking and/or EC use in the next 30 days
- Not currently taking smoking cessation medication
- Not currently in treatment for psychosis or bipolar disorder
not used EC within the last 3 months ("exclusive smokers") or used nicotine-containing EC at least
once a week for the past month and have smoked at least 5 cpd for the last 3 months ("dual users")

Interventions "We will conduct a 2-year longitudinal cohort study comprising participants who smoke exclusively CCs $(n=175)$ and dual users of e-cigs and CCs $(n=275) "$

Outcomes $\quad$ "We will use state-of-the-art ecological momentary assessments to determine:

1) dynamic patterns of e-cig and CC use and related outcomes (e.g. dependence, withdrawal symptoms, CC quit attempts and quitting success);

2) episodic (affective, contextual, social) and stable person-factor (lifestyle factors, demographics) variables that covary meaningfully with e-cig and CC use and related outcomes;

3) biomarkers of tobacco and carcinogen exposure as well as other health-related outcomes (e.g. reduced pulmonary function)."

\begin{tabular}{ll}
\hline Starting date & September 2015 \\
\hline Contact information & PI Megan Piper \\
\hline Notes & \\
\hline
\end{tabular}


NCT02590393

\begin{tabular}{|c|c|}
\hline Study name & The role of nicotine and non-nicotine alkaloids in e-cigarette use and dependence \\
\hline \multirow[t]{3}{*}{ Methods } & Randomized parallel-assignment double-blind trial \\
\hline & Setting: Smoking research clinic, USA \\
\hline & Recruitment: volunteers \\
\hline \multirow[t]{9}{*}{ Participants } & Estimated enrolment: 375 \\
\hline & Inclusion criteria: \\
\hline & - Have no known serious medical conditions \\
\hline & - Are $18-65$ years old \\
\hline & - Smoke an average of at least $10 \mathrm{cpd}$ \\
\hline & - Have smoked at least 1 cumulative year \\
\hline & - Have an expired air $\mathrm{CO}$ reading of at least $10 \mathrm{ppm}$ \\
\hline & - Are able to read and understand English \\
\hline & Exclude if: multiple, related to baseline health status \\
\hline \multirow[t]{3}{*}{ Interventions } & - Switch to standard nicotine EC use for 8 weeks \\
\hline & - Switch to ECs with same nicotine but very low non-nicotine alkaloid levels \\
\hline & - Switch to ECs with very low nicotine and non-nicotine alkaloids \\
\hline \multirow[t]{6}{*}{ Outcomes } & Primary: \\
\hline & - CO levels at 8 weeks \\
\hline & Secondary: \\
\hline & - EC use \\
\hline & - EC solution use \\
\hline & - cigarette use, at 8 weeks \\
\hline Starting date & May 2016 \\
\hline Contact information & Jed Rose \\
\hline Notes & $\begin{array}{l}\text { "This is not a smoking cessation study; People who smoke will not be asked to quit smoking, and e- } \\
\text { cigarettes will not be used as a medical device }\end{array}$ \\
\hline & or therapy." \\
\hline
\end{tabular}

NCT02635620

\begin{tabular}{ll} 
Study name & $\begin{array}{l}\text { Changes in lung function parameters, bronchial reactivity, state of health and smoking behavior as- } \\
\text { sociated with changing from conventional } \\
\text { smoking to electronic cigarettes }\end{array}$ \\
\hline Methods & Prospective observational study \\
Setting: Community, Germany \\
Recruitment: Vape shops and smoking cessation clinics
\end{tabular}


NCT02635620 (Continued)

Participants
Estimated enrolment: 80

Inclusion criteria:

- Smoking $\geq 5$ years

- Smoking $\geq 10 \mathrm{cpd}$

- No intention to stop smoking within the last 3 months

- Using EC with nicotine

- No infection of airways at the time of measurements

- EC group: intending to use EC

- Control group: smoking cessation in the framework of a clinical conducted program

Exclude if:

- pregnancy or breastfeeding

- not speaking German

- known allergy

- acute psychiatric diseases, suicidal tendency

- drug/substance/alcohol abuse

- severe internal diseases
Interventions

Comparison between:

- People who smoke who intend to start EC use for the first time

- 2) People who smoke who intend to quit smoking within a clinical conducted smoking cessation program

\begin{tabular}{ll}
\hline Outcomes & Primary: \\
& $\cdot$ Lung function \\
& $\cdot$ QoL \\
\hline Starting date & Respiratory tract inflammation \\
\hline Contact information & October 2015 \\
\hline Notes & Tobias Rüther \\
\hline
\end{tabular}

\section{NCT03589989}

Study name

The ESTxENDS Trial- Electronic Nicotine Delivery Systems (ENDS/Vaporizer/E-cigarette) as an aid for smoking cessation. (ESTxENDS)

\begin{tabular}{ll} 
Methods & Randomized, parallel-assignment, open-label trial \\
Setting: Switzerland & Recruitment: Not specified \\
\hline Participants & Estimated Enrolment: 1172 \\
Inclusion criteria: & Informed consent as documented by signature \\
- Persons aged 18 or older \\
- Currently smoking 5 or more cigarettes a day for at least 12 months
\end{tabular}

Electronic cigarettes for smoking cessation (Review) 
- Persons providing a valid phone number, a valid email address and/or a valid postal address.

Exclusion criteria:

- Known hypersensitivity or allergy to contents of the e-liquid

- Participation in another study with investigational drug within the 30 days preceding the baseline visit and during the present study where interactions are to be expected

- Women who are pregnant or breastfeeding

- Intention to become pregnant during the course of the scheduled study intervention, i.e. within the first 6 months of the study

- Persons having used ENDS regularly in the 3 months preceding the baseline visit

- Persons having used nicotine replacement therapy (NRT) or other medications with demonstrated efficacy as an aid for smoking cessation such as varenicline or bupropion within the 3 months preceding the baseline visit

- Persons who cannot attend the 6-month follow-up visit for any reason

- Cannot understand instructions delivered in person or by phone, or otherwise unable to participate in study procedures

Interventions
- a) ENDS (vaporizer/e-cig) and smoking cessation counseling will receive:

- ENDS and nicotine-containing e-liquids, which they will be allowed to use ad libitum

- Smoking cessation counseling: provided in person at the first clinical visit and then over the phone at the target quit date 1 week later and again at weeks 2, 4 and 8 after the target quit date. After 6 months, participants will be asked to come to a final clinical visit

- Participants will be allowed to additionally use nicotine replacement therapy

- b) Control group will receive smoking cessation counseling only as provided for a). Participants will be allowed to additionally use nicotine replacement therapy

- Self-report of having smoked no cigarettes from quit date, validated by urinary levels of anabasine. If anabasine is missing, validation by exhaled carbon monoxide (CO).

Seconday outcomes:

- Continuous smoking abstinence at 6 months post-quit date

* Self-report of having smoked no cigarettes from quit date, validated by urinary levels of NNAL (4-(methylnitrosamino)-1-(3-pyridyl)-1-butanol). If NNAL is missing, validation by urinary levels of anabasine or exhaled carbon monoxide (CO)

- Self-reported smoking abstinence allowing a 2-week 'grace period' at 4, 8 weeks and 6 months post quit date

- Validated smoking abstinence allowing a 2-week ' grace period at 6 months post quit date

* validated by urinary levels of anabasine. If anabasine is missing validation by exhaled CO

* validated by urinary levels of NNAL (4-(methylnitrosamino)-1-(3-pyridyl)-1-butanol). If NNAL is missing, validation by urinary levels of anabasine or exhaled $\mathrm{CO}$

- Self-reported smoking abstinence allowing up to 5 cigarettes at 1, 2, 4, 8 weeks and 6 months post-quit date

- Validated smoking abstinence allowing up to 5 cigarettes at 6 months post-quit date:

* validated by urinary levels of anabasine. If anabasine is missing validation by exhaled CO

* validated by urinary levels of NNAL (4-(methylnitrosamino)-1-(3-pyridyl)-1-butanol). If NNAL is missing, validation by urinary levels of anabasine or exhaled $\mathrm{CO}$

- Self-reported 7-day PPA at 1, 2, 4, 8 weeks and 6 months post-quit date

- Validated 7-day PPA at 6 months post-quit date

* Confirmation of having smoked no cigarettes in the past 7 days, validated by urinary levels of anabasine. If anabasine is missing validation by exhaled $\mathrm{CO}$

* Confirmation of having smoked no cigarettes in the past 7 days, validated by urinary levels of NNAL (4-(methylnitrosamino)-1-(3-pyridyl)-1-butanol). If NNAL is missing, validation by urinary levels of anabasine or exhaled $\mathrm{CO}$ 
- Number of cpd at baseline, target quit date, 1, 2, 4, 8 weeks and 6 months post-quit date, selfreported

- Change in number of cpd at baseline, 6 months post-quit date, self-reported. Successful reduction defined as $50 \%$ reduction in cpd

- Use of any other smoking cessation products (NRT) at 1, 2, 4, 8 weeks and 6 months post-quit date, self-reported

- Withdrawal at baseline and 6 months

- Fagerström Test for Nicotine Dependence at baseline and 6 months

- Swiss EQ-5D at baseline and 6 months

- Use of any ENDS at 1, 2, 4, 8 weeks and 6 months post-quit date, self-reported

- Most common adverse events using ENDS at 1, 2, 4, 8 weeks and 6 months post-quit date

Starting date 16 July 2018

Contact information Reto Auer, reto.auer@biham.unibe.ch

Anna Schöni, anna.schoeni@biham.unibe.ch

Notes

Linked trials: NCT03603340; NCT03603353; NCT03612336; NCT03612375; NCT03612453;

NCT03612544; NCT03632421; NCT03938298

\section{NCT03700112}

Study name

An open-label, randomized cross-over study comparing nicotine pharmacokinetics of seven electronic cigarette products and one traditional cigarette across two delivery (10 puff and ad-libitum) conditions, in healthy adult smokers.

Methods

Open-label, randomized cross-over trial

Setting and recruitment not specified, New Zealand

Participants

Estimated enrolment: 24

Inclusion criteria:

- Male or female aged 18 to 60 years of age inclusive

- BMI between 18 to $35 \mathrm{~kg} / \mathrm{m}^{2}$ inclusive

- Healthy based on medical history and screening assessments, in the opinion of the Investigator

- Current smoker of at least 8 cigarettes per day on average

- Has been smoking for at least 12 months prior to screening. Brief periods of non-smoking (e.g. up to $\sim 7$ consecutive days due to illness, trying to quit, participation in a study where smoking was prohibited) are permitted at the discretion of the Investigator

- Able to participate, and willing to give written informed consent and comply with study restrictions

Exclusion criteria:

- Clinically-relevant medical or psychiatric disorder, in the opinion of the Investigator

- Clinically-significant abnormality on screening ECG

- Sustained blood pressure recordings at screening of $<90 \mathrm{mmHg}$ or $>150 \mathrm{mmHg}$ for systolic blood pressure, or $<50 \mathrm{mmHg}$ or $>90 \mathrm{mmHg}$ for diastolic blood pressure

- Sustained resting heart rate of $>100$ or $<40$ beats per minute at screening

- Positive result for urine drugs of abuse test or alcohol breath test at screening. If a positive urine drug test is observed, and it is believed the positive urine test is due to prescription drugs, the $\mathrm{PI}$ should obtain documentation that a) confirms the person's use of the prescribed medication, and b) the prescribed medication will cause a false positive drug test 
NCT03700112 (Continued)

- Clinically-significant abnormality in laboratory test results at screening, in the opinion of the Investigator

- Exposure to an investigational drug in a clinical trial within 1 month prior to Assessment Day 1

- Blood or plasma donation of $>500 \mathrm{~mL}$ within 1 month prior to Assessment Day 1

- Positive urine pregnancy test at screening or Assessment Day 1 in women

- Any clinically-significant concomitant disease or condition that could interfere with, or for which the treatment of might interfere with, the conduct of the study, or that would, in the opinion of the investigator, pose an unacceptable risk to the participant in this study

Interventions

- JUUL Virginia Tobacco flavored 5.0\% ENDS; consumed using 10 puffs delivery method, ad-libitum

- PMI iQOS Heat sticks - Regular consumed using 10 puffs delivery method, ad-libitum

- Reynolds VUSE Solo ENDS - Original consumed using 10 puffs delivery method, ad-libitum

- Imperial MyBlu ENDS - Original consumed using 10 puffs delivery method, ad-libitum

- Altria MarkTen ENDS - Bold Classic consuming using 10 puffs delivery method, ad-libitum

- MLV PHIX ENDS - Original Tobacco consumed using 10 puffs delivery method, ad-libitum

- NJOY Daily EXTRA ENDS - Rich Tobacco consumed using 10 puffs delivery method, ad-libitum

- Altria Marlboro combustible cigarette - Red consumed using 10 puffs delivery method, ad-libitum

Day 48
Outcomes:
- Nicotine PK parameters calculated from the individual plasma concentrations
- Exhaled CO
- Level of user satisfaction measured by Modified Product Evaluation Scale
- Characterize consumption of $8 \times$ E-cigarettes/cigarettes products by collecting total number of
puffs forch e-cigarette

\begin{tabular}{ll}
\hline Starting date & 7 December 2018 \\
\hline Contact information & Study director: Concetta Carbonaro \\
& Responsible party: Juul Labs, Inc. \\
\hline
\end{tabular}

Notes

NCT03962660

Study name Harm reduction for tobacco smoking with support of tobacco-replacing electronic nicotine delivery systems (HaRTS-TRENDS)

Methods

Parallel, randomized controlled trial

Setting: USA

Recruitment: from prominent Housing First programs serving chronically homeless people who are often multiply affected by psychiatric, medical and substance-use disorders. The proposed sample will be recruited from a highly vulnerable and marginalized population in a tight-knit urban community

Participants

Estimated enrolment: 94

Inclusion criteria:

- Having a history of chronic homelessness according to the widely-accepted federal definition 
NCT03962660 (Continued)

Interventions
- Being a current DESC client living in 1 of DESC's participating permanent supportive housing projects

- Being between 21 - 65 years of age

- Being a daily smoker ( $>4$ cigarettes/day in the past year with a breath $\mathrm{CO} \geq 6 \mathrm{ppm}$ or salivary cotinine test at level 1 if $\mathrm{CO}<6 \mathrm{ppm}$ )

- Having adequate English language skills to understand verbal information and communicate in the study

Exclusion Criteria:

- Use of other tobacco products besides cigarettes $\geq 9$ days in the past month

- Refusal or inability to consent to participation in research

- Constituting a risk to the safety and security of other clients or staff.

- Intervention: HaRTS-TRENDS: 4 individual sessions delivered in the context of the interventionist's pragmatic harm-reduction mindset paired with a compassionate, advocacy-oriented "heartset" or style. It comprises the delivery of 4 manualized components, including

* a) participant-led tracking of preferred smoking outcomes,

* b) elicitation of participants' harm-reduction goals and their progress toward achieving them,

* c) discussion of the relative risks of various nicotine delivery systems,

* d) instruction in using ENDS. Additionally, HaRTS-TRENDS entails provision of commercially available ENDS.

- Standard care: The 4-session, individual standard care control condition entails the well-documented and evidence-based 5 As intervention (i.e. Ask about nicotine use, Assess use, Advise to quit smoking, Assist with exploring current smoking/planning smoking cessation, Arrange follow-up). Part of arranging follow-up is the recommendation to call the smoking quit line, which can supply additional counseling and nicotine replacement therapy

Outcomes

Primary outcomes, measured across the 12-month follow-up:

- Biologically-verified nonsmoking (i.e. self-reported nonsmoking if corresponding CO measure is $<8$ ) in the past 7 days

- Urinary concentration of a tobacco-specific nitrosamine

Secondary outcomes, measured across the 12-month follow-up:

- Self-reported smoking intensity is the mean number of cigarettes participants report smoking per day in the 7 days prior to the assessment

- Self-reported smoking frequency is the number of days participants report smoking in the 7 days prior to the assessment

- CO level

- Urinary cotinine

- FEV $1 \%$

- 10-item Clinical COPD Questionnaire

- EQ-5D-5L

Other outcomes:

- Smoking craving

- Side effects of ENDS
9 May 2019

Tatiana M Ubay, tatiubay@uw.edu

Tatiana MUbay, tatiubay@uw.edu

Notes 
NCT04063267

\begin{tabular}{|c|c|}
\hline Study name & Electronic cigarettes as a harm reduction strategy in individuals with substance use disorder \\
\hline Methods & $\begin{array}{l}\text { Parallel, randomized trial } \\
\text { Recruitment/Setting: Not specified }\end{array}$ \\
\hline Participants & $\begin{array}{l}\text { Estimated enrolment: } 240 \\
\text { Inclusion criteria: } \\
\text { - Smokes at least } 10 \mathrm{cpd} \\
\text { - Meet DSM-V AUD and/or OUD within the past year, interested in reducing cpd } \\
\text { - Able to provide consent } \\
\text { - Use a cell phone, are willing/able to receive and respond to daily text messages about their ciga- } \\
\text { rette use and e-cigarette use on their cell phone } \\
\text { - Provide } 1 \text { additional contact, and are willing to use an e-cigarette for } 3 \text { weeks } \\
\text { Exclusion criteria: } \\
\text { - Pregnant and/or breast feeding (self-reported) } \\
\text { - Currently using smoking cessation medications (including other forms of NRT, bupropion, or } \\
\text { varenicline) } \\
\text { - enrolled in a smoking cessation program or another cessation trial } \\
\text { - Have used an e-cigarette in the past } 14 \text { days } \\
\text { - Have used any other tobacco products (pipe, cigar, cigarillos, snuff, chewing tobacco, rolling to- } \\
\text { bacco, or hookah/shisha) in the past } 30 \text { days } \\
\text { - Report having a history of asthma, other airways diseases, or heart disease }\end{array}$ \\
\hline
\end{tabular}

Interventions

E-cigarettes arm:

- Participants will be encouraged to substitute e-cigarettes for combustible cigarettes in order to reduce nicotine withdrawal symptoms

\section{Nicotine Replacement Therapy arm:}

- Nicotine patches and gum to last them the first week based on their baseline recorded smoking. Participants will be advised to use both a $21 \mathrm{mg}$ nicotine patch and $4 \mathrm{mg}$ nicotine for cravings

Outcomes $\quad$ Proportion of participants who achieve $50 \%$ reduction in cpd at 3 weeks

Starting date $\quad 15$ September 2019

Contact information NYU Langone Health, Scott.Sherman@nyulangone.org

Notes

Study name A randomized controlled international multicentre study evaluating changes in metabolic syndrome in smokers with type 2 diabetes mellitus after switching from tobacco cigarettes to combustion-free nicotine delivery systems: DIASMOKE Study

Short title: Metabolic syndrome in diabetic smokers using cigarettes \& combustion-free nicotine delivery systems (DIASMOKE) 
NCT04231838 (Continued)

Methods

RCT

Setting: Italy
576 participants

Inclusion criteria:

Participants will be required to satisfy all of the following criteria at the screening visit, unless otherwise stated:

- Participants will be: 1.1 . over 23 years of age

- T2DM Patients will have: 2.1. body mass index (BMI) between 17.6 and $32.0 \mathrm{~kg} / \mathrm{m}^{2}$, inclusive 2.2. body weight exceeding $50 \mathrm{~kg}$ (men) or $40 \mathrm{~kg}$ women $2.36 .5<\mathrm{HbA} 1 \mathrm{C}<103.2$. completion of proforma (CRF) 3.3. lab assessment as outlined in the CRF

- Participants will be willing to refrain from eating/drinking prior to screening and check-in at each study visit.

- Participants will be regular smokers of at least 10 cigarettes/day (max 30 cigarette/day)

- Participants will have smoked for at least 5 consecutive years prior to screening

- Participants must have a saliva cotinine level $>10 \mathrm{ng} / \mathrm{mL}$ or an exhaled breath CO (eCO) level > 7 ppm at screening

- Participants in Arm A who continue to smoke will be willing to use their own brand/type cigarettes

- Participants in Arm B will be willing to use the study products (THP product or e-cigarette) provided to them during the study

Exclusion Criteria:

Participants will be excluded at the screening visit based on the following criteria:

- Women who are pregnant or breastfeeding. This will be confirmed at screening and at visit 1. Any woman who becomes pregnant during this study will be withdrawn

- People with a history of recent acute decompensation of their disease requiring treatment within 4 weeks prior to visit 1

- People who have a significant history of alcoholism or drug/chemical abuse within 24 months prior to screening, as determined by the investigator

- People who are still participating in another clinical study (e.g. attending follow-up visits) or who have participated in a clinical study involving administration of an investigational drug (new chemical entity) in the past 3 months prior to first product use

- People who have, or who have a history of, any clinically-significant neurological, gastrointestinal, renal, hepatic, cardiovascular, psychiatric, respiratory, metabolic, endocrine, hematological or other major disorder that, in the opinion of the investigator or their appropriately qualified designee, would jeopardize the safety of the person or impact on the validity of the study results

- People who regularly use any nicotine (e.g. e-cigarettes, NRT) or tobacco product (e.g. HTPs, oral smokeless) other than their own cigarettes within 14 days of screening

At screening and prior to enrolment, all patients will be offered a locally-available free smoking cessation program as per local guidelines. Those who express the intention of booking for the cessation program together with those who, at screening, are planning to quit smoking in the next 6 months, will not be recruited into the study. Patients taking part in the study will be informed that they are free to quit smoking and withdraw from the study at any time. Any person who decides to quit smoking will be directed to local stop smoking services.

Interventions Arm A: tobacco cigarettes (continuing smoking their own tobacco cigarette brand)

Arm B: switching to using combustion-free nicotine delivery systems (C-F NDS)
Time frame: 3 months, 6 months, 1 year and 2 years 
NCT04231838 (Continued)

Change in metabolic syndrome prevalence

Change in plasma glucose

Change in triglycerides

Change in high-density lipoprotein (HDL)

Change in waist circumference

Starting date

Contact information

Estimated start date: 17 September 2020. Estimated primary completion September 2021. Estimated study completion March 2025

Daniela Saitta, PhD, daniela.saitta@eclatrbc.it

Riccardo Polosa, PhD, polosa@unict.it

\section{NCT04238832}

Study name

Impact of non-cigarette tobacco product formulation on reinforcement value and use in current smokers

Short title: Salt-Based E-cigarette

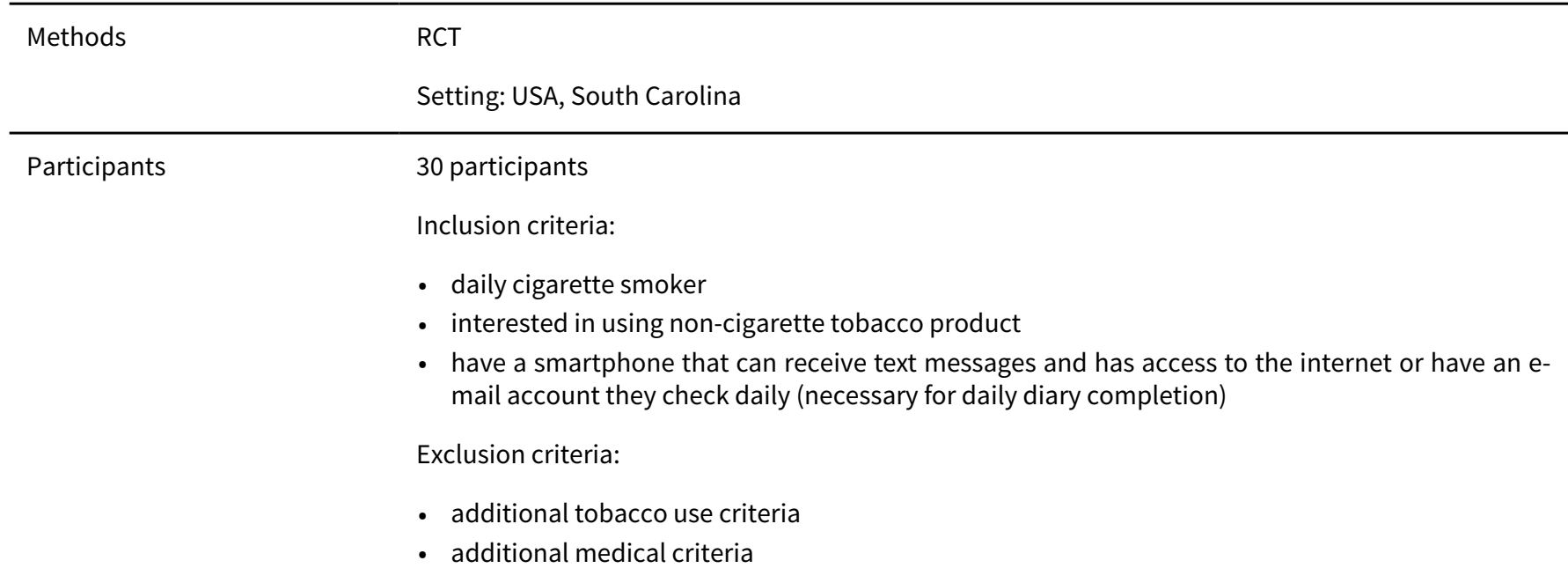

Salt base nicotine

Free base nicotine

\section{Outcomes}

Most preferred product [ Time Frame: Lab Visit 2, occurring approximately 1 week after the initial screening/baseline visit ]

Participants complete a preference assessment in which they choose between the salt liquid, freebase liquid, or a traditional cigarette in a series of trials. The outcome of this assessment is the product chosen most often by each participant

Cigarettes per day [ Time Frame: Week 2 of study ]

The average number of cigarettes smoked per day during the 1 week sampling period.

Biomarkers (i.e. expired CO, cotinine) will corroborate self-reported indices of use 
NCT04238832 (Continued)

Starting date

Contact information
23 June 2020. Estimated completion August 2021

Notes \\ Study name}

Official title: Cigarette consumption after switching to high or low Nicotine strENght E-cigaretteS In Smokers with Schizophrenia spectrum disorders: a 12-month randomized, double-blind multicentre trial

Brief title: Cigarette consumption after switchinG to high or low nicotine strENght E-cigaretteS In Smokers with Schizophrenia (GENESIS)

\begin{tabular}{ll}
\hline Methods & RCT \\
Multicenter: Italy, Russia, Ukraine, UK \\
Collaborators: \\
- Juul Labs, Inc. \\
- St. Petersburg State Pavlov Medical University \\
- Bashkir State Medical University \\
- Ukrainian Institute on Public Health Policy \\
- University of Surrey \\
- Eclat Srl
\end{tabular}

Participants

Estimated enrolment: 260

Inclusion criteria:

- $\operatorname{Adult}(>18$ yrs)

- Regular smoking (> 10 cigarettes a day; for at least 1 year)

- Exhaled breath CO (eCO) level > 7 ppm

- Not currently attempting to quit smoking or wishing to do so in the next 30 days; this will be verified at screening by the answer "NO" to the question "Do you intend to quit in the next 30 days?"

- Schizophrenia spectrum disorder diagnosis (schizophrenia, delusional disorder, schizoaffective disorder, personality disorder, schizoid personality disorder, etc) by DSM-V criteria

- Understand and provide informed consent

- Able to comply with all study procedures

Exclusion criteria:

- Institutionalized patients

- Acute decompensation of Schizophrenia spectrum disorder symptoms within the past month

- Change in antipsychotic treatment within the past month

- No recent history of hospitalization for any serious medical condition within 3 months prior to screening, as determined by the investigator

- Myocardial infarction or angina pectoris within 3 months prior to screening, as determined by the investigator

- Current poorly-controlled asthma or COPD

- Pregnancy, planned pregnancy or breastfeeding. Any female participant who becomes pregnant during this study will be withdrawn

- People who have a significant history of alcoholism or drug/chemical abuse within 12 months prior to screening, as determined by the investigator. 
NCT04452175 (Continued)

- Accepting to take part in a smoking cessation program

- People who regularly use any recreational nicotine (e.g. e-cigarettes,) or tobacco product (e.g. tobacco heated products, oral smokeless) other than their own cigarettes within 30 days of screening

- People who have used smoking cessation therapies (e.g. varenicline, bupropion, or NRT) within 30 days of screening

- People who are still participating in another clinical study (e.g. attending follow-up visits) or who have recently participated in a clinical study involving administration of an investigational drug (new chemical entity) within the past 3 months

- People who have, or who have a history of, any clinically-significant neurological, gastrointestinal, renal, hepatic, cardiovascular, psychiatric, respiratory, metabolic, endocrine, hematological or other major disorder that, in the opinion of the investigator or their appropriately qualified designee, would jeopardize the safety of the participant or impact on the validity of the study results

$\begin{array}{ll}\text { Interventions } & \text { Experimental: HIGH 5\%. Intervention: JUUL E-CIGARETTE } \\ & \text { - Active Comparator: LOW 1.7\%. Intervention: JUUL E-CIGARETTE }\end{array}$

\section{Outcomes}

Primary outcomes:

- Rates of participants with continuous smoking abstinence at 6 months; Time Frame: 24 weeks

- Self-reported continuous smoking abstinence at 6 months from the previous visit, biochemically-verified by exhaled CO measurements of $\leq 7 \mathrm{ppm}$

Secondary outcomes

- Rates of participants with continuous smoking abstinence at 12-month [ Time Frame: 52 weeks ]

- Rates of participants with continuous smoking reduction at 6-month [ Time Frame: 24 weeks]

- Rates of participants with continuous smoking reduction at 12-month [ Time Frame: 52 weeks]

- Proportion of AEs [ Time Frame: 24 weeks]

- Absolute change in PANSS [ Time Frame: 24 weeks]

- Absolute change in mCEQ [ Time Frame: 24 weeks]

- Absolute change in Chester Step Test-derived values [ Time Frame: 24 weeks]

- Change in App-derived endpoints (self-rated mental health -SRMH). [ Time Frame: 24 weeks]

Starting date October 2020. Estimated completion date March 2022

Contact information

Pasquale Caponnetto, p.caponnetto@unict.it

\section{NCT04649645}

Study name International randomized controlled trial evaluating changes in oral health in smokers after switching to combustion-free nicotine delivery systems (SMILE)

Methods RCT

Setting: multicenter: Italy, Moldova, Poland, UK and Indonesia

Participants

Estimated enrolment 606 participants

Inclusion criteria:

- Demonstrate understanding of the study and willingness to participate in the study by providing a signed written informed consent

- Healthy, not taking regular medications for chronic medical conditions 
- Adults, age at least 18 years old

- Presence of at least 10 natural anterior teeth in total (cuspid to cuspid, lower and upper jaw)

- Presence of at least 18 'scorable' teeth with scorable facial and lingual surfaces. Teeth that are grossly carious, orthodontically banded, exhibiting general cervical abrasion and/or enamel abrasion, and third molars will not be included in the tooth count

- Willingness and ability to comply with the requirements of the study including installing an APP on their digital device, e.g. smart phone or tablet

For Arms A and B, participants have to be:

- Regular smokers, defined as: smoked for at least 5 consecutive years prior to screening. Smoked $>10$ and $<30$ cigarettes per day (cpd). with an exhaled breath carbon monoxide (CO) level $\geq 7 \mathrm{ppm}$ at screening

- willing to regularly use any nicotine or tobacco product other than their own conventional cigarettes brand within 14 days prior to screening

- willing to change to use of study products or if randomized to Arm A continuing to use their own brand of conventional cigarettes for the whole duration of the study

For Arm C, participants have to be:

- Never-smokers, defined as:never smoked or who have smoked $<100$ cigarettes in their lifetime and none in the 30 days prior to screening.with an exhaled breath CO level $<7 \mathrm{ppm}$ at screening

- willing to not smoke or use any form of tobacco or nicotine-containing products for the whole duration of the study

Exclusion criteria:

- Pregnancy

- Presence of extensive crown or bridge work, dental implants, and/or rampant decay (per Investigator/Examiner discretion)

- Significant oral soft tissue pathology or any type of gingival overgrowth, other than plaque-induced gingivitis and mild periodontitis (Stage I)

- Moderate-to-severe periodontitis (Stage II, III and IV) based on 2017 World Workshop on the Classification of Periodontal and Peri-Implant Diseases and Conditions, which require:Detectable Interdental Clinical Attachment Loss (CAL) $\geq 3 \mathrm{~mm}$ at $\geq 2$ non-adjacent teeth. Buccal or Oral CAL $\geq 3$ $\mathrm{mm}$ with pocketing $\geq 5 \mathrm{~mm}$ detectable at $\geq 2$ teeth

- Removable dentures or fixed and removable orthodontic appliance (except fixed lingual wires)

- Significant history of alcoholism or drug abuse (other than tobacco/nicotine) within 24 months prior to screening, as determined by the Investigator

- A course of treatment with any medications or substances (other than tobacco/nicotine) which:interfere with the cyclo-oxygenase pathway (e.g. anti-inflammatory drugs including aspirin and ibuprofen) within 3 days prior to each visit.are known to have antibacterial activity (e.g. antibiotics) within 7 days prior to each visit

Interventions

Standard Arm (Arm A): own tobacco cigarette brand

Intervention Arm (Arm B): combustion-free nicotine delivery system (C-F NDS)

Control Arm (Arm C): no smoking or use of any nicotine/tobacco products

Outcomes

Oral health parameters and teeth appearance, comparing short- and long-term impact on periodontal health between smokers continuing with conventional cigarette smoking, those switching to combustion-free nicotine delivery systems (C-F NDS), and never-smokers over 18 months

Starting date

Not yet recruiting (last updated February 2021)

Estimated study start date Feb 2021. Primary completion date Feb 2023. Completion April 2023 
NCT04649645 (Continued)

info@addendo.net

Notes

BMI: body mass index; CAR: continuous abstinence rate; CO: carbon monoxide; COPD: chronic obstructive pulmonary disease; cpd: cigarettes per day; CVD: cardiovascular disease; EC: electronic cigarette; ECG: electrocardiogram; FTND: Fagerström Test for Nicotine Dependence; NNAL: carcinogen found in tobacco smoke; NRT: nicotine replacement therapy; PP(A): point prevalence (abstinence); QoL: quality of life; TQD: target quit date; wk: week; yr: year

\section{DATA AND ANALYSES}

\section{Comparison 1. Nicotine EC versus NRT}

\begin{tabular}{|c|c|c|c|c|}
\hline $\begin{array}{l}\text { Outcome or subgroup } \\
\text { title }\end{array}$ & No. of studies & $\begin{array}{l}\text { No. of partici- } \\
\text { pants }\end{array}$ & Statistical method & Effect size \\
\hline 1.1 Smoking cessation & 3 & 1498 & Risk Ratio (M-H, Fixed, 95\% Cl) & $1.69[1.25,2.27]$ \\
\hline 1.2 Adverse events & 2 & 485 & Risk Ratio (M-H, Fixed, 95\% Cl) & $0.98[0.80,1.19]$ \\
\hline 1.2.1 4 weeks & 1 & 29 & Risk Ratio (M-H, Fixed, 95\% Cl) & $0.74[0.31,1.73]$ \\
\hline 1.2.2 6 months & 1 & 456 & Risk Ratio (M-H, Fixed, 95\% Cl) & $0.99[0.81,1.22]$ \\
\hline $\begin{array}{l}1.3 \text { Serious adverse } \\
\text { events }\end{array}$ & 2 & 727 & Risk Ratio (M-H, Fixed, 95\% Cl) & $1.37[0.77,2.41]$ \\
\hline 1.3.1 4 weeks & 1 & 29 & Risk Ratio (M-H, Fixed, 95\% Cl) & Not estimable \\
\hline 1.3.2 1 year & 1 & 698 & Risk Ratio (M-H, Fixed, 95\% Cl) & $1.37[0.77,2.41]$ \\
\hline $\begin{array}{l}\text { 1.4 Carbon monoxide } \\
(\mathrm{ppm})\end{array}$ & 2 & & Mean Difference (IV, Fixed, 95\% CI) & Subtotals only \\
\hline 1.4.1 8 weeks & 2 & 136 & Mean Difference (IV, Fixed, 95\% CI) & $-0.66[-1.94,0.62]$ \\
\hline 1.5 Heart rate (bpm) & 1 & & Mean Difference (IV, Fixed, 95\% CI) & Totals not selected \\
\hline 1.5.1 8 weeks & 1 & & Mean Difference (IV, Fixed, 95\% CI) & Totals not selected \\
\hline $\begin{array}{l}1.6 \text { Systolic blood pres- } \\
\text { sure }\end{array}$ & 1 & & Mean Difference (IV, Fixed, 95\% CI) & Totals not selected \\
\hline 1.6.1 8 weeks & 1 & & Mean Difference (IV, Fixed, 95\% CI) & Totals not selected \\
\hline $\begin{array}{l}1.7 \text { Blood oxygen satu- } \\
\text { ration }\end{array}$ & 1 & & Mean Difference (IV, Fixed, 95\% CI) & Totals not selected \\
\hline 1.7.1 8 weeks & 1 & & Mean Difference (IV, Fixed, 95\% CI) & Totals not selected \\
\hline $\begin{array}{l}1.83-\mathrm{HPMA} \text { (pmol/mg } \\
\text { creatinine) }\end{array}$ & 1 & & Mean Difference (IV, Fixed, 95\% CI) & Totals not selected \\
\hline 1.8.1 8 weeks & 1 & & Mean Difference (IV, Fixed, 95\% CI) & Totals not selected \\
\hline
\end{tabular}




\begin{tabular}{|c|c|c|c|c|}
\hline $\begin{array}{l}\text { Outcome or subgroup } \\
\text { title }\end{array}$ & No. of studies & $\begin{array}{l}\text { No. of partici- } \\
\text { pants }\end{array}$ & Statistical method & Effect size \\
\hline $\begin{array}{l}1.9 \text { NNAL (pmol/mg cre- } \\
\text { atinine)) }\end{array}$ & 1 & & Mean Difference (IV, Fixed, 95\% CI) & Totals not selected \\
\hline 1.9.1 8 weeks & 1 & & Mean Difference (IV, Fixed, 95\% CI) & Totals not selected \\
\hline $\begin{array}{l}1.102-\mathrm{HPMA} \text { (pmol/mg } \\
\text { creatinine) }\end{array}$ & 1 & & Mean Difference (IV, Fixed, 95\% CI) & Totals not selected \\
\hline 1.10.18 weeks & 1 & & Mean Difference (IV, Fixed, 95\% CI) & Totals not selected \\
\hline $\begin{array}{l}1.11 \text { HMPMA (pmol/mg } \\
\text { creatinine) }\end{array}$ & 1 & & Mean Difference (IV, Fixed, 95\% CI) & Totals not selected \\
\hline 1.11.1 8 weeks & 1 & & Mean Difference (IV, Fixed, 95\% CI) & Totals not selected \\
\hline $\begin{array}{l}1.12 \text { PheT (pmol/mg } \\
\text { creatinine) }\end{array}$ & 1 & & Mean Difference (IV, Fixed, 95\% CI) & Totals not selected \\
\hline 1.12.18 weeks & 1 & & Mean Difference (IV, Fixed, 95\% CI) & Totals not selected \\
\hline $\begin{array}{l}1.13 \text { CEMA (pmol/mg } \\
\text { creatinine) }\end{array}$ & 1 & & Mean Difference (IV, Fixed, 95\% CI) & Totals not selected \\
\hline 1.13.1 8 weeks & 1 & & Mean Difference (IV, Fixed, 95\% CI) & Totals not selected \\
\hline $\begin{array}{l}1.14 \text { AAMA (pmol/mg } \\
\text { creatinine) }\end{array}$ & 1 & & Mean Difference (IV, Fixed, 95\% CI) & Totals not selected \\
\hline 1.14.18 weeks & 1 & & Mean Difference (IV, Fixed, 95\% CI) & Totals not selected \\
\hline 1.15 FEV1 (ml) & 1 & & Mean Difference (IV, Fixed, 95\% CI) & Totals not selected \\
\hline 1.15.1 8 weeks & 1 & & Mean Difference (IV, Fixed, 95\% CI) & Totals not selected \\
\hline 1.16 FEV1/FVC (\%) & 1 & & Mean Difference (IV, Fixed, 95\% CI) & Totals not selected \\
\hline 1.16 .18 weeks & 1 & & Mean Difference (IV, Fixed, 95\% CI) & Totals not selected \\
\hline
\end{tabular}


Analysis 1.1. Comparison 1: Nicotine EC versus NRT, Outcome 1: Smoking cessation

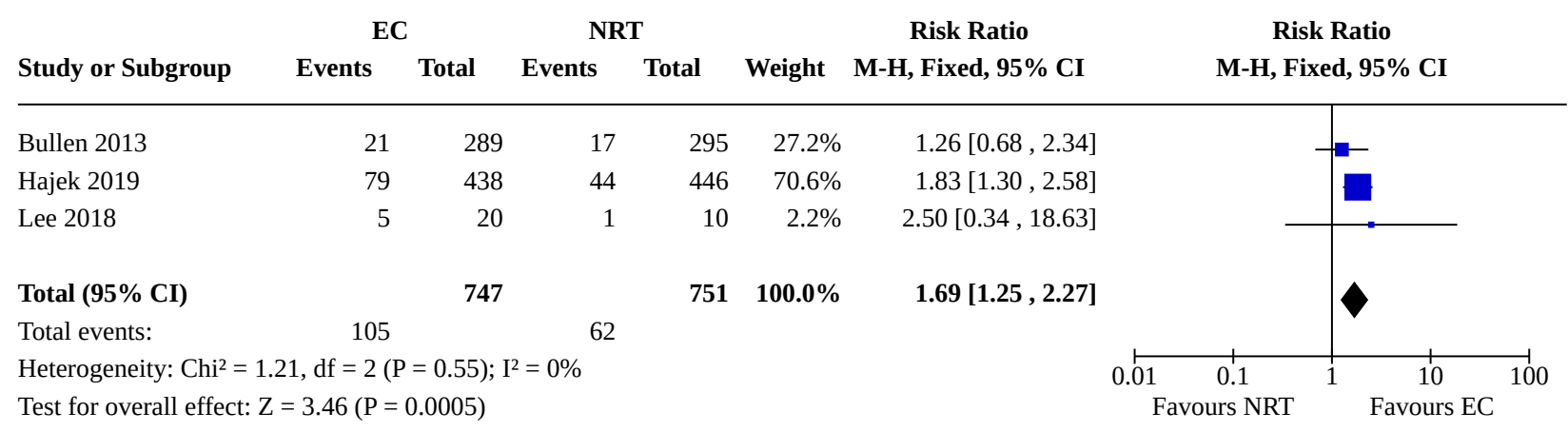

Test for subgroup differences: Not applicable

\section{Analysis 1.2. Comparison 1: Nicotine EC versus NRT, Outcome 2: Adverse events}

\begin{tabular}{|c|c|c|c|c|c|c|c|}
\hline \multirow[b]{2}{*}{ Study or Subgr } & \multicolumn{2}{|c|}{ Nicotine EC } & \multicolumn{2}{|c|}{ NRT } & \multicolumn{2}{|r|}{ Risk Ratio } & Risk Ratio \\
\hline & Events & Total & Events & Total & Weight & M-H, Fixed, 95\% CI & M-H, Fixed, 95\% CI \\
\hline
\end{tabular}

\begin{tabular}{lllllll}
\hline $\mathbf{1 . 2 . 1} 4$ weeks & & & & & & \\
Lee $2018(1)$ & 7 & 19 & 5 & 10 & $6.1 \%$ & $0.74[0.31,1.73]$ \\
Subtotal (95\% CI) & & $\mathbf{1 9}$ & & $\mathbf{1 0}$ & $\mathbf{6 . 1 \%}$ & $\mathbf{0 . 7 4}[\mathbf{0 . 3 1 , \mathbf { 1 . 7 3 }}$ \\
Total events: & 7 & & 5 & & &
\end{tabular}

Heterogeneity: Not applicable

Test for overall effect: $\mathrm{Z}=0.70(\mathrm{P}=0.48)$

$\begin{array}{lcccccc}\text { 1.2.2 } \mathbf{6} \text { months } & & & & & & \\ \text { Bullen } 2013 & 107 & 241 & 96 & 215 & 93.9 \% & 0.99[0.81,1.22] \\ \text { Subtotal (95\% CI) } & & \mathbf{2 4 1} & & \mathbf{2 1 5} & \mathbf{9 3 . 9 \%} & \mathbf{0 . 9 9}[\mathbf{0 . 8 1} \mathbf{1 . 2 2}] \\ \text { Total events: } & 107 & & 96 & & & \end{array}$

Heterogeneity: Not applicable

Test for overall effect: $\mathrm{Z}=0.05(\mathrm{P}=0.96)$

$\begin{array}{lllllll}\text { Total (95\% CI) } & & \mathbf{2 6 0} & & \mathbf{2 2 5} & \mathbf{1 0 0 . 0} \% & \mathbf{0 . 9 8}[\mathbf{0 . 8 0}, \mathbf{1 . 1 9}] \\ \text { Total events: } & 114 & & 101 & & & \end{array}$

Heterogeneity: $\mathrm{Chi}^{2}=0.45, \mathrm{df}=1(\mathrm{P}=0.50) ; \mathrm{I}^{2}=0 \%$

Test for overall effect: $\mathrm{Z}=0.21(\mathrm{P}=0.83)$

Test for subgroup differences: $\mathrm{Chi}^{2}=0.45, \mathrm{df}=1(\mathrm{P}=0.50), \mathrm{I}^{2}=0 \%$

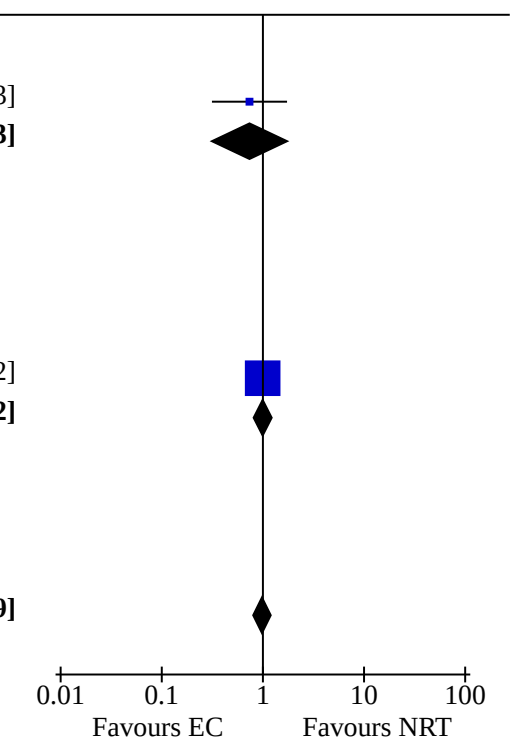

Footnotes

(1) Data at 4 weeks post-operation; time from baseline not defined and likely to differ between participants 
Analysis 1.3. Comparison 1: Nicotine EC versus NRT, Outcome 3: Serious adverse events

\begin{tabular}{|c|c|c|c|c|c|c|c|}
\hline & \multicolumn{2}{|c|}{ EC } & \multicolumn{2}{|c|}{ NRT } & \multicolumn{2}{|r|}{ Risk Ratio } & Risk Ratio \\
\hline Study or Subgroup & Events & Total & Events & Total & Weight & M-H, Fixed, 95\% CI & M-H, Fixed, 95\% CI \\
\hline
\end{tabular}

\begin{tabular}{llllll}
\hline $\mathbf{1 . 3 . 1} 4$ weeks & & & & & \\
Lee $2018(1)$ & 0 & 19 & 0 & 10 & Not estimable \\
Subtotal (95\% CI) & & $\mathbf{1 9}$ & & $\mathbf{1 0}$ & Not estimable \\
Total events: & 0 & & 0 & &
\end{tabular}

Total events:

Test for overall effect: Not applicable

\begin{tabular}{|c|c|c|c|c|c|c|}
\hline \multicolumn{7}{|l|}{ 1.3.2 1 year } \\
\hline Hajek 2019 & 27 & 356 & 19 & 342 & $100.0 \%$ & $1.37[0.77,2.41]$ \\
\hline Subtotal (95\% CI) & & 356 & & 342 & $100.0 \%$ & $1.37[0.77,2.41]$ \\
\hline Total events: & 27 & & 19 & & & \\
\hline
\end{tabular}

Heterogeneity: Not applicable

Test for overall effect: $\mathrm{Z}=1.07(\mathrm{P}=0.28)$

Total (95\% CI)

Total events:

375

Heterogeneity: Not applicable

Test for overall effect: $\mathrm{Z}=1.07(\mathrm{P}=0.28)$

Test for subgroup differences: Not applicable

\section{Footnotes}

(1) Data at 4 weeks post-operation; time from baseline not defined and likely to differ between participants

Analysis 1.4. Comparison 1: Nicotine EC versus NRT, Outcome 4: Carbon monoxide (ppm)

\begin{tabular}{|c|c|c|c|c|c|c|c|c|c|}
\hline & \multicolumn{3}{|c|}{ Nicotine EC } & \multicolumn{3}{|c|}{ NRT } & \multicolumn{2}{|r|}{ Mean Difference } & Mean Difference \\
\hline Study or Subgroup & Mean & SD & Total & Mean & SD & Total & Weight & IV, Fixed, 95\% CI & IV, Fixed, 95\% CI \\
\hline
\end{tabular}

1.4.1 8 weeks

$\begin{array}{lrrrrrrrr}\text { Hatsukami 2020 } & -9.1 & 2.9 & 58 & -8.6 & 3.9 & 52 & 98.2 \% & -0.50[-1.80,0.80] \\ \text { Lee } 2018 & -2.1 & 12.2 & 18 & 7.1 & 11 & 8 & 1.8 \% & -9.20[-18.68,0.28] \\ \text { Subtotal (95\% CI) } & & & \mathbf{7 6} & & & \mathbf{6 0} & \mathbf{1 0 0 . 0 \%} & \mathbf{- 0 . 6 6}[-\mathbf{1 . 9 4 , 0 . 6 2}]\end{array}$

Subtotal (95\% CI) 76

Heterogeneity: $\mathrm{Chi}^{2}=3.18, \mathrm{df}=1(\mathrm{P}=0.07) ; \mathrm{I}^{2}=69 \%$

Test for overall effect: $\mathrm{Z}=1.01(\mathrm{P}=0.31)$

Test for subgroup differences: Not applicable

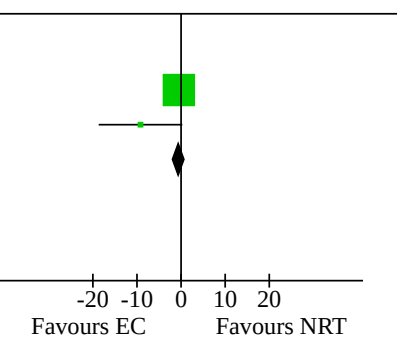

Analysis 1.5. Comparison 1: Nicotine EC versus NRT, Outcome 5: Heart rate (bpm)

\begin{tabular}{|c|c|c|c|c|c|c|c|c|}
\hline & & otine & & & NRT & & Mean Difference & Mean Difference \\
\hline Study or Subgroup & Mean & SD & Total & Mean & SD & Total & IV, Fixed, 95\% CI & IV, Fixe \\
\hline
\end{tabular}

$\begin{array}{lllll}-4.6 & 3.9 & 58 & -3.2 & 3.3\end{array}$

53

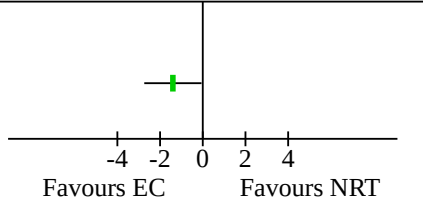


Analysis 1.6. Comparison 1: Nicotine EC versus NRT, Outcome 6: Systolic blood pressure

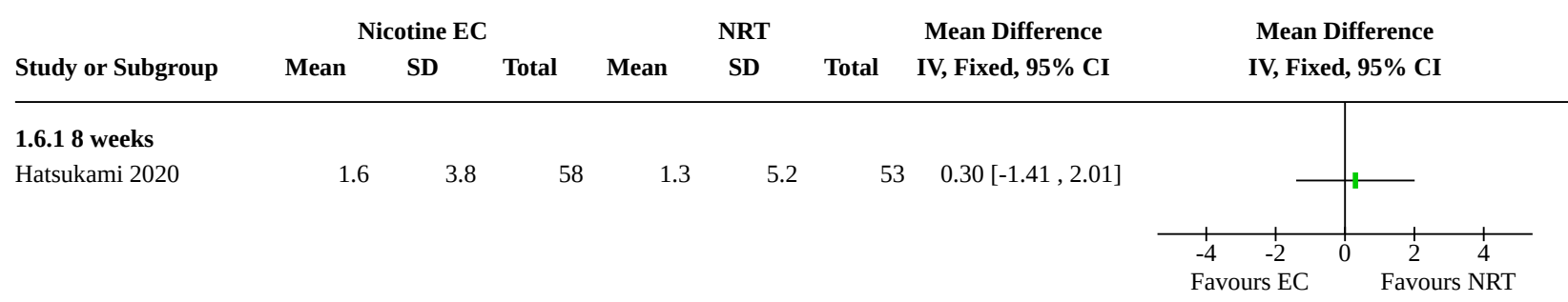

Analysis 1.7. Comparison 1: Nicotine EC versus NRT, Outcome 7: Blood oxygen saturation

\begin{tabular}{|c|c|c|c|c|c|c|c|c|}
\hline & & tine & & & NRT & & Mean Difference & Mean Difference \\
\hline Study or Subgroup & Mean & SD & Total & Mean & SD & Total & IV, Fixed, 95\% CI & IV, Fixed, 95\% CI \\
\hline
\end{tabular}

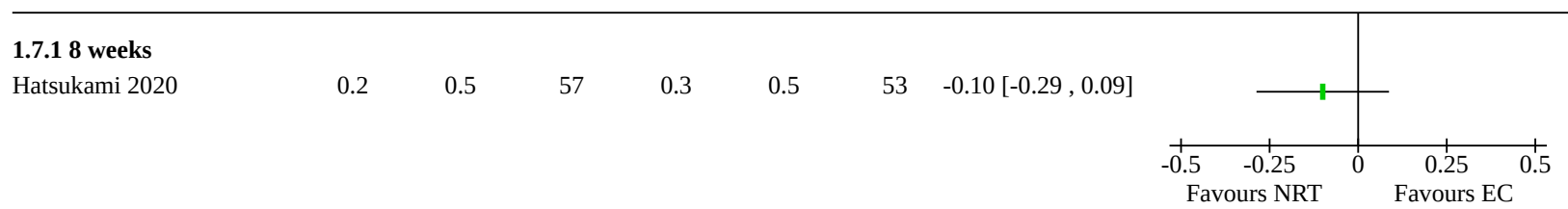

Analysis 1.8. Comparison 1: Nicotine EC versus NRT, Outcome 8: 3-HPMA (pmol/mg creatinine)

\begin{tabular}{|c|c|c|c|c|c|c|c|c|c|}
\hline \multirow[b]{2}{*}{ Study or Subgroup } & \multicolumn{3}{|c|}{ Nicotine EC } & \multicolumn{3}{|c|}{ NRT } & \multirow{2}{*}{$\begin{array}{c}\text { Mean Difference } \\
\text { IV, Fixed, 95\% CI }\end{array}$} & \multirow{2}{*}{\multicolumn{2}{|c|}{$\begin{array}{c}\text { Mean Difference } \\
\text { IV, Fixed, 95\% CI }\end{array}$}} \\
\hline & Mean & SD & Total & Mean & SD & Total & & & \\
\hline \multicolumn{10}{|l|}{ 1.8.1 8 weeks } \\
\hline Hatsukami 2020 & -2681 & 1523 & 58 & -2307 & 1788 & 53 & $-374.00[-994.76,246.76]$ & 1 & - \\
\hline & & & & & & & & $\begin{array}{cc}-1000 & -500 \\
\text { Favours EC }\end{array}$ & $\begin{array}{c}500 \\
\text { Favours NRT }\end{array}$ \\
\hline
\end{tabular}

Analysis 1.9. Comparison 1: Nicotine EC versus NRT, Outcome 9: NNAL (pmol/mg creatinine))

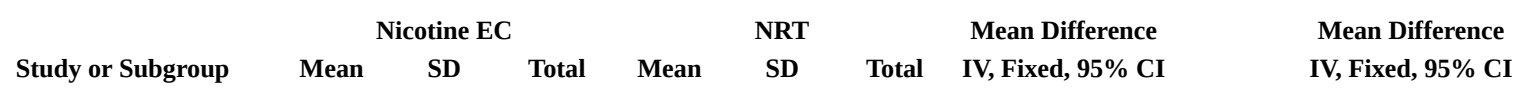

1.9.1 8 weeks

Hatsukami 2020

$\begin{array}{llll}-0.6 & 0.5 & 57 & -0.9\end{array}$

0.6

53

$0.30[0.09,0.51]$

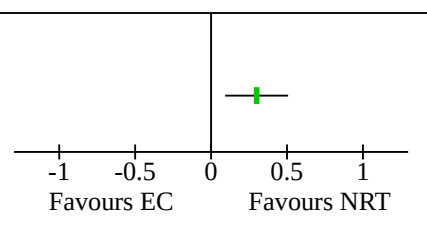

Analysis 1.10. Comparison 1: Nicotine EC versus NRT, Outcome 10: 2-HPMA (pmol/mg creatinine)

\begin{tabular}{|c|c|c|c|c|c|c|c|c|c|}
\hline \multirow[b]{2}{*}{ Study or Subgroup } & \multicolumn{3}{|c|}{ Nicotine EC } & \multicolumn{3}{|c|}{ NRT } & \multirow{2}{*}{$\begin{array}{c}\text { Mean Difference } \\
\text { IV, Fixed, 95\% CI }\end{array}$} & \multirow{2}{*}{\multicolumn{2}{|c|}{$\begin{array}{c}\text { Mean Difference } \\
\text { IV, Fixed, 95\% CI }\end{array}$}} \\
\hline & Mean & SD & Total & Mean & SD & Total & & & \\
\hline \multicolumn{10}{|l|}{ 1.10.1 8 weeks } \\
\hline \multirow[t]{2}{*}{ Hatsukami 2020} & -249 & 525 & 58 & -106 & 303 & 53 & $-143.00[-300.83,14.83]$ & -1 & \\
\hline & & & & & & & & $\begin{array}{c}-200-100 \\
\text { Favours EC }\end{array}$ & $\begin{array}{l}100200 \\
\text { Favours NRT }\end{array}$ \\
\hline
\end{tabular}


Analysis 1.11. Comparison 1: Nicotine EC versus NRT, Outcome 11: HMPMA (pmol/mg creatinine)

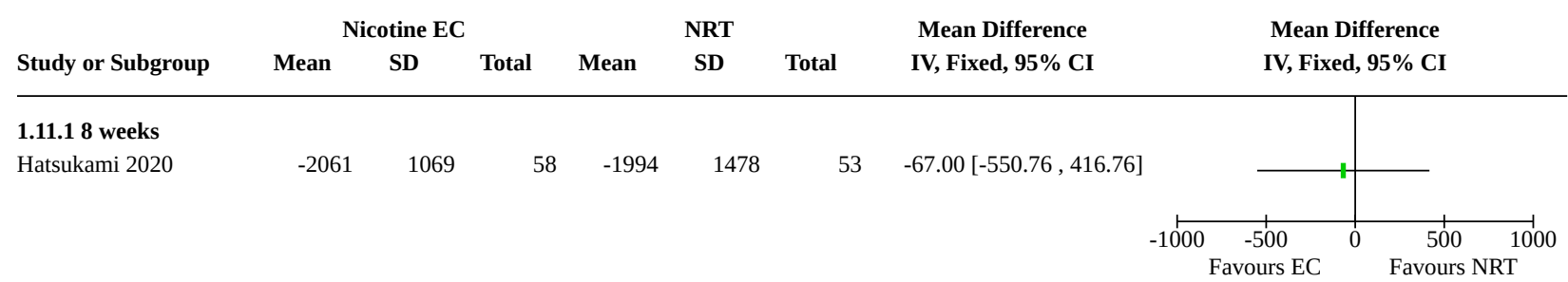

Analysis 1.12. Comparison 1: Nicotine EC versus NRT, Outcome 12: PheT (pmol/mg creatinine)

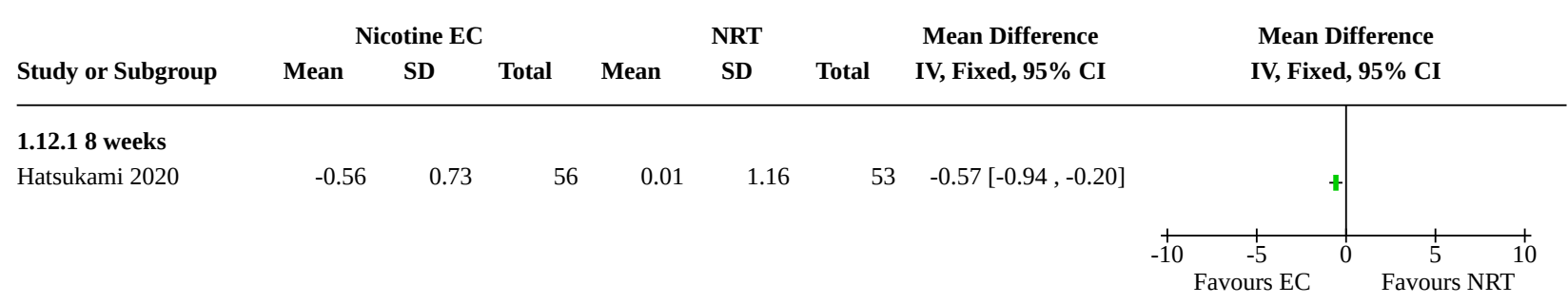

Analysis 1.13. Comparison 1: Nicotine EC versus NRT, Outcome 13: CEMA (pmol/mg creatinine)

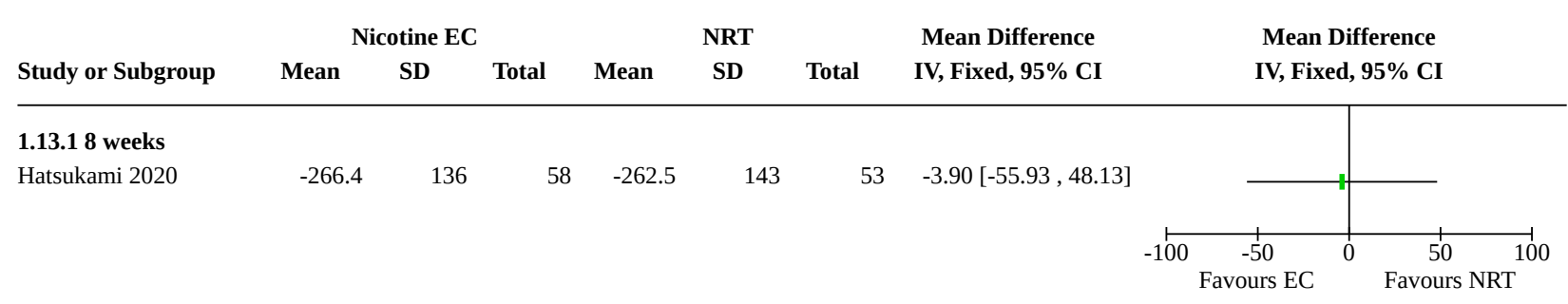

Analysis 1.14. Comparison 1: Nicotine EC versus NRT, Outcome 14: AAMA (pmol/mg creatinine)

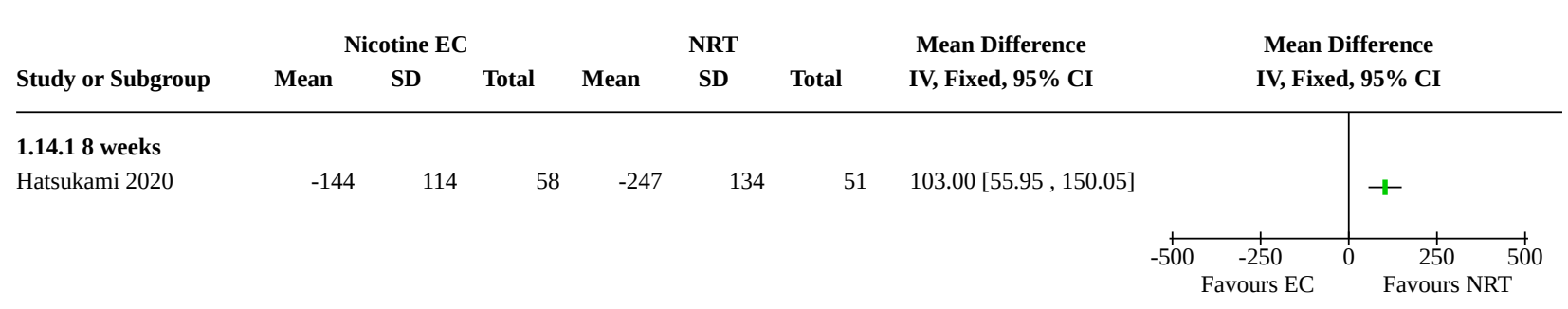


Analysis 1.15. Comparison 1: Nicotine EC versus NRT, Outcome 15: FEV1 (ml)

\begin{tabular}{lcccccccc} 
& \multicolumn{3}{c}{ Nicotine EC } & & NRT & & Mean Difference & Mean Difference \\
Study or Subgroup & Mean & SD & Total & Mean & SD & Total & IV, Fixed, 95\% CI & IV, Fixed, 95\% CI
\end{tabular}

1.15.1 8 weeks

$\begin{array}{llllllll}\text { Lee } 2018 & 292 & 503 & 18 & -300 & 549 & 8 & 592.00[146.22,1037.78\end{array}$

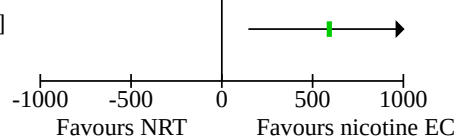

Analysis 1.16. Comparison 1: Nicotine EC versus NRT, Outcome 16: FEV1/FVC (\%)

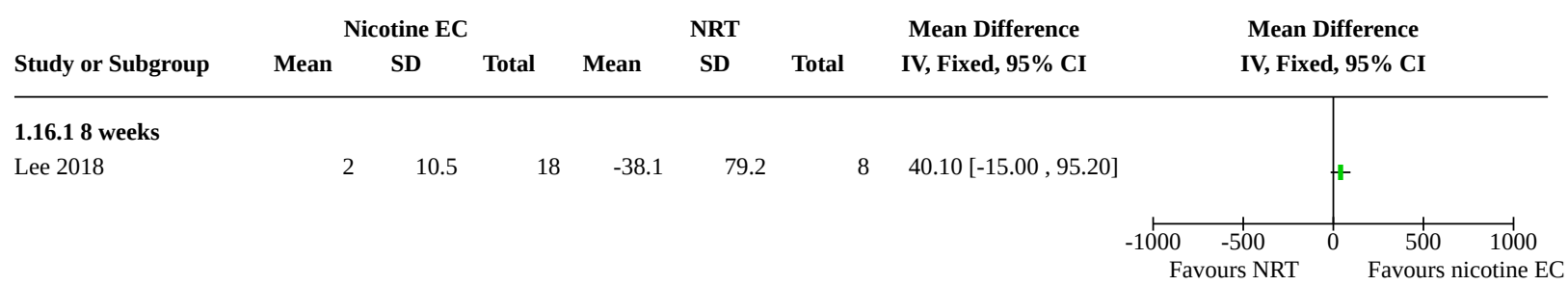

\section{Comparison 2. Nicotine EC versus varenicline}

\begin{tabular}{llllll}
\hline Outcome or subgroup title & No. of studies & $\begin{array}{l}\text { No. of partici- } \\
\text { pants }\end{array}$ & Statistical method & Effect size \\
\hline 2.1 Smoking cessation & 1 & & Risk Ratio (M-H, Fixed, 95\% Cl) & Totals not selected \\
\hline 2.2 Serious adverse events & 1 & 54 & Risk Ratio (M-H, Fixed, 95\% Cl) & Not estimable \\
\hline 2.2 .112 weeks & 1 & 54 & Risk Ratio (M-H, Fixed, 95\% Cl) & Not estimable \\
\hline
\end{tabular}

Analysis 2.1. Comparison 2: Nicotine EC versus varenicline, Outcome 1: Smoking cessation

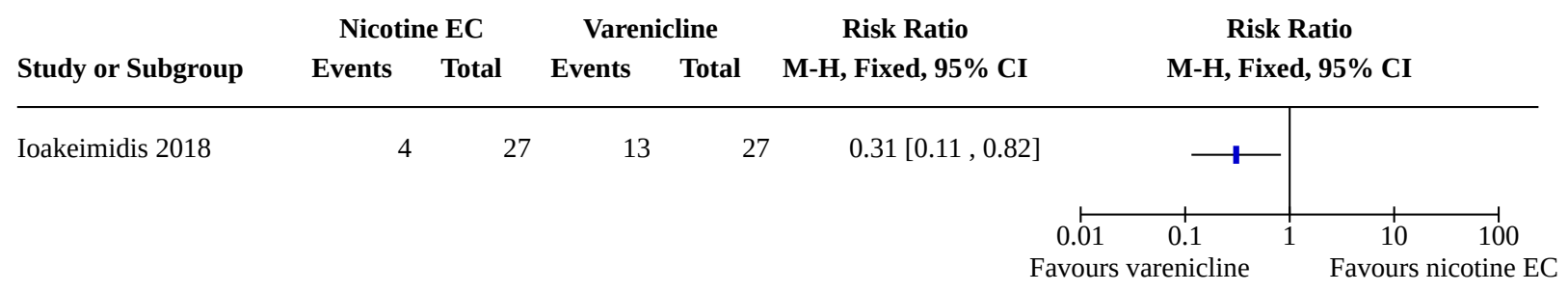




\section{Analysis 2.2. Comparison 2: Nicotine EC versus varenicline, Outcome 2: Serious adverse events}

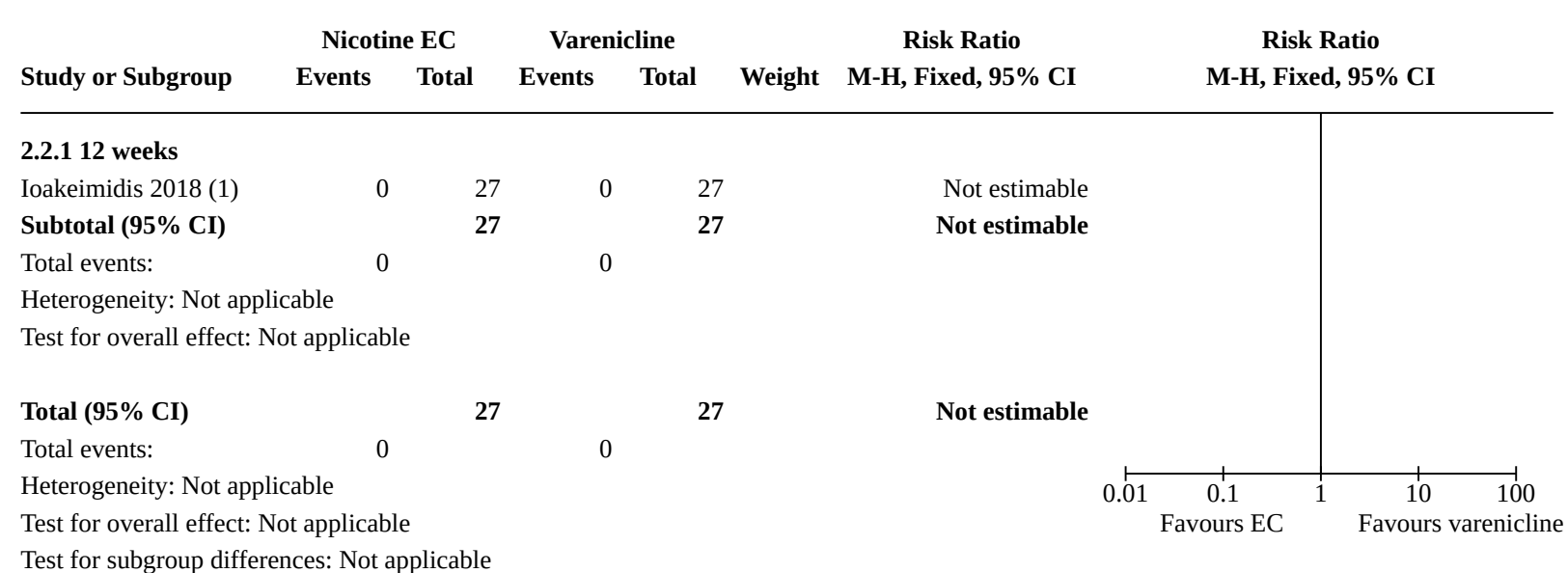

Footnotes

(1) $\mathrm{n}$ followed up not reported; $\mathrm{n}$ randomised used as denominators

Comparison 3. Nicotine EC versus non-nicotine EC

\begin{tabular}{|c|c|c|c|c|}
\hline $\begin{array}{l}\text { Outcome or sub- } \\
\text { group title }\end{array}$ & No. of studies & $\begin{array}{l}\text { No. of partici- } \\
\text { pants }\end{array}$ & Statistical method & Effect size \\
\hline $\begin{array}{l}3.1 \text { Smoking cessa- } \\
\text { tion }\end{array}$ & 4 & 1057 & Risk Ratio (M-H, Fixed, 95\% Cl) & $1.70[1.03,2.81]$ \\
\hline 3.2 Adverse events & 3 & 601 & Risk Ratio (M-H, Fixed, 95\% Cl) & $1.01[0.91,1.11]$ \\
\hline 3.2.11 week & 1 & 48 & Risk Ratio (M-H, Fixed, 95\% Cl) & $1.50[0.27,8.19]$ \\
\hline 3.2.2 6 months & 1 & 298 & Risk Ratio (M-H, Fixed, 95\% Cl) & $0.97[0.71,1.34]$ \\
\hline 3.2.3 12 weeks & 1 & 255 & Risk Ratio (M-H, Fixed, 95\% Cl) & $1.01[0.94,1.08]$ \\
\hline $\begin{array}{l}3.3 \text { Serious adverse } \\
\text { events }\end{array}$ & 4 & 494 & Risk Ratio (M-H, Fixed, 95\% Cl) & $0.60[0.15,2.44]$ \\
\hline 3.3.1 1 week & 1 & 48 & Risk Ratio (M-H, Fixed, 95\% Cl) & Not estimable \\
\hline 3.3.2 4 weeks & 1 & 74 & Risk Ratio (M-H, Fixed, 95\% Cl) & Not estimable \\
\hline 3.3.324 weeks & 1 & 255 & Risk Ratio (M-H, Fixed, 95\% Cl) & $0.60[0.15,2.44]$ \\
\hline 3.3.4 1 year & 1 & 117 & Risk Ratio (M-H, Fixed, 95\% Cl) & Not estimable \\
\hline $\begin{array}{l}\text { 3.4 Carbon monoxide } \\
(\mathrm{ppm})\end{array}$ & 2 & 171 & Mean Difference (IV, Fixed, 95\% CI) & $-2.44[-3.91,-0.97]$ \\
\hline 3.4.12 weeks & 1 & 25 & Mean Difference (IV, Fixed, 95\% CI) & $-0.40[-3.00,2.20]$ \\
\hline 3.4.2 12 weeks & 1 & 146 & Mean Difference (IV, Fixed, 95\% CI) & $-3.40[-5.18,-1.62]$ \\
\hline 3.5 Heart rate & 1 & & Mean Difference (IV, Fixed, 95\% CI) & Totals not selected \\
\hline
\end{tabular}




\begin{tabular}{|c|c|c|c|c|}
\hline $\begin{array}{l}\text { Outcome or sub- } \\
\text { group title }\end{array}$ & No. of studies & $\begin{array}{l}\text { No. of partici- } \\
\text { pants }\end{array}$ & Statistical method & Effect size \\
\hline 3.5.1 12 weeks & 1 & & Mean Difference (IV, Fixed, 95\% CI) & Totals not selected \\
\hline $\begin{array}{l}3.6 \text { Systolic blood } \\
\text { pressure }\end{array}$ & 1 & & Mean Difference (IV, Fixed, 95\% CI) & Totals not selected \\
\hline 3.6.1 12 weeks & 1 & & Mean Difference (IV, Fixed, 95\% CI) & Totals not selected \\
\hline 3.7 FeNO (ppb) & 1 & & Mean Difference (IV, Fixed, 95\% CI) & Totals not selected \\
\hline 3.7.1 12 weeks & 1 & & Mean Difference (IV, Fixed, 95\% CI) & Totals not selected \\
\hline 3.8 FEV1 (l) & 1 & & Std. Mean Difference (IV, Fixed, 95\% Cl) & Totals not selected \\
\hline 3.8.1 12 weeks & 1 & & Std. Mean Difference (IV, Fixed, 95\% CI) & Totals not selected \\
\hline 3.9 FVC (l) & 1 & & Mean Difference (IV, Fixed, 95\% Cl) & Totals not selected \\
\hline 3.9.1 12 weeks & 1 & & Mean Difference (IV, Fixed, 95\% CI) & Totals not selected \\
\hline 3.10 FEV1/FVC & 1 & & Mean Difference (IV, Fixed, 95\% CI) & Totals not selected \\
\hline 3.10 .112 weeks & 1 & & Mean Difference (IV, Fixed, 95\% CI) & Totals not selected \\
\hline
\end{tabular}

\section{Analysis 3.1. Comparison 3: Nicotine EC versus non-nicotine EC, Outcome 1: Smoking cessation}

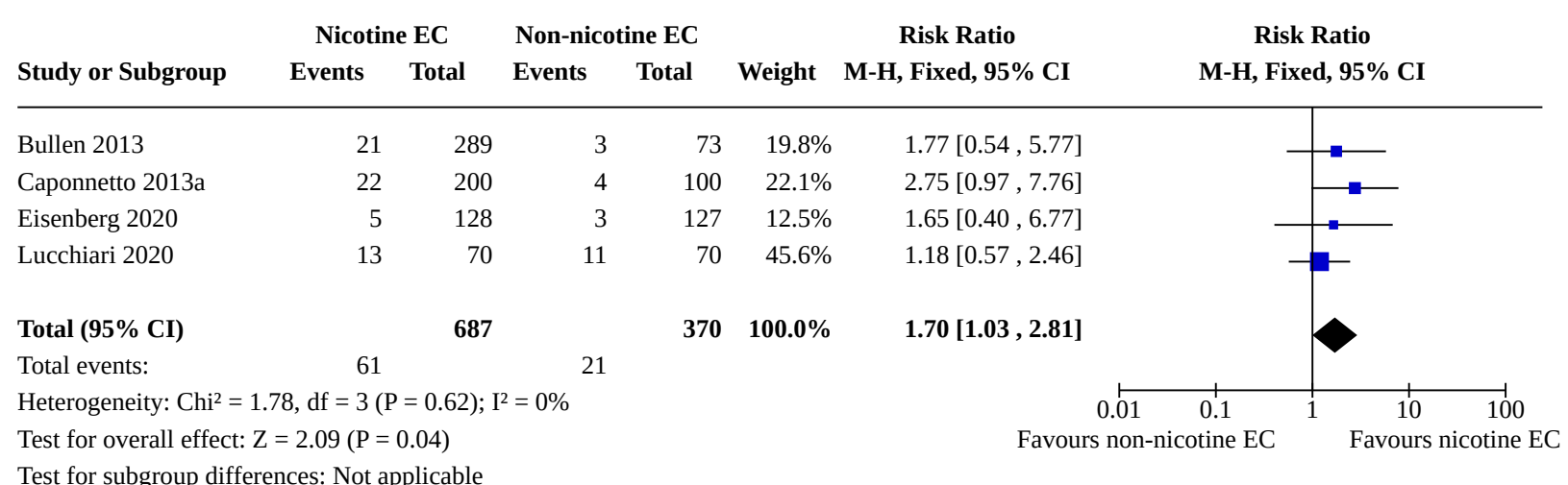


Analysis 3.2. Comparison 3: Nicotine EC versus non-nicotine EC, Outcome 2: Adverse events

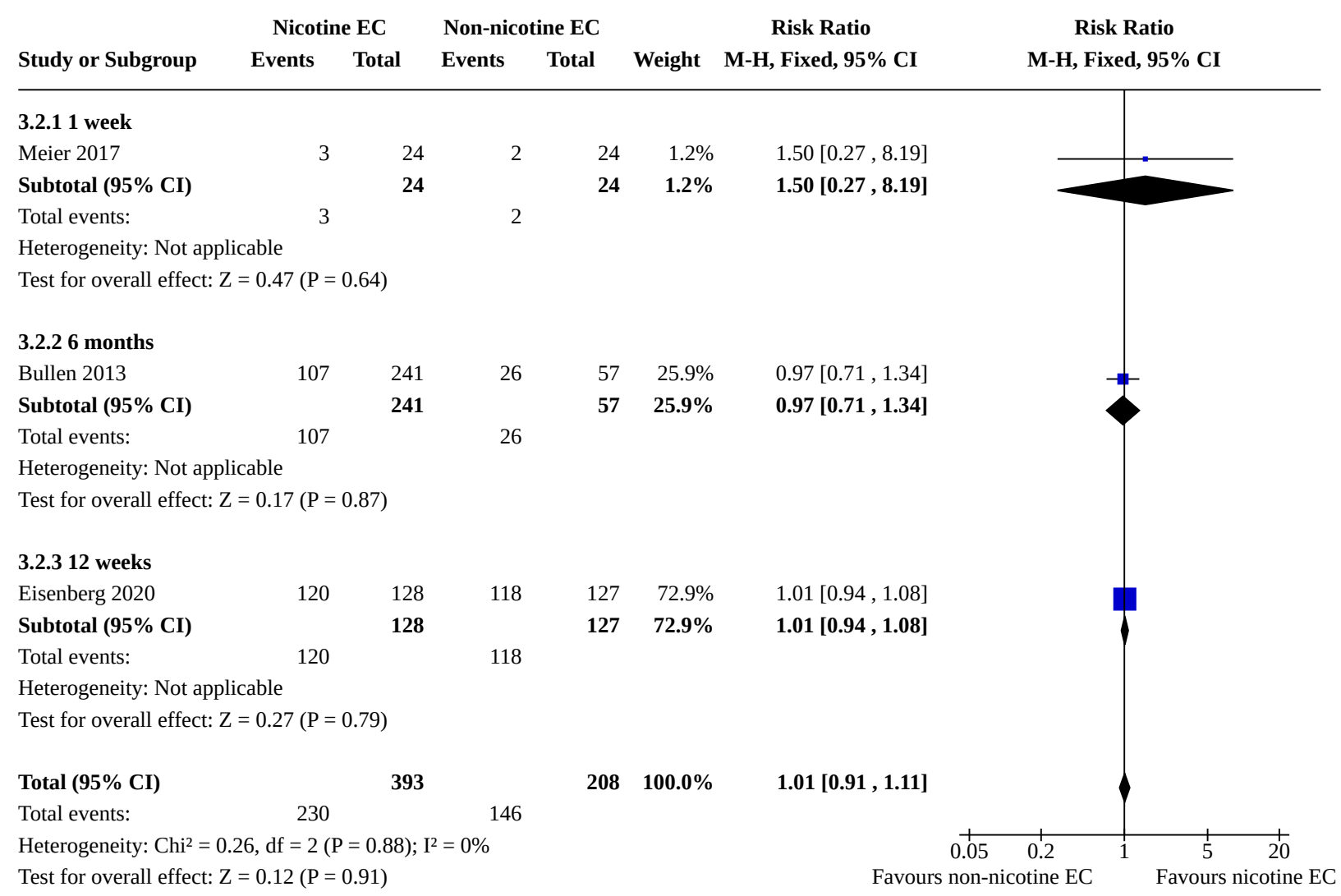


Analysis 3.3. Comparison 3: Nicotine EC versus non-nicotine EC, Outcome 3: Serious adverse events

\begin{tabular}{|c|c|c|c|c|c|c|c|}
\hline & \multicolumn{2}{|c|}{ Nicotine EC } & Non-ni & ne EC & \multicolumn{2}{|r|}{ Risk Ratio } & Risk Ratio \\
\hline Study or Subgroup & Events & Total & Events & Total & Weight & M-H, Fixed, 95\% CI & M-H, Fixed, 95\% CI \\
\hline
\end{tabular}

\begin{tabular}{lccccr}
\hline 3.3.1 1 week & & & & & \\
Meier 2017 & 0 & 24 & 0 & 24 & Not estimable \\
Subtotal (95\% CI) & & $\mathbf{2 4}$ & & $\mathbf{2 4}$ & Not estimable \\
Total events: & 0 & & 0 & &
\end{tabular}

Heterogeneity: Not applicable

Test for overall effect: Not applicable

3.3.2 4 weeks

George 2019

Subtotal (95\% CI)

Total events:

Heterogeneity: Not applicable

Test for overall effect: Not applicable

\subsubsection{4 weeks}

Eisenberg 2020

Subtotal (95\% CI)

Total events:

Heterogeneity: Not applicable

Test for overall effect: $\mathrm{Z}=0.72(\mathrm{P}=0.47)$

\subsubsection{1 year}

Caponnetto 2013a

Subtotal (95\% CI)

0

72

Total events:

0

Heterogeneity: Not applicable

Test for overall effect: Not applicable

\section{Total $(95 \%$ CI)}

261

Total events:

3

Heterogeneity: Not applicable

Test for overall effect: $\mathrm{Z}=0.72(\mathrm{P}=0.47)$

Test for subgroup differences: Not applicable
Not estimable

Not estimable

\section{7}

$127 \quad \mathbf{1 0 0 . 0} \%$

$0.60[0.15,2.44]$

$0.60[0.15,2.44]$

Not estimable Not estimable
45

45

$233 \quad 100.0 \%$ 5
$0.60[0.15,2.44]$ 
Analysis 3.4. Comparison 3: Nicotine EC versus non-nicotine EC, Outcome 4: Carbon monoxide (ppm)

\begin{tabular}{|c|c|c|c|c|c|c|c|c|c|}
\hline \multirow{2}{*}{ Study or Subgroup } & \multicolumn{3}{|c|}{ Nicotine EC } & \multicolumn{3}{|c|}{ Non-nicotine EC } & \multirow{2}{*}{\multicolumn{2}{|c|}{$\begin{array}{c}\text { Mean Difference } \\
\text { IV, Fixed, 95\% CI }\end{array}$}} & Mean Difference \\
\hline & Mean & SD & Total & Mean & SD & Total & & & IV, Fixed, 95\% CI \\
\hline
\end{tabular}

\begin{tabular}{lllllllll}
\hline 3.4.1 2 weeks & & & & & & & & \\
Felicione 2019 & -1.9 & 3.4 & 14 & -1.5 & 3.2 & 11 & $32.0 \%$ & $-0.40[-3.00,2.20]$ \\
Subtotal (95\% CI) & & & $\mathbf{1 4}$ & & & $\mathbf{1 1}$ & $\mathbf{3 2 . 0} \%$ & $\mathbf{- 0 . 4 0}[-\mathbf{3 . 0 0}, \mathbf{2 . 2 0}]$
\end{tabular}

Heterogeneity: Not applicable

Test for overall effect: $\mathrm{Z}=0.30(\mathrm{P}=0.76)$

3.4.2 12 weeks

$\begin{array}{lllllllll}\text { Caponnetto 2013a (1) } & -6 & 6.4 & 76 & -2.6 & 4.5 & 70 & 68.0 \% & -3.40[-5.18,-1.62]\end{array}$

$\begin{array}{lllll}\text { Subtotal }(95 \% \mathrm{CI}) & 76 & 70 & \mathbf{6 8 . 0} \% & -\mathbf{3 . 4 0}[-5.18,-1.62]\end{array}$

Heterogeneity: Not applicable

Test for overall effect: $\mathrm{Z}=3.74(\mathrm{P}=0.0002)$

Total (95\% CI)

90

$81 \quad 100.0 \% \quad-2.44[-3.91,-0.97]$

Heterogeneity: Chi $^{2}=3.48, \mathrm{df}=1(\mathrm{P}=0.06) ; \mathrm{I}^{2}=71 \%$

Test for overall effect: $\mathrm{Z}=3.25(\mathrm{P}=0.001)$

Test for subgroup differences: $\mathrm{Chi}^{2}=3.48, \mathrm{df}=1(\mathrm{P}=0.06), \mathrm{I}^{2}=71.3 \%$

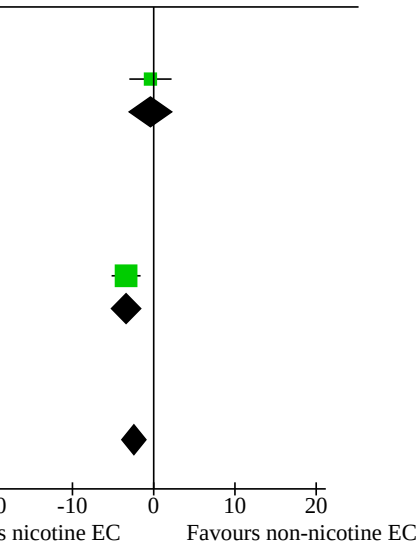

Footnotes

(1) Data is $2.4 \%$ nicotine compared to no-nicotine; $1.8 \%$ nicotine arm reported elsewhere

\section{Analysis 3.5. Comparison 3: Nicotine EC versus non-nicotine EC, Outcome 5: Heart rate}

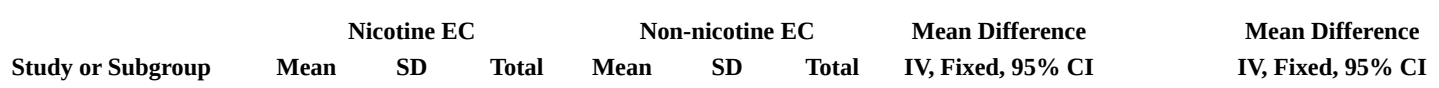

$\begin{array}{lllllll}\text { Study or Subgroup } & \text { Mean } & \text { SD } & \text { Total } & \text { Mean } & \text { SD } & \text { Total IV, Fixed, 95\% CI }\end{array}$

3.5.1 12 weeks

Caponnetto 2013a (1) $\quad-1.7 \quad 3.4 \quad 73 \quad 1.1 \quad 33 \quad 68 \quad-2.80[-3.86,-1.74]$

Footnotes

(1) Data is $2.4 \%$ nicotine compared to no-nicotine; $1.8 \%$ nicotine arm reported elsewhere

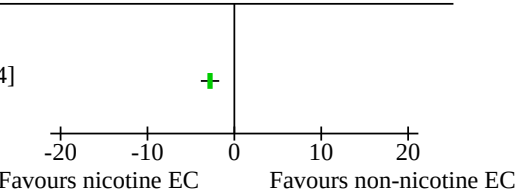

Analysis 3.6. Comparison 3: Nicotine EC versus non-nicotine EC, Outcome 6: Systolic blood pressure

\begin{tabular}{lcccccccc} 
& \multicolumn{2}{c}{ Nicotine EC } & \multicolumn{2}{c}{ Non-nicotine EC } & Mean Difference & Mean Difference \\
Study or Subgroup & Mean & SD & Total & Mean & SD & Total & IV, Fixed, 95\% CI & IV, Fixed, 95\% CI
\end{tabular}

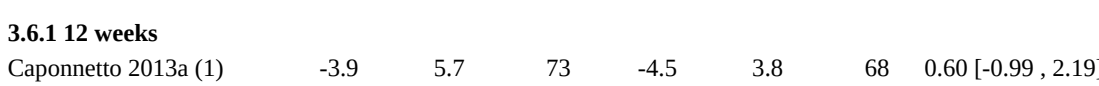

Footnotes

(1) Data is $2.4 \%$ nicotine compared to no-nicotine; $1.8 \%$ nicotine arm reported elsewhere

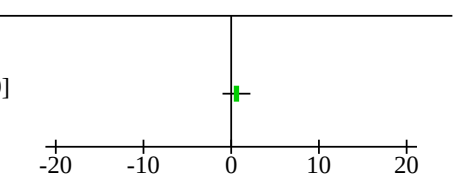

Favours nicotine EC Favours non-nicotine EC 
Analysis 3.7. Comparison 3: Nicotine EC versus non-nicotine EC, Outcome 7: FeNO (ppb)

\begin{tabular}{lcccccccc} 
& \multicolumn{3}{c}{ Nicotine EC } & \multicolumn{2}{c}{ Non-nicotine EC } & Mean Difference & Mean Difference \\
Study or Subgroup & Mean & SD & Total & Mean & SD & Total & IV, Fixed, 95\% CI & IV, Fixed, 95\% CI
\end{tabular}

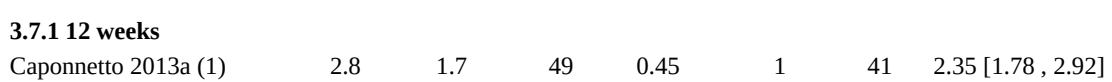

Footnotes

(1) Data is $2.4 \%$ nicotine compared to no-nicotine; $1.8 \%$ nicotine arm reported elsewhere

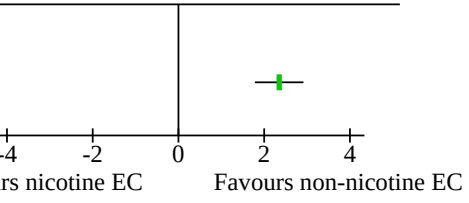

\section{Analysis 3.8. Comparison 3: Nicotine EC versus non-nicotine EC, Outcome 8: FEV1 (l)}

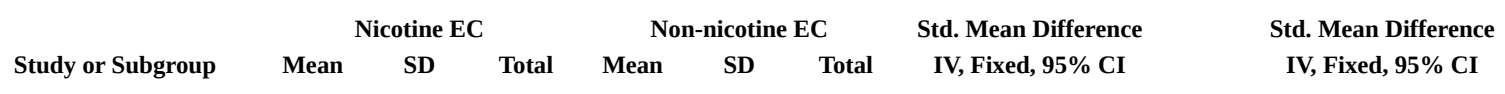

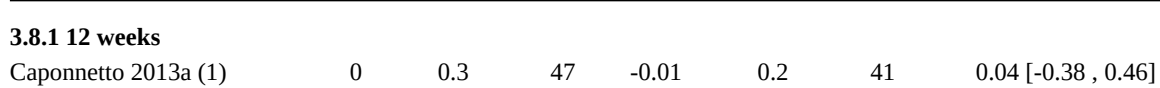

Footnotes

(1) Data is $2.4 \%$ nicotine compared to no-nicotine; $1.8 \%$ nicotine arm reported elsewhere

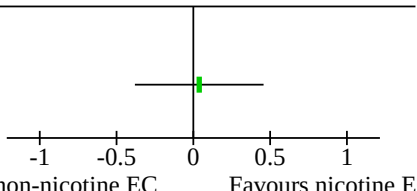

Analysis 3.9. Comparison 3: Nicotine EC versus non-nicotine EC, Outcome 9: FVC (I)

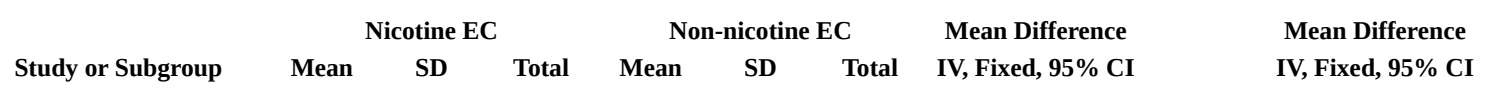

\begin{tabular}{llllllll}
\hline 3.9.1 12 weeks & & & & & & & \\
Caponnetto 2013a (1) & -0.02 & 0.3 & 47 & -0.07 & 0.3 & 41 & $0.05[-0.08,0.18]$
\end{tabular}

3.9.1 12 weeks

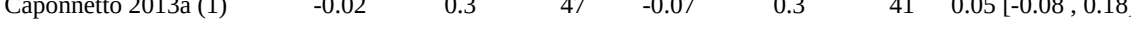

Footnotes

non-nicotine EC

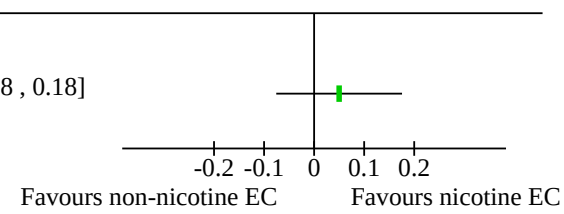

(1) Data is $2.4 \%$ nicotine compared to no-nicotine; $1.8 \%$ nicotine arm reported elsewhere

Analysis 3.10. Comparison 3: Nicotine EC versus non-nicotine EC, Outcome 10: FEV1/FVC

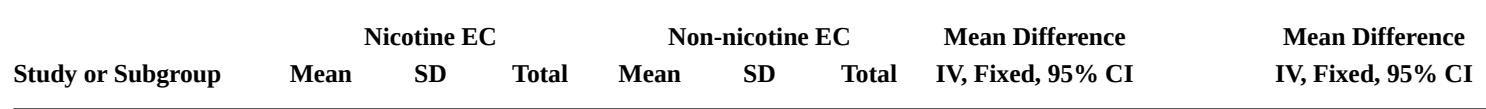

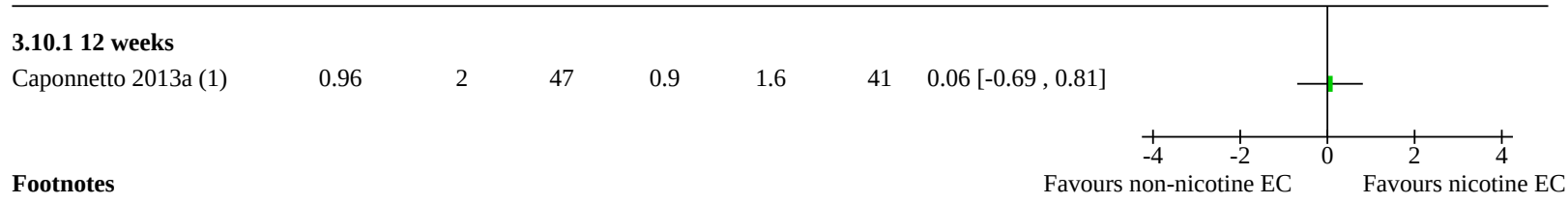

(1) Data is $2.4 \%$ nicotine compared to no-nicotine; $1.8 \%$ nicotine arm reported elsewhere

Comparison 4. Nicotine EC versus behavioural support only/no support

\begin{tabular}{lllll}
\hline $\begin{array}{l}\text { Outcome or sub- } \\
\text { group title }\end{array}$ & No. of studies & $\begin{array}{l}\text { No. of partici- } \\
\text { pants }\end{array}$ & Statistical method & Effect size \\
\hline 4.1 Smoking cessation & 5 & 2561 & Risk Ratio (M-H, Fixed, 95\% Cl) & $2.70[1.39,5.26]$ \\
\hline
\end{tabular}




\begin{tabular}{|c|c|c|c|c|}
\hline $\begin{array}{l}\text { Outcome or sub- } \\
\text { group title }\end{array}$ & No. of studies & $\begin{array}{l}\text { No. of partici- } \\
\text { pants }\end{array}$ & Statistical method & Effect size \\
\hline 4.2 Adverse events & 4 & 765 & Risk Ratio (M-H, Fixed, 95\% Cl) & $1.22[1.12,1.32]$ \\
\hline 4.2.1 12 weeks & 2 & 657 & Risk Ratio (M-H, Fixed, 95\% CI) & $1.20[1.11,1.30]$ \\
\hline 4.2.2 16 weeks & 1 & 50 & Risk Ratio (M-H, Fixed, 95\% Cl) & $1.18[0.67,2.07]$ \\
\hline 4.2.3 6 months & 1 & 58 & Risk Ratio (M-H, Fixed, 95\% Cl) & $11.00[0.64,190.26]$ \\
\hline $\begin{array}{l}4.3 \text { Serious adverse } \\
\text { events }\end{array}$ & 6 & 1011 & Risk Ratio (M-H, Fixed, 95\% Cl) & $1.17[0.33,4.09]$ \\
\hline 4.3.1 4 to 6 weeks & 2 & 246 & Risk Ratio (M-H, Fixed, 95\% Cl) & Not estimable \\
\hline 4.3.2 12 weeks & 1 & 408 & Risk Ratio (M-H, Fixed, 95\% CI) & $3.69[0.21,66.17]$ \\
\hline 4.3.3 16 weeks & 1 & 50 & Risk Ratio (M-H, Fixed, 95\% Cl) & Not estimable \\
\hline 4.3.4 6 months & 2 & 307 & Risk Ratio (M-H, Fixed, 95\% Cl) & $0.71[0.16,3.10]$ \\
\hline $\begin{array}{l}\text { 4.4 Carbon monoxide } \\
\text { (ppm) }\end{array}$ & 8 & & Mean Difference (IV, Fixed, 95\% CI) & Totals not selected \\
\hline 4.4.13 to 4 weeks & 3 & & Mean Difference (IV, Fixed, 95\% CI) & Totals not selected \\
\hline 4.4.2 6 weeks & 1 & & Mean Difference (IV, Fixed, 95\% CI) & Totals not selected \\
\hline 4.4.3 8 weeks & 2 & & Mean Difference (IV, Fixed, 95\% CI) & Totals not selected \\
\hline 4.4.4 4 months & 1 & & Mean Difference (IV, Fixed, 95\% CI) & Totals not selected \\
\hline 4.4.5 6 months & 1 & & Mean Difference (IV, Fixed, 95\% CI) & Totals not selected \\
\hline 4.5 Heart rate (bpm) & 1 & & Mean Difference (IV, Fixed, 95\% CI) & Totals not selected \\
\hline 4.5.1 8 weeks & 1 & & Mean Difference (IV, Fixed, 95\% CI) & Totals not selected \\
\hline $\begin{array}{l}4.6 \text { Systolic blood } \\
\text { pressure }\end{array}$ & 3 & & Mean Difference (IV, Fixed, 95\% CI) & Totals not selected \\
\hline 4.6.1 6 weeks & 1 & & Mean Difference (IV, Fixed, 95\% CI) & Totals not selected \\
\hline 4.6.2 8 weeks & 1 & & Mean Difference (IV, Fixed, 95\% CI) & Totals not selected \\
\hline 4.6.3 4 months & 1 & & Mean Difference (IV, Fixed, 95\% CI) & Totals not selected \\
\hline $\begin{array}{l}4.7 \text { Blood oxygen satu- } \\
\text { ration }\end{array}$ & 1 & & Mean Difference (IV, Fixed, 95\% CI) & Totals not selected \\
\hline 4.7.18 weeks & 1 & & Mean Difference (IV, Fixed, 95\% CI) & Totals not selected \\
\hline 4.8 3-HPMA (SMD) & 2 & & Std. Mean Difference (IV, Fixed, 95\% CI) & Totals not selected \\
\hline 4.8.1 8 weeks & 1 & & Std. Mean Difference (IV, Fixed, 95\% CI) & Totals not selected \\
\hline
\end{tabular}




\begin{tabular}{|c|c|c|c|c|}
\hline $\begin{array}{l}\text { Outcome or sub- } \\
\text { group title }\end{array}$ & No. of studies & $\begin{array}{l}\text { No. of partici- } \\
\text { pants }\end{array}$ & Statistical method & Effect size \\
\hline 4.8.2 12 weeks & 1 & & Std. Mean Difference (IV, Fixed, 95\% CI) & Totals not selected \\
\hline 4.9 NNAL (SMD) & 4 & & Std. Mean Difference (IV, Fixed, 95\% CI) & Totals not selected \\
\hline 4.9.13 weeks & 1 & & Std. Mean Difference (IV, Fixed, 95\% CI) & Totals not selected \\
\hline 4.9.2 8 weeks & 1 & & Std. Mean Difference (IV, Fixed, 95\% CI) & Totals not selected \\
\hline 4.9.3 12 weeks & 1 & & Std. Mean Difference (IV, Fixed, 95\% CI) & Totals not selected \\
\hline 4.9.4 6 weeks & 1 & & Std. Mean Difference (IV, Fixed, 95\% CI) & Totals not selected \\
\hline $\begin{array}{l}4.102-\mathrm{HPMA} \text { (pmol/ } \\
\mathrm{mg} \text { creatinine) }\end{array}$ & 1 & & Mean Difference (IV, Fixed, 95\% CI) & Totals not selected \\
\hline 4.10.18 weeks & 1 & & Mean Difference (IV, Fixed, 95\% CI) & Totals not selected \\
\hline $\begin{array}{l}4.11 \text { HMPMA (pmol/ } \\
\text { mg creatinine) }\end{array}$ & 1 & & Mean Difference (IV, Fixed, 95\% CI) & Totals not selected \\
\hline 4.11.1 8 weeks & 1 & & Mean Difference (IV, Fixed, 95\% CI) & Totals not selected \\
\hline $\begin{array}{l}4.12 \mathrm{PheT}(\mathrm{pmol} / \mathrm{mg} \\
\text { creatinine) }\end{array}$ & 1 & & Mean Difference (IV, Fixed, 95\% CI) & Totals not selected \\
\hline 4.12.1 8 weeks & 1 & & Mean Difference (IV, Fixed, 95\% CI) & Totals not selected \\
\hline $\begin{array}{l}4.13 \text { CEMA (pmol/mg } \\
\text { creatinine) }\end{array}$ & 1 & & Mean Difference (IV, Fixed, 95\% CI) & Totals not selected \\
\hline 4.13.1 8 weeks & 1 & & Mean Difference (IV, Fixed, 95\% CI) & Totals not selected \\
\hline $\begin{array}{l}\text { 4.14 AAMA (pmol/mg } \\
\text { creatinine) }\end{array}$ & 1 & & Mean Difference (IV, Fixed, 95\% CI) & Totals not selected \\
\hline 4.14.1 8 weeks & 1 & & Mean Difference (IV, Fixed, 95\% CI) & Totals not selected \\
\hline $\begin{array}{l}\text { 4.15 S-PMA } \\
\text { (nanograms) }\end{array}$ & 1 & & Mean Difference (IV, Fixed, 95\% CI) & Totals not selected \\
\hline 4.15.1 12 weeks & 1 & & Mean Difference (IV, Fixed, 95\% CI) & Totals not selected \\
\hline 4.16 FVC (litres) & 1 & & Mean Difference (IV, Fixed, 95\% CI) & Totals not selected \\
\hline 4.16.1 12 weeks & 1 & & Mean Difference (IV, Fixed, 95\% CI) & Totals not selected \\
\hline 4.17 FEV1 (litres) & 1 & & Mean Difference (IV, Fixed, 95\% CI) & Totals not selected \\
\hline 4.17.1 12 weeks & 1 & & Mean Difference (IV, Fixed, 95\% CI) & Totals not selected \\
\hline $\begin{array}{l}\text { 4.18 FEF 25-75 (litres/ } \\
\text { second)) }\end{array}$ & 2 & 555 & Mean Difference (IV, Fixed, 95\% CI) & $-0.06[-0.18,0.06]$ \\
\hline 4.18.1 6 weeks & 1 & 168 & Mean Difference (IV, Fixed, 95\% CI) & $-0.14[-0.28,0.00]$ \\
\hline
\end{tabular}




\begin{tabular}{lllll}
\hline $\begin{array}{l}\text { Outcome or sub- } \\
\text { group title }\end{array}$ & No. of studies & $\begin{array}{l}\text { No. of partici- } \\
\text { pants }\end{array}$ & Statistical method & Effect size \\
\hline 4.18 .212 weeks & 1 & 387 & Mean Difference (IV, Fixed, 95\% Cl) & $0.10[-0.10,0.30]$ \\
\hline $\begin{array}{l}4.19 \text { PEF 25-75 (litres/ } \\
\text { minute) }\end{array}$ & 1 & Mean Difference (IV, Fixed, 95\% CI) & Totals not selected \\
\hline 4.19 .112 weeks & 1 & Mean Difference (IV, Fixed, 95\% Cl) & Totals not selected \\
\hline
\end{tabular}

\section{Analysis 4.1. Comparison 4: Nicotine EC versus behavioural support only/no support, Outcome 1: Smoking cessation}

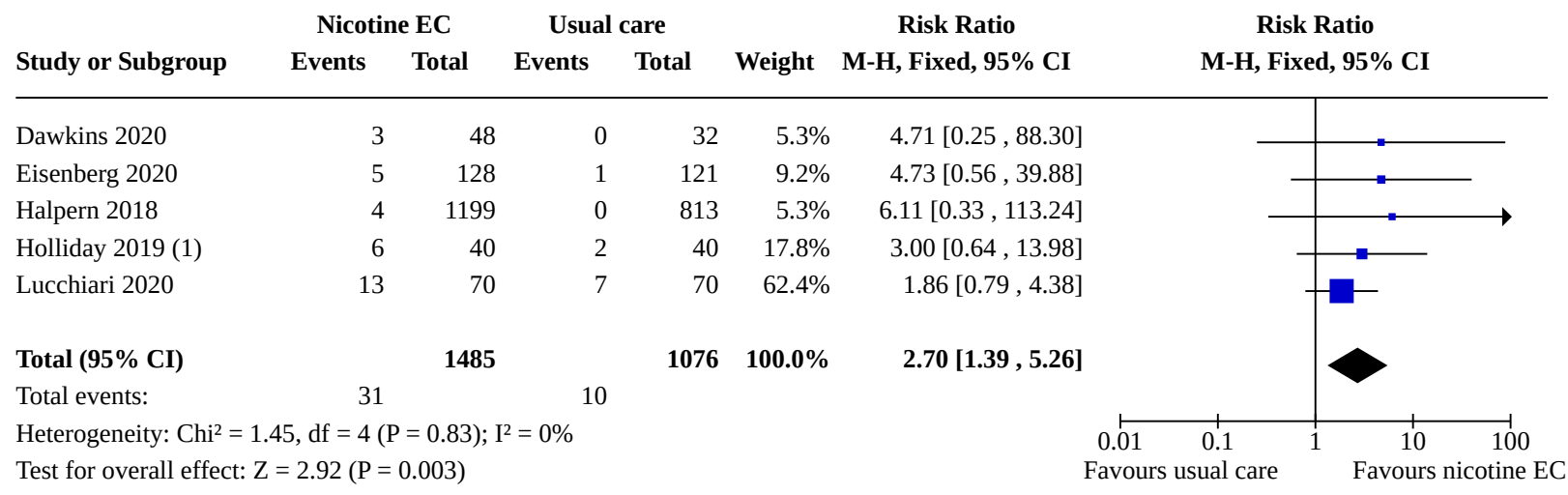

Test for subgroup differences: Not applicable

Footnotes

(1) Although participants were given a choice of nicotine concentration including $0 \mathrm{mg}$, none of the participants chose the non-nicotine e-liquid 
Analysis 4.2. Comparison 4: Nicotine EC versus behavioural support only/no support, Outcome 2: Adverse events

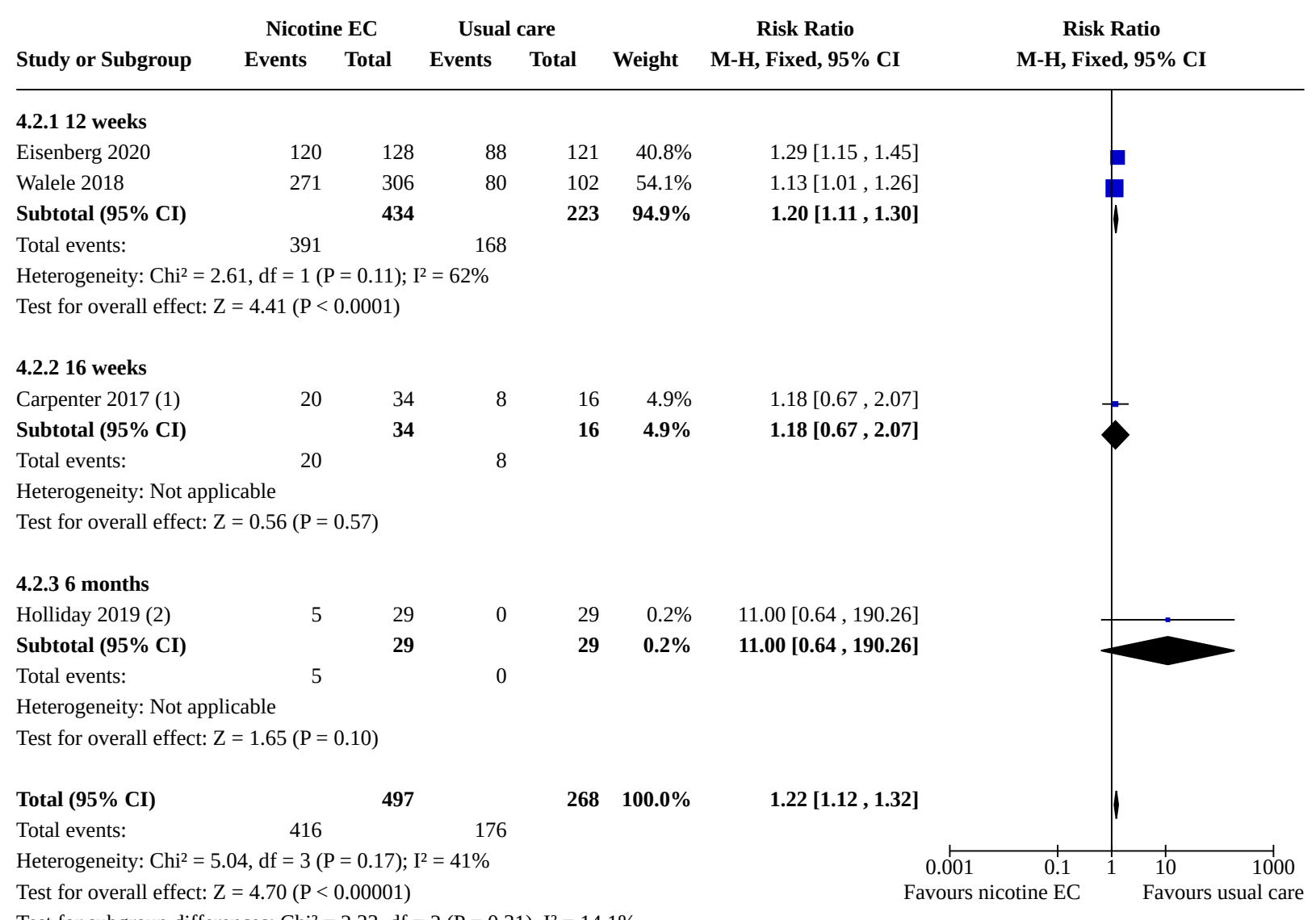

(1) 24mg EC arm included here; 16mg data reported elsewhere

(2) Participants offered choice of nicotine or no-nicotine EC; all chose nicotine-containing EC 


\section{Analysis 4.3. Comparison 4: Nicotine EC versus behavioural} support only/no support, Outcome 3: Serious adverse events

\begin{tabular}{lccccccc} 
& \multicolumn{2}{c}{ Nicotine EC } & \multicolumn{2}{c}{ Usual care } & \multicolumn{2}{c}{ Risk Ratio } & \multicolumn{2}{c}{ Risk Ratio } \\
Study or Subgroup & Events & Total & Events & Total & Weight & M-H, Fixed, 95\% CI & M-H, Fixed, 95\% CI
\end{tabular}

\begin{tabular}{lcrccr}
\hline 4.3.1 4 to 6 weeks & & & & & \\
George 2019 & 0 & 37 & 0 & 40 & Not estimable \\
Pulvers 2020 & 0 & 115 & 0 & 54 & Not estimable \\
Subtotal (95\% CI) & & $\mathbf{1 5 2}$ & & $\mathbf{9 4}$ & Not estimable \\
Total events: & 0 & & 0 & &
\end{tabular}

Heterogeneity: Not applicable

Test for overall effect: Not applicable

\subsubsection{2 weeks}

$\begin{array}{lcccccc}\text { Walele } 2018 & 5 & 306 & 0 & 102 & 15.4 \% & 3.69[0.21,66.17] \\ \text { Subtotal (95\% CI) } & & \mathbf{3 0 6} & & \mathbf{1 0 2} & \mathbf{1 5 . 4 \%} & \mathbf{3 . 6 9}[\mathbf{0 . 2 1 , \mathbf { 6 6 . 1 7 } ]} \\ \text { Total events: } & 5 & & 0 & & & \end{array}$

Heterogeneity: Not applicable

Test for overall effect: $\mathrm{Z}=0.89(\mathrm{P}=0.38)$

4.3.3 16 weeks

Carpenter 2017 (1)

Subtotal (95\% CI)

$0 \quad 34$

\section{0}

16

Not estimable

Total events:

$0 \quad 0$

Not estimable

Heterogeneity: Not applicable

Test for overall effect: Not applicable

\subsubsection{6 months}

Eisenberg 2020

Holliday 2019 (2)

Subtotal (95\% CI)

Total events:

Heterogeneity: Not applicable

Test for overall effect: $\mathrm{Z}=0.46(\mathrm{P}=0.65)$

Total (95\% CI)

649

Total events:

8

128

4

121

$84.6 \%$

$0.71[0.16,3.10]$

Not estimable

$150 \quad 84.6 \% \quad 0.71[0.16,3.10]$

$362 \quad 100.0 \%$

$1.17[0.33,4.09]$

Heterogeneity: $\mathrm{Chi}^{2}=1.05, \mathrm{df}=1(\mathrm{P}=0.31) ; \mathrm{I}^{2}=5 \%$

Test for overall effect: $\mathrm{Z}=0.24(\mathrm{P}=0.81)$

Test for subgroup differences: $\mathrm{Chi}^{2}=0.99$, $\mathrm{df}=1(\mathrm{P}=0.32), \mathrm{I}^{2}=0 \%$

Footnotes

(1) Data from 24mg arm (0 events in $16 \mathrm{mg}$ arm as well)

(2) Participants offered choice of nicotine or no-nicotine EC; all chose nicotine-containing EC 


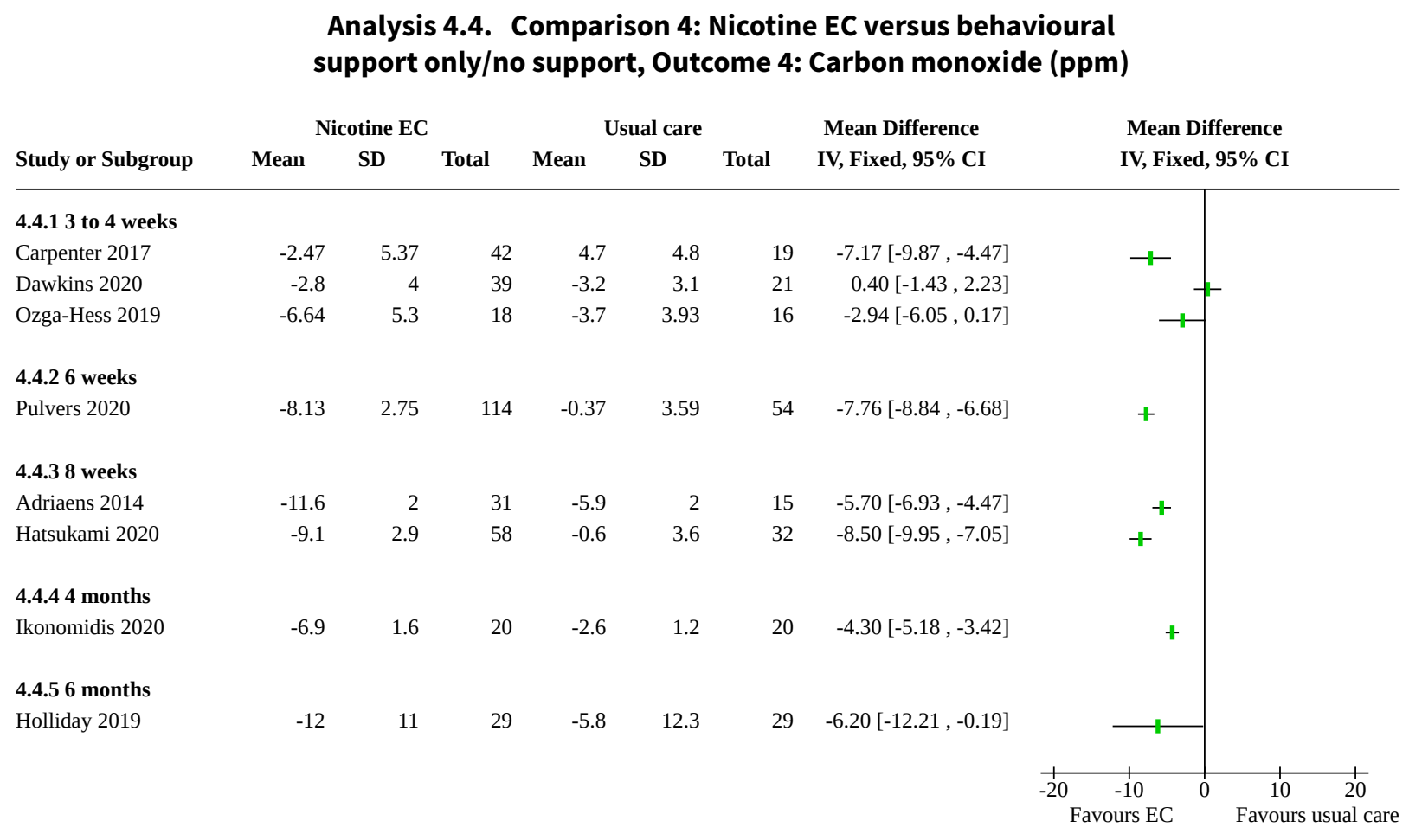

Analysis 4.5. Comparison 4: Nicotine EC versus behavioural support only/no support, Outcome 5: Heart rate (bpm)

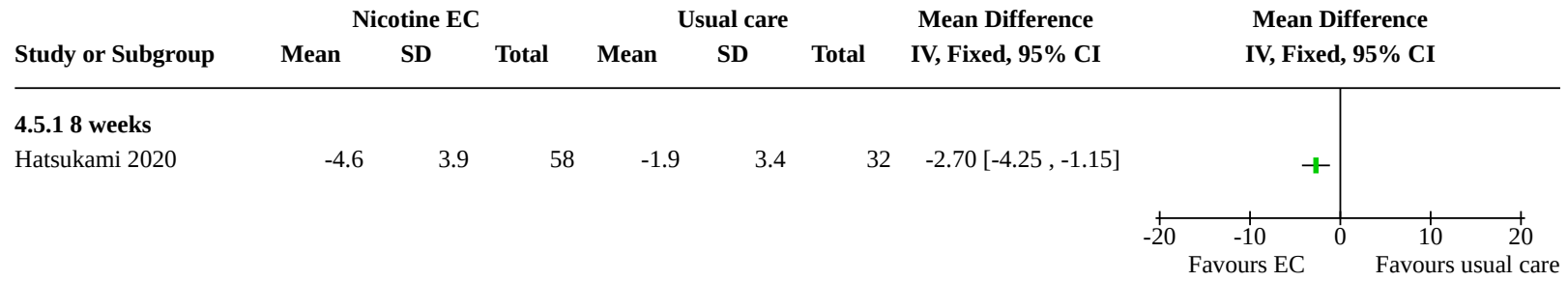

Analysis 4.6. Comparison 4: Nicotine EC versus behavioural support only/no support, Outcome 6: Systolic blood pressure

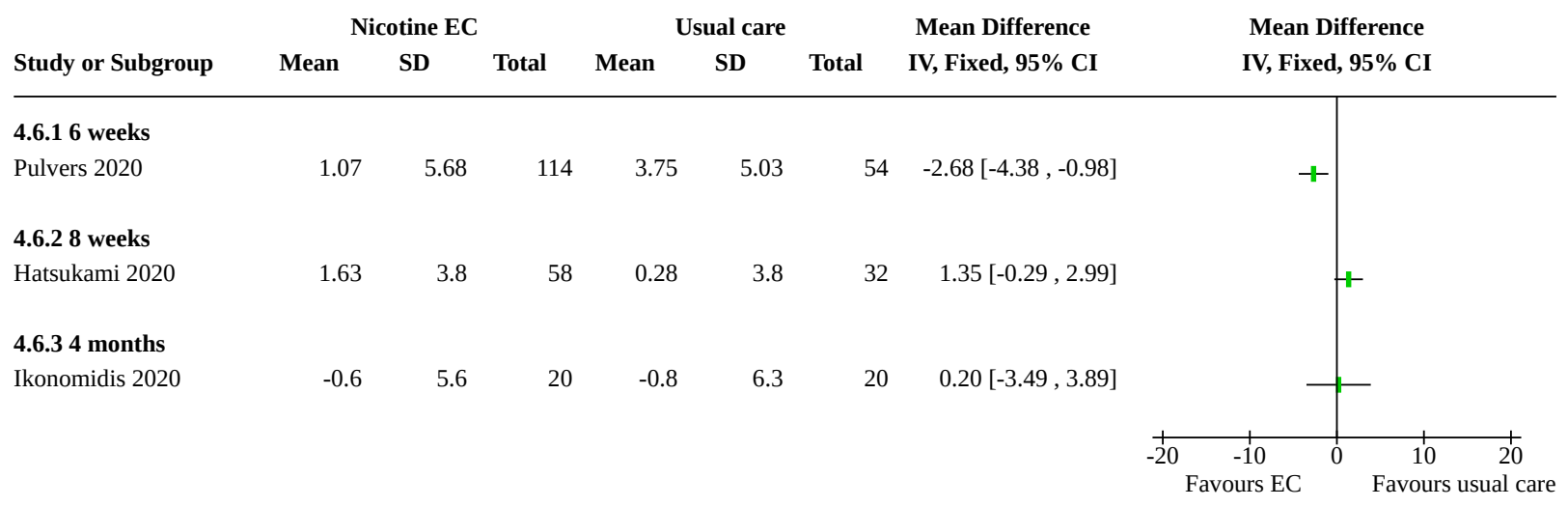


Analysis 4.7. Comparison 4: Nicotine EC versus behavioural support only/no support, Outcome 7: Blood oxygen saturation

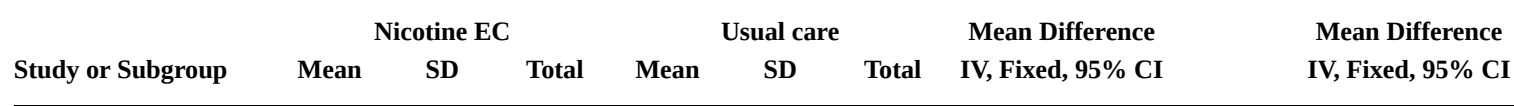

4.7.1 8 weeks

Hatsukami 2020

$\begin{array}{lllllll}0.2 & 0.5 & 57 & -0.3 & 0.4 & 32 & 0.50[0.31,0.69\end{array}$

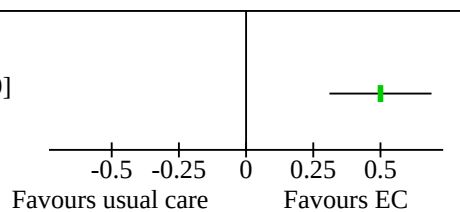

Analysis 4.8. Comparison 4: Nicotine EC versus behavioural support only/no support, Outcome 8: 3-HPMA (SMD)

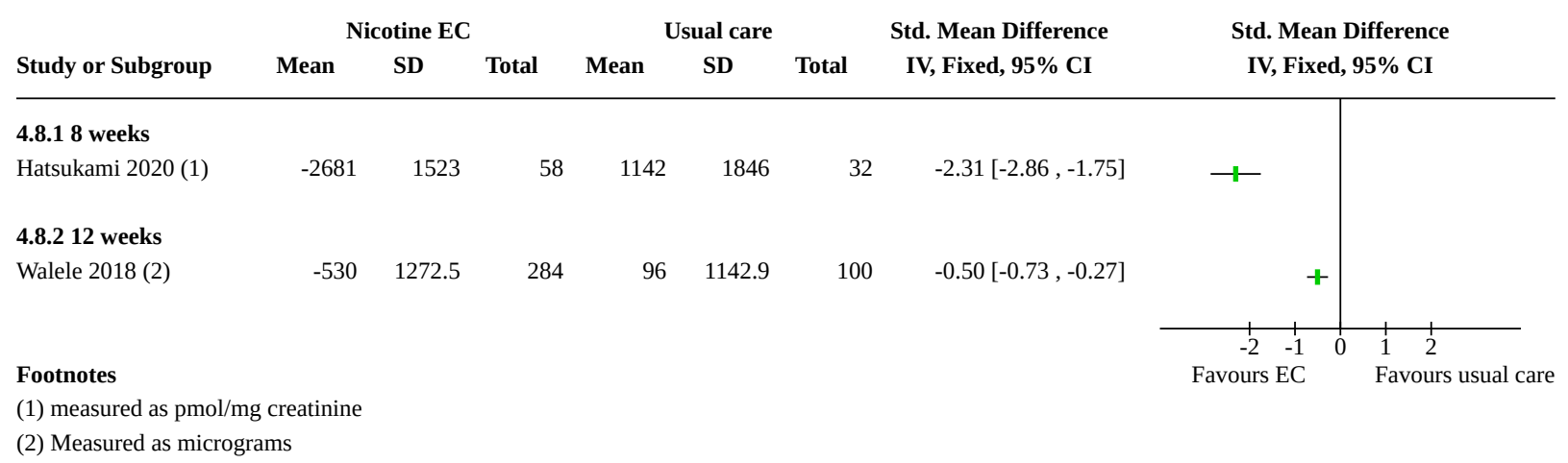

Analysis 4.9. Comparison 4: Nicotine EC versus behavioural support only/no support, Outcome 9: NNAL (SMD)

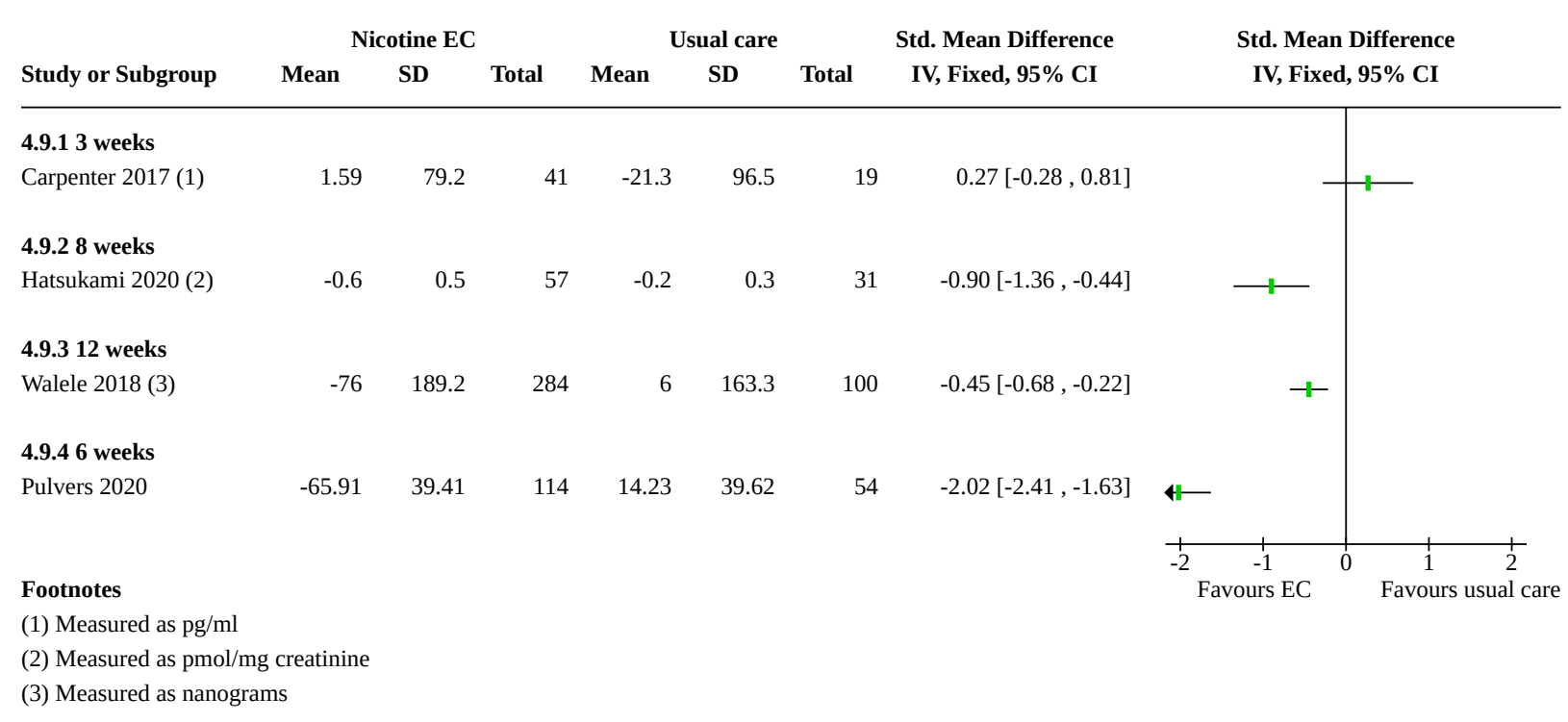


Analysis 4.10. Comparison 4: Nicotine EC versus behavioural support only/no support, Outcome 10: 2-HPMA (pmol/mg creatinine)

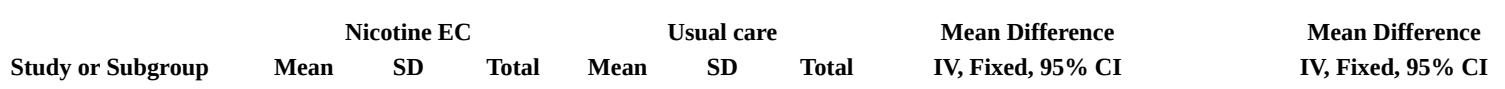

\begin{tabular}{|c|c|c|c|c|c|c|c|}
\hline 4.10.1 8 weeks & & & & & & & \\
\hline Hatsukami 2020 & -249 & 525 & 58 & 264 & 1424 & 32 & $-513.00[-1024.55,-1.45]$ \\
\hline
\end{tabular}

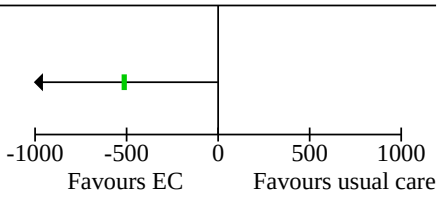

Analysis 4.11. Comparison 4: Nicotine EC versus behavioural support only/no support, Outcome 11: HMPMA (pmol/mg creatinine)

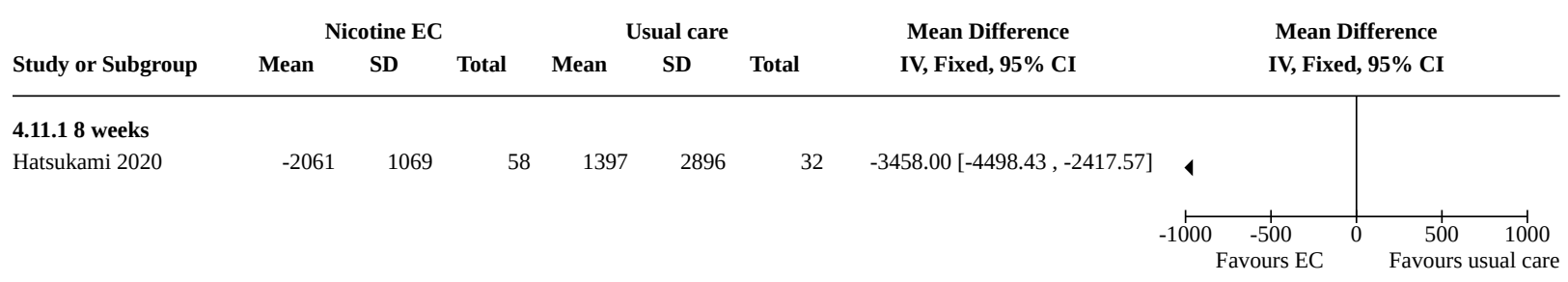

Analysis 4.12. Comparison 4: Nicotine EC versus behavioural support only/no support, Outcome 12: PheT (pmol/mg creatinine)

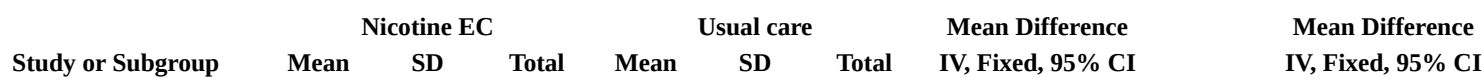

\begin{tabular}{|c|c|c|c|c|c|c|c|}
\hline 4.12.1 8 weeks & & & & & & & \\
\hline Hatsukami 2020 & -0.56 & 0.72 & 56 & 2.2 & 6.72 & 32 & $-2.76[-5.10,-0.42]$ \\
\hline
\end{tabular}

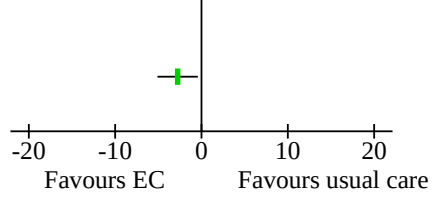

Analysis 4.13. Comparison 4: Nicotine EC versus behavioural support only/no support, Outcome 13: CEMA (pmol/mg creatinine)

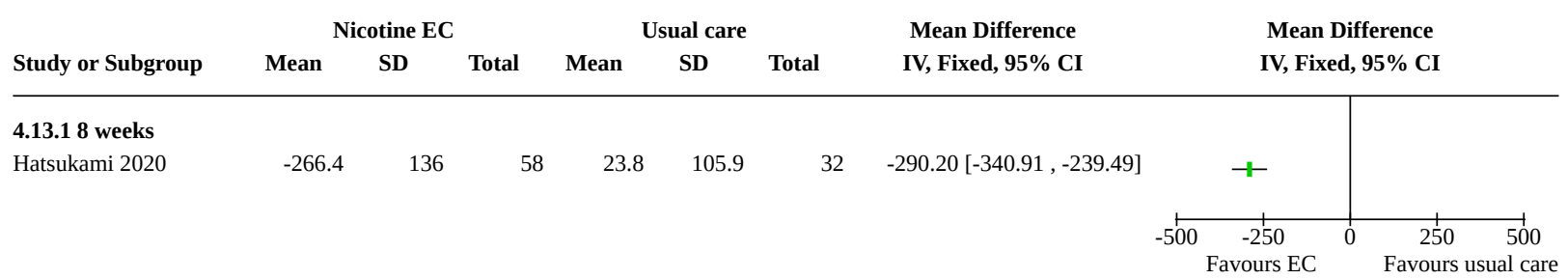


Analysis 4.14. Comparison 4: Nicotine EC versus behavioural support only/no support, Outcome 14: AAMA (pmol/mg creatinine)

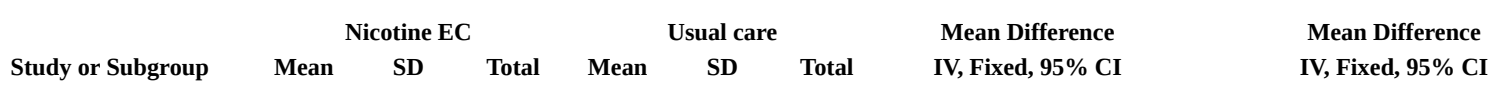

\begin{tabular}{|c|c|c|c|c|c|c|c|}
\hline 4.14.1 8 weeks & & & & & & & \\
\hline Hatsukami 2020 & -144 & 114 & 58 & -4.4 & 127 & 32 & $-139.60[-192.49,-86.71]$ \\
\hline
\end{tabular}

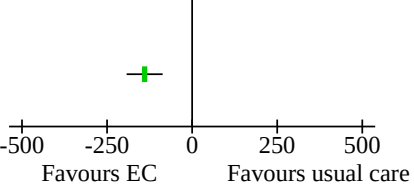

Analysis 4.15. Comparison 4: Nicotine EC versus behavioural support only/no support, Outcome 15: S-PMA (nanograms)

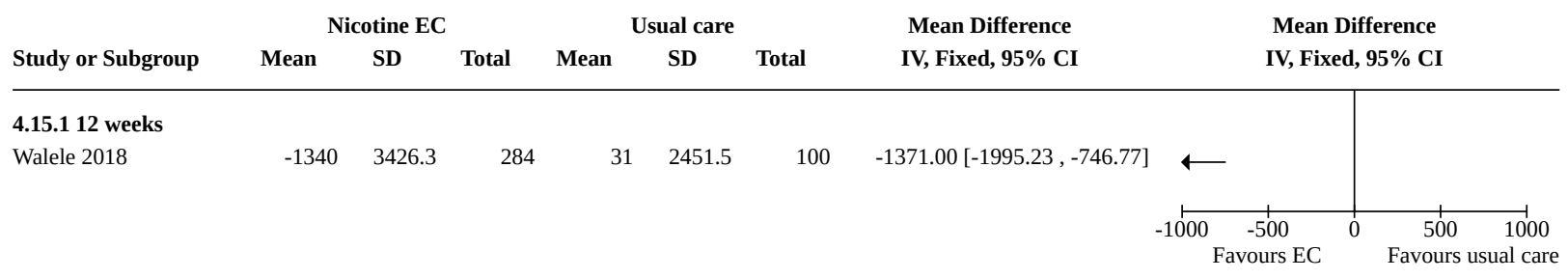

Analysis 4.16. Comparison 4: Nicotine EC versus behavioural support only/no support, Outcome 16: FVC (litres)

\begin{tabular}{lcccccccc} 
& \multicolumn{3}{c}{ Nicotine EC } & \multicolumn{2}{c}{ Usual care } & & Mean Difference & Mean Difference \\
Study or Subgroup & Mean & SD & Total & Mean & SD & Total & IV, Fixed, 95\% CI & IV, Fixed, 95\% CI
\end{tabular}

\begin{tabular}{|c|c|c|c|c|c|c|c|}
\hline 4.16.1 12 we & & & & & & & \\
\hline Walele 2018 & -0.1 & 0.4 & 286 & -0.2 & 0.3 & 101 & $0.10[0.03,0.17]$ \\
\hline
\end{tabular}

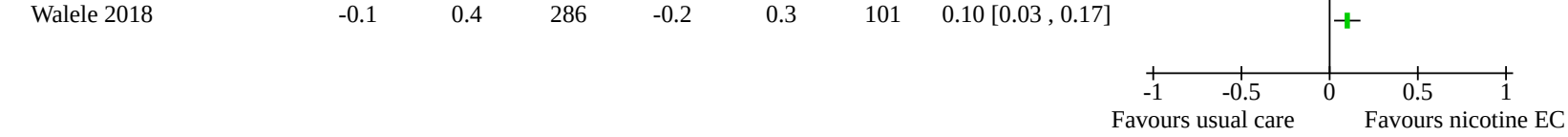

Analysis 4.17. Comparison 4: Nicotine EC versus behavioural support only/no support, Outcome 17: FEV1 (litres)

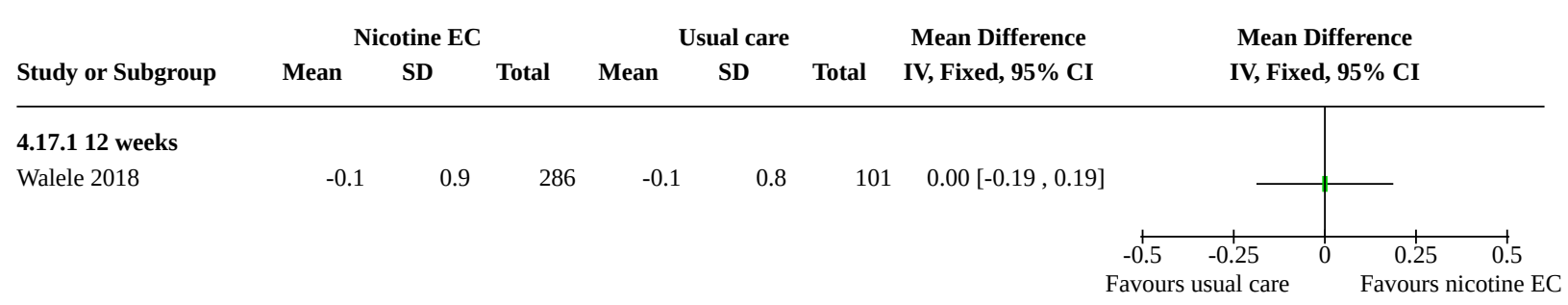




\section{Analysis 4.18. Comparison 4: Nicotine EC versus behavioural support only/no support, Outcome 18: FEF 25-75 (litres/second))}

\begin{tabular}{|c|c|c|c|c|c|c|c|c|c|}
\hline & \multicolumn{3}{|c|}{ Nicotine EC } & \multicolumn{3}{|c|}{ Usual care } & \multicolumn{2}{|r|}{ Mean Difference } & Mean Difference \\
\hline Study or Subgroup & Mean & SD & Total & Mean & SD & Total & Weight & IV, Fixed, 95\% CI & IV, Fixed, 95\% CI \\
\hline
\end{tabular}

\begin{tabular}{lllllllll}
\hline 4.18.1 6 weeks & & & & & & & & \\
Pulvers 2020 & -0.11 & 0.43 & 114 & 0.03 & 0.44 & 54 & $66.8 \%$ & $-0.14[-0.28,0.00]$ \\
Subtotal (95\% CI) & & & $\mathbf{1 1 4}$ & & & $\mathbf{5 4}$ & $\mathbf{6 6 . 8 \%}$ & $\mathbf{- 0 . 1 4}[-\mathbf{0 . 2 8 , 0 . 0 0 ]}$
\end{tabular}

Heterogeneity: Not applicable

Test for overall effect: $\mathrm{Z}=1.94(\mathrm{P}=0.05)$

\subsubsection{2 weeks}

Walele 2018

Subtotal (95\% CI)

$-0.1 \quad 0.4$

286
286

$-0.2 \quad 1$

$101 \quad 33.2 \%$

$0.10[-0.10,0.30]$

Heterogeneity: Not applicable

Test for overall effect: $\mathrm{Z}=0.98(\mathrm{P}=0.33)$

Total (95\% CI)

400

Heterogeneity: $\mathrm{Chi}^{2}=3.68, \mathrm{df}=1(\mathrm{P}=0.06) ; \mathrm{I}^{2}=73 \%$

Test for overall effect: $\mathrm{Z}=1.02(\mathrm{P}=0.31)$

Test for subgroup differences: $\mathrm{Chi}^{2}=3.68, \mathrm{df}=1(\mathrm{P}=0.06), \mathrm{I}^{2}=72.8 \%$

$155 \quad 100.0 \% \quad-0.06[-0.18,0.06]$

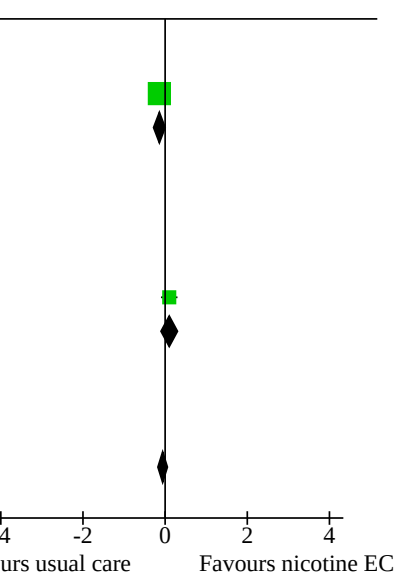

Analysis 4.19. Comparison 4: Nicotine EC versus behavioural support only/no support, Outcome 19: PEF 25-75 (litres/minute)

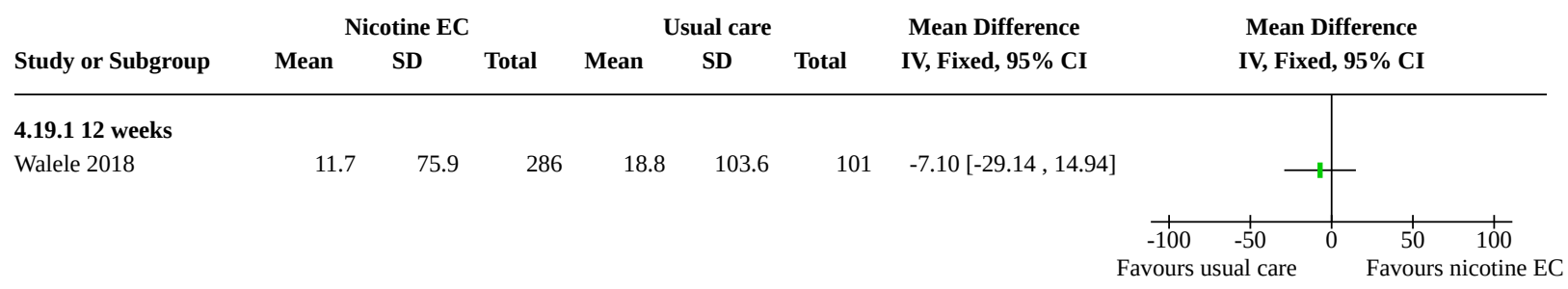

Comparison 5. Nicotine EC + NRT versus non-nicotine EC + NRT

\begin{tabular}{|c|c|c|c|c|}
\hline $\begin{array}{l}\text { Outcome or sub- } \\
\text { group title }\end{array}$ & No. of studies & $\begin{array}{l}\text { No. of partici- } \\
\text { pants }\end{array}$ & Statistical method & Effect size \\
\hline 5.1 Smoking cessation & 2 & 1039 & Risk Ratio (M-H, Fixed, 95\% Cl) & $1.77[1.07,2.94]$ \\
\hline 5.2 Adverse events & 1 & & Risk Ratio (M-H, Fixed, 95\% Cl) & Totals not selected \\
\hline 5.2.1 12 weeks & 1 & & Risk Ratio (M-H, Fixed, 95\% Cl) & Totals not selected \\
\hline $\begin{array}{l}5.3 \text { Serious adverse } \\
\text { events }\end{array}$ & 1 & & Risk Ratio (M-H, Fixed, 95\% Cl) & Totals not selected \\
\hline 5.3.16 months & 1 & & Risk Ratio (M-H, Fixed, 95\% Cl) & Totals not selected \\
\hline $\begin{array}{l}5.4 \text { Carbon monoxide } \\
\text { (ppm) }\end{array}$ & 1 & & Mean Difference (IV, Fixed, 95\% CI) & Totals not selected \\
\hline 5.4.18 weeks & 1 & & Mean Difference (IV, Fixed, 95\% CI) & Totals not selected \\
\hline 5.5 FeNO (ppb) & 1 & 30 & Mean Difference (IV, Fixed, 95\% CI) & $-0.36[-7.23,6.51]$ \\
\hline
\end{tabular}




\begin{tabular}{lllll}
\hline $\begin{array}{l}\text { Outcome or sub- } \\
\text { group title }\end{array}$ & No. of studies & $\begin{array}{l}\text { No. of partici- } \\
\text { pants }\end{array}$ & Statistical method & Effect size \\
\hline 5.5 .16 months & 1 & 30 & Mean Difference (IV, Fixed, 95\% Cl) & $-0.36[-7.23,6.51]$ \\
\hline 5.6 FEV1 (\%) & 1 & 32 & Mean Difference (IV, Fixed, 95\% Cl) & $0.05[-0.01,0.10]$ \\
\hline 5.6 .16 months & 1 & 32 & Mean Difference (IV, Fixed, 95\% Cl) & $0.05[-0.01,0.10]$ \\
\hline 5.7 FVC (\%) & 1 & 32 & Mean Difference (IV, Fixed, 95\% Cl) & $0.03[-0.03,0.09]$ \\
\hline 5.7 .16 months & 1 & 32 & Mean Difference (IV, Fixed, 95\% Cl) & $0.03[-0.03,0.09]$ \\
\hline
\end{tabular}

\section{Analysis 5.1. Comparison 5: Nicotine EC + NRT versus non-nicotine EC + NRT, Outcome 1: Smoking cessation}

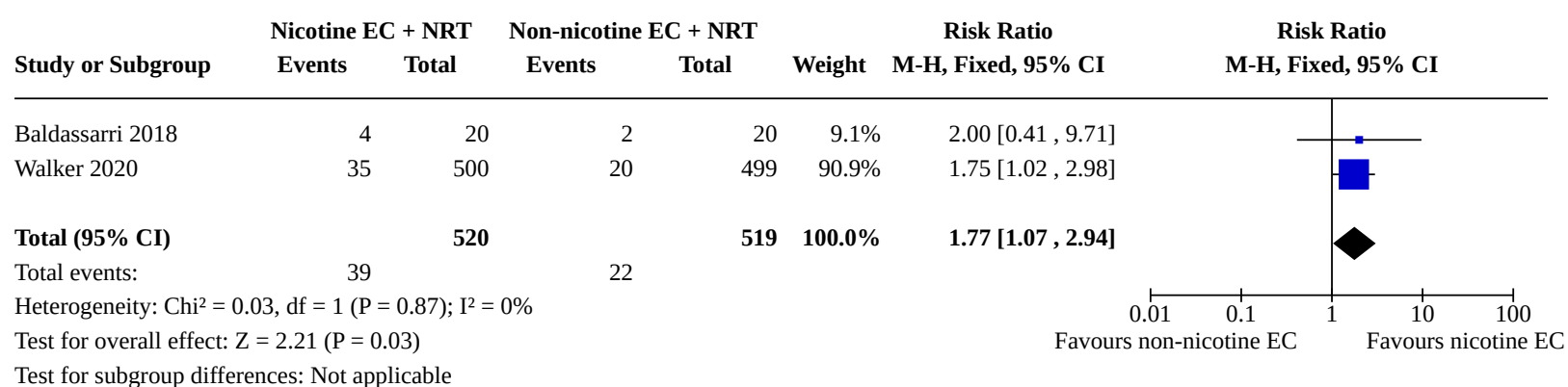

\section{Analysis 5.2. Comparison 5: Nicotine EC + NRT versus non-nicotine EC + NRT, Outcome 2: Adverse events}

\begin{tabular}{ccccccc} 
& \multicolumn{2}{c}{ Nicotine EC + NRT } & \multicolumn{2}{c}{ Non-nicotine EC + NRT } & Risk Ratio & Risk Ratio \\
Study or Subgroup & Events & Total & Events & Total & M-H, Fixed, 95\% CI & M-H, Fixed, 95\% CI
\end{tabular}

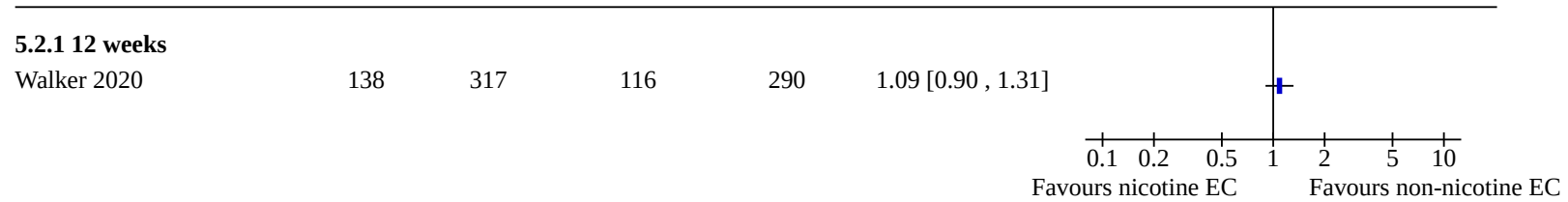

\section{Analysis 5.3. Comparison 5: Nicotine EC + NRT versus non-nicotine EC + NRT, Outcome 3: Serious adverse events}

\begin{tabular}{|c|c|c|c|c|c|}
\hline & Nicoting & + NRT & Non-nicotine EC + NRT & Risk Ratio & Risk Ratio \\
\hline Study or Subgroup & Events & Total & Events & M-H, Fixed, 95\% CI & M-H, Fixed, 95\% CI \\
\hline
\end{tabular}

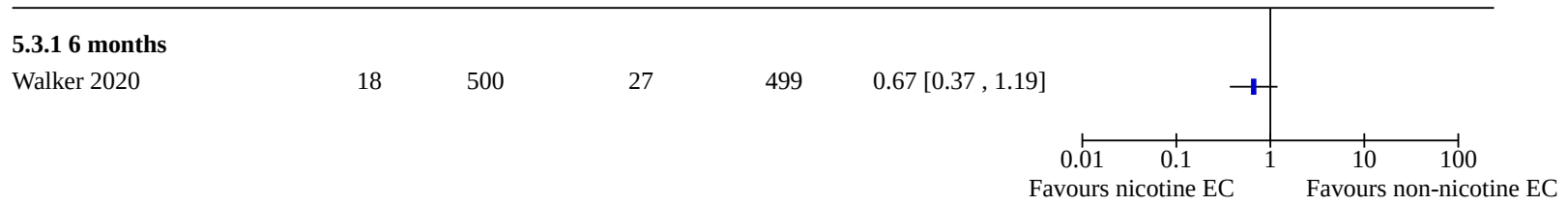


Analysis 5.4. Comparison 5: Nicotine EC + NRT versus non-nicotine EC + NRT, Outcome 4: Carbon monoxide (ppm)

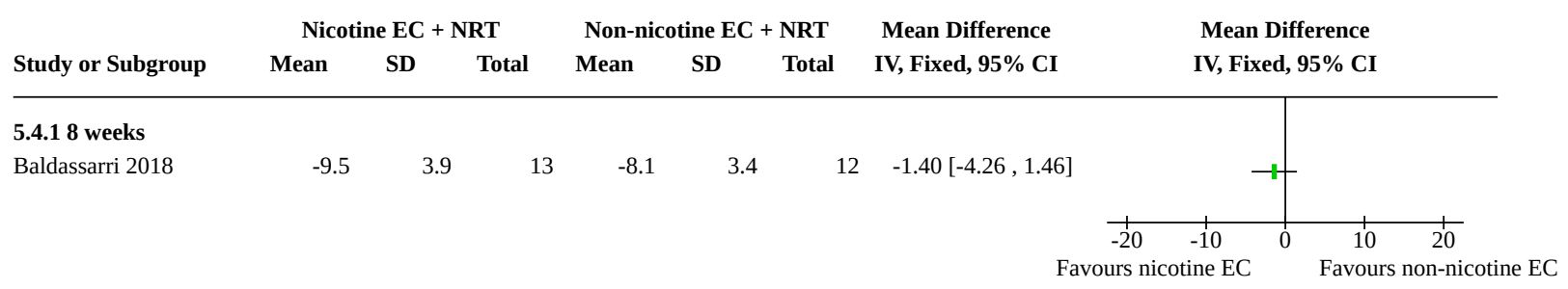

Analysis 5.5. Comparison 5: Nicotine EC + NRT versus non-nicotine EC + NRT, Outcome 5: FeNO (ppb)

\begin{tabular}{lccccccccc}
\multicolumn{4}{c}{ Nicotine EC } & \multicolumn{2}{c}{ Non-nicotine EC } & \multicolumn{2}{c}{ Mean Difference } & Mean Difference \\
Study or Subgroup & Mean & SD & Total & Mean & SD & Total & Weight & IV, Fixed, 95\% CI & IV, Fixed, 95\% CI
\end{tabular}

\subsubsection{6 months}

Baldassarri 2018

(20)

Subtotal (95\% CI)

$2.75 \quad 10.5$

$12 \quad 3.11 \quad 7.45$

$18 \quad 100.0 \%$

IV, Fixed, 95\% CI

Heterogeneity: Not applicable

$18 \quad \mathbf{1 0 0 . 0} \%-0.36[-7.23,6.51]$

Test for overall effect: $\mathrm{Z}=0.10(\mathrm{P}=0.92)$

Total (95\% CI)

12

$18 \quad 100.0 \% \quad-0.36[-7.23,6.51]$

Heterogeneity: Not applicable

Test for overall effect: $\mathrm{Z}=0.10(\mathrm{P}=0.92)$

Test for subgroup differences: Not applicable

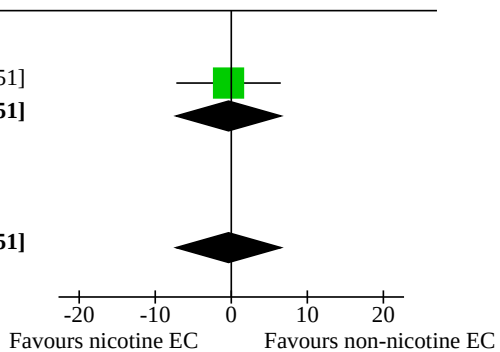

Analysis 5.6. Comparison 5: Nicotine EC + NRT versus non-nicotine EC + NRT, Outcome 6: FEV1 (\%)

\begin{tabular}{lccccccccc} 
& \multicolumn{3}{c}{ Nicotine EC } & \multicolumn{2}{c}{ Non-nicotine EC } & \multicolumn{2}{c}{ Mean Difference } & Mean Difference \\
Study or Subgroup & Mean & SD & Total & Mean & SD & Total & Weight & IV, Fixed, 95\% CI & IV, Fixed, 95\% CI
\end{tabular}

5.6.1 6 months

Baldassarri 2018

$0.0085 \quad 0.057$

$\begin{array}{lll}13 & -0.037 & 0.097\end{array}$

$19 \quad 100.0 \%$

$0.05[-0.01,0.10]$

Subtotal (95\% CI)

$19 \quad \mathbf{1 0 0 . 0} \%$

$0.05[-0.01,0.10]$

Test for overall effect: $\mathrm{Z}=1.67(\mathrm{P}=0.10)$

Total (95\% CI)

13

$19 \quad 100.0 \%$

$0.05[-0.01,0.10]$

Heterogeneity: Not applicable

Test for overall effect: $\mathrm{Z}=1.67(\mathrm{P}=0.10)$

Test for subgroup differences: Not applicable

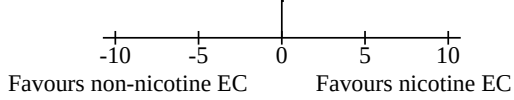

Analysis 5.7. Comparison 5: Nicotine EC + NRT versus non-nicotine EC + NRT, Outcome 7: FVC (\%)

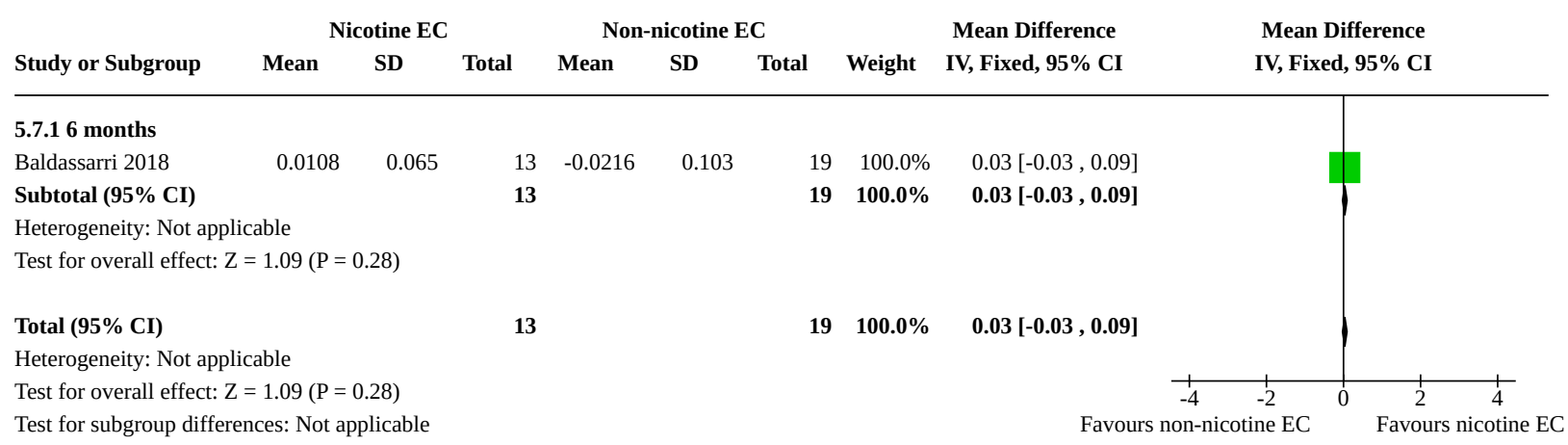


Comparison 6. Nicotine EC + NRT versus NRT

\begin{tabular}{|c|c|c|c|c|}
\hline $\begin{array}{l}\text { Outcome or subgroup } \\
\text { title }\end{array}$ & No. of studies & $\begin{array}{l}\text { No. of partici- } \\
\text { pants }\end{array}$ & Statistical method & Effect size \\
\hline 6.1 Smoking cessation & 1 & & Risk Ratio (M-H, Fixed, 95\% Cl) & Totals not selected \\
\hline 6.2 Adverse events & 2 & & Risk Ratio (M-H, Fixed, 95\% Cl) & Totals not selected \\
\hline 6.2 .112 weeks & 2 & & Risk Ratio (M-H, Fixed, 95\% Cl) & Totals not selected \\
\hline $\begin{array}{l}6.3 \text { Serious adverse } \\
\text { events }\end{array}$ & 3 & 682 & Risk Ratio (M-H, Fixed, 95\% Cl) & $1.26[0.46,3.42]$ \\
\hline 6.3.15 weeks & 1 & 7 & Risk Ratio (M-H, Fixed, 95\% Cl) & Not estimable \\
\hline 6.3 .212 weeks & 1 & 50 & Risk Ratio (M-H, Fixed, 95\% Cl) & $3.00[0.13,70.30]$ \\
\hline 6.3.3 6 months & 1 & 625 & Risk Ratio (M-H, Fixed, 95\% Cl) & $1.12[0.39,3.27]$ \\
\hline
\end{tabular}

\section{Analysis 6.1. Comparison 6: Nicotine EC + NRT versus NRT, Outcome 1: Smoking cessation}

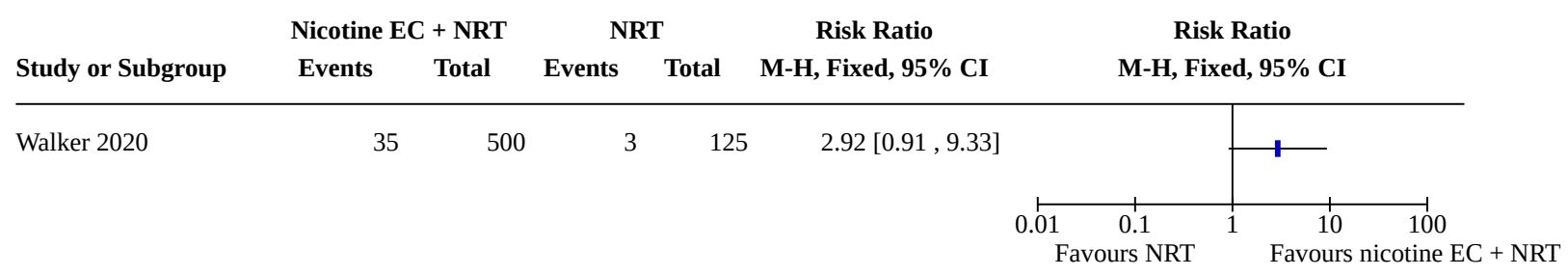

Analysis 6.2. Comparison 6: Nicotine EC + NRT versus NRT, Outcome 2: Adverse events

\begin{tabular}{|c|c|c|c|c|c|c|}
\hline & Nicotine & + NRT & & & Risk Ratio & Risk Ratio \\
\hline Study or Subgroup & Events & Total & Events & Total & M-H, Fixed, 95\% CI & M-H, Fixed, 95\% CI \\
\hline
\end{tabular}

\begin{tabular}{lrrrrr}
\hline 6.2.1 12 weeks & & & & & \\
Guillaumier 2018 (1) & 15 & 25 & 10 & 25 & $1.50[0.84,2.67]$ \\
Walker 2020 & 138 & 317 & 31 & 54 & $0.76[0.58,0.99]$
\end{tabular}

Footnotes

(1) NRT not matched between arms

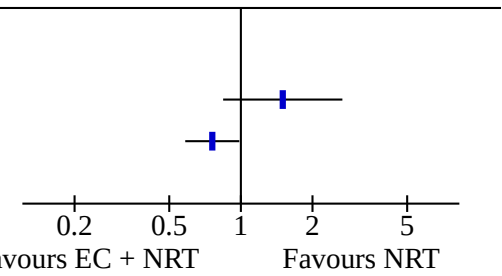

Favours EC + NRT Favours NRT 


\section{Analysis 6.3. Comparison 6: Nicotine EC + NRT versus NRT, Outcome 3: Serious adverse events}

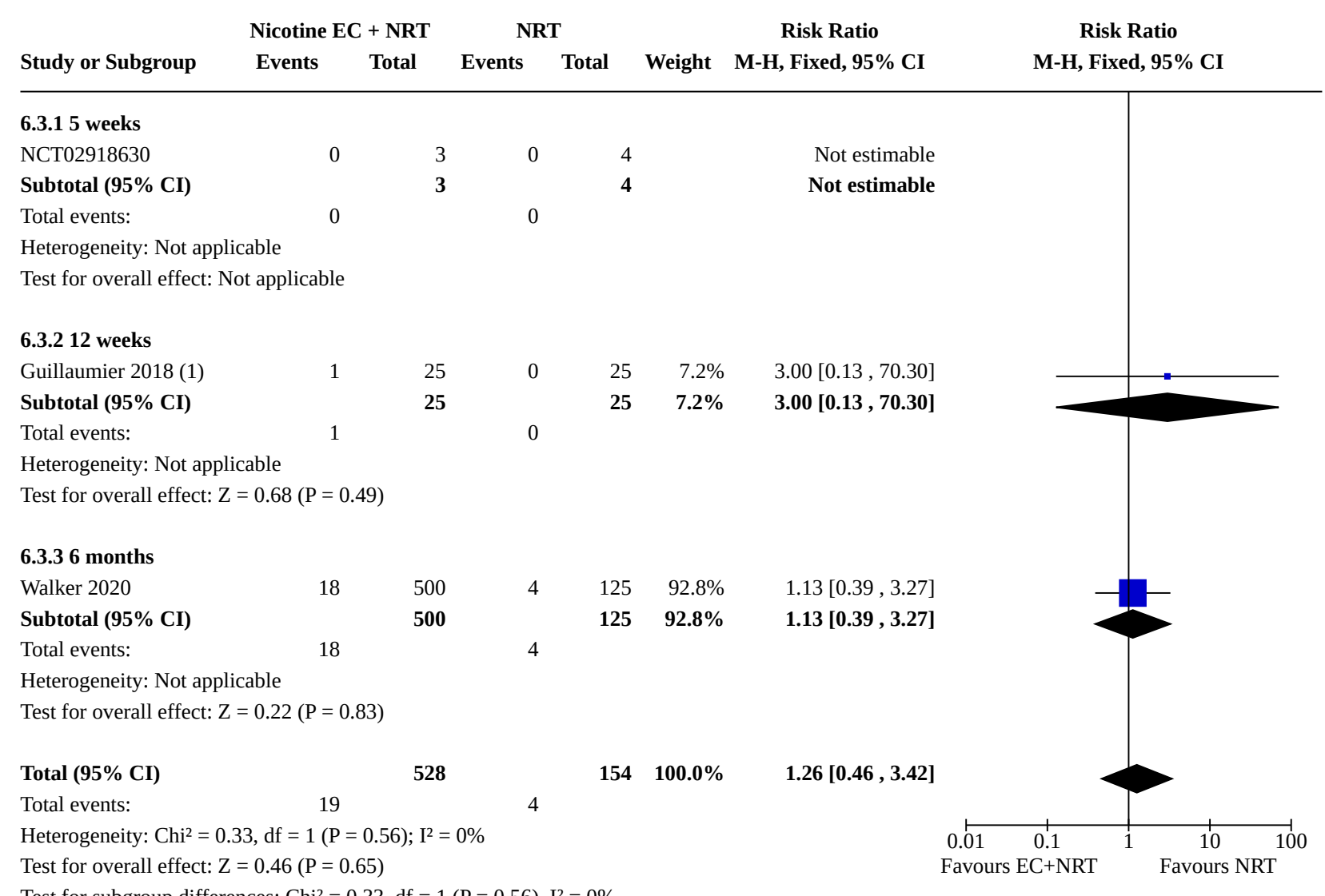

(1) NRT not matched between arms

\section{Comparison 7. Higher versus lower nicotine content}

\begin{tabular}{lllll}
\hline $\begin{array}{l}\text { Outcome or sub- } \\
\text { group title }\end{array}$ & No. of studies & $\begin{array}{l}\text { No. of partici- } \\
\text { pants }\end{array}$ & Statistical method & Effect size \\
\hline $\begin{array}{l}7.1 \text { Serious adverse } \\
\text { events }\end{array}$ & 1 & 72 & Risk Ratio (M-H, Fixed, 95\% Cl) & Not estimable \\
\hline 7.1 .11 year & 1 & 72 & Risk Ratio (M-H, Fixed, 95\% Cl) & Not estimable \\
\hline $\begin{array}{l}7.2 \text { Carbon monoxide } \\
\text { ppm) }\end{array}$ & 1 & Mean Difference (IV, Fixed, 95\% Cl) & Totals not selected \\
\hline 7.2 .112 weeks & 1 & Mean Difference (IV, Fixed, 95\% Cl) & Totals not selected \\
\hline $\begin{array}{l}7.3 \text { Heart rate } \\
7.3 .112 \text { weeks }\end{array}$ & 1 & Mean Difference (IV, Fixed, 95\% Cl) & Totals not selected \\
\hline $\begin{array}{l}7.4 \text { Systolic blood } \\
\text { pressure }\end{array}$ & 1 & Mean Difference (IV, Fixed, 95\% Cl) & Totals not selected \\
\hline
\end{tabular}




\begin{tabular}{|c|c|c|c|c|}
\hline $\begin{array}{l}\text { Outcome or sub- } \\
\text { group title }\end{array}$ & No. of studies & $\begin{array}{l}\text { No. of partici- } \\
\text { pants }\end{array}$ & Statistical method & Effect size \\
\hline 7.4.1 12 weeks & 1 & & Mean Difference (IV, Fixed, 95\% CI) & Totals not selected \\
\hline $7.5 \mathrm{FeNO}(\mathrm{ppb})$ & 1 & & Mean Difference (IV, Fixed, 95\% CI) & Totals not selected \\
\hline 7.5.1 12 weeks & 1 & & Mean Difference (IV, Fixed, 95\% CI) & Totals not selected \\
\hline 7.6 FEV1 (l) & 1 & & Mean Difference (IV, Fixed, 95\% CI) & Totals not selected \\
\hline 7.6.1 12 weeks & 1 & & Mean Difference (IV, Fixed, 95\% CI) & Totals not selected \\
\hline 7.7 FVC (l) & 1 & & Mean Difference (IV, Fixed, 95\% CI) & Totals not selected \\
\hline 7.7.1 12 weeks & 1 & & Mean Difference (IV, Fixed, 95\% CI) & Totals not selected \\
\hline 7.8 FEV1/FVC & 1 & & Mean Difference (IV, Fixed, 95\% CI) & Totals not selected \\
\hline 7.8.1 12 weeks & 1 & & Mean Difference (IV, Fixed, 95\% Cl) & Totals not selected \\
\hline
\end{tabular}

\section{Analysis 7.1. Comparison 7: Higher versus lower nicotine content, Outcome 1: Serious adverse events}

\begin{tabular}{|c|c|c|c|c|c|c|c|}
\hline \multirow[b]{2}{*}{ Study or Subgroup } & \multicolumn{2}{|c|}{ Higher nicotine content } & \multicolumn{2}{|c|}{ Lower nicotine content } & \multicolumn{2}{|r|}{ Risk Ratio } & \multirow{2}{*}{$\begin{array}{c}\text { Risk Ratio } \\
\text { M-H, Fixed, 95\% CI }\end{array}$} \\
\hline & Events & Total & Events & Total & Weight & M-H, Fixed, 95\% CI & \\
\hline
\end{tabular}

\subsubsection{1 year}

Caponnetto 2013a

Subtotal (95\% CI)

Total events:

35

35
35

0

Not estimable

Heterogeneity: Not applicable

Test for overall effect: Not applicable

\section{Total (95\% CI)}

0

37 Not estimable

Total events:

35

Heterogeneity: Not applicable

Test for overall effect: Not applicable

Test for subgroup differences: Not applicable

0

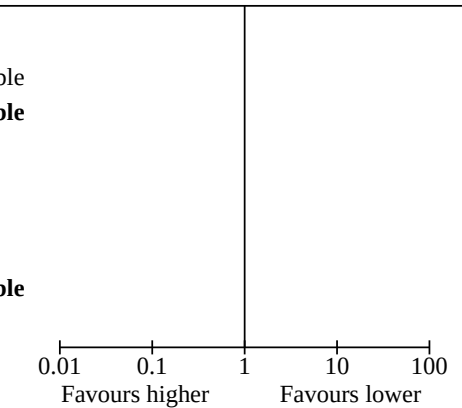

\section{Analysis 7.2. Comparison 7: Higher versus lower nicotine content, Outcome 2: Carbon monoxide (ppm)}

\begin{tabular}{|c|c|c|c|c|c|c|c|c|c|c|}
\hline \multirow[b]{2}{*}{ Study or Subgroup } & \multicolumn{3}{|c|}{ higher dose } & \multicolumn{3}{|c|}{ lower dose } & \multirow{2}{*}{$\begin{array}{c}\text { Mean Difference } \\
\text { IV, Fixed, 95\% CI }\end{array}$} & \multirow{2}{*}{\multicolumn{2}{|c|}{$\begin{array}{l}\text { Mean Difference } \\
\text { IV, Fixed, 95\% CI }\end{array}$}} & \\
\hline & Mean & SD & Total & Mean & SD & Total & & & & \\
\hline \multicolumn{11}{|l|}{ 7.2.1 12 weeks } \\
\hline Caponnetto 2013a & -6 & 6.4 & 76 & -5.8 & 3.4 & 79 & $-0.20[-1.82,1.42]$ & & - & \\
\hline & & & & & & & & $\begin{array}{cc}+10 \\
-20 & -10 \\
\text { ours higher dose }\end{array}$ & $\begin{array}{rr}1 & 1 \\
0 & 10 \\
& \text { Favour }\end{array}$ & $\frac{20}{20 r}$ dose \\
\hline
\end{tabular}


Analysis 7.3. Comparison 7: Higher versus lower nicotine content, Outcome 3: Heart rate

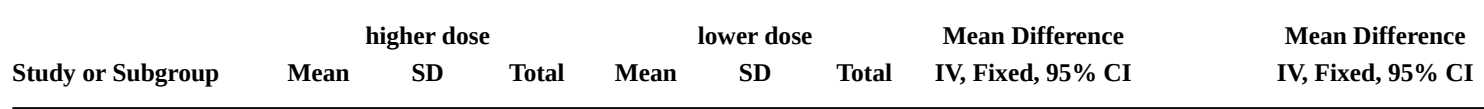

7.3.1 12 weeks

Caponnetto 2013a

$\begin{array}{lllllll}-1.7 & 3.4 & 73 & -1.2 & 3.6 & 75 & -0.50[-1.63,0.63\end{array}$

Analysis 7.4. Comparison 7: Higher versus lower nicotine content, Outcome 4: Systolic blood pressure

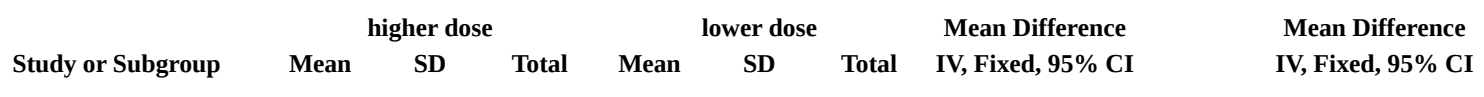

\subsubsection{2 weeks}

Caponnetto 2013a

$\begin{array}{lllllll}-3.9 & 5.7 & 73 & -4.7 & 5.4 & 75 & 0.80[-0.99,2.59]\end{array}$

Analysis 7.5. Comparison 7: Higher versus lower nicotine content, Outcome 5: FeNO (ppb)

\begin{tabular}{|c|c|c|c|c|c|c|c|}
\hline \multirow[b]{2}{*}{ Study or Subgroup } & \multicolumn{3}{|c|}{ higher dose } & \multicolumn{2}{|c|}{ lower dose } & Mean Difference & Mean Difference \\
\hline & Mean & SD & Total & Mean & SD & IV, Fixed, 95\% CI & IV, Fixed, 95\% CI \\
\hline
\end{tabular}

7.5.1 12 weeks

Caponnetto 2013a

$\begin{array}{lllll}2.8 & 1.7 & 49 & 2.5 & 1.6\end{array}$

$0.30[-0.37,0.97]$

Analysis 7.6. Comparison 7: Higher versus lower nicotine content, Outcome 6: FEV1 (I)

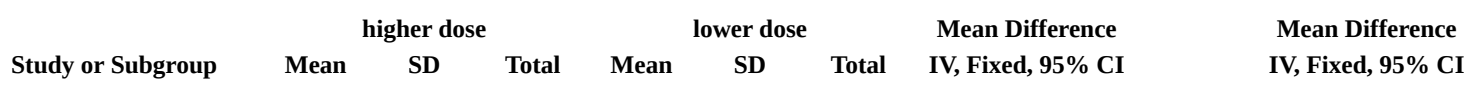

7.6.1 12 weeks

Caponnetto 2013a

$47 \quad 0.01$

0.2

43

$-0.01[-0.11,0.09]$

Analysis 7.7. Comparison 7: Higher versus lower nicotine content, Outcome 7: FVC (I)

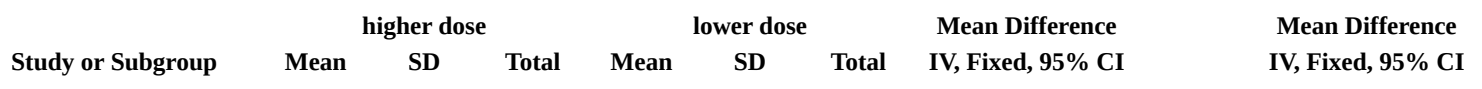

7.7.1 12 weeks

Caponnetto 2013a

$\begin{array}{llll}-0.02 & 0.3 & 47 & 0.01\end{array}$

0.3

$43-0.03[-0.15,0.09]$

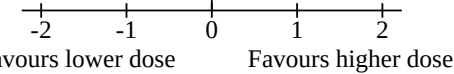

Electronic cigarettes for smoking cessation (Review) 
Analysis 7.8. Comparison 7: Higher versus lower nicotine content, Outcome 8: FEV1/FVC

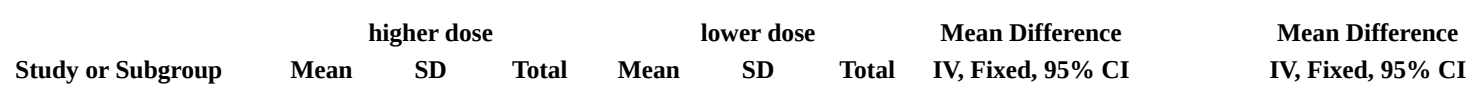

7.8.1 12 weeks

Caponnetto 2013a

$\begin{array}{llll}0.96 & 2 & 47 & 0.05\end{array}$

$1.7 \quad 43$

$0.91[0.15,1.67]$

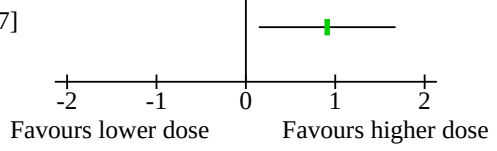

Comparison 8. Non-nicotine EC versus behavioural support only/no support

\begin{tabular}{lllll}
\hline Outcome or subgroup title & No. of studies & $\begin{array}{l}\text { No. of partici- } \\
\text { pants }\end{array}$ & Statistical method & Effect size \\
\hline 8.1 Smoking cessation & 2 & 388 & Risk Ratio (M-H, Fixed, 95\% Cl) & $1.74[0.76,3.96]$ \\
\hline $\begin{array}{l}\text { 8.2 Adverse events at 12 weeks } \\
\text { 8.3 Serious adverse events at 24 }\end{array}$ & 1 & $\begin{array}{l}\text { Risk Ratio (M-H, Random, 95\% } \\
\text { Cl) }\end{array}$ & Totals not selected \\
\hline \begin{tabular}{l} 
weeks \\
\hline
\end{tabular} & & $\begin{array}{l}\text { Risk Ratio (M-H, Random, 95\% } \\
\text { Cl) }\end{array}$ & Totals not selected \\
\hline
\end{tabular}

\section{Analysis 8.1. Comparison 8: Non-nicotine EC versus behavioural support only/no support, Outcome 1: Smoking cessation}

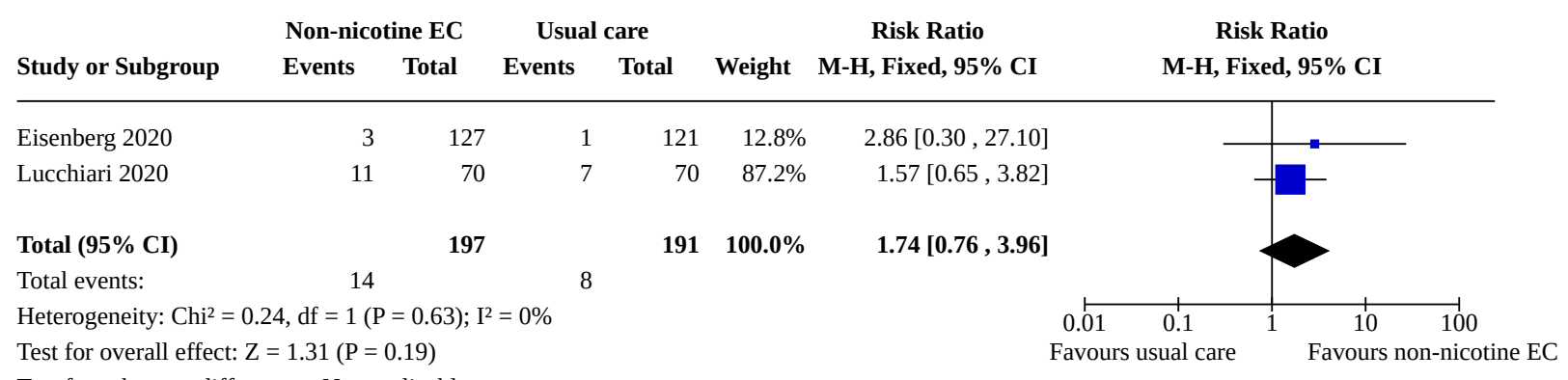

Test for subgroup differences: Not applicable

Analysis 8.2. Comparison 8: Non-nicotine EC versus behavioural support only/no support, Outcome 2: Adverse events at 12 weeks

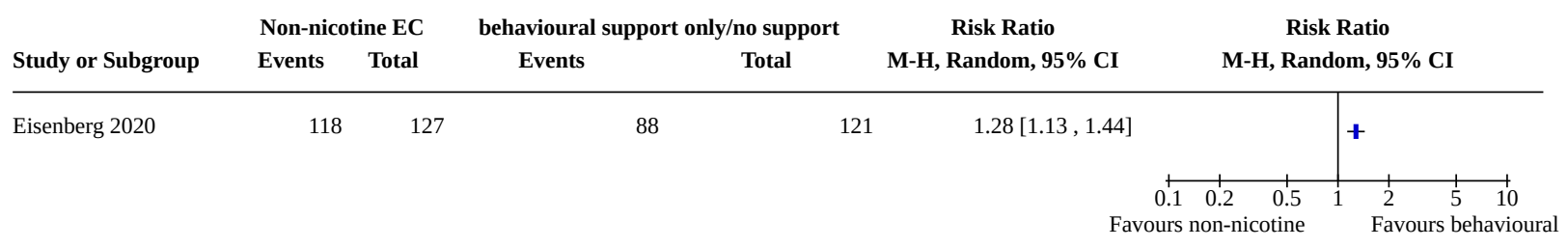


Analysis 8.3. Comparison 8: Non-nicotine EC versus behavioural support only/no support, Outcome 3: Serious adverse events at 24 weeks

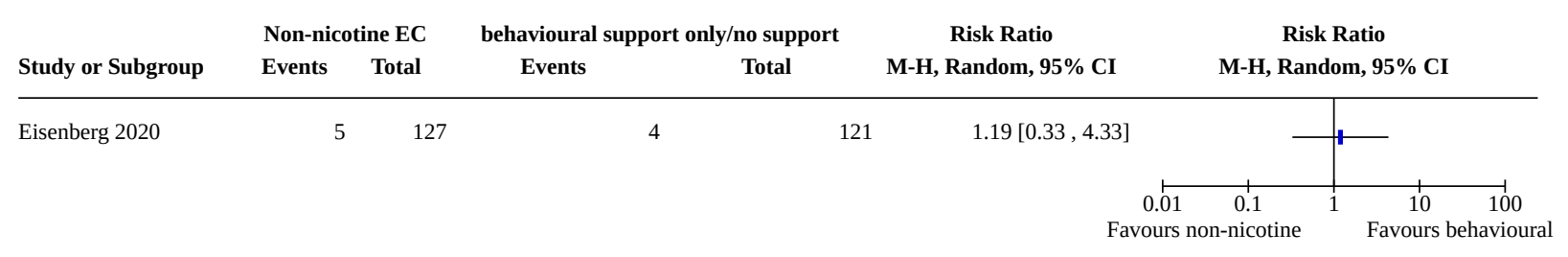

Comparison 9. Non-nicotine EC + NRT versus NRT

\begin{tabular}{lllll}
\hline $\begin{array}{l}\text { Outcome or subgroup ti- } \\
\text { tle }\end{array}$ & No. of studies & $\begin{array}{l}\text { No. of partici- } \\
\text { pants }\end{array}$ & Statistical method & Effect size \\
\hline 9.1 Smoking cessation & 1 & Risk Ratio (M-H, Fixed, 95\% Cl) & Totals not selected \\
\hline 9.2 Adverse events & 1 & Risk Ratio (M-H, Fixed, 95\% Cl) & Totals not selected \\
\hline 9.3 Serious adverse events & 1 & Risk Ratio (M-H, Fixed, 95\% Cl) & Totals not selected \\
\hline 9.3 .16 months & 1 & Risk Ratio (M-H, Fixed, 95\% Cl) & Totals not selected \\
\hline
\end{tabular}

Analysis 9.1. Comparison 9: Non-nicotine EC + NRT versus NRT, Outcome 1: Smoking cessation

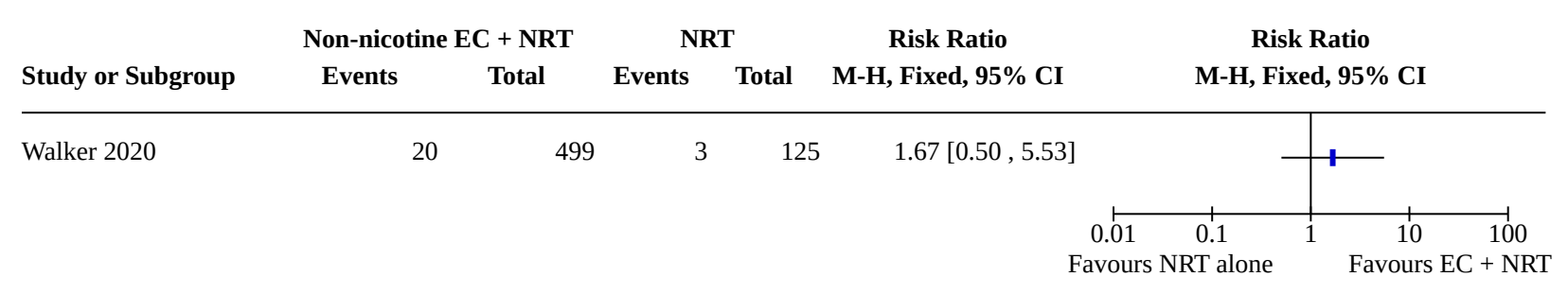

Analysis 9.2. Comparison 9: Non-nicotine EC + NRT versus NRT, Outcome 2: Adverse events

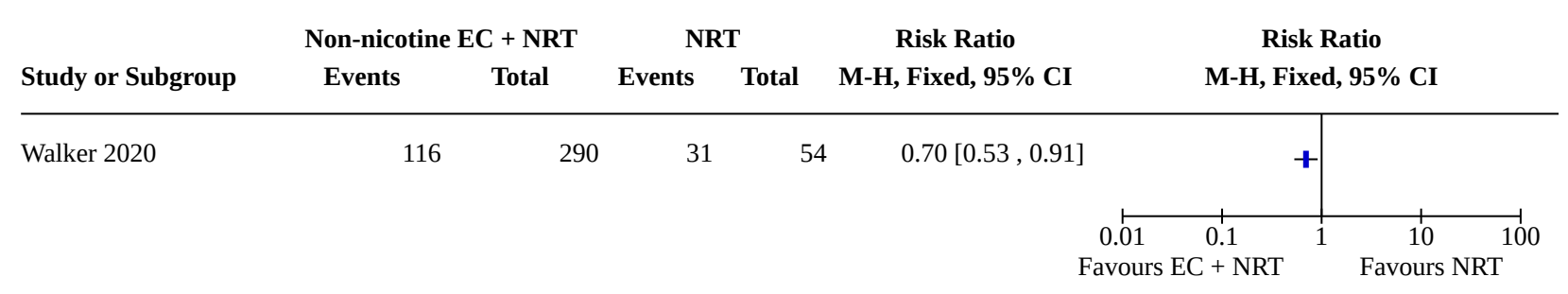


Analysis 9.3. Comparison 9: Non-nicotine EC + NRT versus NRT, Outcome 3: Serious adverse events

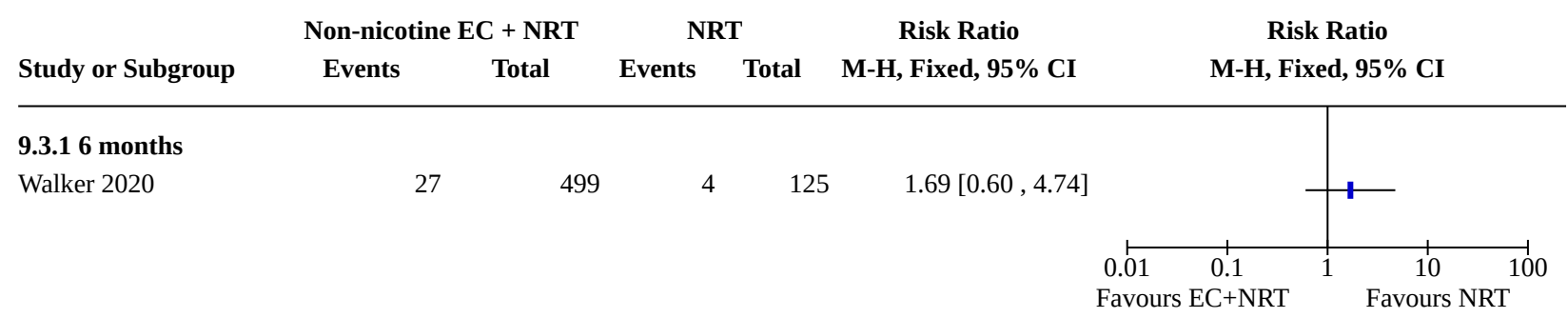

Comparison 10. Non-nicotine EC versus NRT

\begin{tabular}{lllll}
\hline $\begin{array}{l}\text { Outcome or subgroup ti- } \\
\text { tle }\end{array}$ & No. of studies & $\begin{array}{l}\text { No. of partici- } \\
\text { pants }\end{array}$ & Statistical method & Effect size \\
\hline 10.1 Smoking cessation & 1 & & Risk Ratio (M-H, Fixed, 95\% Cl) & Totals not selected \\
\hline 10.2 Adverse events & 1 & Risk Ratio (M-H, Fixed, 95\% Cl) & Totals not selected \\
\hline 10.2 .16 months & 1 & Risk Ratio (M-H, Fixed, 95\% Cl) & Totals not selected \\
\hline $\begin{array}{llll}10.3 \text { Serious adverse } \\
\text { events }\end{array}$ & 1 & Risk Ratio (M-H, Fixed, $95 \% \mathrm{Cl})$ & Not estimable \\
\hline 10.3 .16 months & 1 & 132 & Risk Ratio (M-H, Fixed, 95\% Cl) & Not estimable \\
\hline
\end{tabular}

Analysis 10.1. Comparison 10: Non-nicotine EC versus NRT, Outcome 1: Smoking cessation

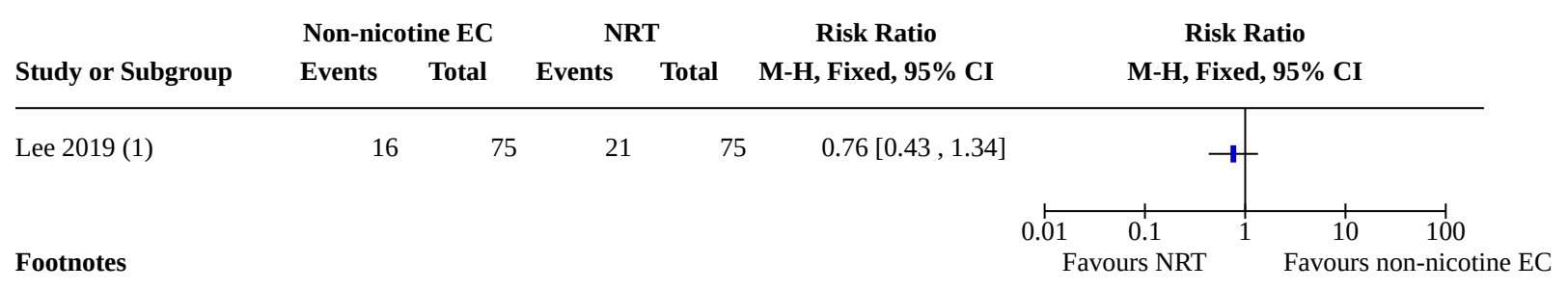

(1) $0.01 \mathrm{mg} / \mathrm{ml}$ of nicotine in e-liquid

\section{Analysis 10.2. Comparison 10: Non-nicotine EC versus NRT, Outcome 2: Adverse events}

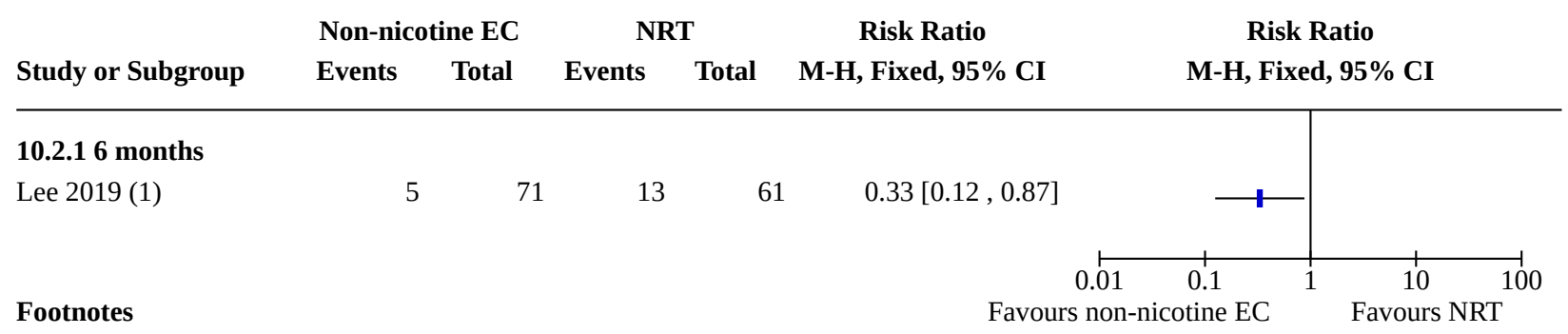

(1) $0.01 \mathrm{mg} / \mathrm{ml}$ of nicotine in e-liquid; length of follow-up not defined but presumably over study period 
Analysis 10.3. Comparison 10: Non-nicotine EC versus NRT, Outcome 3: Serious adverse events

\begin{tabular}{llllllll} 
& \multicolumn{2}{l}{ Non-nicotine EC } & \multicolumn{2}{c}{ NRT } & \multicolumn{2}{c}{ Risk Ratio } & \multicolumn{2}{c}{ Risk Ratio } \\
Study or Subgroup & Events & Total & Events & Total & Weight & M-H, Fixed, 95\% CI & M-H, Fixed, 95\% CI \\
\hline
\end{tabular}

10.3.1 6 months

Lee 2019 (1)

Subtotal (95\% CI)

0

71

0

61

Not estimable

Total events:

$0 \quad 0$

61

Not estimable

Heterogeneity: Not applicable

Test for overall effect: Not applicable

Total (95\% CI)

71

61

Not estimable

Total events:

$0 \quad 0$

Heterogeneity: Not applicable

Test for overall effect: Not applicable

Test for subgroup differences: Not applicable

Footnotes

(1) $0.01 \mathrm{mg} / \mathrm{ml}$ of nicotine in e-liquid; length of follow-up not defined but presumably over study period

\section{ADDITIONAL TABLES}

Table 1. Summary of proportion of participants abstinent from smoking at $6+$ months follow-up: cohort studies of nicotine EC

\begin{tabular}{|c|c|c|c|c|c|c|}
\hline Study & Motivated or unmotivated to & $\%$ abstinent & & & & \\
\hline Cohort studies & & 6-month & 12-month & 18-month & 24-month & Notes \\
\hline Adriaens $2014 a$ & Unmotivated to quit & $19.6 \%(10 / 51)$ & - & - & - & $\begin{array}{l}\text { Data from } \\
\text { 8-month } \\
\text { follow-up }\end{array}$ \\
\hline Bell 2017 & "Willing to attempt to quit" & $26.6 \%(8 / 30)$ & - & - & - & - \\
\hline $\begin{array}{l}\text { Caponnetto } \\
2013 b\end{array}$ & Unmotivated to quit & - & $14 \%(2 / 14)$ & - & - & - \\
\hline Ely 2013 b & Motivated to quit & $44 \%(21 / 48)$ & - & - & - & - \\
\hline Pacifici 2015 & Unmotivated to quit & - & $53 \%(18 / 34)$ & - & - & - \\
\hline Polosa 2011 & Unmotivated to quit & $23 \%(9 / 40)$ & - & $15 \%(6 / 40)$ & $13 \%(5 / 40)$ & - \\
\hline Polosa 2014b & Unmotivated to quit & $36 \%(18 / 50)$ & - & - & - & - \\
\hline Polosa 2015 & Not defined & $42 \%(30 / 71)$ & $41 \%(29 / 71)$ & - & - & - \\
\hline
\end{tabular}

a Technically an RCT but observational for purposes of EC analysis.

bAll participants $(\mathrm{N}=48)$ used an EC, but 16 also used bupropion and 2 used varenicline. 


\section{AP PE N DICES}

\section{Appendix 1. Protocol for living systematic review}

\section{Justification for 'Living Review' status}

Living systematic reviews (LSRs) offer a new approach to updating reviews, in which the review is continually updated by incorporating relevant new evidence as it becomes available (Brooker 2019). Previous versions of this Cochrane Review of electronic cigarettes (ECs) for smoking cessation have informed policy worldwide (Hartmann-Boyce 2016; McRobbie 2014). This update has found high degrees of uncertainty (low- and very low-certainty evidence) for most outcomes, due to the small number of included randomized controlled trials, and the resulting imprecision in effect estimates. This means that some conclusions are likely to change substantially as new evidence emerges.

On average, Cochrane Reviews are updated every three to four years. For EC, where the evidence base is rapidly evolving, this schedule impedes the ability of the review to provide the most up-to-date evidence to decision-makers. As EC use, availability, and design changes, policymakers are frequently drawing on this review to inform decisions, so it is imperative that it is up-to-date to ensure decisions are being made on the basis of the entirety of the evidence. Regular updates have the potential to strengthen the existing conclusions of the review or to change conclusions where conflicting evidence or evidence on new outcomes emerges (e.g. comparisons between EC and other interventions; longer-term safety data).

\section{Objective of the change to 'Living Review' status}

To implement approved Cochrane LSR methods to provide an up-to-date, accessible, engaging and unbiased review of the evidence on the effect and safety of using EC to quit smoking.

\section{LSR methodological considerations}

The methods outlined below are specific to maintaining this review of Electronic cigarettes for smoking cessation as an LSR on the Cochrane Library. These methods will be 'active' immediately upon publication of this update. Core review methods, such as the criteria for considering studies in the review and assessment of risks of bias, are unchanged and are detailed in the main body of the review. Below we outline the methods for which specific considerations apply as a result of the change to 'living' status.

\section{Search methods for identification of studies}

We will conduct database searches monthly, beginning December 2020. These searches will be of the Cochrane Central Register of Controlled Trials (CENTRAL), MEDLINE, Embase, PsycINFO, and clinical trial registries, as detailed in the main body of the review. The funders of this LSR - Cancer Research UK (CRUK) - already run monthly searches of the EC evidence and so we will work alongside their health information officer to ensure that we are identifying all the relevant literature with our searches. We will review our search strategies on an ongoing basis every 12 months, as indexing terms and keywords may change, and new search filters may be published. Such changes will be managed by input from experienced information specialists.

\section{Selection of studies}

We will immediately screen any new citations retrieved by the monthly searches using Covidence, undertaking dual screening of title and abstract, and then full text, by independent review authors. Where we find multiple citations of the same study we will group them into one study record with a single study ID. One review author $(A B)$ will contact corresponding authors of potentially relevant ongoing studies as they are identified and ask them to advise when results are available, or to share early or unpublished data. Based on the information and projected timescales shared, we will contact corresponding authors on an ongoing basis to retrieve new evidence as it becomes available.

\section{Data synthesis}

Whenever we identify new studies relevant to the review, we will extract the relevant data and assess risks of bias as detailed in the main body of the review. We will highlight availability of this new evidence on both the Cochrane Library and on our own dedicated website. We will incorporate the new data into meta-analyses and tables in the Revman (Review Manager 2020) and supplementary data files, and carry out GRADE assessments (GRADEpro GDT). We will conduct a full update of the review (full incorporation and interpretation of all new data within the review and re-publishing) when the accumulating evidence leads to changes in any one of:

- The direction of effect or clinical significance of the findings for one or more outcomes;

- The certainty (e.g. GRADE rating) of one or more outcomes;

- The availability of studies investigating new settings, populations, interventions, comparisons or outcomes.

Formal sequential meta-analysis approaches will not be used for updated meta-analyses, in line with Cochrane guidance for LSRs.

\section{Future updates of review methods}

The LSR approach acknowledges that reviews may cease to need to be 'living' over time, as the review findings become stable, or the question is no longer a priority for decision-makers (Brooker 2019). Eighteen months into this review's 'living' status (March 2022) we will 
evaluate the LSR approach, including the likely benefits of and challenges to continuing this methodology for this evidence base, and whether such an approach remains warranted. If the evidence is high certainty for all outcomes and all comparisons at that point, meaning further studies are judged very unlikely to impact the effect estimate, we would consider ceasing living mode for this review. If, as is more likely, some or all outcomes are not yet certain, we will facilitate discussions within the author team and Cochrane, as well as engaging with a wider PPI panel and key decision-makers, e.g. policymakers, in order to determine next steps. If the decision is made to continue in living mode, we will review, and if necessary revise, the living review methods described in this Appendix before continuing.

\section{Appendix 2. Toxins/carcinogen names and abbreviations}

\begin{tabular}{|c|c|}
\hline Abbreviation & Name \\
\hline- & 1-Hydroxyfluorene \\
\hline- & 1-Hydroxyphenanthrene \\
\hline- & 1-Hydroxypyrene \\
\hline 2-HPMA & 2-hydroxypropylmercapturic acid \\
\hline- & 2-Hydroxyfluorene \\
\hline- & 2-Hydroxyphenanthrene \\
\hline- & 2-Naphthol \\
\hline- & 3-, 4-Hydroxyphenanthrenes \\
\hline 3-HPMA & 3-hydroxypropylmercapturic acid \\
\hline- & 3-Hydroxyfluorene \\
\hline AAMA & $\mathrm{N}$-acetyl-S-(carbamoylethyl)-L-cysteine (synonym: 2-carbamoylethylmercapturic acid) \\
\hline CEMA/CNEMA & 2-cyanoethylmercapturic acid; referred to as 'acrylonitrile' in Pulvers 2018 \\
\hline- & Formic acid \\
\hline HEMA & 2-hydroxyethylmercapturic acid \\
\hline HMPMA/HPMMA & 3-hydroxy-1-methyl propylmercapturic acid \\
\hline MHBMA & 2-hydroxy-3-buten-1-ylmercapturic acid \\
\hline MMA & $\mathrm{N}$-nitrosodimethyamine \\
\hline NNAL & 4-(methylnitrosamino)-1-(3-pyridyl)-1-butanol \\
\hline PheT & Phenanthrene tetraol \\
\hline PMA & phenylmercapturic acid; referred to as 'benzene' in Pulvers 2018 \\
\hline S-PMA & S-phenylmercapturic acid \\
\hline
\end{tabular}




\section{Appendix 3. MEDLINE search strategy - 2020 update}

1. exp case control studies/ or exp cohort studies/ or Case control.tw. or (cohort adj (study or studies)).tw. or Cohort analy\$.tw. or (Follow up adj (study or studies)).tw. or (observational adj (study or studies)).tw. or Longitudinal.tw.

2. (e-cig\$ or ecig\$ or electr\$ cigar\$ or electronic nicotine).mp. or (vape or vapes or vaporizer or vapourizer or vaporiser or vapouriser or vaper or vapers or vaping).ti,ab. or exp Electronic Nicotine Delivery Systems/

3. (randomized controlled trial or controlled clinical trial).pt. or randomized.ab. or placebo.ab. or clinical trials as topic.sh. or randomly.ab. or trial.ti.

4. exp animals/ not humans.sh.

5. 3 not 4

6. 2 and 5

7. 1 and 2

8.6 or 7

9. smoking cessation.mp. or exp Smoking Cessation/

10. tobacco cessation.mp. or "Tobacco-Use-Cessation"/

11. (nicotine dependence or tobacco dependence).mp.

12. exp Smoking/th

13. "Tobacco-Use-Disorder"/

14. Smoking reduction/ or Smoking reduction.mp.

15. exp Pipe smoking/ or exp Tobacco smoking/ or exp Tobacco Products/

16. ((quit\$ or stop\$ or ceas\$ or giv\$ or abstain* or abstinen*) adj5 (smoking or smoke* or tobacco)).ti,ab.

17. exp Tobacco/ or exp Nicotine/

18.9 or 10 or 11 or 12 or 13 or 14 or 15 or 16 or 17

19. 8 and 18

\section{Appendix 4. MEDLINE search strategy - pre-2020}

1. e-cig\$.mp. [mp=title, abstract, original title, name of substance word, subject heading word, protocol supplementary concept, rare disease supplementary concept, unique identifier]

2. electr\$ cigar\$.mp.

3. electronic nicotine.mp.

4. (vape or vaper or vapers or vaping).ti,ab.

5. 1 OR 2 OR 3 OR 4

Identical terms used for other databases.

Line 4 added to search strategy for 2016 update.

WHAT'S NEW

\begin{tabular}{lll}
\hline Date & Event & Description \\
\hline 15 April 2021 & New search has been performed & $\begin{array}{l}\text { Updated with six new included studies and new data from one } \\
\text { previously included study. Most recent search 1 Feb 2021. }\end{array}$ \\
\hline 15 April 2021 & $\begin{array}{l}\text { New citation required and conclusions } \\
\text { have changed }\end{array}$ & $\begin{array}{l}\text { 6 new included studies added (Czoli 2019; lkonomidis 2020; Oz- } \\
\text { ga-Hess 2019; Pulvers 2020; Scheibein 2020; Yingst 2020), cer- }\end{array}$ \\
\hline
\end{tabular}

Electronic cigarettes for smoking cessation (Review) 


\begin{tabular}{lll}
\hline Date Event Description & Den
\end{tabular}

tainty in finding of no difference in adverse events between nicotine EC and non-nicotine EC updated to moderate (from low). First study of pod EC device included.

\section{H I S T O R Y}

Protocol first published: Issue 11, 2012

Review first published: Issue 12, 2014

\begin{tabular}{lll}
\hline Date & Event & Description \\
\hline 1 April 2021 & Amended & $\begin{array}{l}\text { This is a Living Systematic Review. We run and screen searches } \\
\text { monthly. Last search date 1st April 2021. In addition to the stud- } \\
\text { ies identified from March 2021 we found two new ongoing stud- } \\
\end{array}$ \\
& $\begin{array}{l}\text { ies and one paper linked to a study already included in the re- } \\
\text { view. We will incorporate these into the review as part of a future } \\
\text { update. }\end{array}$
\end{tabular}

17 March $2021 \quad$ Amended

This is a Living Systematic Review. We run and screen searches monthly. Last search date 1st March 2021. Studies identified in March are not included in this version of the review, but will be incorporated into a subsequent version. We found four new included studies, five new ongoing studies and five papers linked to studies already included in the review. The four new included studies were all conference abstracts; three of which were identified from the SRNT 2021 abstract book (SYM2A, SYM2B, PH-353; www.srnt.org/page/2021_Meeting). The fourth is available here: dx.doi.org/10.1016/j.drugalcdep.2015.07.1091.

4 February $2021 \quad$ Amended

This is a Living Systematic Review. We run and screen searches monthly. Last search date 1st February 2021. In addition to the studies identified from our December 2020 and January 2021 searches we found one paper linked to a study already included in the review (Lucchiari 2020), and have preliminary results from a study listed as ongoing (Begh 2019). We will incorporate this paper and data into the review as part of a future update.

20 January $2021 \quad$ Amended

This is a Living Systematic Review. Searches are run and screened monthly. Last search date 4th January 2021. In addition to the studies identified from our December 2020 searches we found four new completed studies, one new ongoing study and one paper linked to a study already included in the review. These studies and papers will be incorporated into the review at the next update. DOls for the four new included studies are as follows: Ozga-Hess et al. 2019: 10.1016/j.addbeh.2019.106105; Pulvers et al. 2020: 10.1001/jamanetworkopen.2020.26324; Scheibein 2020: 10.1186/s12954-020-00406-y; Yingst et al. 2020: 10.1080/09540121.2019.1687835

15 December 2020 Amended
This is a Living Systematic Review. Searches are run and screened monthly. Last search date 1st December 2020. Searches found 3 new completed studies, 11 new ongoing studies and 9 papers linked to studies already included in the review. These studies and papers will be incorporated into the review at the next update. DOIs for the three new included studies are as fol- 


\begin{tabular}{|c|c|c|}
\hline Date & Event & Description \\
\hline & & $\begin{array}{l}\text { lows: Czoli et al:10.1093/ntr/nty174; Bonevski et al: } 10.1093 / \text { ntr/ } \\
\text { ntaa143; Eisenberg et al: } 10.1001 / \text { jama.2020.18889. }\end{array}$ \\
\hline 20 July 2020 & $\begin{array}{l}\text { New citation required and conclusions } \\
\text { have changed }\end{array}$ & $\begin{array}{l}\text { Strength of evidence increased for existing comparisons; new } \\
\text { comparisons added }\end{array}$ \\
\hline 20 July 2020 & New search has been performed & $\begin{array}{l}\text { New searches run January 2020. } 35 \text { new studies added. Living } \\
\text { systematic review protocol incorporated }\end{array}$ \\
\hline 14 December 2016 & Amended & $\begin{array}{l}\text { Clarification on outcome data from Adriaens - no changes to con- } \\
\text { clusions }\end{array}$ \\
\hline 23 June 2016 & $\begin{array}{l}\text { New citation required but conclusions } \\
\text { have not changed }\end{array}$ & 11 new included studies added; no changes to conclusions. \\
\hline
\end{tabular}

\section{CONTRIBUTIONS OF AUTHORS}

All authors contributed to the writing of this review.

For this update, JHB, NL, CN, RB, PH, NR, ARB, HMR screened studies and/or extracted data.

$J H B$ and $A R B$ entered data for analysis.

\section{DECLARATIONS OF INTEREST}

RB holds an NIHR grant, however this did not directly fund this current work. She is principal investigator of an ongoing study listed in this review.

CB was principal investigator on the ASCEND e-cigarette trial reported in the Cochrane review and a co-investigator on the ASCEND II trial and several other studies included in the review. CB has provided consultancy for J\&J KK (Japan) on NRT products. CB reports research grants from the Health Research Council of NZ, the Heart Foundation of NZ and the NZ Ministry of Health. He has recently led a project funded by Pfizer (NZ) on chronic disease management.

ARB's work on this review has been supported by Cancer Research UK Project Award funding. This is not deemed a conflict of interest.

$\mathrm{PH}$ provided consultancy for and received research funding from Pfizer, a manufacturer of stop-smoking medications. He was principal investigator on one of the trials included in this review and co-investigator on other relevant studies.

JHB has received support for this work from the Cochrane Review Support Programme and the University of Oxford's Returning Carer's Fund. Neither of these are deemed conflicts of interest.

NL has received payment for lectures on systematic review methodology, and has been an applicant on project funding to carry out priority setting and systematic reviews in the area of tobacco control (NIHR funded). None of this is deemed a conflict of interest.

HM has received honoraria for speaking at smoking cessation educational events and sitting on an advisory board organized by Pfizer.

CN has no known conflicts of interest.

NR has received royalties from UpToDate, Inc., for chapters on electronic cigarettes and occasional fees from academic hospitals or professional medical societies for lectures on smoking cessation that include discussion of electronic cigarettes. Dr. Rigotti was an member of the committee that produced the 2018 National Academies of Science, Engineering, and Medicine's Consensus Study Report on the Public Health Benefits of E-cigarettes. She was unpaid for this work. Outside the topic of e-cigarettes, Dr. Rigotti has received honoraria from Achieve Life Sciences for consulting about cytisine. NR is a consultant for Achieve LifeSciences, which is developing an investigational smoking cessation medication for FDA approval (cytisine) and her institution (MGH) receives a grant from the company as a site for a clinical trial testing the safety and efficacy of cytisine. NR has received travel reimbursement (but no honoraria) from Pfizer for attending advisory boards regarding varenicline. NR holds grants from NIH for research work. 
AT's work on this review has been supported by the Cochrane Review Support Programme and the University of Oxford's Returning Carer's Fund. Neither of these are deemed conflicts of interest.

TT has no known conflicts of interest.

\section{SOURCES OF SUPPORT}

\section{Internal sources}

- Queen Mary University of London, UK provides salary, office space and library resources for $\mathrm{HM}$ and $\mathrm{PH}$

- The University of Auckland, New Zealand provides salary, office space and library resources for CB

- University of Oxford, UK Support from Returning Carers' Fund

\section{External sources}

- NIHR, UK

Infrastructure award for Cochrane Tobacco Addiction Group and Cochrane Incentive Award

- Cancer Research UK, UK

Cancer Research UK project award funding to support living systematic review

\section{DIFFERENCES BETWEEN PROTOCOLAND REVIEW}

The protocol did not specify a minimum follow-up period for data on adverse events. As of the 2016 update, we have changed the Methods section to clarify that we will exclude follow-up data at less than a week.

The original version of this review included reduction as a secondary outcome. The 2016 update removed reduction as an outcome, to bring the review into line with other reviews of cessation treatments produced by the Cochrane Tobacco Addiction Group and to prevent substantial overlap with the update of the Group's review of interventions for harm reduction.

As prespecified in the 2016 update, in the 2020 update we excluded non-intervention studies. In the 2020 update, we also added in an appendix with a protocol setting out our plans to convert this review into a living systematic review in the future.

\section{INDEX TERMS}

\section{Medical Subject Headings (MeSH)}

Bias; Cohort Studies; *Electronic Nicotine Delivery Systems; *Nicotine [administration \& dosage]; *Nicotinic Agonists [administration \& dosage]; Publication Bias; Randomized Controlled Trials as Topic; Smoking [epidemiology]; Smoking Cessation [ ${ }^{\star}$ methods] [statistics \& numerical data]; *Smoking Prevention; Tobacco Use Cessation Devices; Vaping

\section{MeSH check words}

Humans; Middle Aged 